\title{
A Spectroscopic and Photometric Survey of Novae in M31
}

\author{
A. W. Shafter ${ }^{1}$, M. J. Darnley ${ }^{2}$, K. Hornoch ${ }^{3}$, A. V. Filippenko ${ }^{4}$, M. F. Bode ${ }^{2}$, R. \\ Ciardullo $^{5}$, K. A. Misselt ${ }^{6}$, R. A. Hounsell ${ }^{2}$, R. Chornock $^{4,7}$, and T. Matheson ${ }^{8}$
}

\begin{abstract}
We report the results of a multi-year spectroscopic and photometric survey of novae in M31 that resulted in a total of 53 spectra of 48 individual nova candidates. Two of these, M31N 1995-11e and M31N 2007-11g, were revealed to be long-period Mira variables, not novae. These data double the number of spectra extant for novae in M31 through the end of 2009 and bring to 91 the number of M31 novae with known spectroscopic classifications. We find that 75 novae $(82 \%)$ are confirmed or likely members of the Fe II spectroscopic class, with the remaining 16 novae (18\%) belonging to the He/N (and related) classes. These numbers are consistent with those found for Galactic novae. We find no compelling evidence that spectroscopic class depends sensitively on spatial position or population within M31 (i.e., bulge vs. disk), although the distribution for $\mathrm{He} / \mathrm{N}$ systems appears slightly more extended than that for the Fe II class. We confirm the existence of a correlation between speed class and ejection velocity (based on line width), as in the case of Galactic novae. Follow-up photometry allowed us to determine light-curve parameters for a total of 47 of the 91 novae with known spectroscopic class. We confirm that more luminous novae generally fade the fastest, and that $\mathrm{He} / \mathrm{N}$ novae are typically faster and brighter than their Fe II counterparts. In addition, we find a weak dependence of nova speed class on position in M31, with the spatial distribution of the fastest novae being slightly more extended than that of slower novae.
\end{abstract}

\footnotetext{
${ }^{1}$ Department of Astronomy, San Diego State University, San Diego, CA 92182, USA

${ }^{2}$ Astrophysics Research Institute, Liverpool John Moores University, Birkenhead CH41 1LD, UK

${ }^{3}$ Astronomical Institute, Academy of Sciences, CZ-251 65 Ondřejov, Czech Republic

${ }^{4}$ Department of Astronomy, University of California, Berkeley, CA 94720-3411, USA

${ }^{5}$ Department of Astronomy and Astrophysics, The Pennsylvania State University, 525 Davey Lab, University Park, PA 16802, USA

${ }^{6}$ Steward Observatory, University of Arizona, Tucson, AZ, 85721, USA

${ }^{7}$ Harvard-Smithsonian Center for Astrophysics, 60 Garden Street, Cambridge, MA 02138, USA

${ }^{8}$ National Optical Astronomy Observatory, 950 North Cherry Avenue, Tucson, AZ 85719-4933, USA
} 
Subject headings: galaxies: stellar content — galaxies: individual (M31) — stars: novae, cataclysmic variables

\section{Introduction}

Classical novae form a subclass of the cataclysmic variable stars. They are semidetached binary star systems where a late-type Roche-lobe-filling star transfers mass to a white dwarf companion (e.g., Warner 1995, 2008). If the mass accretion rate onto the white dwarf is sufficiently low to allow the accreted gas to become degenerate, a thermonuclear runaway (TNR) will eventually ensue in the accreted envelope, leading to a nova eruption. These eruptions can reach an absolute magnitude as bright as $\mathrm{M}_{V} \approx-10$ (e.g., Starrfield et al. 2008), making them among the most luminous explosions in the Universe. Their high lumi-

nosities and rates ( $\sim 50 \mathrm{yr}^{-1}$ in a galaxy like M31; Shafter \& Irby 2001; Darnley et al. 2006) make novae powerful probes of the properties of close binaries in different (extragalactic) stellar populations. The most thoroughly studied extragalactic system is M31, where more than 800 novae have been discovered over the past century (e.g., see Pietsch et al. 2007e; Shafter 2008, and references therein).

Despite this large number of novae discovered, very few follow-up studies of their photometric, or particularly their spectroscopic, properties have been attempted. Most recent M31 surveys have been undertaken through narrow-band filters in order to take advantage of the fact that novae fade more slowly in $\mathrm{H} \alpha$ than they do in the continuum (e.g., Ciardullo et al. 1987; Shafter \& Irby 2001). Such observations are ideal for determining the rate and spatial distribution of novae within a galaxy, but not for characterizing the nova light curves. As shown by Ciardullo et al. (1990b) the $\mathrm{H} \alpha$ light curves are not simply correlated with the peak luminosity as are the broad-band light curves. Moreover, most of the broadband light-curve data for M31 novae come from the early photographic studies of Arp (1956) and Rosino (1964, 1973), as summarized by Capaccioli et al. (1989), and from observations in Crimea and Latvia during the period between 1977 and 1990 (Sharov \& Alksnis 1992).

Similarly, spectroscopic data for M31 novae have, until recently, also been limited. The dearth of available spectra is not surprising given that novae in M31 are by definition transient, and relatively faint, reaching peak brightnesses in the range $m_{V} \approx 18$ to $m_{V} \approx 15 \mathrm{mag}$ before fading back to quiescence. Furthermore, since their eruptions are not predictable in advance, spectroscopic observations require not only timely access to large telescopes, but coordination with a photometric survey dedicated to discovering suitable targets. Humason (1932) was the first to report spectroscopic observations of classical novae in M31, and it was not until more than half a century later that Ciardullo et al. (1983) published the spectra 
of four $\mathrm{H} \alpha$ emission-line sources, which they identified as classical novae in eruption. The number of nova spectra has increased dramatically in recent years thanks to greater access to queue scheduling on large telescopes, such as the Hobby-Eberly Telescope (HET).

In order to better understand the spectroscopic properties of novae in M31, and to study any variation with spatial position in the galaxy, we began a multi-year M31 nova survey in the Fall of 2006 using the HET. The program was motivated in large part by the work of Williams (1992), who realized that the spectra of Galactic novae (taken within a few weeks of eruption) can be divided into one of two principal spectroscopic types: Fe II and He/N. These types are believed to be related to fundamental properties of the progenitor binary such as the white dwarf mass. As part of our HET program, we have measured the spectra of 26 M31 novae (representing $\sim 1 / 3$ of the total for which spectra are available), making it the most significant spectroscopic follow-up study of Local Group novae since the pioneering work of Tomaney \& Shafter (1992). To supplement these data, we have included 21 M31 nova spectra obtained over the years with the Lick Observatory 3-m Shane reflector. Finally, in order to characterize the light curves, we have employed a variety of telescopes to acquire broad-band photometric measurements of many of the novae in our spectroscopic sample. Here, we report the results of our survey.

\section{Observations}

\subsection{Spectroscopy}

During the early part of our survey, optical spectra were obtained primarily with the Lick 3-m Shane reflector using the Kast double spectrograph (Miller \& Stone 1993), although the observation of M31N 1990-10b was taken with the older UV Schmidt spectrograph (Miller \& Stone 1987). Most spectra were acquired in one of two basic instrument configurations. One used the D55 dichroic beamsplitter to split the spectrum over 5200-5500 $\AA$, with blue light being passed through the 600/4310 grism and red light being reflected off a 300/7500 (or 600/5000) grating. The resulting combined spectra cover the range $\sim 3300-10000 \AA$ ( $\sim 3300-7900 \AA)$, with the $2.0^{\prime \prime}$-wide slit giving a spectral resolution of $\sim 6 \AA$ in the blue and $\sim 11 \AA(6 \AA)$ in the red. The other setup removed the beamsplitter and all light was sent to a $600 / 5000$ grating on the red side, providing $\sim 6 \AA$ resolution over the range $\sim 4300-7000 \AA$.

The data were reduced using standard techniques (e.g., Foley et al. 2003). Routine CCD processing and spectrum extraction were completed with $\mathrm{IRAF}^{1}$, and the data were extracted

\footnotetext{
${ }^{1}$ IRAF is distributed by the National Optical Astronomy Observatory, which is operated by the Associ-
} 
with the optimal algorithm of Horne (1986). We obtained the wavelength scale from loworder polynomial fits to calibration-lamp spectra. Small wavelength shifts were then applied to the data after cross-correlating a template sky to the night-sky lines that were extracted with the nova. Using our own IDL routines, we fit spectrophotometric standard-star spectra to the data in order to flux calibrate our spectra and to remove telluric lines (Wade \& Horne 1988; Matheson et al. 2000). A summary of the Lick observations is given in Table 1.

Most of the spectra in this survey, and all of the ones obtained in the past few years, were acquired with the Marcario Low-Resolution Spectrograph (LRS; Hill et al. 1998) on the HET. Initially, we employed the $g 2$ grating with a $2.0^{\prime \prime}$ slit and the GG385 order-blocking filter, covering $4275-7250 \AA$ at a resolution of $R \approx 650$. Later, to obtain more coverage at longer wavelengths, we opted to use the lower resolution $g 1$ grating with a $1.0^{\prime \prime}$ slit and the GG385 filter. This choice increased our wavelength coverage to 4150-11000 $\AA$ while yielding a resolution of $R \approx 600$. In practice, the useful spectral range of the $g 1$ grating is limited to $\lambda \lesssim 9000 \AA$ where the effects of order overlap are minimal. All HET spectra were reduced using standard IRAF routines to flat-field the data and to optimally extract the spectra. These were placed on relative flux scales through comparison with observations of spectrophotometric standards routinely used at the HET. The data were not corrected for absorption by the Earth's atmosphere, and telluric absorption features are visible in some of the spectra. A summary of the HET observations is given in Table 2.

Between the Lick and HET observations, we obtained a total of 53 spectra of M31 nova candidates (21 with the Shane and 32 with the HET). These data, shown in Figures 112, include 46 M31 novae (5 with two spectra each), and two long-period Mira variables originally thought to be novae. Because the observations were made under a variety of atmospheric conditions with the stellar image typically overfilling the spectrograph slit, our data can not be considered spectrophotometric. Thus, all spectra have been displayed on a relative flux scale. In the caption for each figure we have indicated the time elapsed between discovery of the nova and the date of our spectroscopy. Although the date of discovery does not necessarily reflect the time of maximum light, M31 has been monitored much more frequently over the past decade, and we estimate that the discovery date is likely within a few days of peak brightness in the majority of recent cases.

ation for Research in Astronomy, Inc. under cooperative agreement with the National Science Foundation. 


\subsection{Photometry}

To complement our spectroscopic survey, we were able to amass sufficient photometric observations to produce light curves for many of the novae in our survey. Our primary motivation was to measure nova fade rates $\left(t_{2}\right)$ that could then be correlated with other properties, such as spectroscopic class. The photometric data consist both of targeted (mostly $B$ and $V$-band) observations, which were obtained primarily with the Liverpool Telescope (LT; Steele et al. 2004) and the Faulkes Telescope North (FTN; Burgdorf et al. 2007), and survey images (mainly $R$ band), taken over the years with a variety of telescopes. The LT and FTN data were reduced using a combination of IRAF and Starlink software, calibrated using standard stars from Landolt (1992), and checked against secondary standards from Magnier et al. (1992), Haiman et al. (1994), and Massey et al. (2006).

Our extensive $R$-band observations were taken largely from the photometric database compiled by one of us (K.H.) as part of an ongoing program to monitor nova light curves in M31. These data include both survey and targeted images taken with various telescopes with diameters of $0.26-6 \mathrm{~m}$. Most of the images come from the $0.65-\mathrm{m}$ telescope of the Ondřejov Observatory (operated partly by the Charles University, Prague) and the 0.35-m telescope in the private observatory of K.H. at Lelekovice. Standard reduction procedures for raw CCD images were applied (bias and dark-frame subtraction and flat-field correction) using the SIMS ${ }^{2}$ and Munipack ${ }^{3}$ programs. Reduced images of the same series were coadded to improve the signal-to-noise ratio; the total exposure time of these series varied from a few minutes up to about one hour. To facilitate nova detection, the gradient of the galaxy background was flattened by the spatial median filter using SIMS. Photometric and astrometric measurements of the novae were then performed using "Optimal Photometry" (based on fitting of point-spread function profiles) in GAIA ${ }^{4}$ and APHOT (a synthetic aperture photometry and astrometry software package developed by M. Velen and P. Pravec at the Ondřejov Observatory; see Pravec et al. [1994]), respectively. Five to ten comparison stars were used for individual brightness measurements. $B, V$, and $R$ magnitudes for comparison stars located in the M31 field were taken from Massey et al. (2006). Finally, in the case of images taken using the Sloan Digital Sky Survey (SDSS) $g^{\prime}$ and $r^{\prime}$ filters, we computed $g^{\prime}$ and $r^{\prime}$ magnitudes for comparison stars from $B V R I$ magnitudes taken from Massey et al. (2006) using empirical color transformations between the SDSS ugriz system and the Johnson-Cousins UBVRI system published by Jordi et al. (2006). A summary of all of our

\footnotetext{
${ }^{2}$ http://ccd.mii.cz/

${ }^{3}$ http: //munipack.astronomy.cz/

${ }^{4}$ http: //www.starlink.rl.ac.uk/gaia
} 
photometric observations is presented in Table 3.

Between our targeted and archival observations, we were able to produce light curves for a total of 47 of the 91 M31 novae with measured spectra (46 spectra from the present survey and 45 from the literature). The light curves are presented in Figures 13-20. Before turning to a discussion of this wealth of data, we briefly review our present understanding of nova populations, both in the Galaxy and in M31.

\section{Nova Populations}

\subsection{Background}

It has been conjectured, based both on Galactic and extragalactic observations, that there may exist more than one population of novae (e.g., Della Valle et al. 1992; Della Valle \& Livio 1998; Shafter 2008; Kasliwal et al. 2011, and references therein). Initially, Galactic observations suggested that novae associated with the disk were on average more luminous and faded more quickly than novae thought to be associated with the bulge (e.g., Duerbeck 1990; Della Valle et al. 1992). However, the interpretation of Galactic nova data is complicated by the need to correct for interstellar extinction, which can be significant and varies widely with the line of sight to a particular nova. Furthermore, extinction hampers the discovery of a significant fraction of objects: although Galactic novae occur at an estimated

rate of $\sim 30-35 \mathrm{yr}^{-1}$ (Shafter 1997, 2002), only about one in five of these are discovered and subsequently studied in any detail. Consequently, although much has been learned from the study of Galactic novae, it is clear that these data are not ideal for establishing the population characteristics of novae.

Nova eruptions are luminous enough to be detected as far away as the Virgo cluster. However, despite the considerable data amassed in recent years, evidence for distinct nova populations in extragalactic systems is conflicting. Ciardullo et al. (1990b) argued that a galaxy's nova rate was independent of the galaxy's Hubble type, and therefore independent of the galaxy's dominant stellar population. A few years later, based largely on the same data, but with different assumptions regarding the luminosity normalization, Della Valle et al. (1994) proposed that nova rates and light-curve properties (e.g., rate of decline from maximum light) did in fact vary between galaxies of differing Hubble types, with late-type systems such as M33 and the Magellanic Clouds having generally faster fading novae and higher luminosity-specific nova rates. However, subsequent studies (e.g., Shafter et al. 2000; Ferrarese et al. 2003; Shafter \& Williams 2004) have questioned these results, arguing that (given the considerable uncertainties that plague the determination of luminosity-specific 
nova rates) the available data are insufficient to establish any significant correlation. Further, the broader implications of the argument by Della Valle \& Duerbeck (1993) that novae in the Large Magellanic Cloud (LMC) generally were brighter and faded faster than those seen in the older stellar populations of the Galaxy or M31's bulge, has been called into question by Ferrarese et al. (2003) who showed that novae in M49, the first-ranked Virgo elliptical galaxy, generally faded at least as fast as novae in the LMC.

\subsection{The Spatial Distribution of Novae in M31}

The nearby Sb spiral, M31, is by far the most thoroughly studied extragalactic system, with observations of novae going back to Hubble (1929). Spanning $\sim 4$ deg on the sky, M31 is well resolved spatially, and offers a convenient target for the study of nova populations. In a classic nova survey from the mid 1950s, Arp (1956) reported the discovery of 30 M31 novae with the 60-in reflector at the Mount Wilson Observatory. Three principal conclusions of Arp's study [augmented by data from Hubble (1929)] were that (1) the frequency of novae dropped off more sharply than the galaxy's light, (2) the nova density decreased within $\sim 4^{\prime}$ of the nucleus, and (3) the frequency distribution of nova maximum magnitudes is bimodal, with peaks $m_{p g} \approx 16.0$ and $m_{p g} \approx 17.5$ mag (also see Capaccioli et al. 1989). A subsequent survey by Ciardullo et al. (1987) using $\mathrm{H} \alpha$ imaging confirmed that the nova spatial distribution is more centrally concentrated than the background light; in fact the distribution was consistent with a purely bulge population. The central "hole" in the nova distribution noted by Arp was not seen in the $\mathrm{H} \alpha$ data, and was assumed to be an artifact of the poor contrast against the bright nuclear background in Arp's photographic images.

Several more recent studies of the spatial distribution of M31 novae have confirmed the association with M31's bulge population (e.g., Capaccioli et al. 1989; Shafter \& Irby 2001; Darnley et al. 2004, 2006), but a major uncertainty in these studies is whether a significant fraction of disk novae are being missed due to internal extinction within the galaxy (see also Hatano et al. 1997). Taken at face value, the association of novae with M31's bulge population came as a surprise given that Galactic novae have long been recognized to have a significant disk population (e.g., Duerbeck 1984, 1990). To address this discrepancy, Ciardullo et al. (1987) proposed the idea that a significant fraction of the novae in M31's bulge could have been formed in the dense cores of the galaxy's globular clusters and subsequently been ejected into the bulge through three-body interactions within the clusters, through tidal disruption of some clusters, or a combination of both processes. 


\subsection{The Spectroscopic Classification of Galactic Novae}

A promising new approach for studying nova populations is to consider the character of a nova's spectrum within a few weeks after maximum light. When analyzing a large sample of Galactic nova spectra, Williams (1992) realized that the novae could be naturally segregated into two principal spectroscopic classes (Fe II and He/N) based on the emission lines in their spectra. Novae displaying prominent Fe II emission (the Fe II novae) usually show P Cygni absorption profiles, evolve more slowly, have lower expansion velocities, and show lower levels of ionization, compared to novae with strong lines of He and $\mathrm{N}$ (the $\mathrm{He} / \mathrm{N}$ novae). Complicating this division is the fact that a small fraction of novae initially exhibit Fe II emission lines along with broad (full width at half-maximum intensity [FWHM] $\gtrsim 2500 \mathrm{~km} \mathrm{~s}^{-1}$ ) Balmer emission before going on to develop spectra typical of the He/N novae. Such systems are referred to as either hybrid or Fe IIb novae. Both Fe II and $\mathrm{He} / \mathrm{N}$ novae (although more often the He/N systems) occasionally go on to develop strong Ne emission in their post-outburst spectra, which suggests that the higher-mass ONe white dwarfs may be found in both classes of novae.

Della Valle \& Livio (1998) analyzed the spatial distribution of a sample of 22 Galactic novae with data suitable for determining their spectroscopic class. By restricting their sample to novae with well-determined distances (i.e., from expansion parallax), they were able to use the observed positions to estimate the distance of each nova from the Galactic plane. They discovered that the fastest and brightest novae were primarily associated with the He/N spectroscopic class, and that the progenitors were preferentially located close to the Galactic plane (i.e., $z \lesssim 100$ pc). Fainter and dimmer novae, on the other hand, were more typically members of the Fe II class, and were found at much greater heights (up to $z \gtrsim 1000 \mathrm{pc}$ ). Thus, they concluded that the progenitors of the He/N novae were associated with a younger stellar population that are thought to contain a higher proportion of massive white dwarfs.

The precise mechanism that leads to the formation of two distinct post-eruption spectra is not well understood, but is thought to depend most sensitively on the mass of the white dwarf. Systems with relatively massive white dwarfs reach the critical density and temperature for a TNR after accreting a relatively small amount of hydrogen-rich material from the companion star (Starrfield et al. 2008; Townsley \& Bildsten 2005). Once the TNR takes

place, some fraction of this small accreted mass is ejected at high velocity in the form of a discrete shell. In systems with lower mass white dwarfs, more material is accreted prior to the TNR, and this larger amount of gas is ejected in a combination of a low-mass shell (early in the outburst), and an optically thick wind (shortly thereafter). It is this optically thick wind which is thought to be responsible for the formation of the $\mathrm{P}$ Cyg profiles seen in the lower excitation Fe II nova spectra. Thus, the fact that spectroscopic observations appear 
to offer a powerful discriminant between nova systems of varying white dwarf mass from differing stellar populations provided the principal motivation for our spectroscopic survey of novae in M31.

\section{The M31 Nova Survey}

\subsection{Spectroscopic Class}

Through the end of 2009, a total of 837 nova candidates have been discovered in M31 since the observations of Hubble (1929) began nearly a century ago.(Pietsch et al. 2007e) 5 Of these, spectra are now available for a total of 91 M31 novae, including the 46 from our present survey. As described above, novae spectra are, in principle, divisible into one of three primary classes: Fe II, He/N, and hybrid (or Fe IIb) novae. In practice, however, it is often difficult to make an unambiguous classification. Spectra are taken at different times after eruption, and the signal-to-noise ratio can vary widely from spectrum to spectrum. In addition, a nova can, on occasion, show characteristics of more than one class. For example, during the course of our spectroscopic survey, we have identified three novae (M31N 2007-10a, 2007-10b, and 2007-11b) that might have been traditionally classified as He/N or hybrid, but which do not share all of the characteristics of those classes. These spectra are dominated by prominent but narrow (FWHM $<2000 \mathrm{~km} \mathrm{~s}^{-1}$ ) lines of H, He I, and He II, with weaker N III and Fe II emission features occasionally seen. In the basic scheme of Williams (1992), such novae would be difficult to classify. Henceforth, we will refer to these objects as narrow-line $\mathrm{He} / \mathrm{N}$, or $\mathrm{He} / \mathrm{Nn}$, novae.

The spectra of all novae included in our survey (see Figs. 1-12) were examined and subsequently classified into one of six groups: Fe II, likely Fe II (Fe II:), He/N, likely He/N $(\mathrm{He} / \mathrm{N}:), \mathrm{He} / \mathrm{Nn}$, and hybrid (also known as broad-lined Fe II or Fe IIb novae). We found a total of 30 Fe II novae $(\sim 65 \%), 6$ likely Fe II novae $(\sim 13 \%), 3$ He/N novae $(\sim 6.5 \%), 3$ likely $\mathrm{He} / \mathrm{N}$ novae $(\sim 6.5 \%), 3 \mathrm{He} / \mathrm{Nn}$ novae $(\sim 6.5 \%)$, and 1 hybrid/Fe IIb nova $(\sim 2 \%)$. ${ }^{6}$ When all 91 novae with measured spectra are considered (see Table 4), the relative percentages remain similar with $\sim 74 \%$ (67 novae), $\sim 8 \%$ (7 novae), $11 \%$ (9 novae), $\sim 7 \%$ ( 6 novae), and $\sim 1 \%$ (one nova) representing the Fe II, Fe II:, He/N, He/N: (including the He/Nn systems),

\footnotetext{
${ }^{5}$ see also http://www.mpe.mpg.de/ m31novae/opt/m31/index.php

${ }^{6}$ Most, perhaps all, novae that are classified as He/N appear to display some weak Fe II emission near maximum light, and are therefore technically members of the hybrid class. Rather than referring to all of these novae as hybrid objects, we reserve the hybrid classification for those novae with prominent Fe II emission early on (e.g., M31N 2006-10b).
} 
and hybrid classes, respectively. Thus, when all the data are considered, approximately 4 out of $5(\sim 82 \%)$ of the total are likely Fe II novae, with the remaining systems falling in the $\mathrm{He} / \mathrm{N}$ and related $(\mathrm{He} / \mathrm{Nn}$ and hybrid/Fe IIb) classes.

There is some exiguous evidence that the relative percentage of $\mathrm{He} / \mathrm{N}$ and hybrid/Fe IIb novae may be somewhat higher in the Milky Way. In his original paper, Williams (1992) indicated that $\sim 40 \%$ of novae in his Galactic sample belonged to the He/N class. Similarly, Della Valle \& Livio (1998) found that as many as 10 out of $27(37 \%)$ in their sample of novae with well-determined distances (from expansion parallax) were $\mathrm{He} / \mathrm{N}$ or $\mathrm{Fe}$ IIb systems. More recently, however, Shafter (2007) has reviewed all available spectroscopic data for Galactic novae, finding that only 20 out of the 94 systems $(\sim 21 \%)$ with sufficient spectroscopic data available for classification appeared to be $\mathrm{He} / \mathrm{N}$ or hybrid systems. This is consistent with the fraction found in our M31 survey. The relatively large fraction of $\mathrm{He} / \mathrm{N}$ and Fe IIb systems included in the Della Valle \& Livio (1998) study, in particular, may be the result of their sample selection, which was restricted to novae with distances determined from expansion parallax. Such novae are more likely to be nearby and thus located in the Galactic disk.

\subsubsection{The Spatial Distribution of Spectroscopic Class}

The apparent concentration of $\mathrm{He} / \mathrm{N}$ and hybrid/Fe IIb novae toward the disk of the Galaxy (Della Valle \& Livio 1998) is an intriguing finding, and it would be of considerable interest if it could be confirmed in M31. While it is not possible to determine the height of a given nova above M31's galactic plane, we can explore differences in the spatial distributions between the different spectroscopic classes. Based on the Galactic results of Della Valle \& Livio (1998), one might expect that the He/N and related systems would be preferentially associated with the disk of M31, while the Fe II systems would perhaps display a more centrally concentrated, bulge-like distribution.

In Figure 21 we have plotted the projected positions of the 91 M31 novae with known spectroscopic class. Despite the expectation that the $\mathrm{He} / \mathrm{N}$ nova distribution might be more extended compared to the Fe II systems, there appears to be no obvious dependence of spectroscopic type with spatial position in the galaxy. This impression can be misleading, however, since the high inclination of M31 to the plane of the sky $\left(i \approx 77^{\circ}\right)$ makes it difficult to assign an unambiguous position within M31 to a given nova. This is particularly true for novae near the center of M31 where the foreground disk is superimposed on the galactic bulge. On the other hand, novae observed at a large galactocentric radius $\left(z 15^{\prime}\right)$ are likely to be associated with the disk of the galaxy. In order to approximate the true position of a 
nova within M31, we have assigned each nova an isophotal radius, defined as the length of the semimajor axis of an elliptical isophote [computed from the $R$-band surface photometry of Kent (1987)] that passes through the observed position of the nova.

In Figure 22 we show the cumulative distributions of the Fe II and He/N (and hybrid) novae plotted as a function of their isophotal radius. Although it appears that the He/Nhybrid distribution may be slightly more extended than the Fe II distribution, a KolmogorovSmirnov (KS) test reveals that the distributions would be expected to differ by more than that observed $81 \%$ of the time if they were drawn from the same parent population. When only a subset of the novae with well-established spectroscopic types are considered, this probability decreases slightly to $73 \%$.

The interpretation of these results is complicated by the fact that our spectroscopic data are drawn from a sample of M31 novae that may not be spatially complete. As mentioned earlier, although the CCD surveys conducted in $\mathrm{H} \alpha$ are essentially complete in the innermost regions of the galaxy, they did not typically cover the full disk of M31. In recent years the situation has improved with the availability of wide-field surveys, such as the ROTSE-IIIb program, which have provided good coverage over most of the galaxy. Given the nature of the M31 surveys, we suspect that our spectroscopic sample may be biased somewhat toward novae at smaller galactocentric radii (where, historically, the galaxy has been more frequently monitored), and thus may favor one spectroscopic class over the other. Nevertheless, such a bias should not affect the distributions of Figure 22 in a differential sense: both Fe II and $\mathrm{He} / \mathrm{N}$ novae are detectable throughout the galaxy. Another potential source of bias involves the fact that the $\mathrm{He} / \mathrm{N}$ novae are on average brighter and faster fading than the Fe II systems (see $§ 4.2 .1$ below). One could argue that the brighter $\mathrm{He} / \mathrm{N}$ novae might be easier to detect against the bright background of the bulge. However, this advantage would be offset to some degree by the fact that these novae generally fade more quickly, making them more likely to be missed in synoptic surveys. Consequently, we conclude, as did Di Mille et al. (2010) based on a smaller sample of novae, that there is no compelling evidence from the observed spatial positions of the novae in our sample that the Fe II and $\mathrm{He} / \mathrm{N}$ novae arise from different stellar populations in M31.

\subsubsection{Nova Expansion Velocity}

One of the defining properties of the $\mathrm{He} / \mathrm{N}$ spectroscopic class is that the emission-line widths are considerably broader than those seen in the Fe II novae. Specifically, Williams (1992) found that the emission lines of Galactic novae in the He/N class are characterized by a half-width at zero intensity $(\mathrm{HWZI})>2500 \mathrm{~km} \mathrm{~s}^{-1}$. Empirically, we have found that for 
most nova line profiles, the HWZI roughly equals the FWHM; since the latter is the more easily measured quantity, we have adopted it to characterize the spectra in our survey. The values of the FWHM and the equivalent widths of $\mathrm{H} \alpha$ and $\mathrm{H} \beta$ in our nova spectra are given in Table 5.

Although the emission-line width is expected to be correlated with the expansion velocity of the nova ejecta, the FWHM does not necessarily yield the expansion velocity directly. In an idealized nova, the broad emission features typically seen in an $\mathrm{He} / \mathrm{N}$ system are believed to be formed mainly in a discrete, optically thin shell ejected at relatively high velocity from near the white dwarf's surface. In this case, the line profiles are expected to be flat-topped and nearly rectangular in appearance, with the FWHM closely approximating the ejection velocity of the shell. In the Fe II systems, however, the lines are mainly produced in a wind, which originates at a distance above the surface of the white dwarf that varies as the outburst evolves. Thus, the escape velocity for this wind is smaller than that at the white dwarf's surface. As a result, the expansion velocity (and hence line width) may depend on the time elapsed since eruption.

When comparing the emission-line widths of the novae in our sample, it must be kept in mind that our spectroscopic data were obtained at varying times after eruption, and thus do not necessarily reflect the relative expansion velocities accurately. Nevertheless, as Figure 23 illustrates, a clear difference between the ejection velocities of the two principal classes of novae (Fe II and $\mathrm{He} / \mathrm{N}$ ) is apparent. Without exception, the novae belonging to the $\mathrm{He} / \mathrm{N}$ class are characterized by $\mathrm{H} \alpha \mathrm{FWHM}>2500 \mathrm{~km} \mathrm{~s}^{-1}$, while the Fe II systems all have H $\alpha$ FWHM less than this value. Interestingly, although $\mathrm{He} / \mathrm{Nn}$ novae display prominent lines of helium as do the standard He/N novae, they are narrow-line objects that resemble a typical Fe II nova at times (e.g., see M31N 2007-11b in Fig. 8). In addition, they often do not display prominent lines of nitrogen as do the typical He/N systems.

\subsection{Photometric Properties}

To further explore the properties of the novae in our survey, whenever possible we have augmented our spectroscopic data with available photometric observations. Few light curves are available for the novae in our spectroscopic sample that erupted prior to the start of our HET survey in 2006. Nevertheless, when the entire set of spectroscopic novae is considered, we have sufficient photometric data to estimate decline rates for half of the sample.

A convenient and widely used parameterization of the decline rate is $t_{2}$, which repre-

sents the time (in days) for a nova to decline 2 mag from maximum light. According to the 
criteria of Warner (2008), novae with $t_{2} \lesssim 25$ days are considered "fast" or "very fast," with the slowest novae characterized by $t_{2}$ values of several months or longer. Rates of decline, and corresponding values of $t_{2}$, have been measured (for all novae with sufficient photometric coverage) by performing weighted linear least-squares fits to the declining portion of the light curves that extend up to 3 mag below peak. In an attempt to account for systematic errors in the individual photometric measurements, the weights used in the fits were composed of the sum of the formal errors on the individual photometric measurements plus a constant systematic error estimate of $0.1 \mathrm{mag}$. The net effect of including the systematic error component was a reduction of the relative weighting of points with small formal errors and a corresponding increase in the formal errors of the best-fit parameters and in the uncertainties in $t_{2}$ derived from them.

Because our photometric observations do not always begin immediately after discovery, and the date of discovery does not always represent the date of eruption, we have made two modifications to our photometric data in order to better estimate the light-curve parameters. First, when available, we have augmented our light-curve data with the discovery dates and magnitudes given in the catalog of Pietsch et al. (2007e). ${ }^{7}$ Second, for some novae we have modified (brightened) the peak magnitude slightly through an extrapolation of the declining portion of the light curve up to 2.5 days pre-discovery in cases where upper flux limits (within 5 days of discovery) are available. The light-curve parameters resulting from our analysis are given in Table 6.

\subsubsection{MMRD Relations}

If we adopt a distance modulus for M31 of $\mu_{0}=24.38$ mag (Freedman et al. 2001) and a foreground reddening, $E(B-V)=0.062 \mathrm{mag}$ (Schlegel et al. 1998), we can compute the absolute magnitude at maximum light, and thereby produce calibrated maximum-magnitude versus rate-of-decline (MMRD) relations. The MMRD relations (the peak absolute magnitude vs. $\left.\log \mathrm{t}_{2}\right)$ for the $B, V$, and $R$ light curves are shown in Figures 24, 25, and 26, respectively. These plots illustrate not only that the peak nova luminosity is correlated with the rate of decline (i.e., the brightest novae generally fade the fastest), as was first studied extensively by McLaughlin (1945) for Galactic novae, but that the He/N systems are typically among the brightest and fastest novae. Weighted, linear least-squares fits to our $B, V$,

\footnotetext{
${ }^{7}$ see also http://www.mpe.mpg.de/ m31novae/opt/m31/index.php
} 
and $R$-band ${ }^{8}$ data yield

$$
\begin{aligned}
& M_{B}=-9.75 \pm 0.11+(1.69 \pm 0.085) \log t_{2}, \\
& M_{V}=-9.78 \pm 0.10+(1.70 \pm 0.080) \log t_{2},
\end{aligned}
$$

and

$$
M_{R}=-10.89 \pm 0.12+(2.08 \pm 0.077) \log t_{2}
$$

respectively. The peculiar He/Nn object M31N 2007-10b, which has particularly scanty lightcurve coverage, has a relatively large uncertainty in the peak magnitude. For comparison, in Figure 24 we show the theoretical $M_{B}$ vs. $\log t_{2}$ relation of Livio (1992), while in Figure 25 we include the Galactic $M_{V}$ relation from Downes \& Duerbeck (2000):

$$
M_{V}=-11.32 \pm 0.44+(2.55 \pm 0.32) \log t_{2}
$$

The slope of our M31 $V$-band MMRD relation is shallower than that for the Galactic data, and the M31 data are also systematically fainter than expected from the best-fitting Galactic relations. The latter discrepancy, in particular, as well as some of the scatter generally seen in the MMRD relations, is likely due to the fact that we have only corrected the M31 data for Galactic foreground extinction, not for extinction internal to M31. Based on these comparisons, it appears that the M31 nova sample perhaps suffers as much as 0.5 mag of extinction from within M31 itself, especially in the disk of the galaxy. This value is consistent with an estimate of $A\left(r^{\prime}\right)=0.5$ mag adopted by Darnley et al. (2006) based on the mean for Sb galaxies (Holwerda et al. 2005). In addition, our estimates of maximum light are based upon the magnitude at discovery, which will underestimate the peak luminosity in some cases. If we divide the novae in our sample into two groups, those with isophotal radii $r \leq 10^{\prime}$ and those with $r>10^{\prime}$, we find that the latter sample is slightly fainter at peak by an average of $\sim 0.4 \mathrm{mag}(16.6 \pm 1.1 \mathrm{vs} .17 .2 \pm 0.9 \mathrm{mag})$, although the difference is not statistically significant.

In agreement with the results of the Galactic study by Della Valle \& Livio (1998), it appears that the He/N novae are on average "faster" than their Fe II counterparts. Indeed,

\footnotetext{
${ }^{8}$ The $R$-band MMRD relation includes $r$-band data for novae where $R$-band observations are not available. When both $R$ and $r^{\prime}$ observations are available for a given nova, only the more extensive data set is used.
} 
although our sample is dominated by Fe II systems, three of the four fastest novae are He/N or related $(\mathrm{He} / \mathrm{Nn})$ systems. On the other hand, with the exception of the He/Nn nova M31N 2007-10b, we do not find strong evidence for a significant population of fast, but relatively faint, novae that apparently do not follow the classic MMRD relation. As discussed by Kasliwal et al. (2011), it is possible that these novae arise from progenitors containing high $\dot{M}$ (hot) and relatively massive white dwarfs, similar to what is expected for recurrent novae. Perhaps such novae are related to the class of $\mathrm{He} / \mathrm{Nn}$ novae described earlier.

Given that the He/N novae generally fade more quickly than the Fe II systems, and that $\mathrm{He} / \mathrm{N}$ novae have significantly higher expansion velocities based on their emission-line widths, the expansion velocity should be inversely correlated with the light-curve parameter, $t_{2}$. In Figure 27 we have plotted the measured $t_{2}$ value (based on the $V$ band when possible) versus the measured $\mathrm{H} \alpha$ FWHM for the 25 novae in our survey where it is possible to measure both parameters. As expected, there is a clear trend of faster novae exhibiting higher expansion velocities. The one exception is the He/Nn nova, M31N 2007-10a, which apparently evolved quite quickly despite its relatively slow expansion velocity. Based on these data (excluding M31N 2007-10a), a weighted linear least-squares fit yields the following:

$$
\log t_{2}(\mathrm{~d})=6.84 \pm 0.10-(1.68 \pm 0.02) \log [\mathrm{H} \alpha \mathrm{FWHM}(\mathrm{km} / \mathrm{s})]
$$

This relation can be compared with a similar one for Galactic novae found by McLaughlin (1960). A major factor in the discrepancy between the two may arise because in McLaughlin's relation the expansion velocities are derived from the absorption-line minima (P Cyg profiles) measured near maximum light. Typically, such velocities are only $20 \%$ to $50 \%$ of those inferred from the emission-line FWHM. The scatter in our data, particularly for the slower novae, is probably due in part to the time dependence of the derived velocities, as referred to in $\$ 4.1 .2$ above.

\subsubsection{Spatial Distribution of Nova Speed Class}

The question of whether the photometric properties of novae in M31 (e.g., peak brightness, fade rate) vary with spatial position in the galaxy (and possibly with stellar population) has yet to be thoroughly studied. Most recent surveys, which have concentrated primarily on the discovery of novae either for the purpose of measuring the spatial distribution, the overall rate, or both, lack the high cadence required to produce useful nova light curves. Light-curve data, when available, often only cover $\mathrm{H} \alpha$ or the $R$ band where the relationship between fade rate and peak luminosity is weak or absent (e.g., Ciardullo et al. 1990b). As 
mentioned earlier, available broad-band nova data come largely from the photographic surveys of Arp (1956), Rosino (1964, 1973), and Sharov \& Alksnis (1992). As noted above, the observation by Arp (1956) that the apparent magnitude distribution for M31 appeared to be bimodal, with peaks corresponding to $M_{B} \approx-8.5$ and $M_{B} \approx-7.0$ mag at the distance of M31, was later taken as evidence for the existence of two nova populations (e.g., Capaccioli et al. 1989).

The available light-curve data from previous surveys can be used to augment the extensive photometry obtained as part of our survey to study the variation of nova speed class with spatial position in M31. In particular, Capaccioli et al. (1989) has summarized the light-curve properties (peak magnitude and rate of decline) for novae in the Hubble (1929), Arp (1956), and Rosino (1964, 1973) surveys. From this compilation, we have selected the "high quality" data from their Table VI to supplement the light curves given in Table 6. Using this combined data set, we have assembled values of $t_{2}$ for a total of 74 novae. Of these, 35 (approximately half the total) are characterized by $t_{2} \leq 25$ days, and were categorized as either "fast" or "very fast" according to the definition in Warner (2008). For our purposes we simply refer to this group as the "fast" novae. We refer to the remaining 35 novae with $t_{2}>25$ days as "slow" novae. Given the uncertainties involved in accurately measuring $t_{2}$, we did not restrict our sample of fade rates to any particular color or bandpass; however, when data were available in multiple colors, we chose the $t_{2}$ values based on $B$-band observations to be as consistent as possible with data from earlier surveys.

In Figure 28 we have plotted separately the spatial distributions of the "fast" and "slow" novae. It appears that the slower novae (red circles) are perhaps more centrally concentrated than the fast sample (blue squares). This impression is confirmed when we consider the cumulative distributions shown in Figure 29. A KS test reveals that the two distributions would be expected to differ by more than they do $23 \%$ of the time if they were drawn from the same parent distribution. Thus, our data are consistent with the notion that "faster" novae, both in the Galaxy (Duerbeck 1990; Della Valle et al. 1992) and in M31, are more associated with the disk population than are the slower novae. We caution, however, that selection effects could potentially complicate the interpretation of this result. As was pointed out earlier in our discussion of spectroscopic class, our nova sample is not likely to be spatially complete. It is possible that we may be preferentially missing faster novae in the outer regions of the galaxy where the temporal sampling of the surveys has perhaps been less frequent. If so, our conclusion that the faster novae appear to be more spatially extended would actually be strengthened. Taken at face value, our results suggest that the photometric characteristics of novae are likely affected by changes in the underlying stellar population. 


\subsection{Discussion of Selected M31 Novae}

Below we highlight several individual novae of particular significance. These objects have either have been detected as a super-soft X-ray source (SSS), or have been observed extensively, both photometrically and spectroscopically, or have some peculiarity that warrants further discussion.

\subsubsection{M31N 1993-10g and 1993-11c}

As part of a program of follow-up spectroscopy of Local Group transients, one of us (A.V.F.) obtained spectra of two novae in the bulge of M31 on 1993 Nov. 8 and 17 (UT dates are used throughout this paper). The positions of the two objects are near that of two novae discovered in the survey by Shafter \& Irby (2001), M31N 1993-10g and 1993-11c, which are separated by $\sim 49^{\prime \prime}$. Unfortunately, the original observing logs are no longer available, so we are unable to make an unambiguous connection between the two spectra and the two novae. Based on approximate coordinate information in the FITS header for the spectrum taken on 1993 Nov. 17, we have made the tentative assignments indicated in Figures 1 and 2. The spectrum we have associated with M31N 1993-11h is clearly that of an Fe II nova, while that of 1993-11c is less certain but consistent with an Fe II classification.

Both novae have been identified as possible recurrent nova candidates by Shafter \& Irby (2001). The position of M31N 1993-10g is coincident with 1964-01a to within 9.8", while that of $1993-11 \mathrm{c}$ is within $3.6^{\prime \prime}$ and $6.5^{\prime \prime}$ of $1967-12 \mathrm{a}$ and $1923-02 \mathrm{a}$, respectively. Given that the coordinates for novae discovered on photographic plates are not known precisely in many cases, these both appear to be plausible recurrent nova candidates. However, both novae are located only $\sim 2^{\prime}$ from the nucleus of M31 where the nova density is high, increasing the probability of a chance positional coincidence. For a given observed separation $s$, we can compute the probability of a chance coincidence, $P_{C}$, by considering the nova density in an annulus of area $A$, centered at the position of each nova. Specifically,

$$
P_{C}=1-\exp \left[\sum_{\mathrm{i}=1}^{\mathrm{n}-1} \ln (1-\mathrm{ix})\right]
$$

where $n$ is the number of novae in the annulus, and $x=\pi s^{2} / A$. In both cases of interest here, we find that $P_{C} \gtrsim 0.95$, making it highly likely that the two outbursts were a chance coincidence from separate objects, and therefore not from a recurrent nova. 


\subsubsection{M31N 1995-11e}

M31N 1995-11e was identified during the nova survey of Shafter \& Irby (2001), who first recorded the object on 1995 Nov. 28 at $m_{H \alpha}=18.1$ mag. The object evolved quite slowly, reaching $m_{H \alpha}=17.9$ mag after approximately a month before slowly fading. Then, on 2008 July 6.8, K. Nishiyama and F. Kabashima (Miyaki-Argenteus Observatory, Japan) found that the object had appeared again at $m \approx 18.6$ mag (unfiltered) before reaching $m \approx 18$ mag on Sep. 09. Our spectrum presented here (see Fig. 12) and originally reported by Shafter et al. (2008) clearly shows that the object is a long-period red variable star (i.e., a Mira variable), and not a nova.

\subsubsection{M31N 2001-10a}

M31N 2001-10a was discovered as part of the POINT-AGAPE (Darnley et al. 2004) and the Naini Tal microlensing surveys (Joshi et al. 2004). Our spectroscopic data and $r^{\prime}$-band photometry show that M31N 2001-10a was a relatively slowly evolving Fe II nova characterized by $t_{2}=73$ days. X-ray observations reported by Henze et al. $(2010,2011)$ show the nova to be a long-lived SSS that was still detectable more than $7 \mathrm{yr}$ post outburst. The long duration of the SSS phase is indicative of prolonged burning on the surface of the white dwarf. This is expected for the relatively large accreted mass associated with slowly evolving outbursts on a low-mass white dwarf.

\subsubsection{M31N 2005-01a}

M31N 2005-01a, discovered by Hornoch (2005) on 2005 Jan. 07.89, was a particularly luminous nova that was well covered photometrically near maximum light, reaching $R=$ 15.04 mag (see Fig. 14). Our spectrum (see Fig. 4), taken 8 days post discovery when the nova was near $R=15.3 \mathrm{mag}$, shows that the object was clearly an Fe II system. It appears to be one of a small number of luminous Fe II novae $(M \lesssim-9.0 \mathrm{mag})$ similar to M31N 2007-11d and 2009-10b (see Figs. 24-26).

\subsubsection{M31N 2005-07a}

M31N 2005-07a, discovered by K.H. on Jul 200527.909 at $R=18.4$ mag, reached $R=17.4 \mathrm{mag}$ on Jul 29.919. Our spectrum (see Fig. 4), taken $\sim 2$ days post discovery 
when the nova was near maximum, is characterized by narrow $\mathrm{H} \alpha$ emission and a number of extremely weak features that may include He and $\mathrm{N}$ emission. We tentatively classify the object as an Fe II: system, but it is possibly related to the He/Nn novae.

\subsubsection{M31N 2005-09b}

M31N 2005-09b was discovered in the outskirts of M31 by Quimby et al. (2005) on Sep. 1.23 using the 0.45-m ROTSE-IIIb telescope at the McDonald Observatory. The nova reached $m=16.5 \mathrm{mag}$ (unfiltered) a day later on Sep. 2.23. Optical spectra by Leonard (2005) and Pietsch et al. (2006) reveal moderately broad Balmer (H $\alpha$ : FWHM $\approx 2000$ $\mathrm{km} \mathrm{s}^{-1}$, HWZI $\approx 2200 \mathrm{~km} \mathrm{~s}^{-1}$ ), Fe II, Na D, and He I emission features. The nova is consistent with membership in the Fe II spectroscopic class; however, the emission-line width is at the high end of what is normally seen in Fe II novae, and the nova could be plausibly included in the Fe IIb or hybrid class.

\subsubsection{M31N 2006-09c}

M31N 2006-09c was discovered independently by Quimby (2006), K. Itagaki, P. Kušnirák, and K.H. on 2006 Sep. $18 .^{9}$ It was detected $\sim 150$ days post discovery by both the IRAC and IRS instruments on the Spitzer Space Telescope as part of an infrared survey of selected M31 novae recently conducted by Shafter et al. (2011). No evidence of an infrared excess characteristic of dust formation was apparent at the time of these observations. The nova was also detected as a weak SSS by Henze et al. (2011) and originally classified as a Fe II nova by Shafter et al. (2006). Our $R$-band photometry shows that $t_{2}=26$ days, indicating a moderate rate of decline typical of the Fe II class.

\subsubsection{M31N 2006-10a}

M31N 2006-10a was a relatively faint nova discovered at $R=18.7$ mag on 2006 Oct. 25.8 by K.H. ${ }^{10}$ Our observations (see Figs. 5 and 14) reveal the object to be a slowly evolving Fe II nova. It was also observed by Shafter et al. (2011) 110 days post discovery as part of

\footnotetext{
${ }^{9}$ see http://www. cbat.eps.harvard.edu/CBAT_M31.html\#2006-09c, and http://www.mpe.mpg.de/ m31novae/opt/m31/M31_table.html

${ }^{10}$ see http://www. cbat.eps.harvard.edu/CBAT_M31.html\#2006-09c
} 
their Spitzer survey. M31N 2006-10a showed the clearest evidence of the 10 novae observed by Spitzer for a near-infrared excess (in this case peaking at $\lambda \approx 4 \mu \mathrm{m}$ ), suggestive of dust formation. They went on to estimate the total mass of dust formed to be $\sim 2 \times 10^{-6} \mathrm{M}_{\odot}$ under the assumption that the dust was carbon based. Henze et al. (2011) found no evidence of X-ray emission from this nova during the time of their observations.

\subsubsection{M31N 2006-10b}

M31N 2006-10b was discovered independently by K. Itagaki on 2006 Oct. 31.583 and by R. Quimby and F. Castro at $m \approx 16.4$ mag on unfiltered CCD images taken 2006 Oct. 31.09. ${ }^{11}$ The time of maximum light is well constrained by Itagaki's image from Oct. 30.530 (limiting mag 20.0), which shows no evidence of the nova. Although the light curve is not complete near maximum light, the available evidence suggests that the nova faded quite rapidly, with estimates of $t_{2}(B)=21$ days and $t_{2}(V)=11$ days.

We obtained two spectra of the nova, the first $\sim 1$ day post discovery on 2006 Nov. 01, and the other $\sim 3$ weeks later on 2006 Nov. 23. M31N 2006-10b can be technically considered a "hybrid" nova given that Fe II emission was seen the day after discovery, but by the time of the second observation the spectrum had clearly evolved into that of a classic He/N nova. The object was observed 102 and 110 days post discovery by Shafter et al. (2011) with the Spitzer IRS and IRAC, respectively, but not detected with either instrument.

\subsubsection{M31N 2006-11a}

M31N 2006-11a is a typical Fe II nova (see Fig. 6) that was discovered by K. Itagaki at $m=17.4 \mathrm{mag}$ (unfiltered) on 2006 Nov. 25.494. ${ }^{12}$ The object was observed by Shafter et al. (2011) as part of their Spitzer survey. It was marginally detected by the IRAC, but not with the IRS, 86 and 77 days after discovery, respectively.

\footnotetext{
${ }^{11}$ see http://www. cbat.eps.harvard.edu/CBATM31.html\#2006-10b

12 see http://www. cbat.eps.harvard.edu/CBAT_M31.html\#2006-11a
} 


\subsubsection{M31N 2007-02b}

This nova was discovered by one of us (K.H.) on 2007 Feb. $03 .{ }^{13}$ It was classified as a likely hybrid nova by Pietsch et al. (2007a) based on possible He and N emission in the spectrum. Our spectrum, taken on 2007 Feb. 10.06, suggests the object is an Fe II system (see Fig. 7), although there appears to be a broader component in the $\mathrm{H} \alpha$ and (possibly) the $\mathrm{H} \beta$ emission lines that is often seen in the hybrid systems. The light curve measured in the $R$ band yields $t_{2}(R)=35$ days, which is typical of an Fe II nova but would be somewhat slow for a hybrid system. The object was also detected as a SSS by Henze et al. (2011), and was still detectable $2 \mathrm{yr}$ after eruption. Such a long active SSS phase is characteristic of a relatively large accreted mass. We conclude that the object was likely an Fe II nova.

\subsubsection{2. $M 31 N 200 \%-06 b$}

M31N 2007-06b was discovered by Quimby et al. (2007) as part of the ROTSE IIIb program at McDonald Observatory on 2007 Jun 19.4 at $m=16.8$ mag (unfiltered) and found to be spatially coincident with the M31 globular cluster Bol 111. The nova faded by at least 1 mag in $\sim 9$ days, suggesting $t_{2} \lesssim 18$ days. Spectroscopic observations by Shafter \& Quimby (2007) revealed the nova to be a member of the He/N spectroscopic class. The object was subsequently detected as a SSS by Pietsch et al. (2007b).

\subsubsection{M31N 2007-07f}

M31N 2007-07f was a slowly evolving nova discovered in the outskirts of M31 as part of the ROTSE-IIIb program by Yuan et al. (2007), and is apparently a member of the Fe II spectroscopic class (Quimby 2007, private communication). The object exhibited a slow rise to maximum light, reaching $m=17.7 \pm 0.3 \mathrm{mag}$ on 2007 Jul. 24.02. Approximately 203 days later the object was observed in the Spitzer survey of Shafter et al. (2011) and detected by the IRAC. As discussed by Shafter et al. (2011), 2007-07f showed evidence (although more

marginal than for M31N 2006-10a) for a weak infrared excess, consistent with that expected from dust grains formed in the ejecta.

\footnotetext{
${ }^{13}$ see http://www. cbat.eps.harvard.edu/CBAT_M31.html\#2007-02b
} 


\subsubsection{M31N 2007-08d}

This object was discovered by Pietsch et al. (2007c) at $R=18.7$ mag on 2007 Aug. 24.081. Our spectrum (see Fig. 7) and light curve (Fig. 15) reveal the object to be a member of the Fe II class with $t_{2} \approx 63$ days. The nova was included in the Shafter et al. (2011) Spitzer survey and marginally detected with the IRAC, but not the IRS, about 158 and 183 days post discovery, respectively.

\subsubsection{M31N 2007-10a}

M31N 2007-10a was discovered K. Itagaki on Oct. 5.606 and independently by Pietsch et al. ${ }^{14}$ The spectrum was classified by Gal-Yam \& Quimby (2007) as an Fe II nova, but our spectrum (see Fig. 7) reveals narrow Balmer, He I $\lambda \lambda 4921,5016,5876$, and He II $\lambda 4686$ emission, with only a trace of Fe II, possibly blended with He I at $5169 \AA$. M31N 2007-10a is the prototype of our proposed He/Nn spectroscopic class. The nova faded rapidly with $t_{2}(B) \approx t_{2}(V) \approx 7$ days, and was not subsequently detected as an SSS by Henze et al. (2011), nor as an infrared source in the Spitzer survey of Shafter et al. (2011).

\subsubsection{M31N 2007-10b}

M31N 2007-10b is the best example from our survey of a faint but fast nova similar to the class of novae discovered by Kasliwal et al. (2011). The nova was discovered by Burwiz et al. (2007) at $R=17.8 \mathrm{mag}$, who were able to constrain the time of maximum to within a day of 2007 Oct. 13.26, and independently by K.H. and P. Kušnirák. ${ }^{15}$ The nova faded unusually quickly: our $B, V$, and $R$ light curves suggest $t_{2} \approx 3-4$ days. Rau et al. (2007) classified the object as an $\mathrm{He} / \mathrm{N}$ nova based on a spectrum obtained $\sim 3$ days post discovery, and noted that the emission lines were unusually narrow (FWHM $\mathrm{H} \alpha=1450 \pm 100 \mathrm{~km} \mathrm{~s}^{-1}$ ) for this class. Based on the available photometric and spectroscopic data, we suggest that the object is a member of our proposed He/Nn class. Consistent with its rapid evolution, the object was detected by Henze et al. (2011) as a SSS with an X-ray duration of less than 100 days.

\footnotetext{
${ }^{14}$ see http://www. cbat.eps.harvard.edu/CBAT_M31.html\#2007-10a

${ }^{15}$ see http://www. cbat.eps.harvard.edu/CBAT_M31.html\#2007-10b
} 


\subsubsection{M31N 2007-11b}

M31N 2007-11b, our third example of an He/Nn nova, was discovered by Pietsch et al. (2007d) and independently by E. Ovcharov and A. Valcheva. ${ }^{16}$ Although our spectrum (see Fig. 8) appears to be quite similar to that of an Fe II nova (albeit with weak Fe II emission), subsequent spectra by Rau (2007) and Barsukova et al. (2007b) showed that the object quickly developed prominent, narrow (FWHM H $\alpha=1430 \pm 100 \mathrm{~km} \mathrm{~s}^{-1}$ ) Balmer, He I, and He II emission lines. Unlike the other He/Nn systems (M31N 2007-10a and 2007-10b), the light-curve evolution of $2007-11$ b was not particularly fast, with $t_{2}(B)=25$ days and $t_{2}(V)=45$ days, respectively.

\subsubsection{M31N 2007-11d}

M31N 2007-11d was an unusually bright and slowly rising nova discovered by K. Nishiyama and F. Kabashima on Nov. 17.57, and subsequently studied extensively by Shafter et al. (2009). The early spectrum was that of a classic Fe II system: narrow Balmer and Fe II emission lines flanked to the blue by pronounced P-Cyg absorption features. Another spectrum obtained $\sim 2$ weeks later revealed moderately broad Balmer and Fe II emission lines (FWHM H $\alpha=2260 \mathrm{~km} \mathrm{~s}^{-1}$ ) with weak He I and O I emission (see Fig. 8). The nova faded moderately rapidly $\left(t_{2}[V]=9.5\right.$ days $)$ for an Fe II nova, and was apparently not detected as an SSS source despite what must have been a relatively large accreted mass. It was also observed, but not detected, with the Spitzer IRS (Shafter et al. 2011).

\subsubsection{9. $M 31 \mathrm{~N} 2007-11 \mathrm{~g}$}

M31N 2007-11g was discovered by Ovcharov et al. (2007) on 2007 Nov 28.716 at $R \approx 18.7$ mag. Our spectrum, presented in Fig. 12 and originally reported by Shafter et al. (2007), clearly shows that the object is not a nova, but rather a long-period Mira variable in M31.

\subsubsection{M31N 200\%-12b}

M31N 2007-12b was a relatively bright and rapidly evolving $\mathrm{He} / \mathrm{N}$ nova. It was discovered on 2007 Dec. 9.53 by K. Nishiyama and F. Kabashima1 at $m=16.1-16.2 \mathrm{mag}$

\footnotetext{
${ }^{16}$ see http://www. cbat.eps.harvard.edu/CBAT.M31.html\#2007-11b
} 
(unfiltered), and independently by K.H. on Dec. 10.73 at $R=17.0$ mag. ${ }^{17}$ Subsequent spectroscopic observations revealed the object to be a rapidly declining $\left(t_{2}=8.7[B], 7.2[\mathrm{~V}]\right.$ days) He/N system (see Fig. 9 and Table 6). Initially, the object was thought to be a recurrent nova given its close proximity to the position of M31N 1969-08a; however, subsequent astrometry established that the two novae were, in fact, distinct objects.

Archival Hubble Space Telescope observations of the pre-outburst location of M31N 200712b (Bode et al. 2009) revealed the presence of a coincident stellar source with magnitude and color very similar to those of the Galactic recurrent nova RS Ophiuchi, where the red giant secondary star dominates the light at quiescence. This discovery, coupled with the rapid photometric evolution and the object's detection by the Swift satellite (Burrows et al. 2005) as an SSS (Kong \& Di Stefano 2008), were interpreted by Bode et al. (2009) as strong evidence that M31N 2007-12b is, nevertheless, a recurrent nova system.

\subsubsection{M31N 2007-12d}

M31N 2007-12d was discovered independently by Henze et al. (2007) and by K. Nishiyama \& F. Kabashima ${ }^{18}$ on 2007-Dec. 17.57. Although no detailed light-curve data exist, Henze et al. (2011) estimated a rapid decline with $t_{2} \approx 4$ days. Our spectrum (see Fig. 9) reveals the nova to be a classic He/N system with broad emission lines of $\mathrm{H}$, He, and N (FWHM $\mathrm{H} \alpha \approx 5000 \mathrm{~km} \mathrm{~s}^{-1}$ ) indicating a high expansion velocity. The nova was detected briefly $(<20$ days) as an SSS by Henze et al. (2011).

\subsubsection{M31N 2009-10b}

M31N 2009-10b was discovered by K.H. and P. Kušnirák on 2009 Oct. 9.986 (Hornoch 2009), and independently by K. Itagaki on Oct. $11.414 .{ }^{19}$. It was an unusually bright nova that reached $R=14.7 \mathrm{mag}$ (Hornoch et al. 2009b) before fading relatively rapidly $\left(t_{2} \approx 10\right.$ days in $B$ and $\left.V\right)$. Spectroscopic observations by Di Mille et al. (2009b) and Barsukova et al. (2009b) show conclusively that the nova belongs to the Fe II spectroscopic class, making it quite similar to M31N 2007-11d.

\footnotetext{
${ }^{17}$ see http://www. cbat.eps.harvard.edu/CBAT M31.html\#2007-12b

${ }^{18}$ see http://www. cbat.eps.harvard.edu/CBAT_M31.html\#2007-12d

${ }^{19}$ see http://www. cbat.eps.harvard.edu/CBAT_M31.html\#2009-10b
} 


\section{Conclusions}

Whether there exist two distinct populations of classical novae is an important but unanswered question. In an attempt to gain further insight, we have conducted a major spectroscopic survey of novae in the nearby galaxy M31. These data have allowed us to determine spectroscopic classes for a total of 46 novae, more than doubling the number previously available. Specifically, after combining our data with published spectra, we have now been able to compile a list of spectroscopic classes for a total of 91 novae that erupted prior to 2010. In addition, we have undertaken photometric observations of many of the recent novae in this group in order to measure their light curves (i.e., their peak brightness and rate of decline). Whenever possible, we have augmented our photometric observations with light-curve data from the literature.

Our combined spectroscopic and photometric survey has allowed us to explore the spatial distribution of novae in M31 to a greater extent than has been possible previously. An analysis of these data has enabled us to arrive at the following conclusions.

- As part of this survey we have found that $\sim 80 \%$ of the M31 novae with available spectra belong to the Fe II class. The remaining $\sim 20 \%$ are composed of novae whose spectra are characterized by $\mathrm{H}, \mathrm{He}$, and $\mathrm{N}$ emission lines, with Fe II emission features either weak or absent. Usually these latter systems display relatively broad (FWHM $\gtrsim 2500 \mathrm{~km} \mathrm{~s}^{-1}$ ) lines typical of the He/N spectroscopic class; however, a small fraction of these systems (e.g., M31N 2007-10a, 2007-10b, and 2007-11b) are characterized by relatively narrow line widths $\left(\right.$ FWHM $\lesssim 2000 \mathrm{~km} \mathrm{~s}^{-1}$ ). We refer to the latter systems as narrow-line $\mathrm{He} / \mathrm{N}$, or $\mathrm{He} / \mathrm{Nn}$, novae. The relative percentages of Fe II and He/N (and related) novae are similar to those found for Galactic novae (Shafter 2007; Della Valle \& Livio 1998).

- We have presented photometric observations with sufficient coverage to determine lightcurve parameters (peak brightness and rate of decline) for most of the novae in our spectroscopic survey, and for approximately half of all novae with known spectroscopic classifications. These data have allowed us to confirm that novae in the $\mathrm{He} / \mathrm{N}$ and $\mathrm{He} / \mathrm{Nn}$ classes have generally faster light-curve evolution than the more common Fe II objects. When the light-curve parameters for the entire sample are considered, we find that the brighter novae generally fade the fastest; they are consistent with an MMRD relation. Similarly, we find that the ejection velocities inferred from line widths are higher for the faster novae, as is the case for their Galactic counterparts.

- Under the assumption that there exist two separate populations of novae (bulge and disk populations), it is believed that the disk population, with their generally more massive white dwarfs, should produce novae that are on average brighter and faster than their counterparts 
in the bulge population (Duerbeck 1990; Della Valle et al. 1992). To test this prediction further, we have explored the photometric behavior (specifically $t_{2}$ ) of novae as a function of spatial position in M31. After supplementing photometric data from our survey with decline rates from the "high quality" light-curve sample given by Capaccioli et al. (1989), we were able to generate a sample of 74 M31 novae with well-determined fade rates. This sample was subsequently divided into two groups: those with $t_{2} \leq 25$ days (the "fast" sample) and those with $t_{2}>25$ days (the "slow" sample). A comparison of the spatial distributions for the two samples shows that the fast novae are in fact more spatially extended from the core of M31 than the slow novae, as expected in the two-population scenario.

- We have also explored the possibility that the spectroscopic class of M31 novae varies with spatial position in the galaxy, as would be expected if the $\mathrm{He} / \mathrm{N}$ and related novae contain more massive white dwarfs. Surprisingly, as shown in Figures 21 and 22, the spatial distribution shows only a hint that Fe II objects may be more centrally concentrated (i.e., associated with the bulge of M31), and there is no compelling evidence for a dependence on spectroscopic class. Specifically, a KS test shows a $81 \%$ probability that the Fe II and He/N distributions would differ more than what is observed if they were drawn from the same overall distribution. This result suggests that the average white dwarf mass in nova systems may not be as strongly dependent on spatial position (and hence stellar population) in M31 as suggested by the photometric data.

Taken together, our spectroscopic and photometric data do not provide compelling evidence in support of the hypothesis that there exist two populations of novae in M31. Nevertheless, our light-curve data can be interpreted as mildly suggestive of a weak dependence of nova-speed class on spatial position (stellar population) within the galaxy. Furthermore, our spectra are not inconsistent with the possibility that spectroscopic type may be sensitive to stellar population. Whatever sensitivity there may be, however, appears to be weak, if it exists at all.

A major step forward in the understanding of nova populations generally, and the spectroscopic classification specifically, will likely require additional spectra and light-curve data for novae erupting in galaxies spanning a range of morphological types. For example, a sample of light curves and spectra from novae arising in the extreme Population II environment of an elliptical galaxy will be particularly instructive. The Large Synoptic Survey Telescope, when it becomes operational, will generate a large sample of Virgo cluster nova light curves that will undoubtedly shed new light on the question of nova populations. Spectra of novae in Virgo cluster galaxies can then be obtained with low-resolution spectrographs currently available on 10-m class telescopes. 
The work presented here was made possible through observations obtained from facilities based throughout the world. Spectra were obtained with the Lick Observatory Shane 3-m telescope operated by the University of California and with the Marcario Low-Resolution Spectrograph on the Hobby-Eberly Telescope, which is operated by McDonald Observatory on behalf of the University of Texas at Austin, the Pennsylvania State University, Stanford University, the Ludwig-Maximillians-Universität, Munich, and the George-AugustUniversitát, Göttingen. Photometric observations were made using the Liverpool Telescope, which is operated on the island of La Palma by Liverpool John Moores University (LJMU) in the Spanish Observatorio del Roque de los Muchachos of the Instituto de Astrofisica de Canarias with financial support from the UK Science and Technology Facilities Council. Faulkes Telescope North (FTN) is operated by the Las Cumbres Observatory Global Telescope network. Data from FTN were obtained as part of a joint programme between Las Cumbres Observatory and the LJMU Astrophysics Research Institute. Photometric observations were also obtained at the Centro Astronómico Hispano Alemán (CAHA) Observatory at Calar Alto, operated jointly by the Max-Planck Institut für Astronomie and the Instituto de Astrofísica de Andalucía (CSIC), with the 6-m telescope of the Special Astrophysical Observatory (SAO) of the Russian Academy of Sciences (RAS), operated under the financial support of the Science Department of Russia (registration number 01-43), with the Vatican Advanced Technology Telescope (the Alice P. Lennon Telescope and the Thomas J. Bannan Astrophysics Facility), with the 1.3-m McGraw-Hill and the 2.4-m Hiltner telescopes at the MDM Observatory, with the 2.5-m Isaac Newton Telescope operated on the island of La Palma by the Isaac Newton Group in the Spanish Observatorio del Roque de los Muchachos of the Instituto de Astrofsica de Canarias, with the 2.1-m telescope of the Kitt Peak National Observatory, National Optical Astronomy Observatory, which is operated by the Association of Universities for Research in Astronomy (AURA), Inc., under cooperative agreement with the National Science Foundation, and with the 0.84-m telescope of the Observatorio Astronómico Nacional, San Pedro Mártir. We wish to thank the staff of all these facilities for their assistance in obtaining the observations reported here.

Finally, we would like to thank the following individuals for contributing images of M31: V. L. Afanasiev, Z. Bardon, M. Burleigh, P. Cagaš, S. Casewell, S. N. Dodonov, T. Farnham, A. Galád, J. Gallagher, P. Garnavich, J. Gorosabel, T. Henych, M. Jelínek, A. Karska, C. Kennedy, R. Khan, P. Kubánek, P. Kušnirák, D. Mackey, K. Morhig, B. Mueller, O. Pejcha, J. Prieto, N. Samarasinha, L. Šarounová, P. Šedinová, O. N. Sholukhova, K. Thorne, B. Tucker, M. Tukinská, A. Valeev, M. Wolf, and P. Zasche. We are also grateful to the following for assistance with the Lick spectroscopic observations and reductions: A. Coil, R. J. Foley, M. Ganeshalingam, S. Jha, L. C. Ho, J. Hoffman, D. C. Leonard, W. Li, M. Papenkova, F. J. D. Serduke, J. C. Shields, and J. M. Silverman. Photometric reduction 
software was kindly provided by P. Cagaš (SIMS), F. Hroch (Munipack), and M. Velen and P. Pravec (Aphot). This research has made use of the SIMBAD database, operated at CDS, Strasbourg, France, and of NASA's Astrophysics Data System Bibliographic Services. A.V.F.'s group at UC Berkeley is grateful for the financial support of the National Science Foundation (most recently through grant AST-0908886) and the TABASGO Foundation. A.W.S. is grateful to the University of Victoria for hospitality during a recent sabbatical leave while this work was being completed, and to the NSF for financial support through grants AST-0607682 and AST-1009566. 


\section{REFERENCES}

Arp, H. 1961, ApJ, 133, 869

Barsukova, E., et al. 2007, ATel, 1186, 1

Barsukova, E., Afanasiev, V., Fabrika, S., Valeev, A., Hornoch, K., \& Pietsch, W. 2009b, ATel, 2251

Barsukova, E., Sholukhova, O., Valeev, A., Fabrika, S., Goranskij, V., Pietsch, W., \& Hornoch, K. 2008, ATel, 1773

Barsukova, E., Valeev, A., Fabrika, S., Burwitz, V., \& Pietsch, W. 2007b, ATel, 1314

Barsukova, E., Valeev, A., Sholukhova, O., Medvedev, A., Hornoch, K., Pietsch, W., \& Fabrika, S. 2009a, ATel, 2205

Barth, A. J. 2001, in Astronomical Data Analysis Software and Systems X, ed. F. R. Harnden, Jr., F. A. Primini, \& H. E. Payne (San Francisco: ASP, Conf. Ser. Vol. 238), 385

Bryan, J., Filippenko, A. V., \& Shields, J. C. 1990, IAUC, 5135

Bryan, J., Wren, W., Filippenko, A. V., Matheson, T., \& Ho, L. C. 1992, IAUC, 5658

Burrows, D. N. et al. 2005, SSRv, 120, 165

Bode, M. F., \& Evans, A. 1982, MNRAS, 200, 175

Bode, M. F., \& Evans, A. 1983, MNRAS, 203, 285

Bode, M. F., \& Evans, A. 1989, in Classical Novae, 1st ed., ed. M. F. Bode \& A. Evans (Chichester: J. Wiley), 163

Bode, M. F., Darnley, M. J., Shafter, A. W., Page, K. L., Smirnova, O., Anupama, G. C., \& Hilton, T. 2009, ApJ, 705, 1056

Burgdorf, M. J., Bramich, D. M., Dominik, M., Bode, M. F., Horne, K. D., Steele, I. A., Rattenbury, N., \& Tsapras, Y. 2007, P\&SS, 55, 582

Burwitz, V., Pietsch, W., Updike, A., Hartmann, D., Milne, P., \& Williams, G. 2007, ATel, 1238

Capaccioli, M., Della Valle, M., Rosino, L., \& D’Onofrio, M. 1989, AJ, 97, 1622

Chornock, R., Silverman, J. M., George, M. R., \& Filippenko, A. V. 2008, ATel, 1708 
Ciardullo, R., Ford, H. C., \& Jacoby, G. H. 1983, ApJ, 272, 92

Ciardullo, R., Ford, H. C., Neill, J. D., Jacoby, G. H., \& Shafter, A. W. 1987, ApJ, 318, 520

Ciardullo, R., Shafter, A. W., Ford, H. C., Neill, J. D., Shara, M. M., \& Tomaney, A. B. 1990b, ApJ, 356, 472

Ciardullo, R., Tamblyn, P. Jacoby, G. H., Ford, H. C., \& Williams, R. E. 1990a, AJ, 99, 1079.

Ciroi, S., Di Mille, F., Rafanelli, P., \& Temporin, S. 2007, ATel, 1292

Darnley, M. J., et al. 2004, MNRAS, 353, 571

Darnley, M. J., et al. 2006, MNRAS, 369, 257

Della Valle, M., Bianchini, A., Livio, M., \& Orio, M. 1992, A\&A, 266, 232

Della Valle, M., \& Duerbeck, H. W. 1993, A\&A, 271, 175

Della Valle, M., \& Livio, M. 1998, ApJ, 506, 818

Della Valle, M., Rosino, L., Bianchini, A., \& Livio, M. 1994, ApJ, 287, 403.

Di Mille, F., Ciroi, S., Rafanelli, P., Navasardyan, H., \& Bufano, F. 2007, ATel, 1325

Di Mille, F., Orio, M., Ciroi, S., Bianchini, A., Rafanelli, P., \& Nelson, T. 2008a, AN, 331, 197

Di Mille, F., Orio, M., Ciroi, S., Bianchini, A., Rafanelli, P., \& Nelson, T. 2010, AN, 331, 197

Di Mille, F., Ciroi, S., Navasardyan, H., Orio, M., Rafanelli, P., \& Bianchini, A. 2009a, ATel, 2171

Di Mille, F., Ciroi, S., Orio, M., Rafanelli, P., Bianchini, A., Nelson, T., \& Andreuzzi, G. 2008b, ATel, 1818

Di Mille, F., et al. 2009, ATel, 2248

Downes, R. A., \& Duerbeck, H. W. 2000, AJ, 120, 2007

Duerbeck, H. W. 1984, Ap\&SS, 99, 363

Duerbeck, H. W. 1990, in Physics of Classical Novae, ed. A. Cassatella \& R. Viotti (New York: Springer-Verlag), 96 
Fabrika, S., Sholukhova, O., Valeev, A., Hornoch, K., \& Pietsch, W. 2009a, ATel, 2239

Fabrika, S., Sholukhova, O., Valeev, A., Hornoch, K., \& Pietsch, W. 2009b, ATel, 2240

Ferrarese, L., Côté, P., \& Jordán, A. 2003, ApJ, 599, 1302

Foley, R. J., et al. 2003, PASP, 115, 1220

Freedman, W. L. 2001, ApJ, 553, 47

Gal-Yam, A., \& Quimby, R. 2007, ATel, 1236

Hachisu, I., \& Kato, M. 2006, ApJS, 167, 59

Haiman, Z., et al., 1994, A\&A, 286, 725

Hatano, K., Branch, D., Fisher, A., \& Starrfield, S. 1997, ApJ, 487, L45

Hatzidimitriou, D., Reig, P., Manousakis, A., Pietsch, W., Burwitz, V., \& Papamastorakis, I. 2007, A\&A, 464, 1075

Henze, M., Pietsch, W., Updike, A., Hartmann, D., Milne, P., \& Williams, G. 2007, ATel, 1336

Henze, M., et al. 2010, A\&A, 523, 89

Henze, M., et al. 2011, A\&A, in press.

Hill, G. J., Nicklas, H. E., MacQueen, P. J., Tejada, C., Cobos Duenas, F. J., \& Mitsch, W. 1998, Proc. SPIE, 3355, 375

Holwerda, B. W., Gonzalez, R. A., Allen, R. J., \& van der Kruit, P. C. 2005, AJ, 129, 1396

Horne, K. 1986, PASP, 98, 609

Hornoch, K. 2005, IAUC, 8461.

Hornoch, K. 2009, CBET, 1971

Hornoch, K., \& Pejcha, O. 2009d, CBET, 2061

Hornoch, K., Pejcha, O., \& Kusnirak, P. 2009c, CBET, 2058

Hornoch, K., Pejcha, O., \& Wolf, M. 2009a, CBET, 2062

Hornoch, K., Pejcha, O., Zasche, P., \& Kusnirak, P. 2009b, CBET, 2057 
Hubble, E. P. 1929, ApJ, 69, 103

Humason, M. L. 1932, PASP, 44, 381

Jordi, K., Grebel, E. K., \& Ammon, K. 2006, A\&A, 460, 339

Joshi, Y. C., Pandley, A. K., Narasimha, D., Giraud-Héeraud, Y., Sagar, R., \& Kaplan, J. 2004, A\&A, 415, 471

Kasliwal, M. M. 2009, CBET, 2015

Kasliwal, M. M., Cenko, S. B., Kulkarni, S. R., Ofek, E. O., Quimby, R. \& Rau, A. 2011, ApJ, in press.

Kasliwal, M. M., Rau, A., Salvato, M., Cenko, S. B., Ofek, E. O., Quimby, R., \& Kulkarni, S. R. 2008, ATel, 1886

Kent, S. M. 1987, AJ, 95, 306

Kong, A. K. H., \& Di Stefano, R. D. 2008, ATel, 1360

Landolt, 1992, AJ, 104, 340

Livio, M. 1992, ApJ, 393, 516

Miller, J. S., \& Stone, R. P. S. 1987, Lick Observatory Technical Reports, Vol. 48 (Santa Cruz, CA: Lick Obs.)

Miller, J. S., \& Stone, R. P. S. 1993, Lick Observatory Technical Reports, Vol. 66 (Santa Cruz, CA: Lick Obs.)

Magnier, E. A. et al.,1992, A\&AS, 96, 379

Massey, P., et al., 2006, AJ, 131, 2478

Matheson, T., Filippenko, A. V., Ho, L. C., Barth, A. J., \& Leonard, D. C. 2000, AJ, 120, 1499

Medvedev, A., et al. 2009, ATel, 2213

McLaughlin, D. B. 1945, PASP, 57, 69

McLaughlin, D. B. 1960, in Stellar Atmospheres, ed. J. L. Greenstein (Chicago: Univ. of Chicago Press) 
Ovcharov, E., Valcheva, A., Kostov, A., Nikolov, Y., Georgiev, Ts., \& Nedialkov, P. 2007, ATel, 1312

Pietsch, W., Burwitz, V., Stoss, R., \& Sanchez, S. 2005, ATel, 520

Pietsch, W., et al. 2007a, ATel, 1009

Pietsch, W., Burwitz, V., Greiner, J., Haberl. F, Henze, M., \& Sala, G. 2007b, ATel, 1294

Pietsch, W., Burwitz, V., Stefanescu, A., Hatzidimitriou, D., Ppsel, J., Binnewies, S., Ruder, H., Papamastorakis, G. 2007c, ATel, 1201

Pietsch, W., Burwitz, V., Updike, A., Milne, P., Williams, G., \& Hartmann, D. 2007d, ATel, 1257

Pietsch, W., et al., 2007e, A\&A, 465, 375

Quimby, R. 2006 ATel, 887

Quimby, R., Mondol, P., Hoeflich, P., Wheeler, J. C., \& Gerardy, C. 2005, ATel, 600

Quimby, R., et al. 2007, ATel, 1118

Rau, A. 2007, ATel, 1276

Rau, A., Burwitz, V., Cenko, S. B., Updike, A., Hartmann, D., Milne, P., \& Williams, G. 2007b, ATel, 1242

Rau, A., Burwitz, V., Hatzidimitriou, D., \& Cenko, S. B. 2007a, ATel, 1153

Rau, A., \& Cenko, S. B. 2007, ATel, 1331

Rau, A., Kasliwal, M. M., \& Burwitz, V. 2008, ATel, 1568

Reig, P., Primak, N., Akras, S., Hatzidimitriou, D., Pietsch, W., \& Papamastorakis, G. 2008, ATel, 1612

Rodríguez-Gil, P., Ferrando, R., Rodríguez, D., Bode, M. F., Huxor, A., Giles, P., \& Mackey, D. 2009, ATel, 2166

Rosino, L. 1964, AnAp, 27, 498

Rosino, L. 1973, A\&AS, 9, 347

Schlegel, D. J., Finkbeiner, D. P., \& Davis, M. 1998, ApJ, 500, 525 
Shafter, A. W. 1997, ApJ, 487, 226

Shafter, A. W. 2002, in Classical Nova Explosions, ed. M. Hernanz \& J. José (New York: AIP, Conf. Proc. 637)

Shafter, A. W. 2007, BAAS, 211.5115

Shafter, A. W. 2008, in Classical Novae, 2nd ed., ed. M. Bode \& A. Evans (Cambridge: Cambridge Univ. Press), 335

Shafter, A. W., Bode, M. F., Darnley, M. J., Misselt, K. A., Rubin, M., \& Hornoch, K. 2011, ApJ, 727, 50

Shafter, A. W., Ciardullo, R., Bode, M. F., Darnley, M. J., Misselt, K. A., Nishiyama, K., \& Kabashima, F. 2008 ATel, 1834

Shafter, A. W., Ciardullo, R., \& Pritchet, C. J. 2000, ApJ, 530, 193

Shafter, A. W., Coelho, E. A., Misselt, K. A., Bode, M. F., Darnley, M. J., \& Quimby, R. 2006, ATel, 923

Shafter, A. W., \& Irby, B. K. 2001, ApJ, 563, 749

Shafter, A. W., Rau, A., Quimby, R. M., Kasliwal, M. M., Bode, M. F., Darnley, M. J., \& Misselt, K. A. 2009, ApJ, 690, 1148

Shafter, A. W. \& Quimby, R. M 2007, ApJ, 671, 121

Shafter, A. W., \& Williams, S. J. 2004, ApJ, 612, 867

Shafter, A. W., et al. 2007, ATel, 1851.

Sharov, A. S. 1993, Ast.L., 19, 7

Sharov, A. S., \& Alksnis, A. 1992, Ap\&SS, 190, 119

Starrfield, S., Iliadis, C., \& Hix, R. 2008, in Classical Novae, 2nd ed., ed. M. Bode \& A. Evans (Cambridge: Cambridge Univ. Press), 77

Steele, I. A., et al., 2004, SPIE, 5489, 679

Tomaney, A. B., \& Shafter, A. W. 1992, ApjS, 81, 683

Townsley, D. M., \& Bildsten, L. 2005, ApJ, 628, 395 
Tutukov, A. V., \& Yungelson, L. R. 1995, in Cataclysmic Variables, ed. A. Bianchini, M. Della Valle, \& M. Orio (Dordrecht: Kluwer), 495

Valeev, A., Barsukova, E., Sholukhova, O., Medvedev, A., Hornoch, K., Kusnirak, P., Pietsch, W., \& Fabrika, S. 2009, ATel, 2208

Wade, R. A., \& Horne, K. 1988, ApJ, 324, 411

Warner, B. 1995, in Cataclysmic Variable Stars, (Cambridge: Cambridge Univ. Press)

Warner, B. 2008, in Classical Novae, 2nd ed., ed. M. Bode \& A. Evans (Cambridge: Cambridge Univ. Press), 16

Williams, R. E. 1992, AJ, 104, 725

Yuan, F., Rykoff, E., Aretakis, J., Akerlof, C., \& Quimby, R. 2007, ATel, 1158 


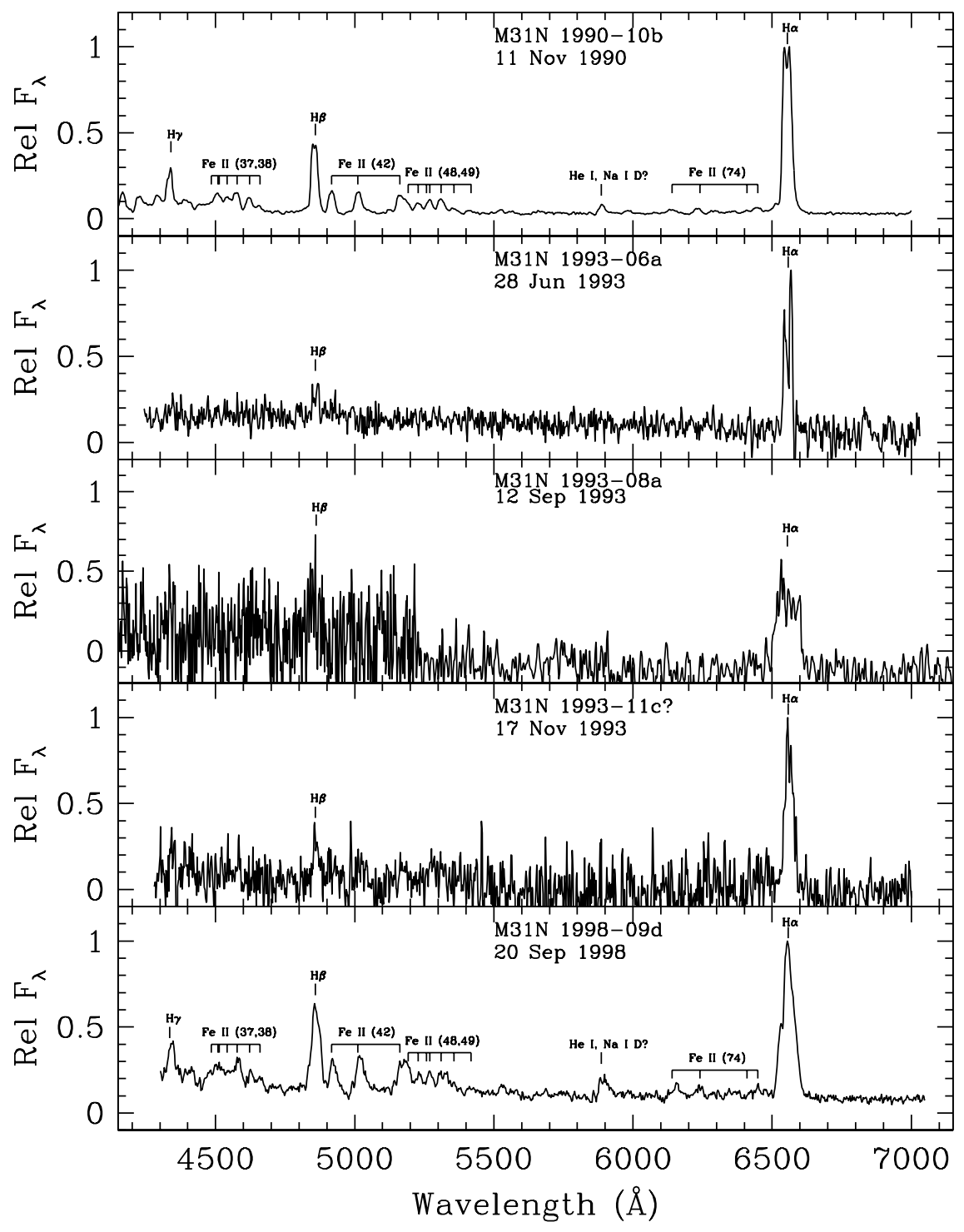

Fig. 1.- Spectra of the M31 novae M31N 1990-10b, 1993-06a, 1993-08a, 1993-11c (ID uncertain; see text), and 1998-09d, taken 19, 25, 37, 9, and 9 days post discovery, respectively. All are Fe II systems with the possible exception of M31N 1993-08a, which has a broad H $\alpha$ emission line characteristic of the $\mathrm{He} / \mathrm{N}$ novae. 


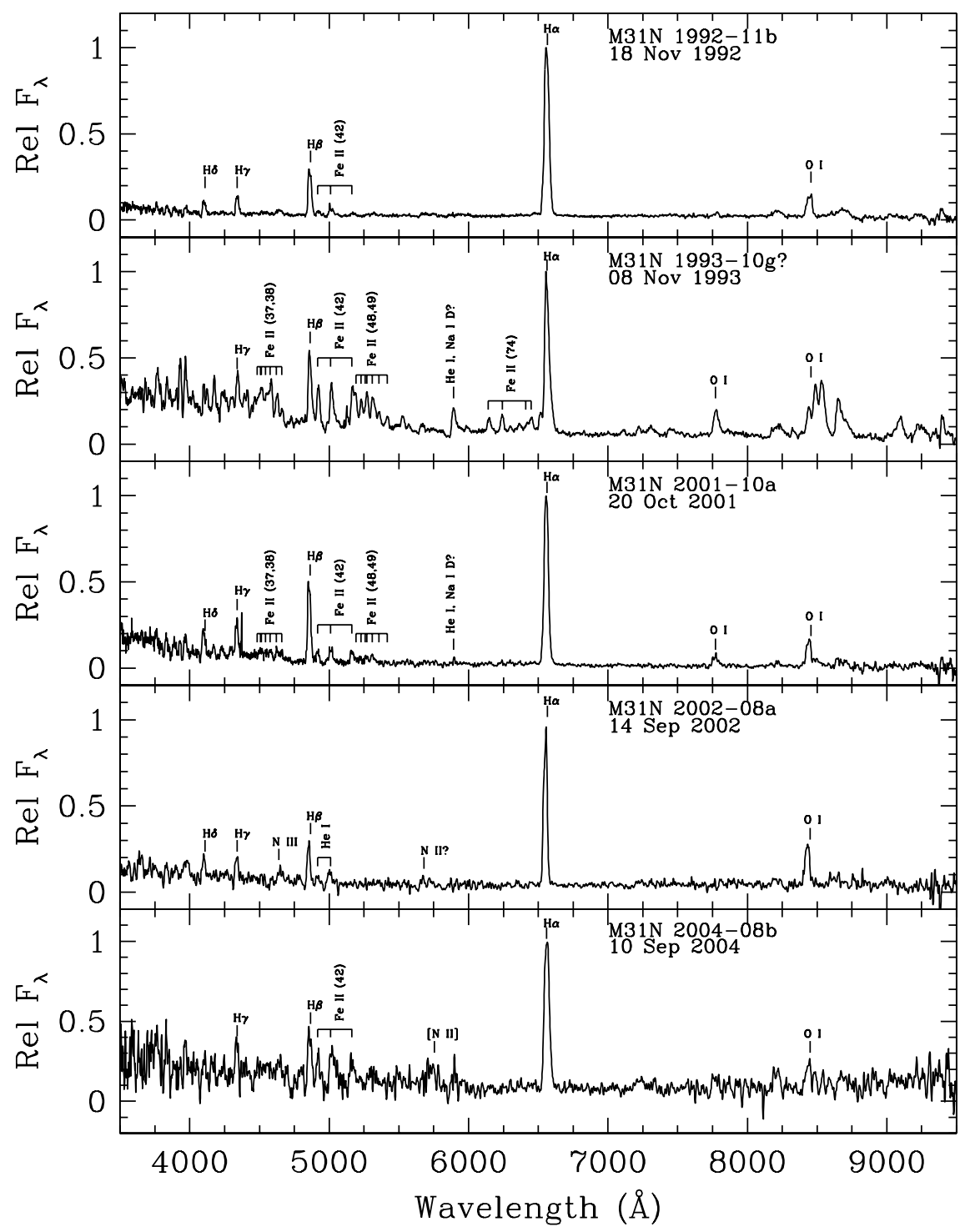

Fig. 2.- Spectra of the M31 novae M31N 1992-11b, 1993-10g (ID uncertain, see text), 200110a, 2002-08a, and 2004-08b obtained 10, 21, 17, 40, and 34 days post discovery, respectively. All are Fe II systems with the possible exception of 2002-08a, which was observed well past maximum light and has a spectrum similar to that of an $\mathrm{He} / \mathrm{Nn}$ nova. 


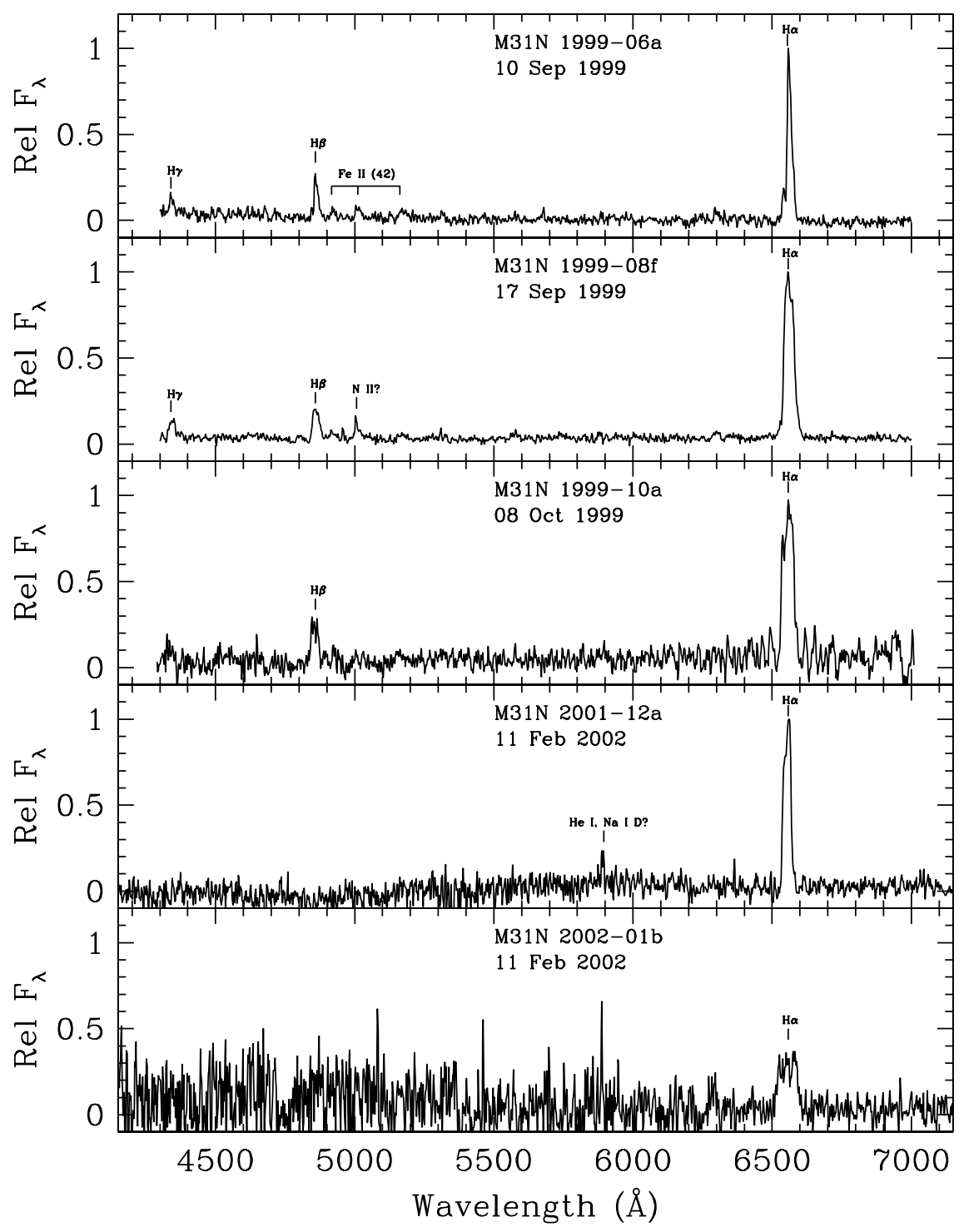

Fig. 3.- Spectra of the M31 novae M31N 1999-06a, 1999-08f, 1999-10a, 2001-12a, and 200201b, taken 75, 19, 6, 58, and 35 days post discovery, respectively. All are Fe II novae with the exception of 1999-08f, where the type is uncertain, and 2002-01b, where the broad $\mathrm{H} \alpha$ line suggests that the object may be a $\mathrm{He} / \mathrm{N}$ system. 


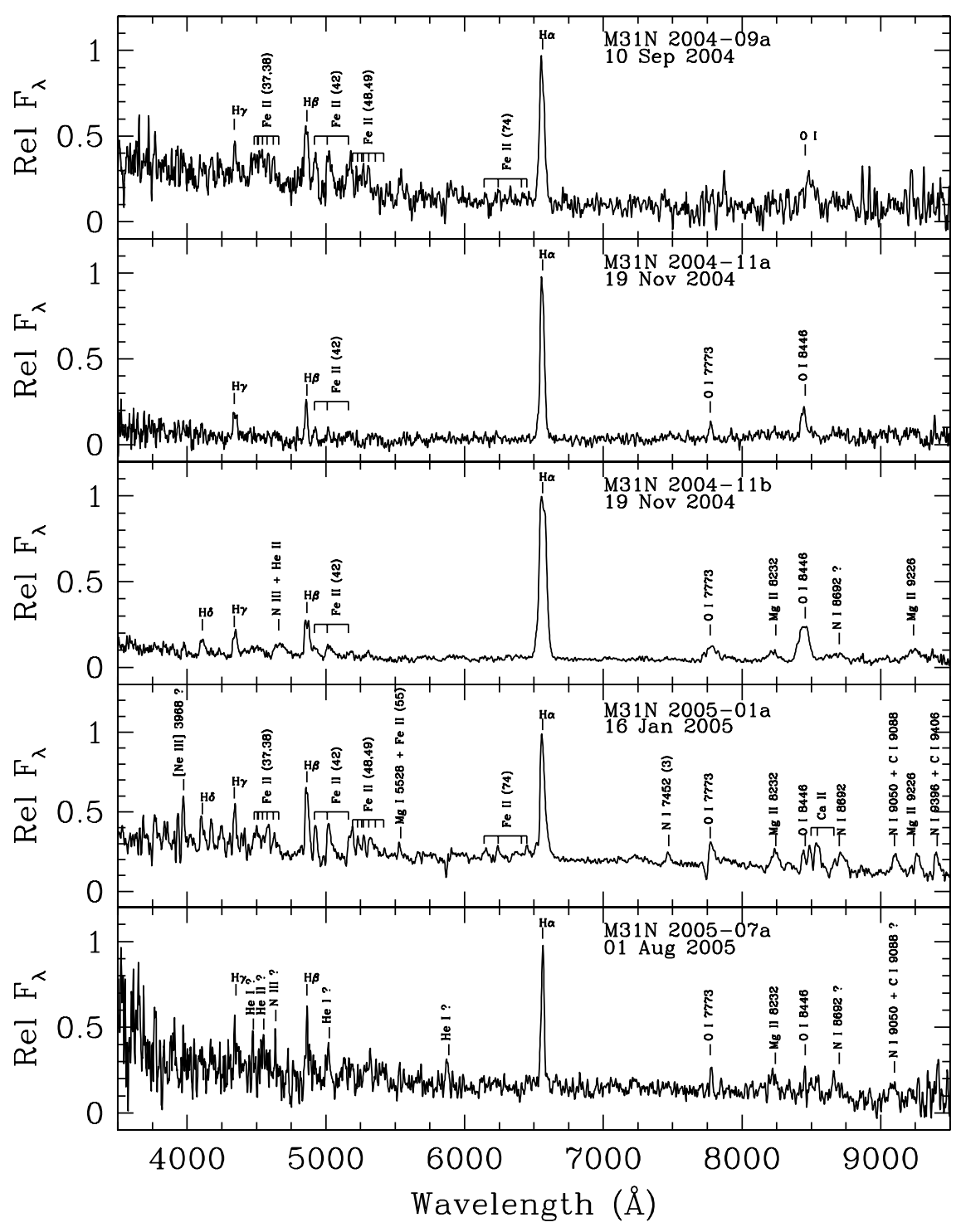

Fig. 4.- Spectra of the M31 novae M31N 2004-09a, 2004-11a, 2004-11b, 2005-01a, and 2005-07a taken 8, 14, 14, 8, and 2 days post discovery, respectively. All are Fe II novae with the possible exception of 2004-11b, which has a spectrum characterized by relatively broad Balmer and N III emission similar to that of an Fe IIb, or hybrid nova, and M31N 2005-07a, which might be a $\mathrm{He} / \mathrm{Nn}$ system. 


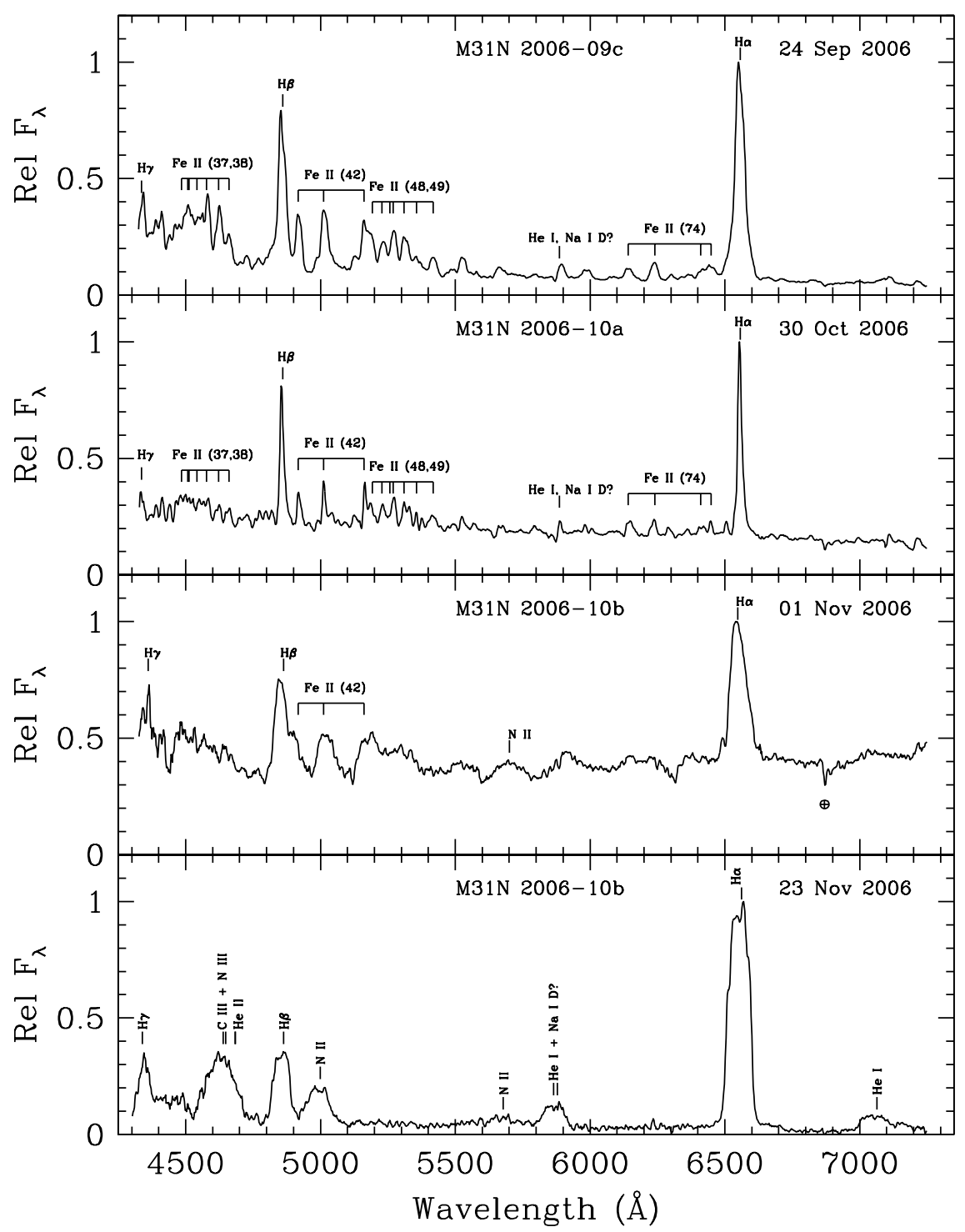

Fig. 5.- Spectra of the M31 novae M31N 2006-09c, 2006-10a, and two observations of 2006-10b taken 7, 8, 2, and 24 days post discovery, respectively. Both M31N 2006-09c and M31N 2006-10a are typical Fe II novae, while M31N 2006-10b (observed twice) is an example of a hybrid or Fe IIb nova. By the time of the second spectrum, M31N 2006-10b had evolved into a classic $\mathrm{He} / \mathrm{N}$ nova. 


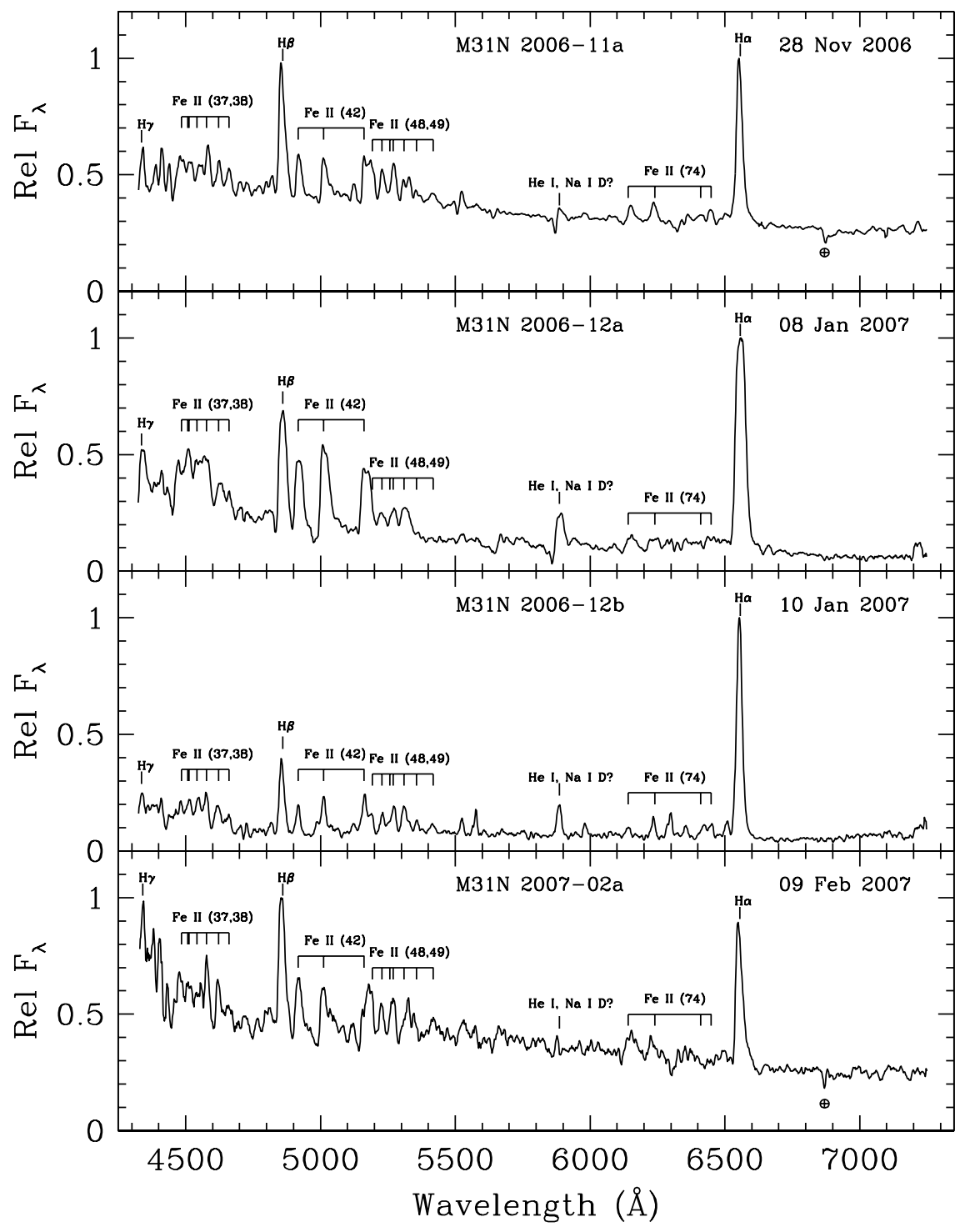

Fig. 6. - Spectra of the M31 novae M31N 2006-11a, 2006-12a, 2006-12b, and 2007-02a, taken 4, 24, 18, and 3 days post discovery, respectively. All four novae are typical members of the Fe II class. 


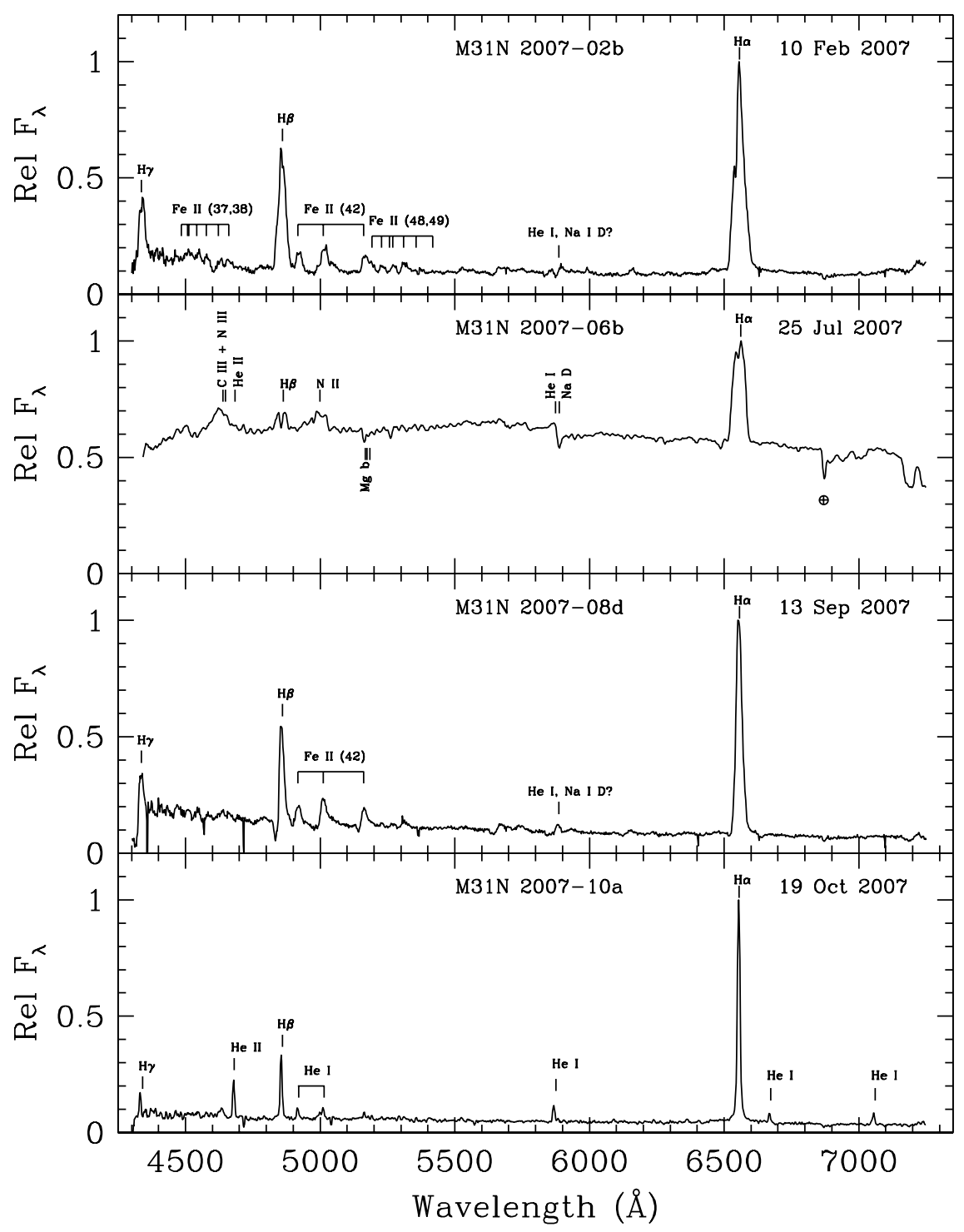

Fig. 7.- Spectra of the M31 novae M31N 2007-02b, 2007-06b, 2007-08d and 2007-10a, taken $7,37,21$, and 14 days post discovery, respectively. M31N 2007-02b is likely to be an Fe II system, although the broad component in the Balmer lines is often seen in hybrid novae. M31N 2007-06b is a He/N nova that originated in the M31 globular cluster Bol 111 (Shafter \& Quimby 2007). M31N 2007-08d is a Fe II system. M31N 2007-10a is an unusual nova displaying prominent Balmer and He I lines. The nova is not typical of either the Fe II class (no Fe II lines) or the He/N class (the lines are narrow and there is no sign of nitrogen lines). This is the prototype of our new class of the narrow-lined He novae ( $\mathrm{He} / \mathrm{Nn})$. 


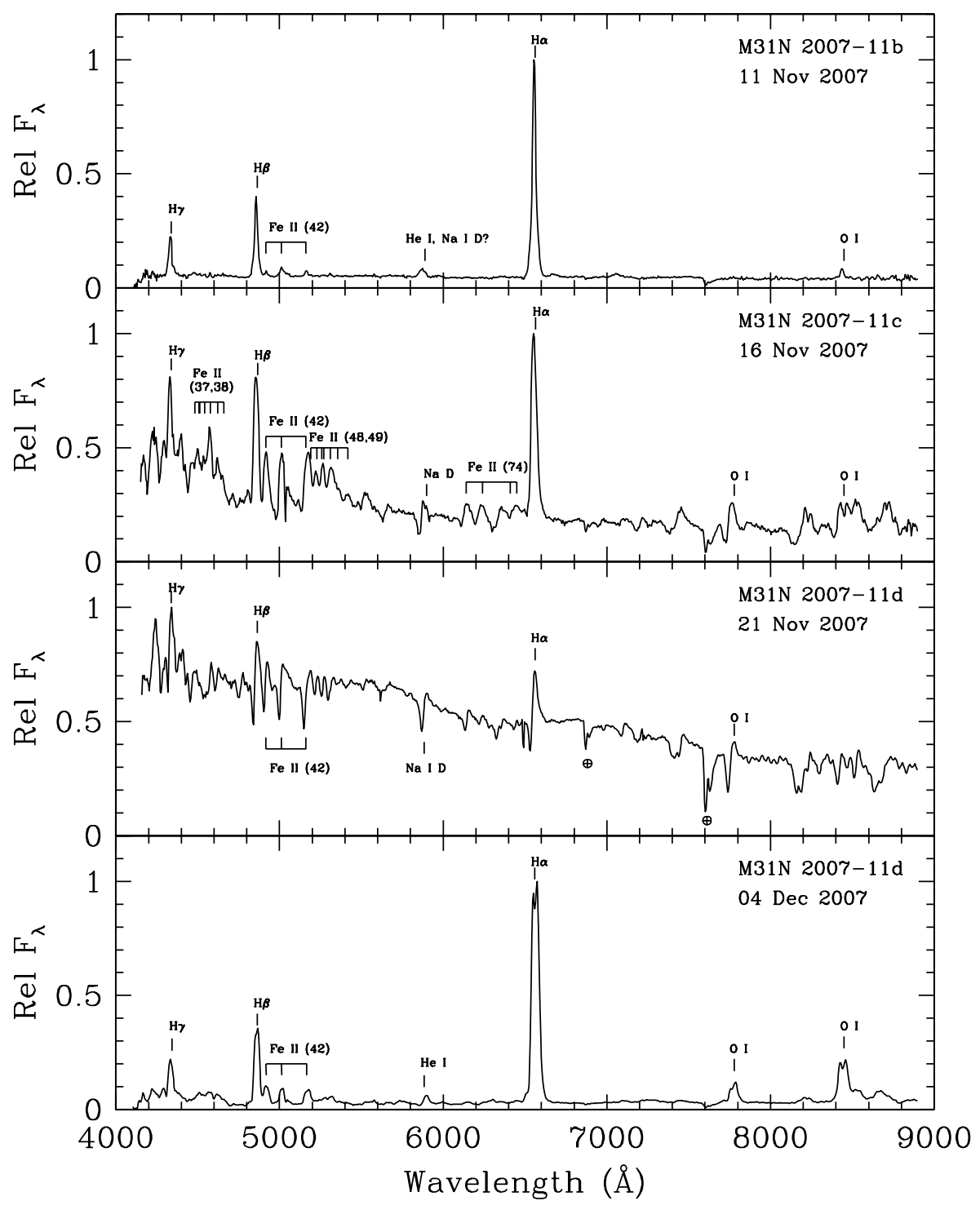

Fig. 8.- Spectra of the M31 novae M31N 2007-11b, 2007-11c, and 2007-11d (two spectra), taken 11, 5, 5, and 18 days post discovery, respectively. All are Fe II novae. M31N 2007-11d was observed twice, shortly after eruption when the P Cyg line profiles were clearly evident, and roughly two weeks later after the continuum had faded considerably. 


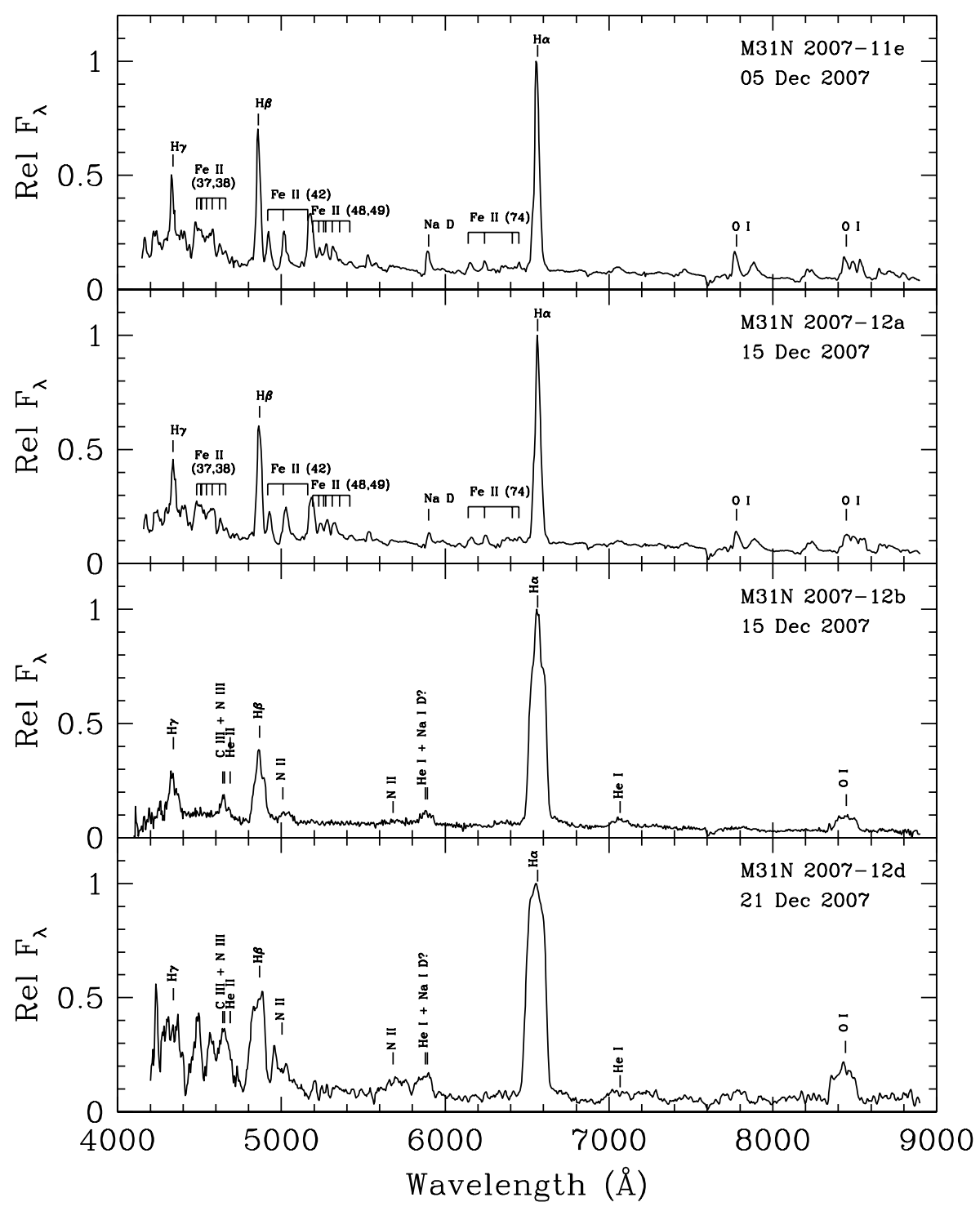

Fig. 9.- Spectra of the M31 novae M31N 2007-11e, 2007-12a, 2007-12b, and 2007-12d, taken 8, 10, 6, and 4 days post discovery, respectively. M31N 2007-11e and M31N 2007-11a are typical Fe II novae, while M31N 2007-12b and M31N 2007-12d are both examples of $\mathrm{He} / \mathrm{N}$ novae. 


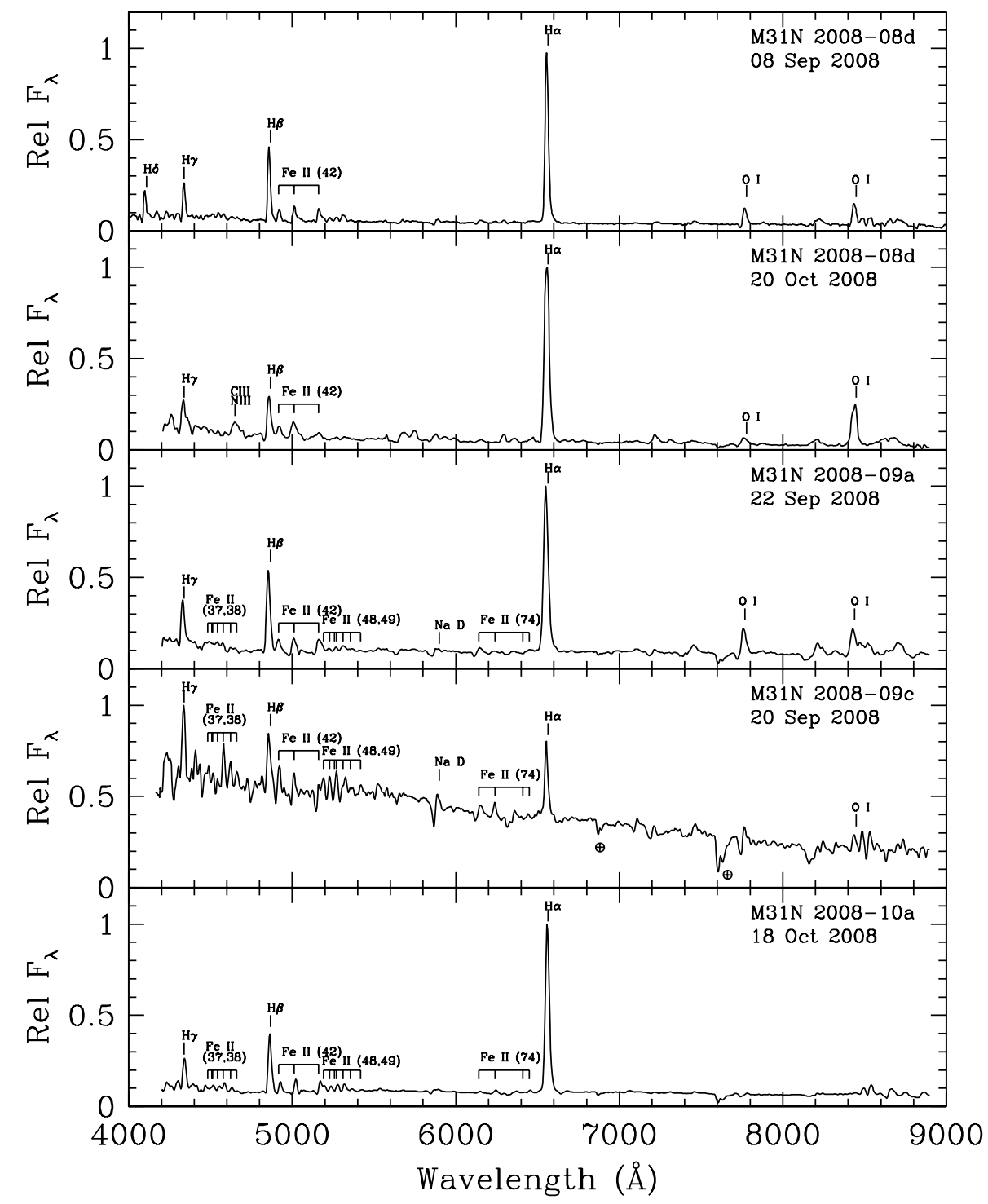

Fig. 10.- Spectra of the M31 novae M31N 2008-08d (two spectra), 2008-09a, 2008-09c, and 2008-10a, taken 14, 57, 10, 6, and 11 days post discovery, respectively. All four novae are of the Fe II spectral type. 


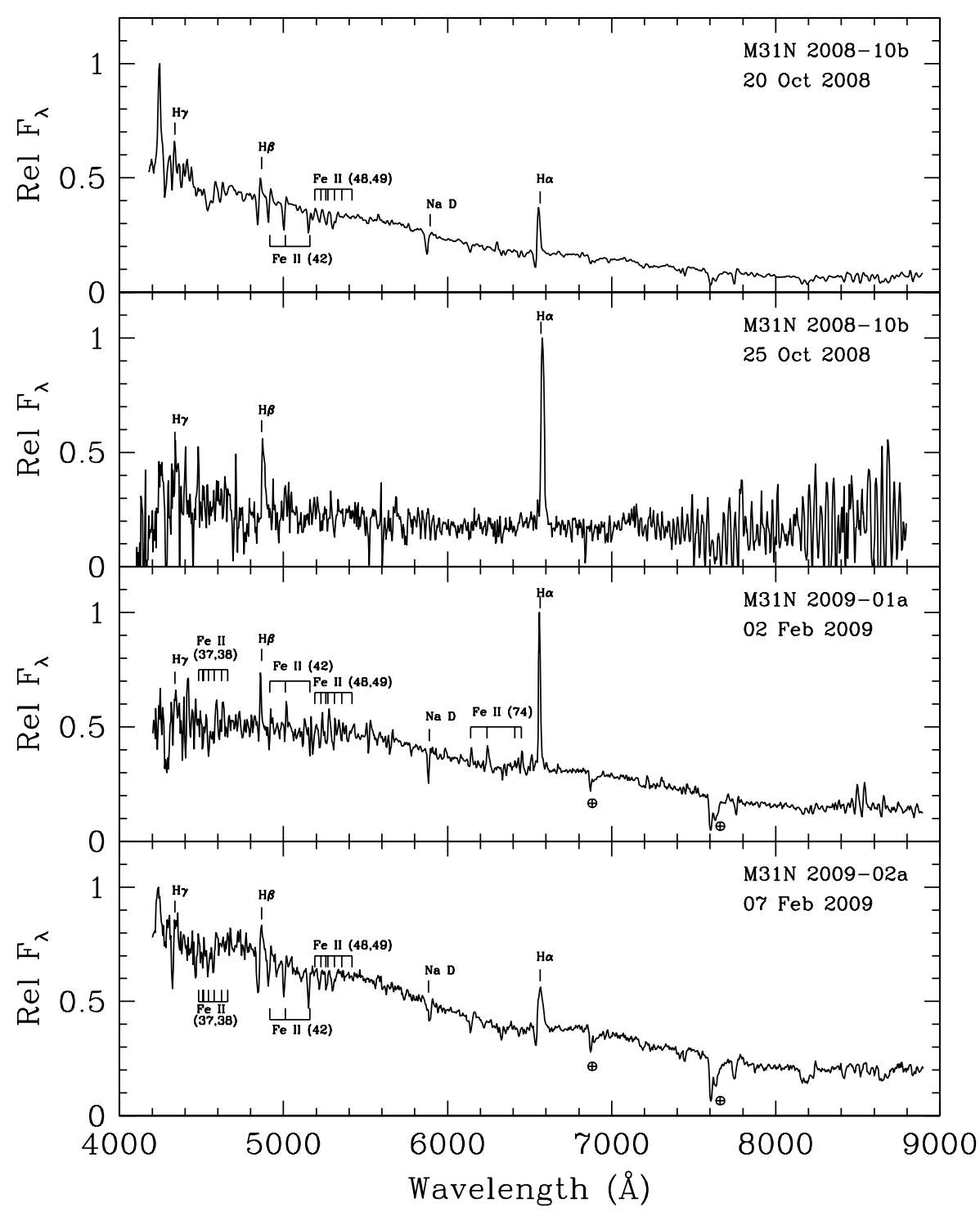

Fig. 11. - Spectra of the M31 novae M31N 2008-10b (two spectra), 2009-01a, and 2009$02 \mathrm{a}$, taken 15, 20, 6, and 2 days, respectively. M31N 2008-10b is a Fe II nova that was observed twice. The first spectrum of M31N 2008-10b and the spectra of and M31N 200901a and M31N 2009-02a display P Cyg profiles indicating that they were observed shortly after eruption. 


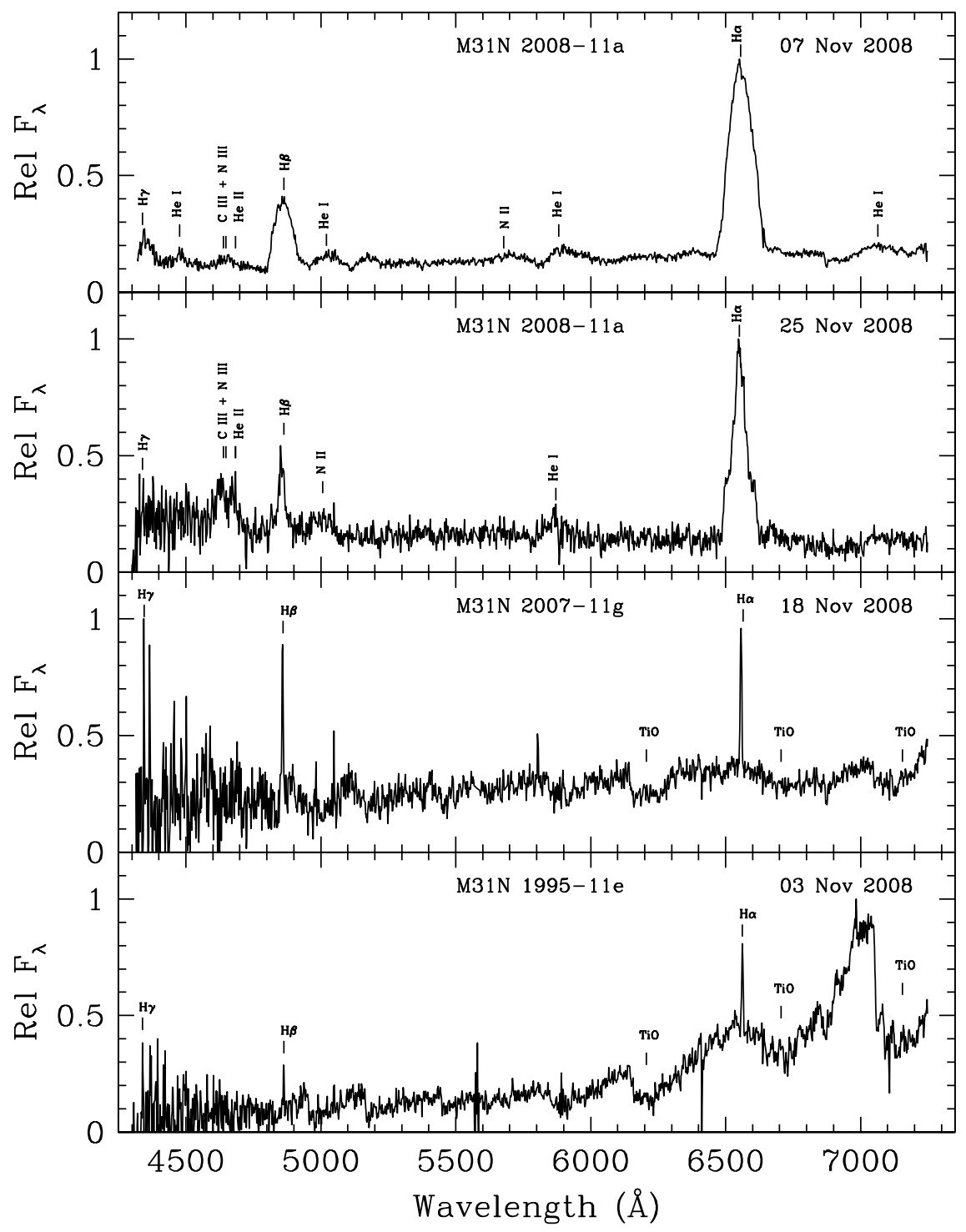

Fig. 12.- Spectra of the M31 nova M31N 2008-11a, a classic He/N nova, taken 4 and 22 days post discovery. The final two objects, M31N 2007-11g and M31N 1995-11e, are examples of long-period variable stars that were mistakenly classified as novae. 

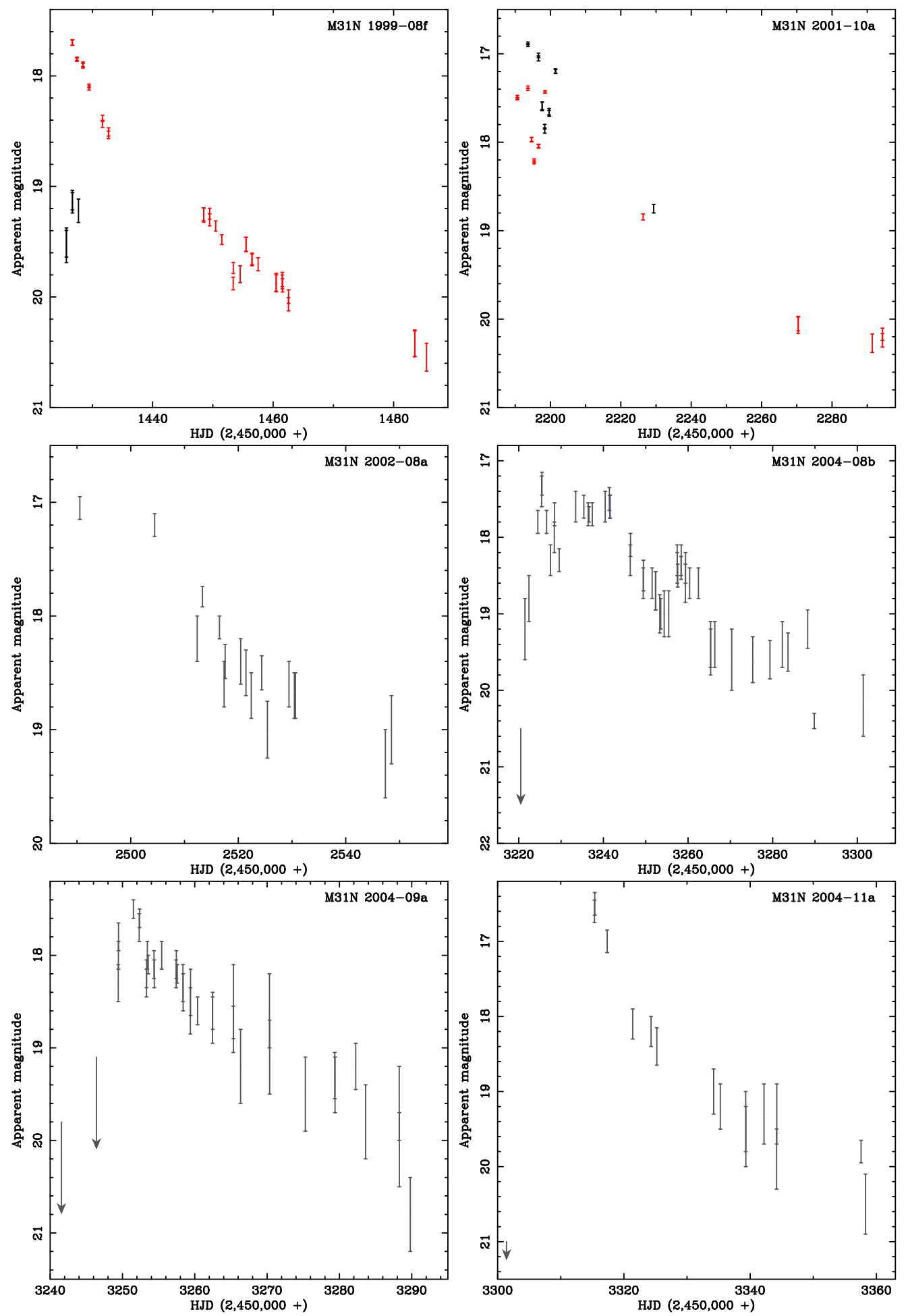

Fig. 13.- Nova light curves. The uncertainties in the photometric measurements are shown as vertical bars with the following colors representing the different bandpasses: $B-$ blue; $V$ - green; $R$ - dark grey; $r^{\prime}$ - red; $i^{\prime}$ - black; $z^{\prime}$ - light grey. Upper flux limits are indicated by downward facing arrows. 

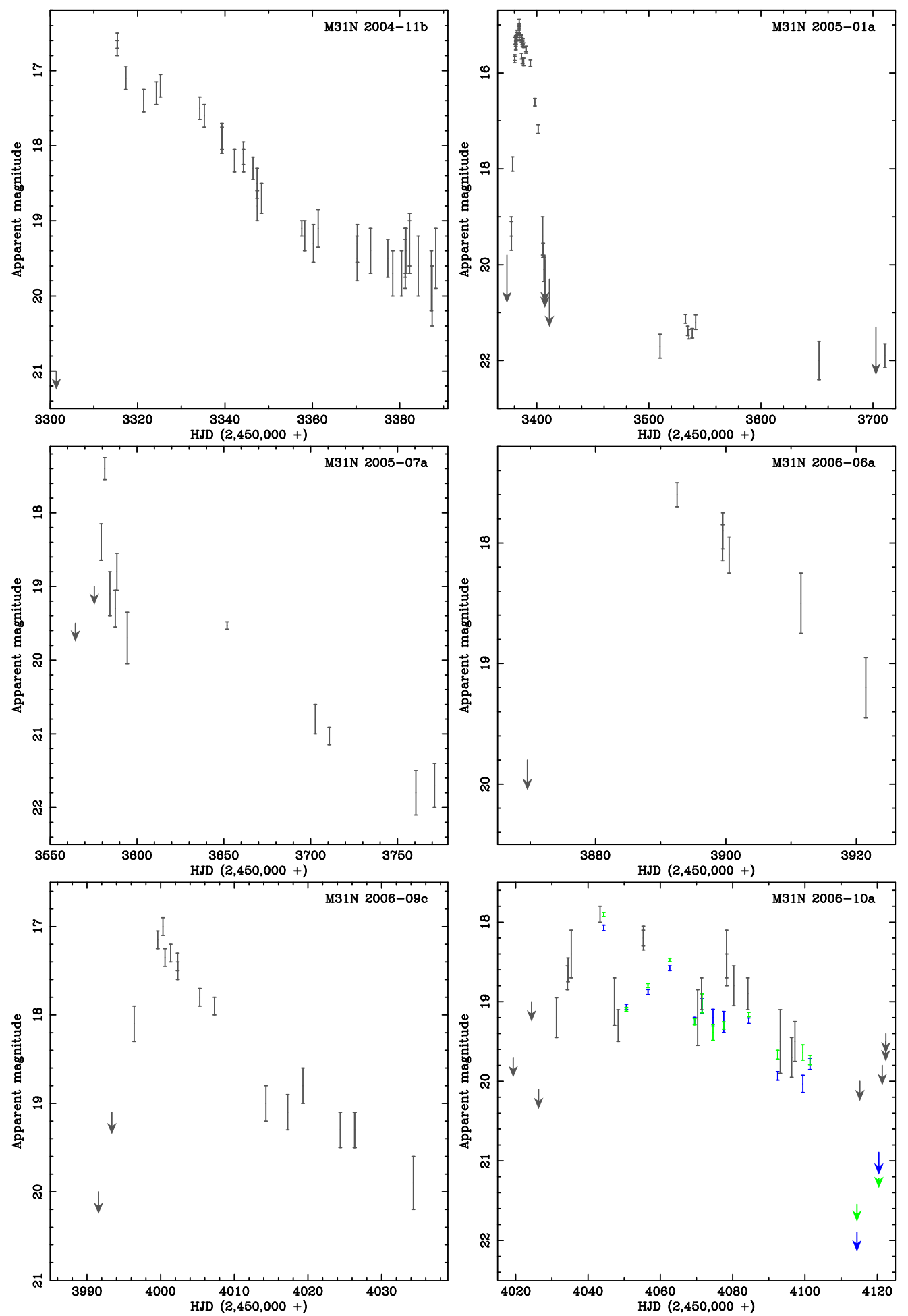

Fig. 14.- Nova light curves (continued). See Fig. 13 for details. 

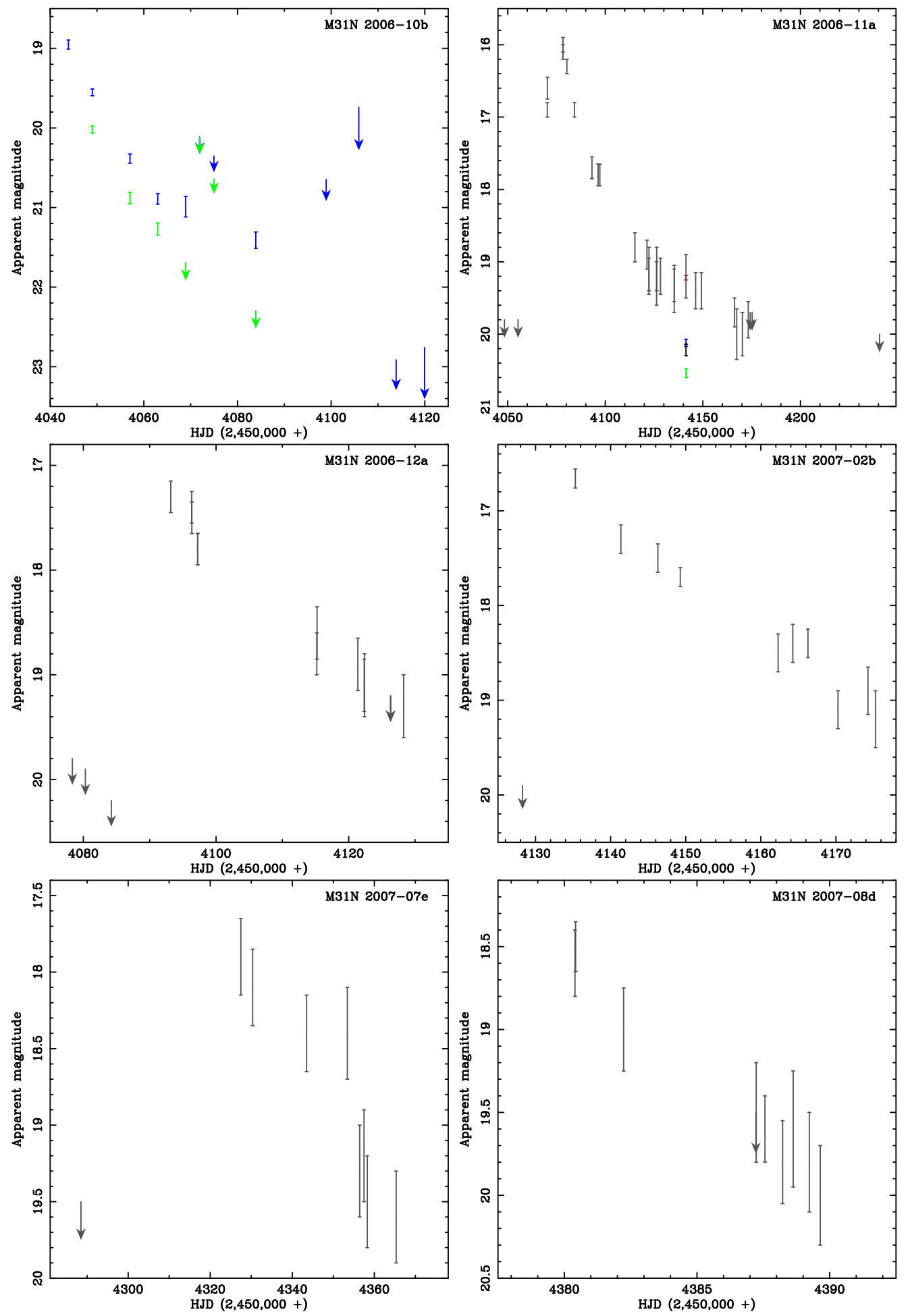

Fig. 15.- Nova light curves (continued). See Fig. 13 for details. 

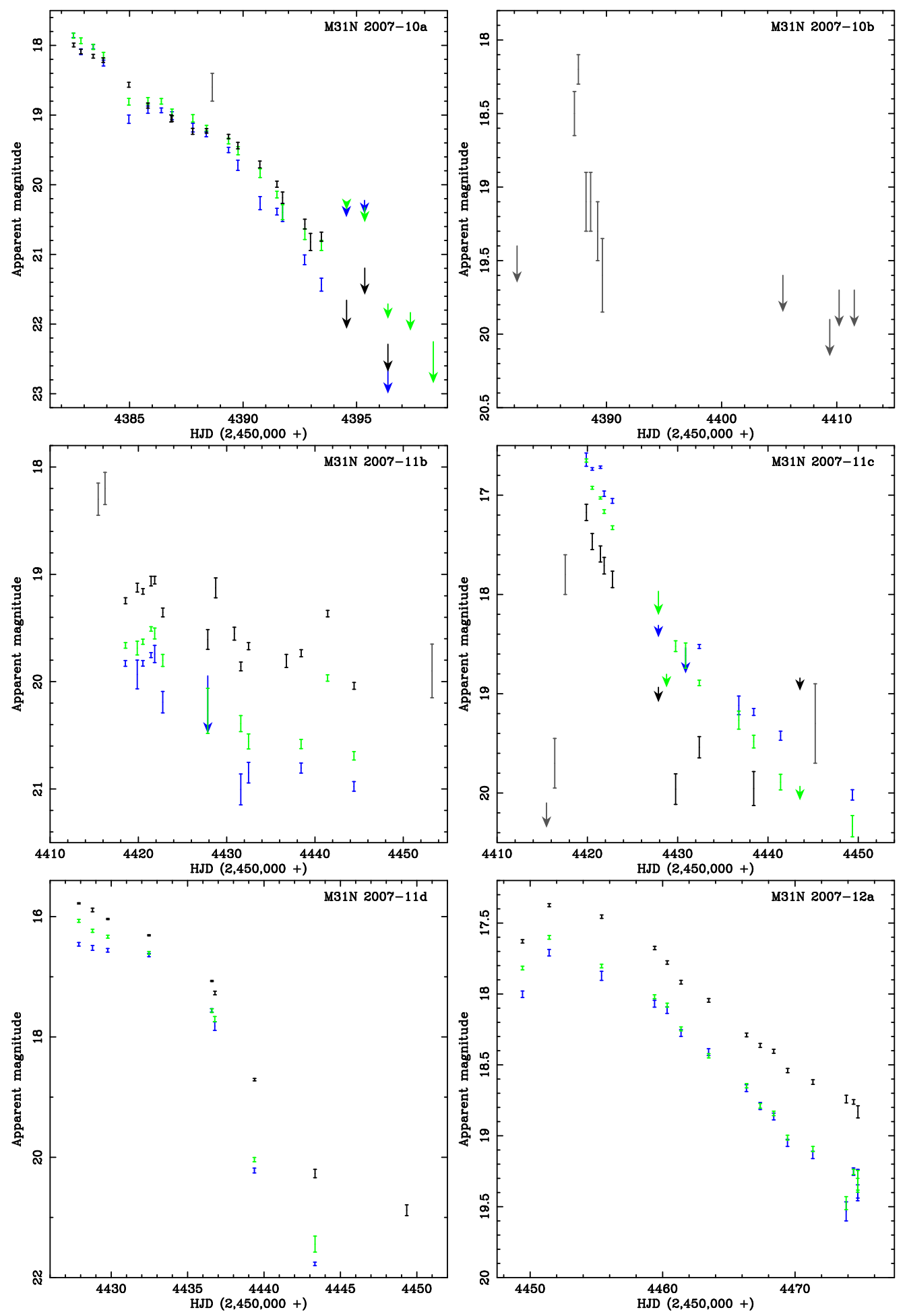

Fig. 16. - Nova light curves (continued). See Fig. 13 for details. 

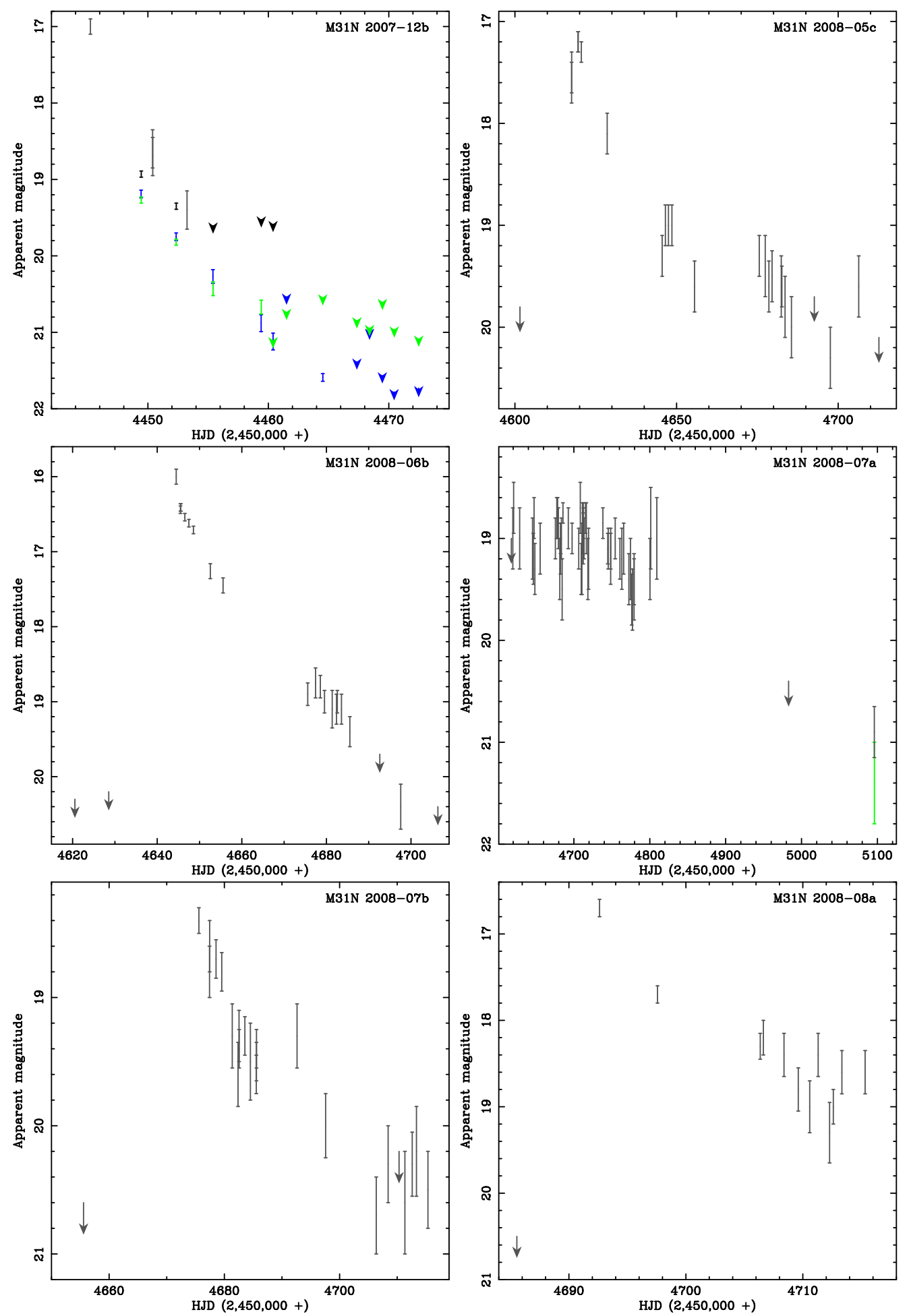

Fig. 17.- Nova light curves (continued). See Fig. 13 for details. 

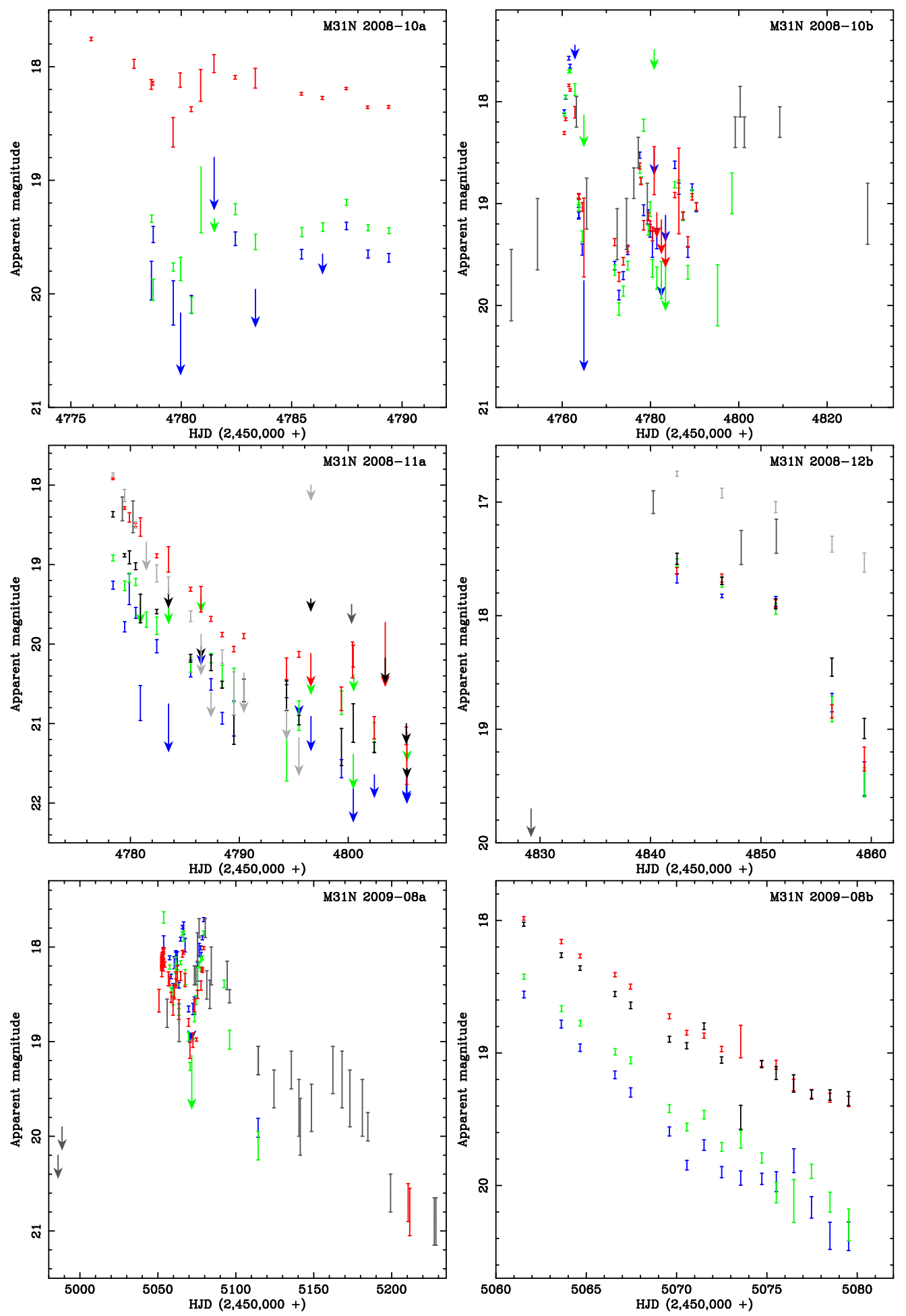

Fig. 18. - Nova light curves (continued). See Fig. 13 for details. 

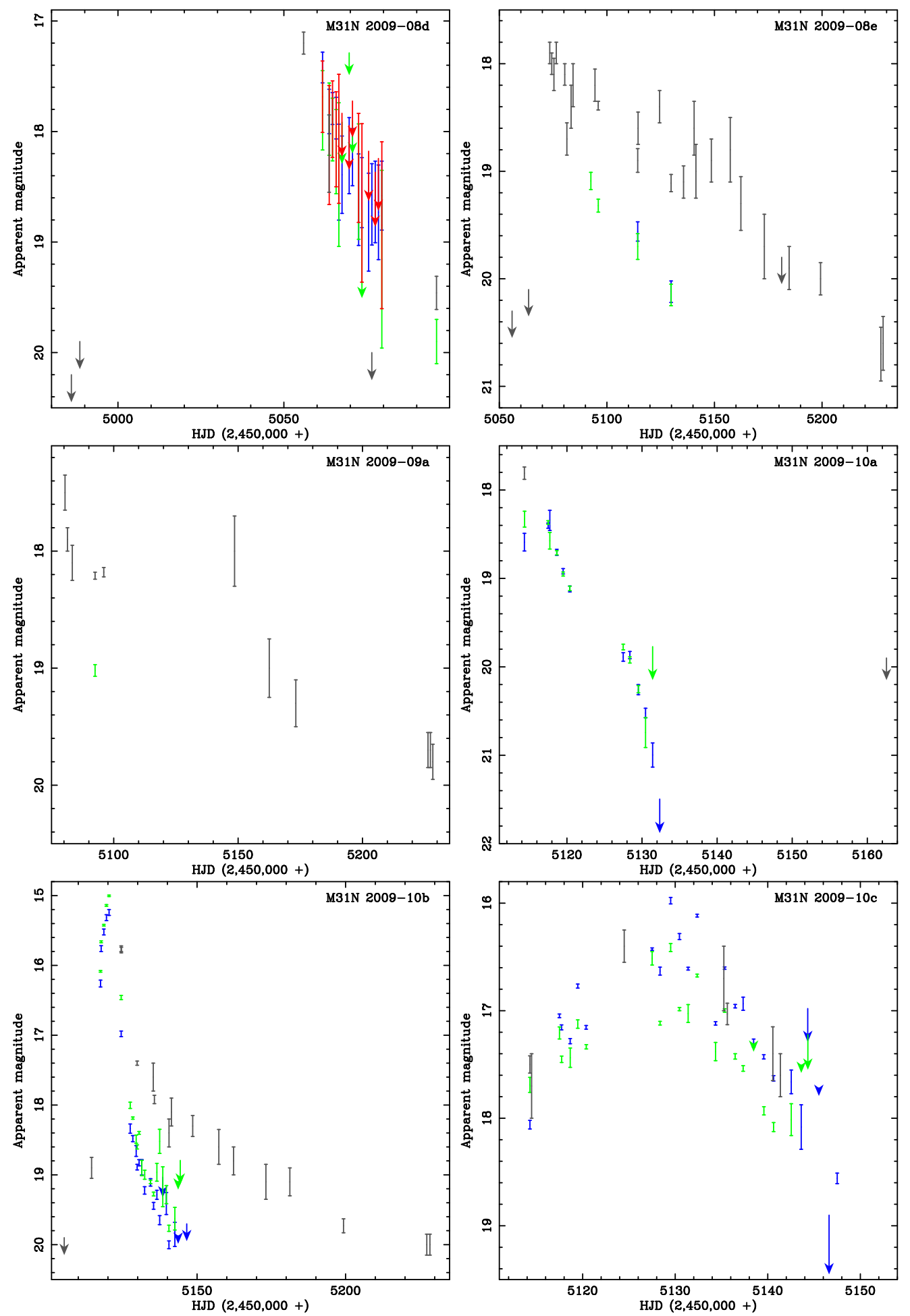

Fig. 19.- Nova light curves (continued). See Fig. 13 for details. 

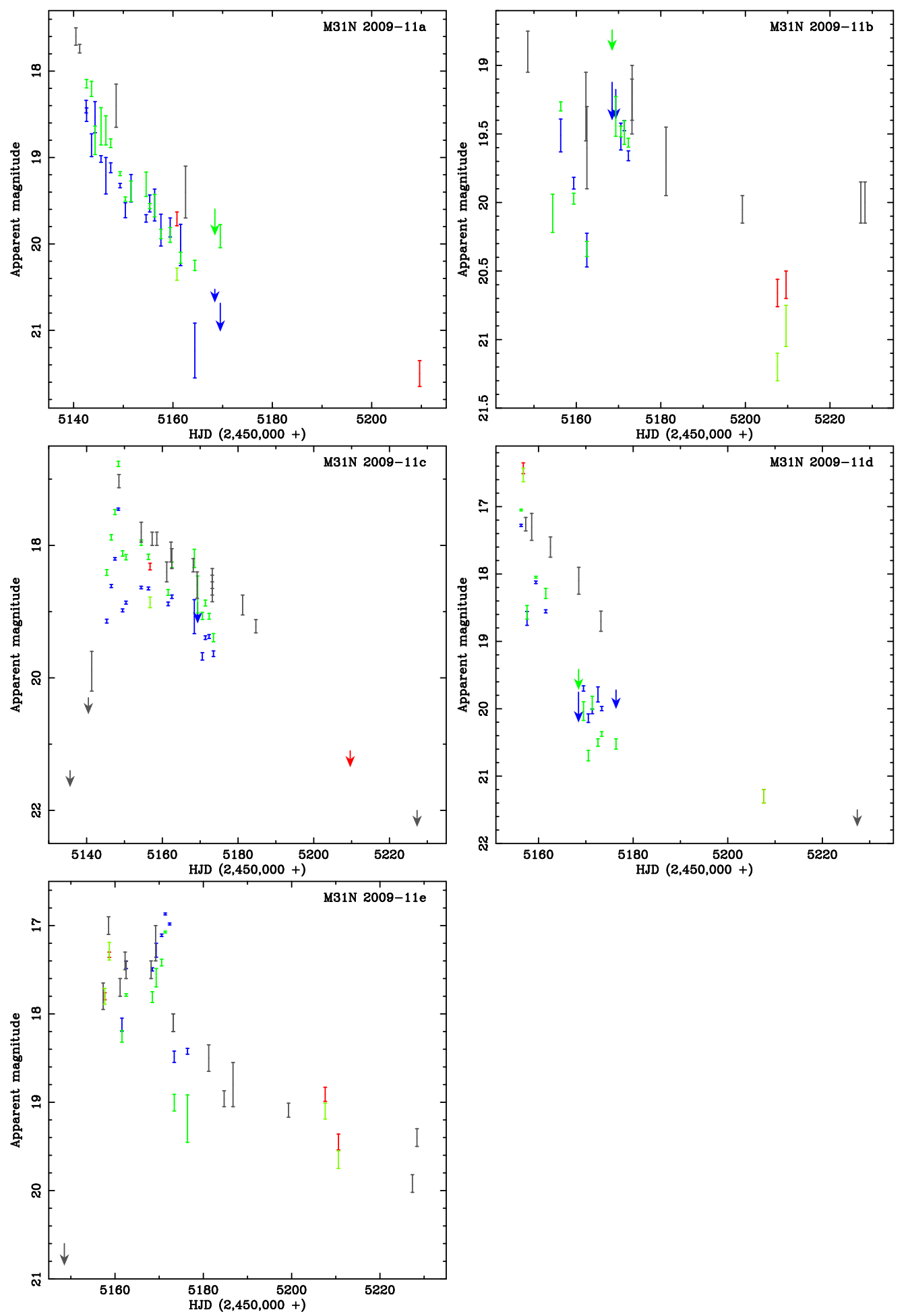

Fig. 20.- Nova light curves (continued). See Fig. 13 for details. 


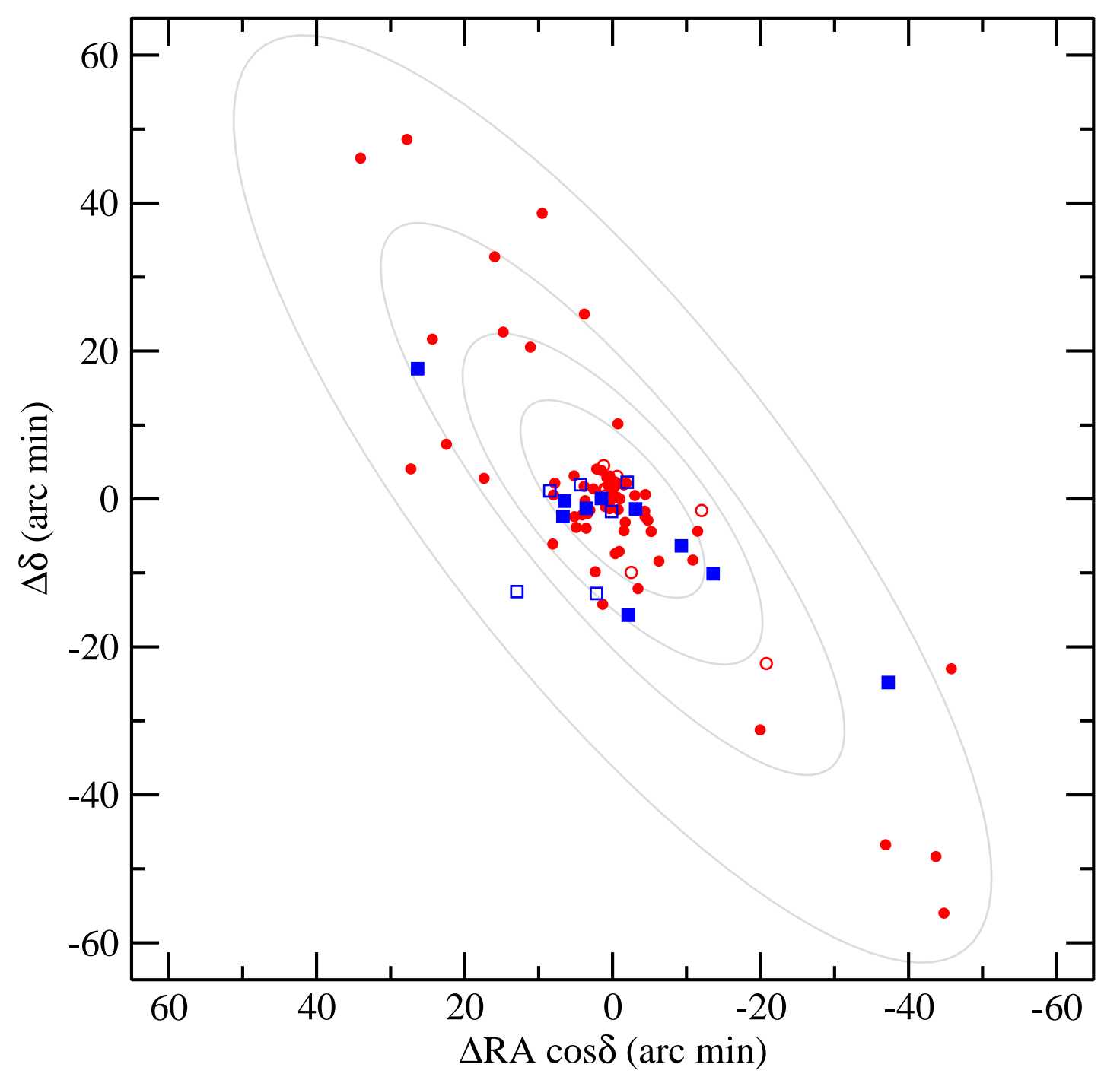

Fig. 21. - The spatial distribution of the 91 M31 novae with known spectroscopic class (see Table 4). The Fe II and Fe II: novae are indicated by filled and open red circles, respectively. The He/N and He/N: novae are represented by filled and open blue squares, respectively. The gray ellipses represent elliptical isophotes from the surface photometry of Kent (1987). 

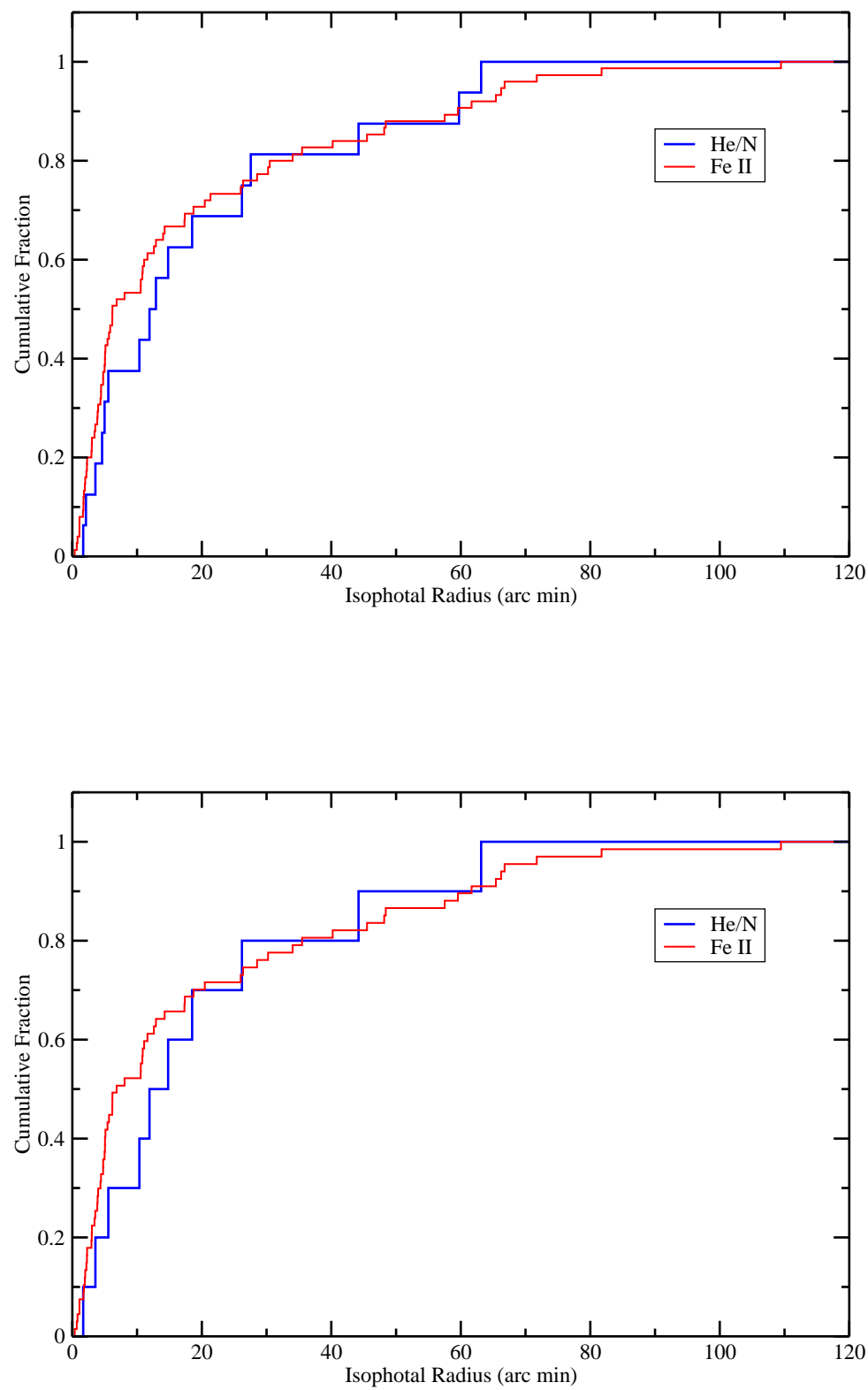

Fig. 22.- The cumulative distributions of Fe II novae compared with that for $\mathrm{He} / \mathrm{N}$ and related novae. The top panel shows the Fe II and Fe II: systems (red) compared with the He/N + hybrid and $\mathrm{He} / \mathrm{N}$ : systems (blue). The bottom panel compares only the well-established Fe II and He/N + hybrid novae. A KS test indicates a $81 \%$ (73\% for bottom panel) probability that the distributions would differ by more than they do if both distributions come from the same parent population. 


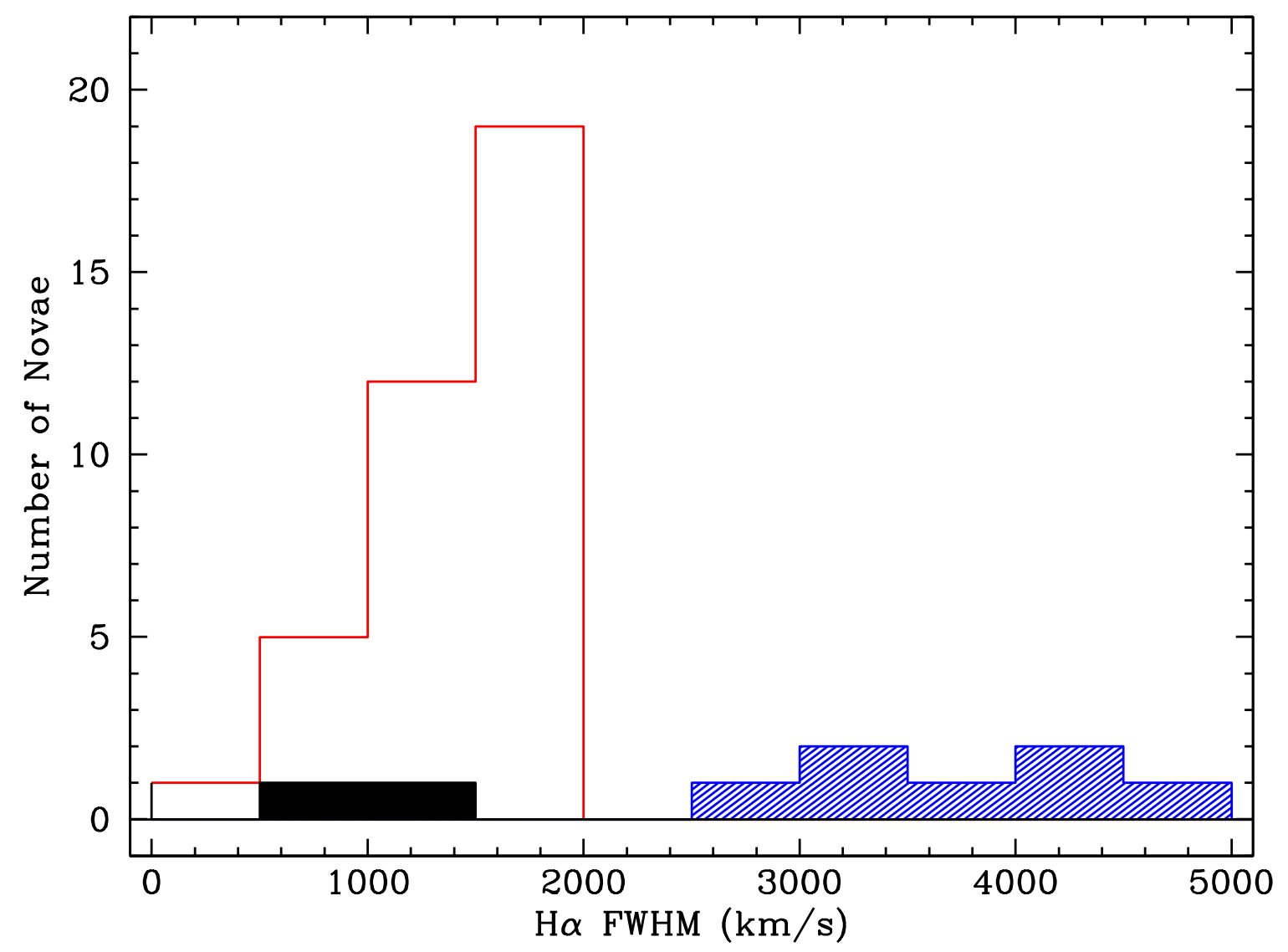

Fig. 23.- The distribution of $\mathrm{H} \alpha$ emission-line FWHM values from the novae in our sample. The novae classified as He/N (cross-hatched blue histogram) are clearly segregated from their Fe II counterparts (red open histogram), with the latter systems having FWHM $\lesssim$ $2500 \mathrm{~km} \mathrm{~s}^{-1}$. Notable exceptions are two peculiar novae classified as $\mathrm{He} / \mathrm{Nn}$ for which we have FWHM measurements that are represented by the filled region. 


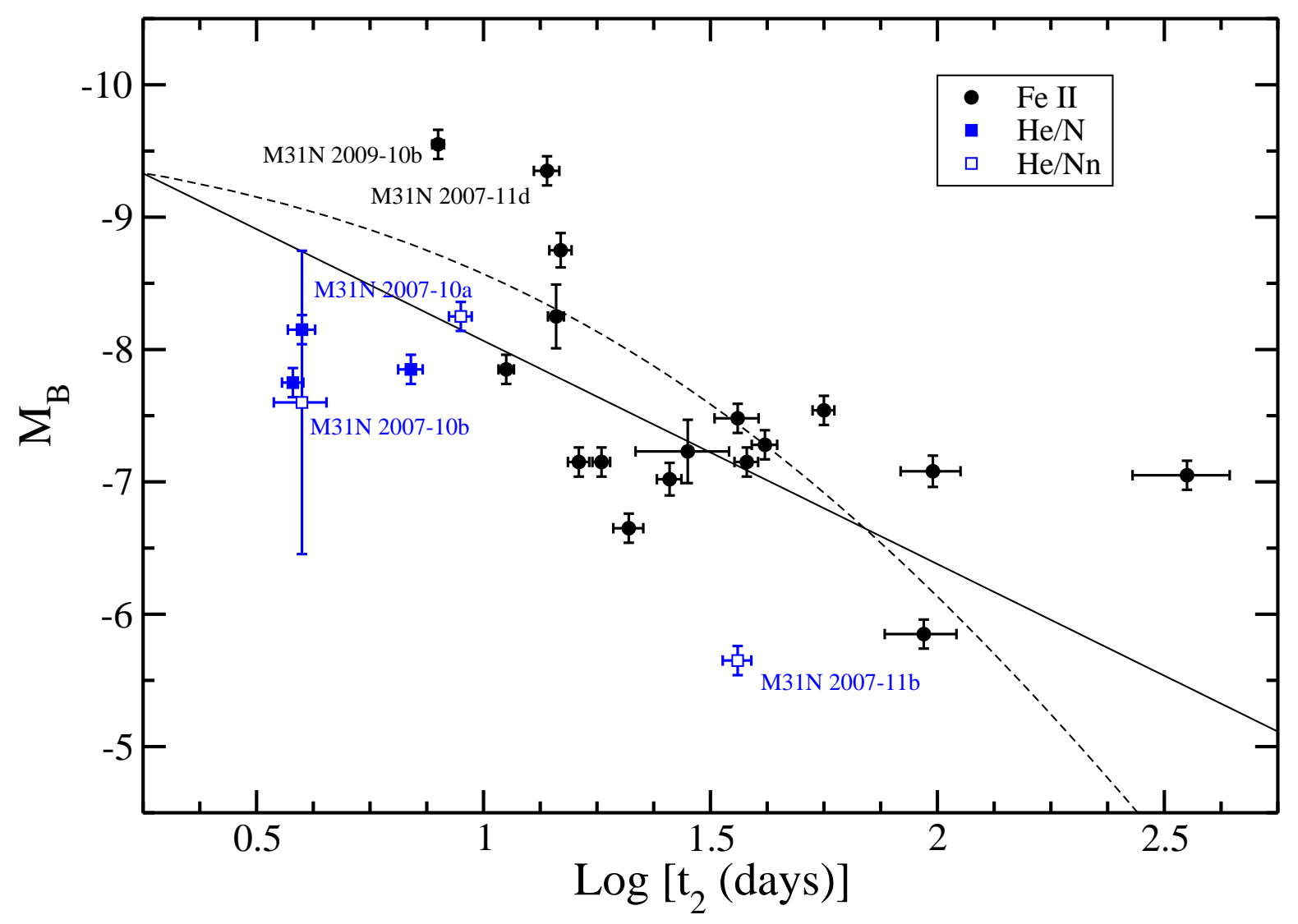

Fig. 24.- The $B$-band maximum-magnitude vs. rate-of-decline relation (MMRD) from our photometric survey. The Fe II, He/N, and $\mathrm{He} / \mathrm{Nn}$ novae are represented by filled red circles, filled blue squares, and open blue squares, respectively. The solid line represents the best-fit relation determined from a weighted linear least-squares analysis (Equation 1), while the dashed line represents the theoretical relation from Livio (1992). Despite the considerable scatter, the data follow the expected trend with the brightest novae generally fading the fastest. 


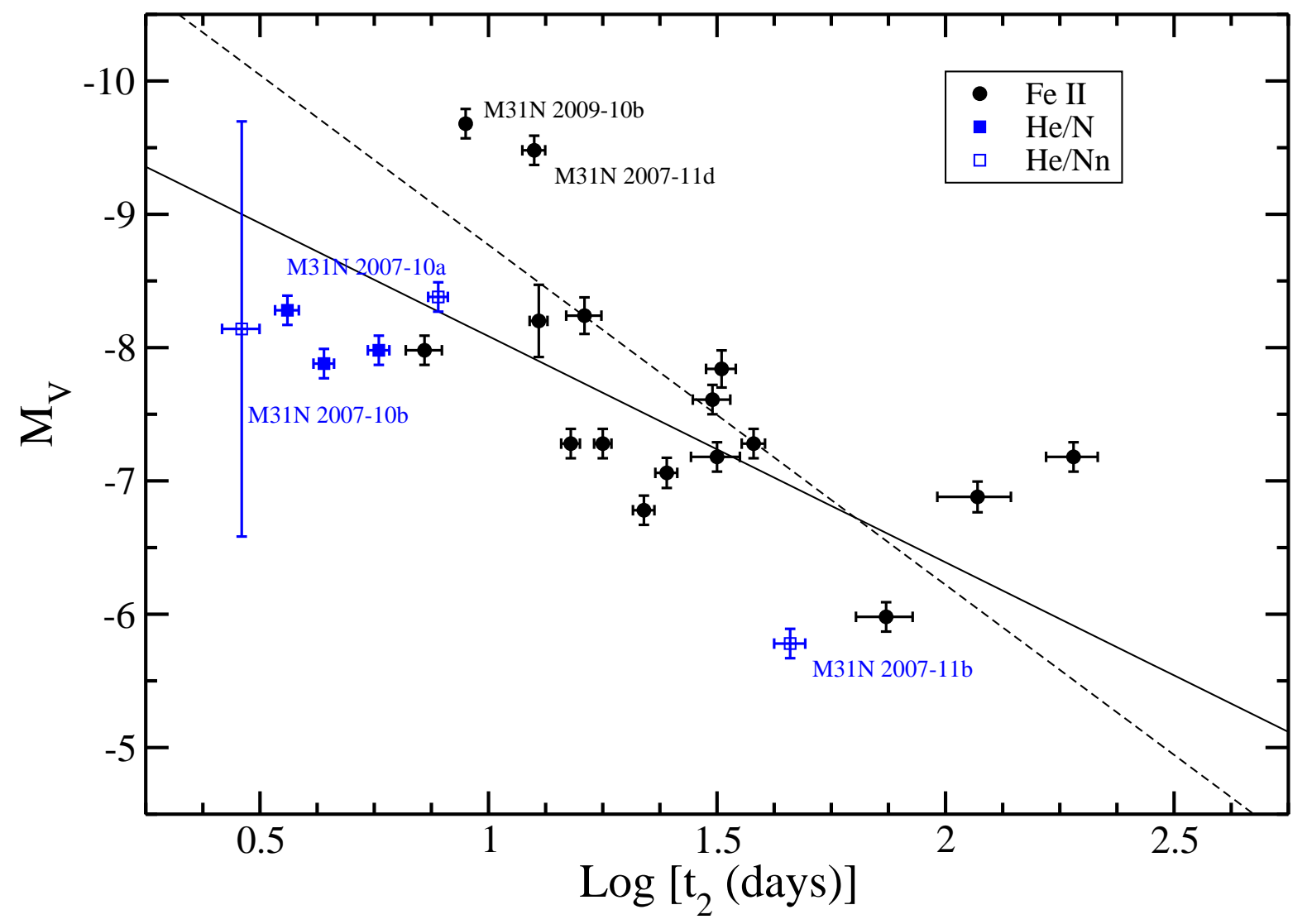

Fig. 25.- The $V$-band MMRD relation from our photometric survey. The symbols have the same meaning as in Fig. 24. The solid line is the best-fit relation given by Equation 2, while the dashed line represents the Galactic $V$-band relation from Downes \& Duerbeck (2000). 


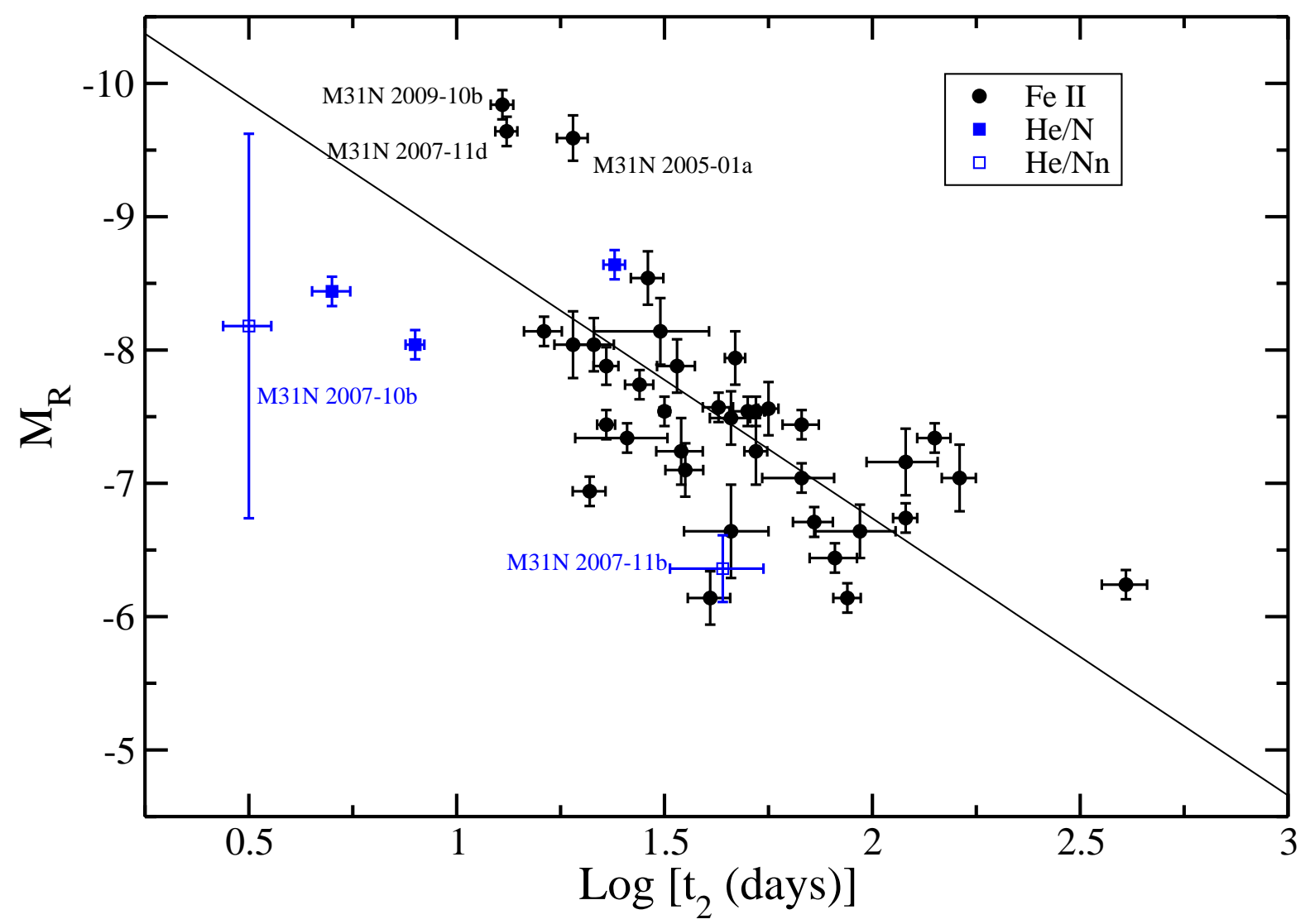

Fig. 26.- The R-band MMRD relation. The symbols have the same meaning as in Fig. 24. The best-fit relation is given by Equation 3. Note the tight group of luminous Fe II novae (M31N 2005-01a, 2007-11d, and 2009-10b) with $M_{V} \lesssim-9$ mag. 


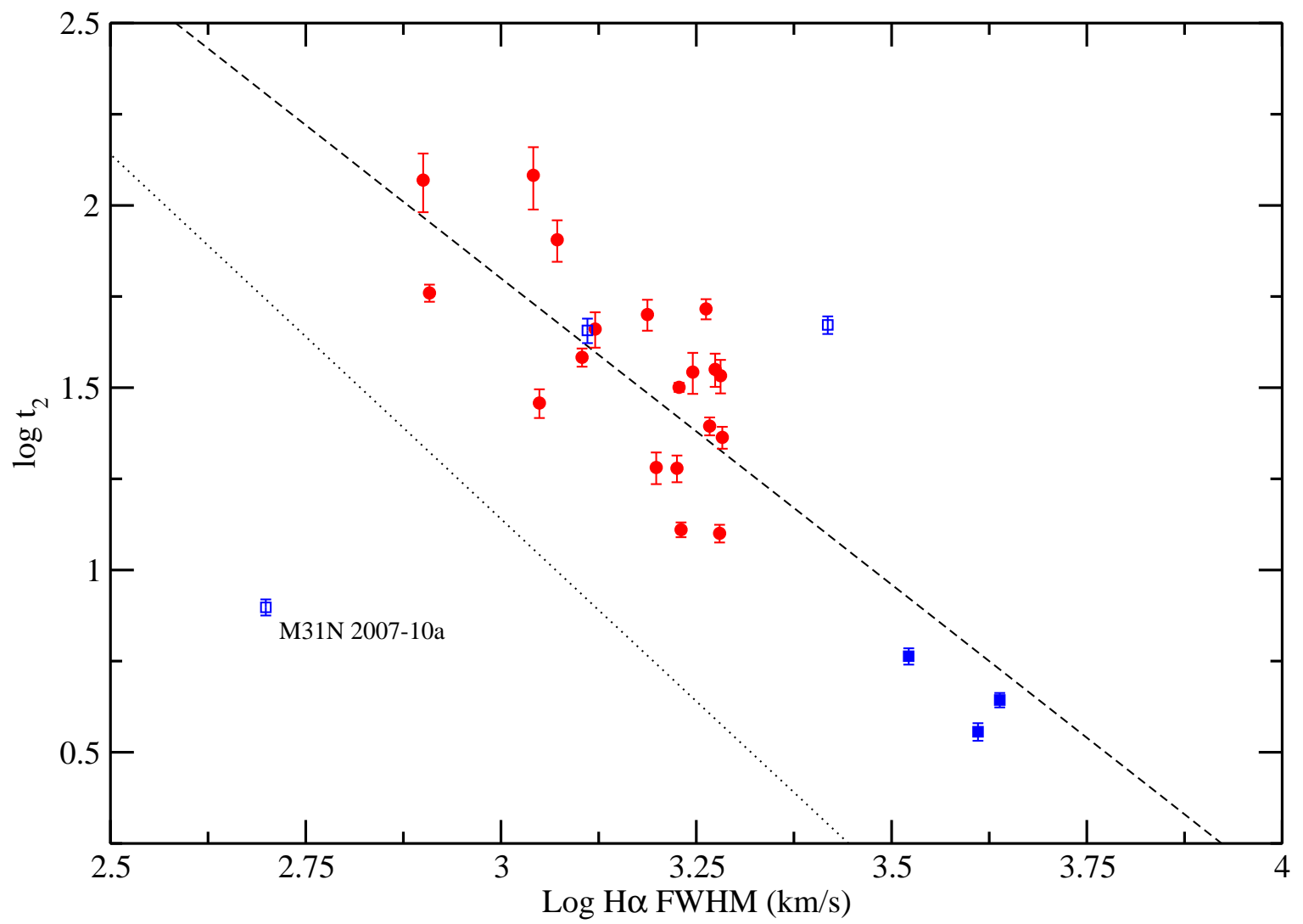

Fig. 27. - The dependence of the light-curve parameter $t_{2}$ on nova expansion velocity (as reflected by the FWHM of $\mathrm{H} \alpha$ ). With the exception of M31N 2007-10a, there is a clear trend of decreasing $t_{2}$ with increasing $\mathrm{H} \alpha$ emission-line width. The red filled circles represent Fe II novae, while the filled (open) blue squares represent $\mathrm{He} / \mathrm{N}(\mathrm{He} / \mathrm{Nn}$ and $\mathrm{He} / \mathrm{N}$ :) novae, respectively. The dashed line reflects the best-fit relation given in Equation 5, while the dotted line gives the Galactic relation of McLaughlin (1960). 


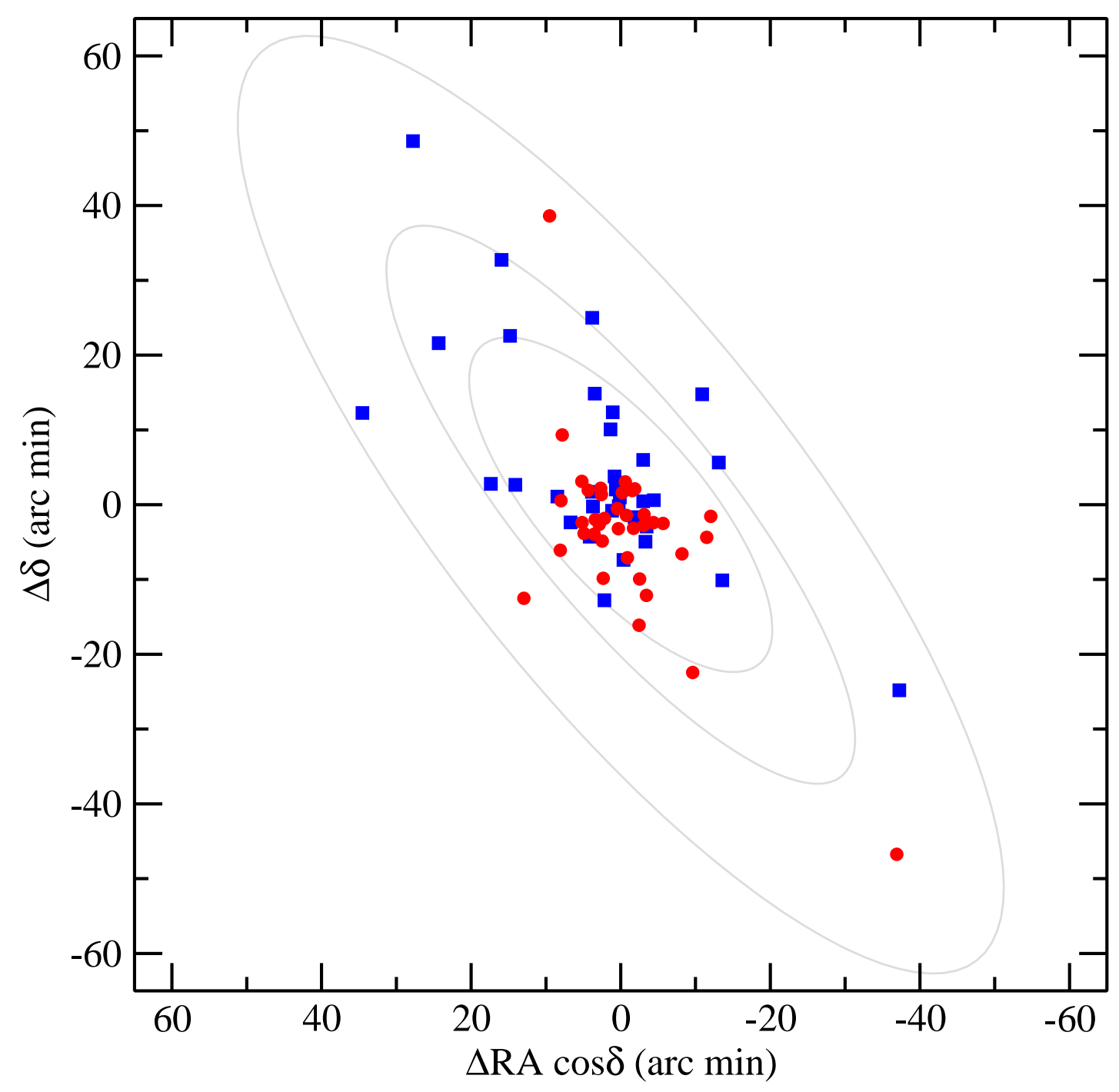

Fig. 28. - The spatial distribution of the 47 M31 novae with measured decline rates from our survey supplemented by 27 decline rates from the "high quality" light-curve sample from the Hubble, Arp, and Rosino surveys Capaccioli et al. (1989). The "very fast" and "fast" novae $\left(t_{2} \leq 25\right.$ days $)$ are indicated by blue squares, with the slower novae $\left(t_{2}>25\right.$ days $)$ are indicated by red circles. The gray ellipses represent elliptical isophotes from the surface photometry of Kent (1987). 


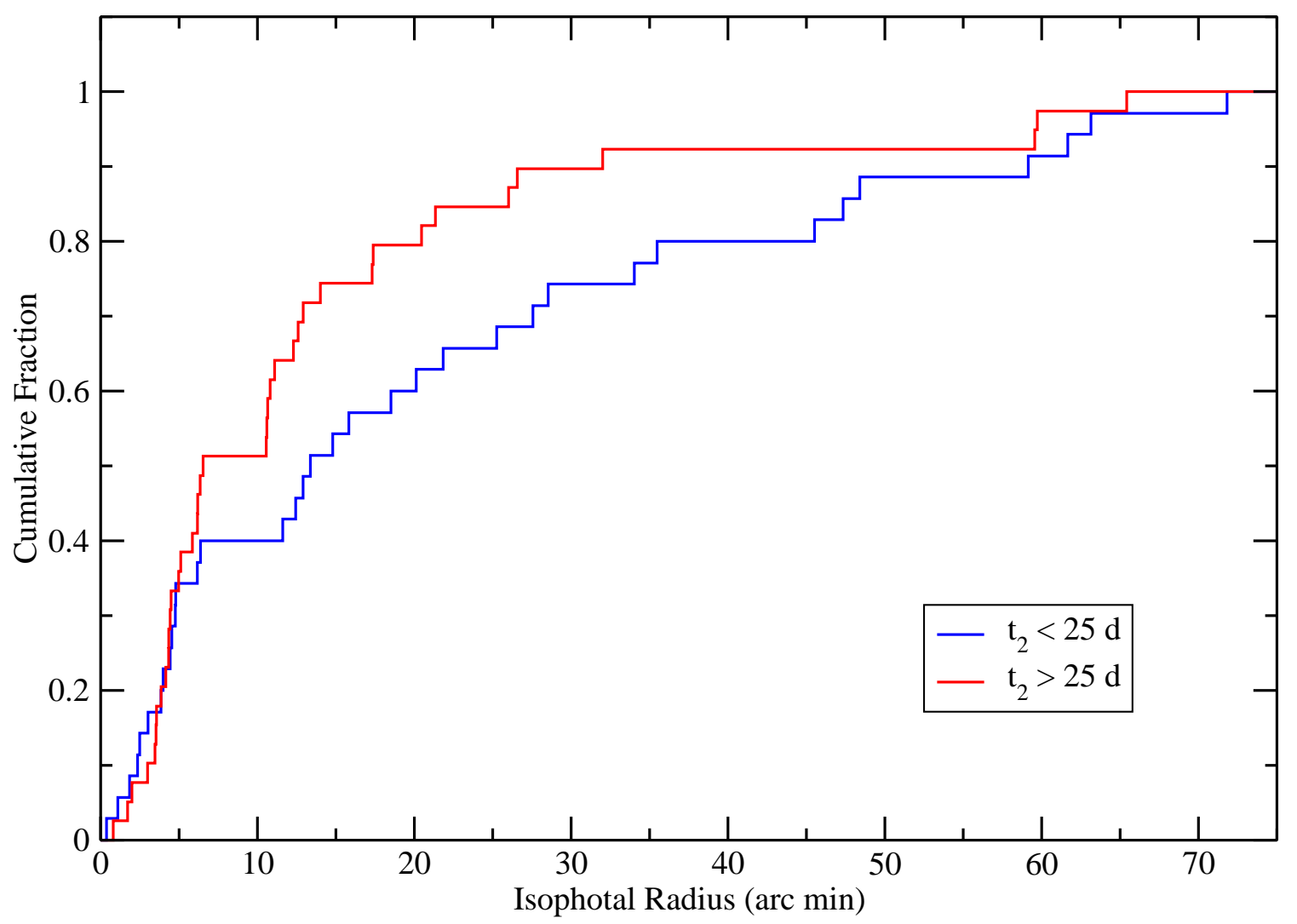

Fig. 29.- The cumulative distributions of the two nova samples from Figure 28. The blue distribution represents "very fast" and "fast" novae ( $t_{2} \leq 25$ days), with the red distribution (broken lines) representing slower novae $\left(t_{2}>25\right.$ days). A KS test indicates a $23 \%$ probability that the distributions would differ by more than they do if both distributions come from the same parent population. Thus, it appears possible that the faster novae are more extended compared with the slower declining systems. 
Table 1. Summary of Lick Spectroscopic Observations ${ }^{\mathrm{a}}$

\begin{tabular}{ccccc}
\hline \hline & $\begin{array}{c}\text { R.A. } \\
\text { Nova }\end{array}$ & $\begin{array}{c}\text { Decl. } \\
(\mathrm{J} 2000.0)\end{array}$ & $\begin{array}{c}\text { Coverage } \\
(\AA)\end{array}$ \\
\hline M31N 1990-10b & $00^{\mathrm{h}} 42^{\mathrm{m}} 36.2^{\mathrm{s}}$ & $41^{\circ} 11^{\prime} 54.0^{\prime \prime}$ & 11 Nov. 1990 & $3900-7100$ \\
M31N 1992-11b & 004236.2 & 411154.0 & 18 Nov. 1992 & $3500-9500$ \\
M31N 1993-06a & 004249.2 & 411727.5 & 28 Jun. 1993 & $4200-7100$ \\
M31N 1993-08a & 004245.1 & 411427.0 & 12 Sep. 1993 & $3500-9500$ \\
M31N 1993-10gb & 004247.7 & 411801.0 & 08 Nov. 1993 & $3500-9500$ \\
M31N 1993-11c ${ }^{\mathrm{b}}$ & 004250.1 & 411728.0 & 17 Nov. 1993 & $4300-7100$ \\
M31N 1998-09d & 004246.6 & 411449.2 & 20 Sep. 1998 & $4300-7000$ \\
M31N 1999-06a & 004249.7 & 411505.6 & 10 Sep. 1999 & $4300-7000$ \\
M31N 1999-08f & 004241.1 & 411912.2 & 17 Sep. 1999 & $4300-7000$ \\
M31N 1999-10a & 004249.7 & 411632.0 & 08 Oct. 1999 & $4300-7000$ \\
M31N 2001-10a & 004303.3 & 411211.5 & 20 Oct. 2001 & $3500-9500$ \\
M31N 2001-12a & 004241.4 & 411624.5 & 11 Feb. 2002 & $3300-7900$ \\
M31N 2002-01b & 004233.9 & 411823.9 & 11 Feb. 2002 & $3300-7900$ \\
M31N 2002-08a & 004230.92 & 410613.1 & 13 Sep. 2002 & $3500-9500$ \\
M31N 2004-08b & 004326.84 & 411640.8 & 10 Sep. 2004 & $3500-9500$ \\
M31N 2004-09a & 004240.27 & 411442.5 & 10 Sep. 2004 & $3500-9500$ \\
M31N 2004-11a & 004242.81 & 411827.9 & 19 Nov. 2004 & $3500-9500$ \\
M31N 2004-11b & 004307.45 & 411804.7 & 19 Nov. 2004 & $3500-9500$ \\
M31N 2005-01a & 004228.39 & 411636.2 & 16 Jan. 2005 & $3500-9500$ \\
M31N 2005-07a & 004250.79 & 412039.8 & 01 Aug. 2005 & $3500-9500$ \\
M31N 2008-08d & 004548.25 & 430222.2 & 08 Sep. 2008 & $3500-9500$ \\
\hline
\end{tabular}

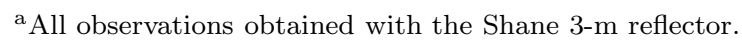

${ }^{\mathrm{b}}$ Due to ambiguity in the data logs from November 1993, it is possible that the dates of observation (and thus the spectra) for these two novae are reversed. 
Table 2. Summary of HET Spectroscopic Observations

\begin{tabular}{|c|c|c|c|c|c|c|}
\hline Nova & $\begin{array}{c}\text { R.A. } \\
\text { (J2000.0) }\end{array}$ & $\begin{array}{c}\text { Decl. } \\
(\mathrm{J} 2000.0)\end{array}$ & UT Date & $\begin{array}{l}\text { Exp. } \\
\text { (sec) }\end{array}$ & $\begin{array}{c}\text { Coverage } \\
(\AA)\end{array}$ & Weather \\
\hline M31N 1995-11e & $00^{\mathrm{h}} 45^{\mathrm{m}} 09.9^{\mathrm{s}}$ & $41^{\circ} 52^{\prime} 03.0^{\prime \prime}$ & 03.30 Nov. 2008 & 1200 & $4275-7250$ & Spec \\
\hline M31N 2006-09c & 004242.38 & 410845.5 & 24.18 Sep. 2006 & 1800 & $4275-7250$ & Spec \\
\hline M31N 2006-10a & 004143.23 & 411145.9 & 30.31 Oct. 2006 & 1500 & $4275-7250$ & Spec \\
\hline \multirow[t]{2}{*}{ M31N 2006-10b } & 003927.38 & 405109.8 & 01.09 Nov. 2006 & 1800 & $4275-7250$ & Spec \\
\hline & & & 23.24 Nov. 2006 & 1200 & $4275-7250$ & Phot \\
\hline M31N 2006-11a & 004256.81 & 410618.4 & 28.23 Nov. 2006 & 1200 & $4275-7250$ & Spec \\
\hline M31N 2006-12a & 004221.09 & 411345.3 & 08.13 Jan. 2007 & 1200 & $4275-7250$ & Spec \\
\hline M31N 2006-12b & 004211.14 & 410743.8 & 10.11 Jan. 2007 & 3200 & $4275-7250$ & Cldy \\
\hline M31N 2007-02a & 004059.02 & 404452.7 & 09.07 Feb. 2007 & 900 & $4275-7250$ & Spec \\
\hline M31N 2007-02b & 004140.32 & 411433.5 & 10.06 Feb. 2007 & 900 & $4275-7250$ & Phot \\
\hline M31N 2007-06b & 004233.14 & 410025.9 & 25.34 Jul. 2007 & 3600 & $4275-7250$ & Spec \\
\hline M31N 2007-08d & 003930.27 & 402914.2 & 13.44 Sep. 2007 & 1200 & $4275-7250$ & Spec \\
\hline M31N 2007-10a & 004255.95 & 410322.0 & 19.13 Oct. 2007 & 1400 & $4275-7250$ & Phot \\
\hline M31N 2007-11b & 004352.99 & 410336.2 & 11.30 Nov. 2007 & 1200 & $4100-8900$ & Spec \\
\hline M31N 2007-11c & 004304.14 & 411554.3 & 16.28 Nov. 2007 & 1200 & $4100-8900$ & Spec \\
\hline \multirow{2}{*}{ M31N 2007-11d } & 004454.60 & 413740.0 & 21.27 Nov. 2007 & 600 & $4100-8900$ & Phot \\
\hline & & & 04.22 Dec. 2007 & 1200 & $4100-8900$ & Phot \\
\hline M31N 2007-11e & 004547.74 & 420203.5 & 05.23 Dec. 2007 & 1200 & $4100-8900$ & Phot \\
\hline M31N 2007-11g & 004415.80 & 411350.3 & 18.28 Nov. 2008 & 1200 & $4275-7250$ & Spec \\
\hline M31N 2007-12a & 004403.51 & 413841.1 & 15.20 Dec. 2007 & 1200 & $4100-8900$ & Phot \\
\hline M31N 2007-12b & 004319.94 & 411346.6 & 15.18 Dec. 2007 & 1000 & $4100-8900$ & Phot \\
\hline M31N 2007-12d & 004154.96 & 410947.3 & 21.17 Dec. 2007 & 1800 & $4100-8900$ & Spec \\
\hline M31N 2008-08d & 004548.25 & $4302 \quad 22.2$ & 20.13 Oct. 2008 & 1200 & $4100-8900$ & Phot \\
\hline M31N 2008-09a & 004146.72 & 410752.1 & 22.41 Sep. 2008 & 1200 & $4100-8900$ & Spec \\
\hline M31N 2008-09c & 004251.42 & 410154.0 & 20.18 Sep. 2008 & 1200 & $4100-8900$ & Spec \\
\hline M31N 2008-10a & 004335.46 & 415444.2 & 18.34 Oct. 2008 & 1200 & $4100-8900$ & Phot \\
\hline \multirow[t]{2}{*}{ M31N 2008-10b } & 004302.42 & 411409.9 & 20.11 Oct. 2008 & 1200 & $4100-8900$ & Phot \\
\hline & & & 25.33 Oct. 2008 & 1200 & $4100-8900$ & Spec \\
\hline \multirow[t]{2}{*}{ M31N 2008-11a } & 004132.26 & 410601.0 & 07.29 Nov. 2008 & 1200 & $4275-7250$ & Phot \\
\hline & & & 25.25 Nov. 2008 & 1800 & $4275-7250$ & Spec \\
\hline M31N 2009-01a & 004444.03 & 412328.3 & 02.07 Feb. 2009 & 1350 & $4100-8900$ & Phot \\
\hline M31N 2009-02a & 004343.81 & 413638.8 & 07.07 Feb. 2009 & 900 & $4100-8900$ & Spec \\
\hline
\end{tabular}


Table 3. Photometric Observations

\begin{tabular}{cccc}
\hline \hline JD & & & \\
$(2,450,000+)$ & Mag & Filter & Notes $^{\mathrm{a}}$ \\
\hline & & & \\
\hline & M31N $1999-08 \mathrm{f}$ & & \\
\hline 1427.686 & $17.700 \pm 0.024$ & $r^{\prime}$ & $(29)$ \\
1427.690 & $17.697 \pm 0.025$ & $r^{\prime}$ & $(29)$ \\
1428.444 & $17.853 \pm 0.016$ & $r^{\prime}$ & $(29)$ \\
1428.452 & $17.845 \pm 0.015$ & $r^{\prime}$ & $(29)$ \\
1429.452 & $17.895 \pm 0.021$ & $r^{\prime}$ & $(29)$ \\
1429.456 & $17.908 \pm 0.021$ & $r^{\prime}$ & $(29)$ \\
1430.467 & $18.090 \pm 0.016$ & $r^{\prime}$ & $(29)$ \\
1430.471 & $18.109 \pm 0.019$ & $r^{\prime}$ & $(29)$ \\
1432.686 & $18.384 \pm 0.029$ & $r^{\prime}$ & $(29)$ \\
1432.690 & $18.434 \pm 0.032$ & $r^{\prime}$ & $(29)$ \\
1433.678 & $18.534 \pm 0.034$ & $r^{\prime}$ & $(29)$ \\
1433.686 & $18.507 \pm 0.037$ & $r^{\prime}$ & $(29)$ \\
1449.467 & $19.258 \pm 0.065$ & $r^{\prime}$ & $(29)$ \\
1449.471 & $19.253 \pm 0.057$ & $r^{\prime}$ & $(29)$ \\
1450.463 & $19.302 \pm 0.054$ & $r^{\prime}$ & $(29)$ \\
1450.467 & $19.247 \pm 0.049$ & $r^{\prime}$ & $(29)$ \\
1451.475 & $19.358 \pm 0.046$ & $r^{\prime}$ & $(29)$ \\
1452.491 & $19.481 \pm 0.044$ & $r^{\prime}$ & $(29)$ \\
1454.374 & $19.878 \pm 0.057$ & $r^{\prime}$ & $(29)$ \\
1454.389 & $19.738 \pm 0.049$ & $r^{\prime}$ & $(29)$ \\
1455.538 & $19.794 \pm 0.076$ & $r^{\prime}$ & $(29)$ \\
1456.495 & $19.523 \pm 0.063$ & $r^{\prime}$ & $(29)$ \\
1456.499 & $19.525 \pm 0.065$ & $r^{\prime}$ & $(29)$ \\
1457.495 & $19.664 \pm 0.053$ & $r^{\prime}$ & $(29)$ \\
1457.499 & $19.656 \pm 0.052$ & $r^{\prime}$ & $(29)$ \\
1458.506 & $19.704 \pm 0.059$ & $r^{\prime}$ & $(29)$ \\
1461.510 & $19.877 \pm 0.076$ & $r^{\prime}$ & $(29)$ \\
1461.514 & $19.867 \pm 0.081$ & $r^{\prime}$ & $(29)$ \\
1462.530 & $19.864 \pm 0.063$ & $r^{\prime}$ & $(29)$ \\
1462.534 & $19.842 \pm 0.065$ & $r^{\prime}$ & $(29)$ \\
1462.561 & $19.895 \pm 0.060$ & $r^{\prime}$ & $(29)$ \\
1463.549 & $20.066 \pm 0.060$ & $r^{\prime}$ & $(29)$ \\
1463.557 & $19.996 \pm 0.061$ & $r^{\prime}$ & $(29)$ \\
1484.499 & $20.424 \pm 0.117$ & $r^{\prime}$ & $(29)$ \\
1484.502 & $20.418 \pm 0.120$ & $r^{\prime}$ & $(29)$ \\
1486.456 & $20.545 \pm 0.126$ & $r^{\prime}$ & $(29)$ \\
& & & \\
1426.694 & $19.544 \pm 0.146$ & $i^{\prime}$ & $(29)$ \\
& $19.507 \pm 0.132$ & $i^{\prime}$ & $(29)$ \\
& $19.124 \pm 0.089$ & $i^{\prime}$ & $(29)$ \\
& $19.148 \pm 0.092$ & $i^{\prime}$ & $(29)$ \\
& & & $(29)$
\end{tabular}


Table 3-Continued

\begin{tabular}{|c|c|c|c|}
\hline $\begin{array}{c}\mathrm{JD} \\
(2,450,000+)\end{array}$ & Mag & Filter & Notes $^{\mathrm{a}}$ \\
\hline \multicolumn{4}{|c|}{ M31N 2001-10a } \\
\hline 2191.616 & $17.512 \pm 0.008$ & $r^{\prime}$ & $(29)$ \\
\hline 2191.620 & $17.481 \pm 0.011$ & $r^{\prime}$ & $(29)$ \\
\hline 2194.620 & $17.378 \pm 0.014$ & $r^{\prime}$ & $(29)$ \\
\hline 2194.624 & $17.402 \pm 0.013$ & $r^{\prime}$ & (29) \\
\hline 2195.640 & $17.968 \pm 0.021$ & $r^{\prime}$ & (29) \\
\hline 2195.643 & $17.971 \pm 0.026$ & $r^{\prime}$ & $(29)$ \\
\hline 2196.382 & $18.227 \pm 0.017$ & $r^{\prime}$ & $(29)$ \\
\hline 2196.386 & $18.207 \pm 0.018$ & $r^{\prime}$ & (29) \\
\hline 2197.628 & $18.043 \pm 0.020$ & $r^{\prime}$ & (29) \\
\hline 2197.632 & $18.047 \pm 0.020$ & $r^{\prime}$ & (29) \\
\hline 2199.452 & $17.430 \pm 0.013$ & $r^{\prime}$ & (29) \\
\hline 2199.456 & $17.433 \pm 0.013$ & $r^{\prime}$ & (29) \\
\hline 2227.339 & $18.845 \pm 0.034$ & $r^{\prime}$ & $(29)$ \\
\hline 2271.405 & $20.056 \pm 0.078$ & $r^{\prime}$ & $(29)$ \\
\hline 2271.409 & $20.065 \pm 0.096$ & $r^{\prime}$ & $(29)$ \\
\hline 2292.429 & $20.273 \pm 0.105$ & $r^{\prime}$ & (29) \\
\hline 2295.343 & $20.171 \pm 0.068$ & $r^{\prime}$ & (29) \\
\hline 2295.350 & $20.240 \pm 0.076$ & $r^{\prime}$ & (29) \\
\hline 2194.612 & $16.903 \pm 0.014$ & $i^{\prime}$ & $(29)$ \\
\hline 2194.620 & $16.883 \pm 0.015$ & $i^{\prime}$ & $(29)$ \\
\hline 2197.620 & $17.018 \pm 0.026$ & $i^{\prime}$ & $(29)$ \\
\hline 2197.624 & $17.053 \pm 0.028$ & $i^{\prime}$ & (29) \\
\hline 2198.636 & $17.588 \pm 0.043$ & $i^{\prime}$ & (29) \\
\hline 2198.640 & $17.595 \pm 0.048$ & $i^{\prime}$ & (29) \\
\hline 2199.382 & $17.868 \pm 0.030$ & $i^{\prime}$ & (29) \\
\hline 2199.386 & $17.824 \pm 0.026$ & $i^{\prime}$ & (29) \\
\hline 2200.628 & $17.654 \pm 0.037$ & $i^{\prime}$ & (29) \\
\hline 2200.632 & $17.673 \pm 0.033$ & $i^{\prime}$ & (29) \\
\hline 2202.448 & $17.194 \pm 0.024$ & $i^{\prime}$ & $(29)$ \\
\hline 2202.456 & $17.201 \pm 0.022$ & $i^{\prime}$ & $(29)$ \\
\hline 2230.331 & $18.751 \pm 0.048$ & $i^{\prime}$ & $(29)$ \\
\hline \multicolumn{4}{|c|}{ M31N 2002-08a } \\
\hline 2490.523 & $17.05 \pm 0.1$ & $R$ & (2) \\
\hline 2504.452 & $17.2 \pm 0.1$ & $R$ & (2) \\
\hline 2512.360 & $18.2 \pm 0.2$ & $R$ & (2) \\
\hline 2513.344 & $17.83 \pm 0.09$ & $R$ & (2) \\
\hline 2516.506 & $18.1 \pm 0.1$ & $R$ & $(32)$ \\
\hline 2517.368 & $18.6 \pm 0.2$ & $R$ & $(2)$ \\
\hline 2517.560 & $18.4 \pm 0.15$ & $R$ & $(32)$ \\
\hline
\end{tabular}


Table 3-Continued

\begin{tabular}{lllr}
\hline \hline JD & & & \\
$(2,450,000+)$ & \multicolumn{1}{c}{ Mag } & Filter & Notes $^{\mathrm{a}}$ \\
\hline 2520.452 & $18.4 \pm 0.2$ & $R$ & $(2)$ \\
2521.433 & $18.5 \pm 0.2$ & $R$ & $(2)$ \\
2522.414 & $18.7 \pm 0.2$ & $R$ & $(2)$ \\
2524.358 & $18.5 \pm 0.15$ & $R$ & $(32)$ \\
2525.417 & $19.0 \pm 0.25$ & $R$ & $(2)$ \\
2529.435 & $18.6 \pm 0.2$ & $R$ & $(2)$ \\
2530.438 & $18.7 \pm 0.2$ & $R$ & $(2)$ \\
2530.637 & $18.7 \pm 0.2$ & $R$ & $(32)$ \\
2547.365 & $19.3 \pm 0.3$ & $R$ & $(2)$ \\
2548.505 & $19.0 \pm 0.3$ & $R$ & $(2)$ \\
\hline & & & \\
\hline 3241.563 & $17.6 \pm 0.15$ & $V$ & $(32)$ \\
3220.474 & $>20.5$ & $R$ & $(32)$ \\
3221.460 & $19.2 \pm 0.4$ & $R$ & $(2)$ \\
3222.401 & $18.8 \pm 0.3$ & $R$ & $(2)$ \\
3224.497 & $17.8 \pm 0.15$ & $R$ & $(32)$ \\
3225.405 & $17.4 \pm 0.2$ & $R$ & $(2)$ \\
3225.482 & $17.3 \pm 0.15$ & $R$ & $(32)$ \\
3226.570 & $17.8 \pm 0.15$ & $R$ & $(32)$ \\
3227.505 & $18.3 \pm 0.2$ & $R$ & $(2)$ \\
3228.393 & $18.0 \pm 0.2$ & $R$ & $(2)$ \\
3228.456 & $17.7 \pm 0.15$ & $R$ & $(2)$ \\
3229.557 & $18.3 \pm 0.15$ & $R$ & $(32)$ \\
3233.443 & $17.6 \pm 0.2$ & $R$ & $(2)$ \\
3235.370 & $17.6 \pm 0.15$ & $R$ & $(2)$ \\
3236.410 & $17.7 \pm 0.15$ & $R$ & $(2)$ \\
3236.586 & $17.7 \pm 0.1$ & $R$ & $(32)$ \\
3237.375 & $17.7 \pm 0.15$ & $R$ & $(2)$ \\
3240.429 & $17.6 \pm 0.2$ & $R$ & $(2)$ \\
3241.402 & $17.5 \pm 0.15$ & $R$ & $(2)$ \\
3241.560 & $17.6 \pm 0.15$ & $R$ & $(32)$ \\
3246.342 & $18.3 \pm 0.2$ & $R$ & $(2)$ \\
3246.399 & $18.1 \pm 0.15$ & $R$ & $(2)$ \\
3249.415 & $18.6 \pm 0.2$ & $R$ & $(2)$ \\
3249.464 & $18.5 \pm 0.2$ & $R$ & $(2)$ \\
3251.518 & $18.6 \pm 0.2$ & $R$ & $(32)$ \\
3252.312 & $18.7 \pm 0.25$ & $R$ & $(2)$ \\
3252.368 & $18.7 \pm 0.25$ & $R$ & $(2)$ \\
3253.319 & $19.0 \pm 0.25$ & $R$ & $(2)$ \\
3253.558 & $19.0 \pm 0.2$ & $R$ & $(32)$ \\
3254.362 & $19.0 \pm 0.3$ & $R$ & $(2)$ \\
& $19.0 \pm 0.3$ & $R$ & $(2)$
\end{tabular}


Table 3-Continued

\begin{tabular}{|c|c|c|c|}
\hline $\begin{array}{c}\mathrm{JD} \\
(2,450,000+)\end{array}$ & Mag & Filter & Notes $^{a}$ \\
\hline 3257.429 & $18.3 \pm 0.2$ & $R$ & (2) \\
\hline 3257.453 & $18.4 \pm 0.2$ & $R$ & (2) \\
\hline 3257.571 & $18.5 \pm 0.15$ & $R$ & $(32)$ \\
\hline 3258.363 & $18.4 \pm 0.15$ & $R$ & $(2)$ \\
\hline 3258.393 & $18.3 \pm 0.2$ & $R$ & (2) \\
\hline 3259.384 & $18.6 \pm 0.25$ & $R$ & (2) \\
\hline 3259.413 & $18.4 \pm 0.2$ & $R$ & (2) \\
\hline 3260.385 & $18.6 \pm 0.2$ & $R$ & $(32)$ \\
\hline 3262.453 & $18.6 \pm 0.2$ & $R$ & (2) \\
\hline 3265.332 & $19.4 \pm 0.3$ & $R$ & (2) \\
\hline 3265.352 & $19.5 \pm 0.3$ & $R$ & (2) \\
\hline 3266.335 & $19.4 \pm 0.3$ & $R$ & (2) \\
\hline 3270.330 & $19.6 \pm 0.4$ & $R$ & (2) \\
\hline 3275.290 & $19.6 \pm 0.3$ & $R$ & (2) \\
\hline 3279.356 & $19.6 \pm 0.25$ & $R$ & $(32)$ \\
\hline 3282.281 & $19.4 \pm 0.3$ & $R$ & $(2)$ \\
\hline 3283.619 & $19.5 \pm 0.25$ & $R$ & $(32)$ \\
\hline 3288.272 & $19.2 \pm 0.25$ & $R$ & $(2)$ \\
\hline 3289.828 & $20.40 \pm 0.1$ & $R$ & (33) \\
\hline 3301.408 & $20.2 \pm 0.4$ & $R$ & $(32)$ \\
\hline \multicolumn{4}{|c|}{ M31N 2004-09a } \\
\hline 3241.560 & $>19.8$ & $R$ & $(32)$ \\
\hline 3246.399 & $>19.1$ & $R$ & (2) \\
\hline 3249.415 & $18.3 \pm 0.2$ & $R$ & (2) \\
\hline 3249.438 & $18.0 \pm 0.15$ & $R$ & (2) \\
\hline 3249.452 & $17.8 \pm 0.15$ & $R$ & (2) \\
\hline 3251.518 & $17.5 \pm 0.1$ & $R$ & $(32)$ \\
\hline 3252.312 & $17.7 \pm 0.15$ & $R$ & (2) \\
\hline 3252.368 & $17.6 \pm 0.1$ & $R$ & (2) \\
\hline 3253.319 & $18.3 \pm 0.15$ & $R$ & (2) \\
\hline 3253.341 & $18.2 \pm 0.15$ & $R$ & (2) \\
\hline 3253.441 & $18.0 \pm 0.15$ & $R$ & (2) \\
\hline 3253.558 & $18.1 \pm 0.1$ & $R$ & $(32)$ \\
\hline 3254.362 & $18.1 \pm 0.15$ & $R$ & $(2)$ \\
\hline 3254.400 & $18.2 \pm 0.15$ & $R$ & (2) \\
\hline 3255.430 & $18.0 \pm 0.15$ & $R$ & (2) \\
\hline 3257.429 & $18.2 \pm 0.15$ & $R$ & (2) \\
\hline 3257.453 & $18.1 \pm 0.15$ & $R$ & (2) \\
\hline 3257.571 & $18.2 \pm 0.1$ & $R$ & $(32)$ \\
\hline 3258.363 & $18.3 \pm 0.2$ & $R$ & (2) \\
\hline 3258.393 & $18.4 \pm 0.2$ & $R$ & (2) \\
\hline 3259.384 & $18.6 \pm 0.25$ & $R$ & (2) \\
\hline 3259.413 & $18.4 \pm 0.25$ & $R$ & (2) \\
\hline
\end{tabular}


Table 3-Continued

\begin{tabular}{|c|c|c|c|}
\hline $\begin{array}{c}\text { JD } \\
(2,450,000+)\end{array}$ & Mag & Filter & Notes $^{\mathrm{a}}$ \\
\hline 3260.385 & $18.6 \pm 0.15$ & $R$ & $(32)$ \\
\hline 3262.453 & $18.7 \pm 0.25$ & $R$ & $(2)$ \\
\hline 3262.472 & $18.6 \pm 0.2$ & $R$ & (2) \\
\hline 3265.332 & $18.8 \pm 0.25$ & $R$ & (2) \\
\hline 3265.352 & $18.5 \pm 0.4$ & $R$ & (2) \\
\hline 3266.335 & $19.2 \pm 0.4$ & $R$ & $(2)$ \\
\hline 3270.330 & $18.6 \pm 0.4$ & $R$ & (2) \\
\hline 3270.351 & $19.1 \pm 0.4$ & $R$ & (2) \\
\hline 3275.290 & $19.5 \pm 0.4$ & $R$ & (2) \\
\hline 3279.356 & $19.3 \pm 0.25$ & $R$ & $(32)$ \\
\hline 3279.398 & $19.4 \pm 0.3$ & $R$ & $(2)$ \\
\hline 3282.257 & $19.2 \pm 0.25$ & $R$ & (2) \\
\hline 3283.619 & $19.8 \pm 0.4$ & $R$ & $(32)$ \\
\hline 3288.272 & $19.6 \pm 0.4$ & $R$ & $(2)$ \\
\hline 3288.292 & $20.1 \pm 0.4$ & $R$ & $(2)$ \\
\hline 3289.802 & $20.8 \pm 0.4$ & $R$ & (33) \\
\hline \multicolumn{4}{|c|}{ M31N 2004-11a } \\
\hline 3301.408 & $>21.0$ & $R$ & $(2)$ \\
\hline 3315.347 & $16.6 \pm 0.15$ & $R$ & (2) \\
\hline 3315.390 & $16.5 \pm 0.15$ & $R$ & (2) \\
\hline 3317.352 & $17.0 \pm 0.15$ & $R$ & (2) \\
\hline 3321.404 & $18.1 \pm 0.2$ & $R$ & $(2)$ \\
\hline 3324.305 & $18.2 \pm 0.2$ & $R$ & (2) \\
\hline 3325.218 & $18.4 \pm 0.25$ & $R$ & $(2)$ \\
\hline 3334.218 & $19.0 \pm 0.3$ & $R$ & (2) \\
\hline 3335.273 & $19.2 \pm 0.3$ & $R$ & (2) \\
\hline 3339.296 & $19.4 \pm 0.4$ & $R$ & (2) \\
\hline 3339.318 & $19.6 \pm 0.4$ & $R$ & (2) \\
\hline 3342.192 & $19.3 \pm 0.4$ & $R$ & $(32)$ \\
\hline 3344.192 & $19.9 \pm 0.4$ & $R$ & (2) \\
\hline 3344.214 & $19.3 \pm 0.4$ & $R$ & $(2)$ \\
\hline 3357.568 & $19.8 \pm 0.15$ & $R$ & (37) \\
\hline 3358.260 & $20.5 \pm 0.4$ & $R$ & $(32)$ \\
\hline \multicolumn{4}{|c|}{ M31N 2004-11b } \\
\hline 3381.253 & $19.5 \pm 0.4$ & $V$ & $(32)$ \\
\hline 3301.408 & $>21.0$ & $R$ & (2) \\
\hline 3315.347 & $16.7 \pm 0.1$ & $R$ & $(2)$ \\
\hline 3315.390 & $16.6 \pm 0.1$ & $R$ & (2) \\
\hline 3317.352 & $17.1 \pm 0.15$ & $R$ & $(2)$ \\
\hline 3321.404 & $17.4 \pm 0.15$ & $R$ & (2) \\
\hline
\end{tabular}


Table 3-Continued

\begin{tabular}{|c|c|c|c|}
\hline $\begin{array}{c}\text { JD } \\
(2,450,000+)\end{array}$ & Mag & Filter & Notes $^{\mathrm{a}}$ \\
\hline 3324.305 & $17.3 \pm 0.15$ & $R$ & $(2)$ \\
\hline 3325.218 & $17.2 \pm 0.15$ & $R$ & (2) \\
\hline 3334.218 & $17.5 \pm 0.15$ & $R$ & $(2)$ \\
\hline 3335.273 & $17.6 \pm 0.15$ & $R$ & (2) \\
\hline 3339.296 & $17.9 \pm 0.2$ & $R$ & (2) \\
\hline 3339.318 & $17.9 \pm 0.15$ & $R$ & (2) \\
\hline 3342.172 & $18.2 \pm 0.15$ & $R$ & $(32)$ \\
\hline 3344.192 & $18.1 \pm 0.15$ & $R$ & $(2)$ \\
\hline 3344.214 & $18.2 \pm 0.15$ & $R$ & $(2)$ \\
\hline 3346.410 & $18.3 \pm 0.15$ & $R$ & (2) \\
\hline 3347.344 & $18.5 \pm 0.2$ & $R$ & $(2)$ \\
\hline 3347.370 & $18.8 \pm 0.2$ & $R$ & (2) \\
\hline 3348.359 & $18.7 \pm 0.2$ & $R$ & $(2)$ \\
\hline 3357.568 & $19.1 \pm 0.1$ & $R$ & $(37)$ \\
\hline 3358.260 & $19.2 \pm 0.2$ & $R$ & (32) \\
\hline 3360.236 & $19.3 \pm 0.25$ & $R$ & $(2)$ \\
\hline 3361.324 & $19.1 \pm 0.25$ & $R$ & $(2)$ \\
\hline 3370.230 & $19.5 \pm 0.3$ & $R$ & $(2)$ \\
\hline 3370.267 & $19.3 \pm 0.25$ & $R$ & $(2)$ \\
\hline 3373.321 & $19.4 \pm 0.3$ & $R$ & $(32)$ \\
\hline 3377.266 & $19.5 \pm 0.25$ & $R$ & $(32)$ \\
\hline 3378.391 & $19.7 \pm 0.3$ & $R$ & (2) \\
\hline 3380.426 & $19.7 \pm 0.3$ & $R$ & $(2)$ \\
\hline 3381.249 & $19.5 \pm 0.25$ & $R$ & $(32)$ \\
\hline 3381.276 & $19.4 \pm 0.3$ & $R$ & $(2)$ \\
\hline 3381.417 & $19.4 \pm 0.3$ & $R$ & $(2)$ \\
\hline 3382.219 & $19.3 \pm 0.3$ & $R$ & $(2)$ \\
\hline 3382.246 & $19.3 \pm 0.4$ & $R$ & $(32)$ \\
\hline 3384.212 & $19.6 \pm 0.4$ & $R$ & $(2)$ \\
\hline 3387.231 & $19.8 \pm 0.4$ & $R$ & $(32)$ \\
\hline 3387.400 & $20.0 \pm 0.4$ & $R$ & $(2)$ \\
\hline 3388.224 & $19.5 \pm 0.4$ & $R$ & $(32)$ \\
\hline \multicolumn{4}{|c|}{ M31N 2005-01a } \\
\hline 3381.253 & $15.45 \pm 0.05$ & V & $(32)$ \\
\hline 3382.251 & $15.26 \pm 0.05$ & $V$ & $(32)$ \\
\hline 3384.333 & $15.26 \pm 0.07$ & V & $(32)$ \\
\hline 3386.305 & $15.65 \pm 0.06$ & $V$ & $(32)$ \\
\hline 3387.237 & $15.75 \pm 0.06$ & $V$ & $(32)$ \\
\hline 3388.233 & $15.77 \pm 0.08$ & V & $(32)$ \\
\hline 3373.321 & $>19.8$ & $R$ & $(32)$ \\
\hline 3377.266 & $19.2 \pm 0.2$ & $R$ & $(32)$ \\
\hline 3377.293 & $19.4 \pm 0.3$ & $R$ & $(2)$ \\
\hline
\end{tabular}


Table 3-Continued

\begin{tabular}{cccr}
\hline \hline JD & & & \\
$(2,450,000+)$ & Mag & Filter & Notes $^{\text {a }}$ \\
\hline 3378.391 & $17.9 \pm 0.15$ & $R$ & $(2)$ \\
3380.224 & $15.72 \pm 0.07$ & $R$ & $(2)$ \\
3380.253 & $15.68 \pm 0.06$ & $R$ & $(2)$ \\
3380.437 & $15.33 \pm 0.07$ & $R$ & $(2)$ \\
3381.249 & $15.48 \pm 0.04$ & $R$ & $(32)$ \\
3381.266 & $15.32 \pm 0.06$ & $R$ & $(2)$ \\
3381.286 & $15.27 \pm 0.05$ & $R$ & $(2)$ \\
3381.306 & $15.30 \pm 0.04$ & $R$ & $(2)$ \\
3381.408 & $15.34 \pm 0.07$ & $R$ & $(2)$ \\
3381.426 & $15.31 \pm 0.06$ & $R$ & $(2)$ \\
3382.219 & $15.16 \pm 0.05$ & $R$ & $(2)$ \\
3382.246 & $15.21 \pm 0.05$ & $R$ & $(32)$ \\
3382.257 & $15.21 \pm 0.06$ & $R$ & $(2)$ \\
3384.212 & $15.05 \pm 0.05$ & $R$ & $(2)$ \\
3384.252 & $15.10 \pm 0.06$ & $R$ & $(2)$ \\
3384.326 & $15.12 \pm 0.05$ & $R$ & $(32)$ \\
3384.405 & $15.04 \pm 0.07$ & $R$ & $(2)$ \\
3386.202 & $15.27 \pm 0.06$ & $R$ & $(2)$ \\
3386.228 & $15.26 \pm 0.05$ & $R$ & $(2)$ \\
3386.300 & $15.27 \pm 0.04$ & $R$ & $(32)$ \\
3386.424 & $15.31 \pm 0.05$ & $R$ & $(2)$ \\
3387.212 & $15.36 \pm 0.04$ & $R$ & $(2)$ \\
3387.231 & $15.39 \pm 0.05$ & $R$ & $(32)$ \\
3387.249 & $15.34 \pm 0.06$ & $R$ & $(2)$ \\
3387.400 & $15.34 \pm 0.05$ & $R$ & $(2)$ \\
3388.224 & $15.42 \pm 0.05$ & $R$ & $(32)$ \\
3390.287 & $15.52 \pm 0.07$ & $R$ & $(2)$ \\
3390.327 & $15.50 \pm 0.06$ & $R$ & $(2)$ \\
3394.234 & $15.80 \pm 0.07$ & $R$ & $(2)$ \\
3398.274 & $16.61 \pm 0.08$ & $R$ & $(2)$ \\
3401.212 & $17.17 \pm 0.09$ & $R$ & $(2)$ \\
3405.273 & $19.4 \pm 0.4$ & $R$ & $(32)$ \\
3405.633 & $19.7 \pm 0.15$ & $R$ & $(20)$ \\
3406.326 & $20.1 \pm 0.25$ & $R$ & $(32)$ \\
3407.231 & $>19.9$ & $R$ & $(2)$ \\
3407.283 & $>19.8$ & $R$ & $(2)$ \\
3509.952 & $21.7 \pm 0.25$ & $R$ & $(33)$ \\
3411.257 & $>20.3$ & $R$ & $(32)$ \\
3532.699 & $21.13 \pm 0.09$ & $R$ & $(38)$ \\
3534.701 & $21.38 \pm 0.1$ & $R$ & $(38)$ \\
3535.681 & $21.45 \pm 0.1$ & $R$ & $(38)$ \\
3538.697 & $21.43 \pm 0.1$ & $R$ & $(38)$ \\
3541.718 & $21.2 \pm 0.15$ & $R$ & $(38)$ \\
3651.821 & $22.0 \pm 0.4$ & $R$ & $(35)$ \\
3702.638 & $>21.3$ & $R$ & $(33)$
\end{tabular}


Table 3-Continued

\begin{tabular}{|c|c|c|c|}
\hline $\begin{array}{c}\text { JD } \\
(2,450,000+)\end{array}$ & Mag & Filter & Notes $^{\mathrm{a}}$ \\
\hline 3710.730 & $21.9 \pm 0.25$ & $R$ & $(36)$ \\
\hline 3996.839 & $>23.0$ & $R$ & $(37)$ \\
\hline 3384.315 & $14.95 \pm 0.07$ & $I$ & $(2)$ \\
\hline \multicolumn{4}{|c|}{ M31N 2005-07a } \\
\hline 3564.493 & $>19.5$ & $R$ & $(32)$ \\
\hline 3575.429 & $>19.0$ & $R$ & $(2)$ \\
\hline 3579.409 & $18.4 \pm 0.25$ & $R$ & (2) \\
\hline 3581.419 & $17.4 \pm 0.15$ & $R$ & (2) \\
\hline 3584.404 & $19.1 \pm 0.3$ & $R$ & $(2)$ \\
\hline 3587.509 & $19.3 \pm 0.25$ & $R$ & $(32)$ \\
\hline 3588.415 & $18.8 \pm 0.25$ & $R$ & $(2)$ \\
\hline 3594.390 & $19.7 \pm 0.35$ & $R$ & $(32)$ \\
\hline 3651.862 & $19.53 \pm 0.05$ & $R$ & $(32)$ \\
\hline 3702.638 & $20.8 \pm 0.2$ & $R$ & $(33)$ \\
\hline 3710.730 & $21.03 \pm 0.12$ & $R$ & $(36)$ \\
\hline 3760.586 & $21.8 \pm 0.3$ & $R$ & $(36)$ \\
\hline 3771.346 & $21.7 \pm 0.3$ & $R$ & (21) \\
\hline \multicolumn{4}{|c|}{ M31N 2006-06a } \\
\hline 3771.264 & $>19.2$ & $R$ & $(2)$ \\
\hline 3771.346 & $>22$ & $R$ & $(21)$ \\
\hline 3869.565 & $>19.8$ & $R$ & $(1)$ \\
\hline 3892.518 & $17.6 \pm 0.1$ & $R$ & (3) \\
\hline 3899.502 & $18.0 \pm 0.15$ & $R$ & (1) \\
\hline 3899.540 & $17.9 \pm 0.15$ & $R$ & (1) \\
\hline 3900.502 & $18.1 \pm 0.15$ & $R$ & (1) \\
\hline 3911.522 & $18.5 \pm 0.25$ & $R$ & (2) \\
\hline 3921.464 & $19.2 \pm 0.25$ & $R$ & (2) \\
\hline \multicolumn{4}{|c|}{ M31N 2006-09c } \\
\hline 4256.677 & $>21.2$ & $B$ & $(30)$ \\
\hline 4260.700 & $>21.7$ & $B$ & (30) \\
\hline 4260.705 & $>21.0$ & $V$ & $(30)$ \\
\hline 3892.967 & $>21.1$ & $R$ & (14) \\
\hline 3991.566 & $>20.0$ & $R$ & $(4)$ \\
\hline 3993.376 & $>19.1$ & $R$ & (2) \\
\hline 3996.404 & $18.1 \pm 0.2$ & $R$ & (3) \\
\hline 3999.609 & $17.15 \pm 0.1$ & $R$ & (3) \\
\hline
\end{tabular}


Table 3-Continued

\begin{tabular}{|c|c|c|c|}
\hline $\begin{array}{c}\text { JD } \\
(2,450,000+)\end{array}$ & Mag & Filter & Notes $^{\mathrm{a}}$ \\
\hline 4000.317 & $17.0 \pm 0.1$ & $R$ & (2) \\
\hline 4000.590 & $17.35 \pm 0.1$ & $R$ & (4) \\
\hline 4001.360 & $17.3 \pm 0.1$ & $R$ & (2) \\
\hline 4002.302 & $17.4 \pm 0.1$ & $R$ & (2) \\
\hline 4002.329 & $17.5 \pm 0.1$ & $R$ & (2) \\
\hline 4005.312 & $17.8 \pm 0.1$ & $R$ & (2) \\
\hline 4007.312 & $17.9 \pm 0.1$ & $R$ & (2) \\
\hline 4014.295 & $19.0 \pm 0.2$ & $R$ & (2) \\
\hline 4017.258 & $19.1 \pm 0.2$ & $R$ & (2) \\
\hline 4019.319 & $18.8 \pm 0.2$ & $R$ & (2) \\
\hline 4024.383 & $19.3 \pm 0.2$ & $R$ & (2) \\
\hline 4026.330 & $19.3 \pm 0.2$ & $R$ & (2) \\
\hline 4026.364 & $19.3 \pm 0.2$ & $R$ & (2) \\
\hline 4034.312 & $19.9 \pm 0.3$ & $R$ & (2) \\
\hline 4260.690 & $>21.4$ & $r^{\prime}$ & $(30)$ \\
\hline \multirow[t]{2}{*}{4260.695} & $21.800 \pm 0.500$ & $i^{\prime}$ & $(30)$ \\
\hline & \multicolumn{3}{|l|}{ M31N 2006-10a } \\
\hline 4044.337 & $18.072 \pm 0.035$ & $B$ & (30) \\
\hline 4050.589 & $19.064 \pm 0.033$ & $B$ & $(30)$ \\
\hline 4056.585 & $18.879 \pm 0.032$ & $B$ & (30) \\
\hline 4062.606 & $18.579 \pm 0.030$ & $B$ & $(30)$ \\
\hline 4069.484 & $19.240 \pm 0.044$ & $B$ & (30) \\
\hline 4071.549 & $19.055 \pm 0.091$ & $B$ & (30) \\
\hline 4074.575 & $19.201 \pm 0.106$ & $B$ & $(30)$ \\
\hline 4077.542 & $19.255 \pm 0.132$ & $B$ & (30) \\
\hline 4084.460 & $19.239 \pm 0.034$ & $B$ & $(30)$ \\
\hline 4092.454 & $19.933 \pm 0.054$ & $B$ & (30) \\
\hline 4099.407 & $20.033 \pm 0.108$ & $B$ & $(30)$ \\
\hline 4101.393 & $19.781 \pm 0.071$ & $B$ & (30) \\
\hline 4114.372 & $>21.896$ & $B$ & (30) \\
\hline 4120.432 & $>20.893$ & $B$ & (30) \\
\hline 4254.685 & $>17.6$ & $B$ & (30) \\
\hline 4044.334 & $17.904 \pm 0.026$ & $V$ & (30) \\
\hline 4050.586 & $19.096 \pm 0.024$ & $V$ & $(30)$ \\
\hline 4056.582 & $18.797 \pm 0.025$ & $V$ & (30) \\
\hline 4062.604 & $18.475 \pm 0.021$ & $V$ & $(30)$ \\
\hline 4069.481 & $19.253 \pm 0.039$ & $V$ & (30) \\
\hline 4071.546 & $19.022 \pm 0.118$ & $V$ & (30) \\
\hline 4074.572 & $19.387 \pm 0.099$ & $V$ & $(30)$ \\
\hline 4077.539 & $19.299 \pm 0.045$ & $V$ & (30) \\
\hline
\end{tabular}


Table 3-Continued

\begin{tabular}{|c|c|c|c|}
\hline $\begin{array}{c}\text { JD } \\
(2,450,000+)\end{array}$ & Mag & Filter & Notes $^{\mathrm{a}}$ \\
\hline 4084.457 & $19.161 \pm 0.027$ & $V$ & $(30)$ \\
\hline 4092.451 & $19.666 \pm 0.055$ & $V$ & (30) \\
\hline 4099.404 & $19.638 \pm 0.096$ & $V$ & $(30)$ \\
\hline 4101.391 & $19.734 \pm 0.058$ & $V$ & (30) \\
\hline 4114.370 & $>21.545$ & $V$ & (30) \\
\hline 4120.429 & $>21.221$ & $V$ & (30) \\
\hline 4254.692 & $>18.4$ & $V$ & $(30)$ \\
\hline 3771.346 & $>22$ & $R$ & $(21)$ \\
\hline 4019.319 & $>19.7$ & $R$ & (2) \\
\hline 4024.383 & $>19.0$ & $R$ & (2) \\
\hline 4026.330 & $>20.1$ & $R$ & (2) \\
\hline 4031.251 & $19.2 \pm 0.25$ & $R$ & (2) \\
\hline 4034.312 & $18.7 \pm 0.15$ & $R$ & (2) \\
\hline 4034.470 & $18.6 \pm 0.15$ & $R$ & (3) \\
\hline 4035.360 & $18.4 \pm 0.3$ & $R$ & (2) \\
\hline 4043.331 & $17.9 \pm 0.1$ & $R$ & (2) \\
\hline 4047.288 & $19.0 \pm 0.3$ & $R$ & (2) \\
\hline 4048.324 & $19.3 \pm 0.2$ & $R$ & (2) \\
\hline 4055.296 & $18.2 \pm 0.15$ & $R$ & (2) \\
\hline 4055.262 & $18.2 \pm 0.1$ & $R$ & (23) \\
\hline 4070.308 & $19.2 \pm 0.35$ & $R$ & (6) \\
\hline 4071.385 & $18.9 \pm 0.2$ & $R$ & (3) \\
\hline 4078.308 & $18.4 \pm 0.3$ & $R$ & (2) \\
\hline 4078.343 & $18.6 \pm 0.2$ & $R$ & (2) \\
\hline 4080.306 & $18.8 \pm 0.25$ & $R$ & (2) \\
\hline 4084.212 & $18.9 \pm 0.2$ & $R$ & (2) \\
\hline 4093.174 & $19.5: \pm 0.4$ & $R$ & (2) \\
\hline 4096.325 & $19.7 \pm 0.25$ & $R$ & (2) \\
\hline 4097.222 & $19.5 \pm 0.25$ & $R$ & (2) \\
\hline 4115.194 & $>20.0$ & $R$ & (2) \\
\hline 4121.381 & $>19.8$ & $R$ & (2) \\
\hline 4122.331 & $>19.5$ & $R$ & (2) \\
\hline 4122.377 & $>19.4$ & $R$ & (2) \\
\hline 4254.671 & $>19.0$ & $r^{\prime}$ & $(30)$ \\
\hline \multicolumn{4}{|c|}{ M31N 2006-10b } \\
\hline 4044.388 & $18.952 \pm 0.057$ & $B$ & $(30)$ \\
\hline 4049.501 & $19.553 \pm 0.042$ & $B$ & $(30)$ \\
\hline 4057.534 & $20.385 \pm 0.057$ & $B$ & (30) \\
\hline 4063.463 & $20.892 \pm 0.066$ & $B$ & (30) \\
\hline 4069.445 & $20.989 \pm 0.129$ & $B$ & $(30)$ \\
\hline 4072.440 & $>20.144$ & $B$ & (30) \\
\hline
\end{tabular}


Table 3-Continued

\begin{tabular}{|c|c|c|c|}
\hline $\begin{array}{c}\text { JD } \\
(2,450,000+)\end{array}$ & Mag & Filter & Notes $^{\mathrm{a}}$ \\
\hline 4075.473 & $>20.354$ & $B$ & $(30)$ \\
\hline 4084.411 & $21.410 \pm 0.103$ & $B$ & (30) \\
\hline 4099.438 & $>20.647$ & $B$ & (30) \\
\hline 4106.415 & $>19.739$ & $B$ & (30) \\
\hline 4114.383 & $>22.913$ & $B$ & (30) \\
\hline 4120.458 & $>21.830$ & $B$ & (30) \\
\hline 4248.704 & $>22.422$ & $B$ & $(30)$ \\
\hline 4049.498 & $20.021 \pm 0.045$ & $V$ & (30) \\
\hline 4054.317 & $>17.332$ & $V$ & (30) \\
\hline 4057.531 & $20.881 \pm 0.072$ & $V$ & $(30)$ \\
\hline 4063.460 & $21.271 \pm 0.077$ & $V$ & $(30)$ \\
\hline 4069.442 & $>21.692$ & $V$ & (30) \\
\hline 4072.437 & $>20.121$ & $V$ & $(30)$ \\
\hline 4075.470 & $>20.641$ & $V$ & (30) \\
\hline 4084.408 & $>22.297$ & $V$ & (30) \\
\hline 4099.436 & $>20.506$ & $V$ & (30) \\
\hline 4102.385 & $>20.134$ & $V$ & (30) \\
\hline 4106.412 & $>17.613$ & $V$ & $(30)$ \\
\hline 4108.486 & $>18.404$ & $V$ & $(30)$ \\
\hline 4114.380 & $>21.955$ & $V$ & (30) \\
\hline 4120.455 & $>22.758$ & $V$ & $(30)$ \\
\hline 4248.709 & $>22.304$ & $V$ & (30) \\
\hline 4248.693 & $>22.9$ & $r^{\prime}$ & $(30)$ \\
\hline \multirow[t]{2}{*}{4248.699} & $>21.5$ & $i^{\prime}$ & $(30)$ \\
\hline & \multicolumn{3}{|l|}{ M31N 2006-11a } \\
\hline 4141.368 & $20.120 \pm 0.050$ & $B$ & $(30)$ \\
\hline 4141.372 & $20.540 \pm 0.060$ & $V$ & $(30)$ \\
\hline 3771.346 & $>22$ & $R$ & $(21)$ \\
\hline 4048.324 & $>19.8$ & $R$ & $(2)$ \\
\hline 4055.296 & $>19.8$ & $R$ & (2) \\
\hline 4070.263 & $16.9 \pm 0.1$ & $R$ & (6) \\
\hline 4070.308 & $16.6 \pm 0.15$ & $R$ & (6) \\
\hline 4078.308 & $16.1 \pm 0.1$ & $R$ & (2) \\
\hline 4078.343 & $16.0 \pm 0.1$ & $R$ & (2) \\
\hline 4080.306 & $16.3 \pm 0.1$ & $R$ & (2) \\
\hline 4084.212 & $16.9 \pm 0.1$ & $R$ & (2) \\
\hline 4093.174 & $17.7 \pm 0.15$ & $R$ & (2) \\
\hline 4096.325 & $17.8 \pm 0.15$ & $R$ & (2) \\
\hline
\end{tabular}


Table 3-Continued

\begin{tabular}{|c|c|c|c|}
\hline $\begin{array}{c}\mathrm{JD} \\
(2,450,000+)\end{array}$ & Mag & Filter & Notes $^{\mathrm{a}}$ \\
\hline 4097.222 & $17.8 \pm 0.15$ & $R$ & $(2)$ \\
\hline 4115.194 & $18.8 \pm 0.2$ & $R$ & (2) \\
\hline 4121.381 & $18.9 \pm 0.2$ & $R$ & (2) \\
\hline 4122.331 & $19.2 \pm 0.25$ & $R$ & (2) \\
\hline 4122.377 & $19.1 \pm 0.3$ & $R$ & (2) \\
\hline 4126.289 & $19.3 \pm 0.3$ & $R$ & (2) \\
\hline 4126.339 & $19.1 \pm 0.3$ & $R$ & (2) \\
\hline 4128.275 & $19.2 \pm 0.25$ & $R$ & (2) \\
\hline 4135.298 & $19.4 \pm 0.3$ & $R$ & (2) \\
\hline 4135.334 & $19.3 \pm 0.25$ & $R$ & (2) \\
\hline 4141.362 & $19.2 \pm 0.3$ & $R$ & (2) \\
\hline 4146.295 & $19.4 \pm 0.25$ & $R$ & (2) \\
\hline 4149.273 & $19.4 \pm 0.25$ & $R$ & (2) \\
\hline 4166.247 & $19.7 \pm 0.2$ & $R$ & (1) \\
\hline 4167.366 & $20.0 \pm 0.35$ & $R$ & (1) \\
\hline 4170.264 & $20.0 \pm 0.3$ & $R$ & (1) \\
\hline 4173.269 & $19.8 \pm 0.25$ & $R$ & $(25)$ \\
\hline 4174.268 & $>19.7$ & $R$ & $(2)$ \\
\hline 4175.267 & $>19.7$ & $R$ & (4) \\
\hline 4240.561 & $>20.0$ & $R$ & (1) \\
\hline 4141.356 & $19.220 \pm 0.030$ & $r^{\prime}$ & $(30)$ \\
\hline \multirow[t]{2}{*}{4141.362} & $20.220 \pm 0.080$ & $i^{\prime}$ & $(30)$ \\
\hline & \multicolumn{3}{|l|}{ M31N 2006-12a } \\
\hline 3771.346 & $>22$ & $R$ & $(21)$ \\
\hline 4078.343 & $>19.8$ & $R$ & $(2)$ \\
\hline 4080.306 & $>19.9$ & $R$ & (2) \\
\hline 4084.212 & $>20.2$ & $R$ & (2) \\
\hline 4093.174 & $17.3 \pm 0.15$ & $R$ & (2) \\
\hline 4096.325 & $17.4 \pm 0.15$ & $R$ & (2) \\
\hline 4096.357 & $17.5 \pm 0.15$ & $R$ & (2) \\
\hline 4097.222 & $17.8 \pm 0.15$ & $R$ & (2) \\
\hline 4097.241 & $17.8 \pm 0.15$ & $R$ & (2) \\
\hline 4115.194 & $18.8 \pm 0.2$ & $R$ & (2) \\
\hline 4115.226 & $18.6 \pm 0.25$ & $R$ & (2) \\
\hline 4121.381 & $18.9 \pm 0.25$ & $R$ & (2) \\
\hline 4122.331 & $19.1 \pm 0.25$ & $R$ & (2) \\
\hline 4122.377 & $19.1 \pm 0.3$ & $R$ & (2) \\
\hline 4126.289 & $>19.2$ & $R$ & (2) \\
\hline 4126.339 & $>19.2$ & $R$ & (2) \\
\hline 4128.275 & $19.3 \pm 0.3$ & $R$ & (2) \\
\hline
\end{tabular}


Table 3-Continued

\begin{tabular}{|c|c|c|c|}
\hline $\begin{array}{c}\text { JD } \\
(2,450,000+)\end{array}$ & Mag & Filter & Notes $^{\mathrm{a}}$ \\
\hline \multicolumn{4}{|c|}{ M31N 2007-02b } \\
\hline 3771.346 & $>22$ & $R$ & $(21)$ \\
\hline 4128.275 & $>19.9$ & $R$ & $(2)$ \\
\hline 4135.298 & $16.66 \pm 0.1$ & $R$ & (2) \\
\hline 4141.362 & $17.3 \pm 0.15$ & $R$ & (2) \\
\hline 4146.295 & $17.5 \pm 0.15$ & $R$ & (2) \\
\hline 4149.273 & $17.7 \pm 0.1$ & $R$ & (2) \\
\hline 4162.295 & $18.5 \pm 0.2$ & $R$ & (1) \\
\hline 4164.263 & $18.4 \pm 0.2$ & $R$ & (1) \\
\hline 4166.274 & $18.4 \pm 0.15$ & $R$ & (1) \\
\hline 4170.274 & $19.1 \pm 0.2$ & $R$ & (1) \\
\hline 4174.268 & $18.9 \pm 0.25$ & $R$ & (2) \\
\hline 4175.267 & $19.2 \pm 0.3$ & $R$ & (4) \\
\hline 4238.560 & $>19.7$ & $R$ & (1) \\
\hline \multicolumn{4}{|c|}{ M31N 2007-07c } \\
\hline 3771.346 & $>22$ & $R$ & $(21)$ \\
\hline 4288.506 & $>20.0$ & $R$ & (1) \\
\hline 4327.469 & $19.2 \pm 0.25$ & $R$ & (1) \\
\hline 4330.344 & $19.2 \pm 0.25$ & $R$ & (1) \\
\hline \multicolumn{4}{|c|}{ M31N 2007-07e } \\
\hline 3771.346 & $>22$ & $R$ & $(21)$ \\
\hline 4288.506 & $>19.5$ & $R$ & (1) \\
\hline 4327.469 & $17.9 \pm 0.25$ & $R$ & (1) \\
\hline 4330.344 & $18.1 \pm 0.25$ & $R$ & (1) \\
\hline 4343.487 & $18.4 \pm 0.25$ & $R$ & (4) \\
\hline 4353.409 & $18.4 \pm 0.3$ & $R$ & (1) \\
\hline 4356.430 & $19.3 \pm 0.3$ & $R$ & (1) \\
\hline 4357.473 & $19.2 \pm 0.3$ & $R$ & (1) \\
\hline 4358.284 & $19.5 \pm 0.3$ & $R$ & (1) \\
\hline 4365.319 & $19.6 \pm 0.3$ & $R$ & (3) \\
\hline \multicolumn{4}{|c|}{ M31N 2007-08d } \\
\hline 3771.346 & $>22$ & $R$ & $(21)$ \\
\hline 4380.401 & $18.6 \pm 0.2$ & $R$ & $(24)$ \\
\hline 4380.424 & $18.5 \pm 0.15$ & $R$ & (24) \\
\hline 4382.235 & $19.0 \pm 0.25$ & $R$ & (24) \\
\hline 4387.219 & $>19.5$ & $R$ & (24) \\
\hline 4387.231 & $19.5 \pm 0.3$ & $R$ & (24) \\
\hline 4387.561 & $19.6 \pm 0.2$ & $R$ & (1) \\
\hline
\end{tabular}


Table 3-Continued

\begin{tabular}{cccr}
\hline \hline JD & & & \\
$(2,450,000+)$ & Mag & Filter & Notes $^{\mathrm{a}}$ \\
\hline 4388.227 & $19.8 \pm 0.25$ & $R$ & $(24)$ \\
4388.626 & $19.6 \pm 0.35$ & $R$ & $(1)$ \\
4389.233 & $19.8 \pm 0.3$ & $R$ & $(24)$ \\
4389.646 & $20.0 \pm 0.3$ & $R$ & $(1)$ \\
\hline & M31N $2007-10 \mathrm{a}$ & & \\
\hline 4383.529 & $17.856 \pm 0.031$ & $B$ & $(30)$ \\
4384.398 & $18.019 \pm 0.031$ & $B$ & $(30)$ \\
4387.398 & $18.932 \pm 0.034$ & $B$ & $(30)$ \\
4389.384 & $19.276 \pm 0.035$ & $B$ & $(30)$ \\
4390.355 & $19.501 \pm 0.038$ & $B$ & $(30)$ \\
4392.486 & $20.386 \pm 0.048$ & $B$ & $(30)$ \\
4393.714 & $21.077 \pm 0.070$ & $B$ & $(30)$ \\
4394.448 & $21.434 \pm 0.093$ & $B$ & $(30)$ \\
4395.553 & $>20.359$ & $B$ & $(30)$ \\
4396.352 & $>20.224$ & $B$ & $(30)$ \\
4397.375 & $>22.588$ & $B$ & $(30)$ \\
4398.367 & $>22.575$ & $B$ & $(30)$ \\
4399.380 & $>21.985$ & $B$ & $(30)$ \\
4400.693 & $>20.718$ & $B$ & $(30)$ \\
4402.606 & $>22.359$ & $B$ & $(30)$ \\
4405.649 & $>22.927$ & $B$ & $(30)$ \\
4406.679 & $>21.795$ & $B$ & $(30)$ \\
4407.573 & $>22.505$ & $B$ & $(30)$ \\
4410.416 & $>22.726$ & $B$ & $(30)$ \\
4383.860 & $18.091 \pm 0.041$ & $B$ & $(31)$ \\
4384.846 & $18.253 \pm 0.042$ & $B$ & $(31)$ \\
4385.970 & $19.059 \pm 0.060$ & $B$ & $(31)$ \\
4386.811 & $18.922 \pm 0.052$ & $B$ & $(31)$ \\
4387.869 & $19.009 \pm 0.057$ & $B$ & $(31)$ \\
4388.786 & $19.180 \pm 0.062$ & $B$ & $(31)$ \\
4389.991 & $17.651 \pm 0.037$ & $B$ & $(31)$ \\
4390.773 & $19.720 \pm 0.073$ & $B$ & $(31)$ \\
4391.745 & $20.264 \pm 0.094$ & $B$ & $(31)$ \\
4392.739 & $20.398 \pm 0.129$ & $B$ & $(31)$ \\
4393.971 & $>21.016$ & $B$ & $(3)$ \\
4394.793 & $>21.294$ & $B$ & $(31)$ \\
4383.532 & $17.859 \pm 0.036$ & $V$ & $(30)$ \\
4384.401 & $18.021 \pm 0.037$ & $V$ & $(30)$ \\
4387.403 & $18.802 \pm 0.040$ & $V$ & $(30)$ \\
4389.386 & $19.188 \pm 0.040$ & $V$ & $(30)$ \\
4390.358 & $19.372 \pm 0.041$ & $V$ & $(30)$ \\
& & &
\end{tabular}


Table 3-Continued

\begin{tabular}{|c|c|c|c|}
\hline $\begin{array}{c}\text { JD } \\
(2,450,000+)\end{array}$ & Mag & Filter & Notes $^{\mathrm{a}}$ \\
\hline 4392.489 & $20.141 \pm 0.048$ & $V$ & (30) \\
\hline 4393.717 & $20.709 \pm 0.077$ & $V$ & $(30)$ \\
\hline 4394.451 & $20.871 \pm 0.072$ & $V$ & (30) \\
\hline 4395.555 & $>20.255$ & $V$ & (30) \\
\hline 4396.355 & $>20.328$ & $V$ & (30) \\
\hline 4397.378 & $>21.711$ & $V$ & (30) \\
\hline 4398.370 & $>21.835$ & $V$ & (30) \\
\hline 4399.383 & $>22.252$ & $V$ & (30) \\
\hline 4400.696 & $>21.472$ & $V$ & (30) \\
\hline 4402.609 & $>21.735$ & $V$ & (30) \\
\hline 4405.652 & $>22.490$ & $V$ & (30) \\
\hline 4406.682 & $>22.278$ & $V$ & (30) \\
\hline 4407.576 & $>21.828$ & $V$ & (30) \\
\hline 4410.419 & $>21.934$ & $V$ & (30) \\
\hline 4383.862 & $17.933 \pm 0.041$ & $V$ & $(31)$ \\
\hline 4384.849 & $18.141 \pm 0.042$ & $V$ & (31) \\
\hline 4385.973 & $18.808 \pm 0.048$ & $V$ & $(31)$ \\
\hline 4386.813 & $18.796 \pm 0.047$ & $V$ & (31) \\
\hline 4387.872 & $18.962 \pm 0.049$ & $V$ & (31) \\
\hline 4388.789 & $19.047 \pm 0.053$ & $V$ & (31) \\
\hline 4389.994 & $18.163 \pm 0.042$ & $V$ & (31) \\
\hline 4390.777 & $19.512 \pm 0.058$ & $V$ & (31) \\
\hline 4391.748 & $19.834 \pm 0.063$ & $V$ & (31) \\
\hline 4392.742 & $20.394 \pm 0.107$ & $V$ & $(31)$ \\
\hline 4393.975 & $>21.105$ & $V$ & (31) \\
\hline 4394.796 & $>20.904$ & $V$ & (31) \\
\hline 3771.346 & $>22$ & $R$ & $(21)$ \\
\hline 4388.644 & $18.6 \pm 0.2$ & $R$ & (1) \\
\hline 4383.535 & $17.994 \pm 0.027$ & $i^{\prime}$ & (30) \\
\hline 4384.404 & $18.155 \pm 0.027$ & $i^{\prime}$ & (30) \\
\hline 4389.389 & $19.227 \pm 0.032$ & $i^{\prime}$ & (30) \\
\hline 4390.361 & $19.309 \pm 0.032$ & $i^{\prime}$ & (30) \\
\hline 4392.492 & $19.992 \pm 0.044$ & $i^{\prime}$ & (30) \\
\hline 4393.720 & $20.565 \pm 0.071$ & $i^{\prime}$ & (30) \\
\hline 4394.453 & $20.746 \pm 0.066$ & $i^{\prime}$ & (30) \\
\hline 4395.558 & $>21.657$ & $i^{\prime}$ & (30) \\
\hline 4396.358 & $>21.196$ & $i^{\prime}$ & (30) \\
\hline 4397.381 & $>22.289$ & $i^{\prime}$ & (30) \\
\hline 4398.373 & $>21.485$ & $i^{\prime}$ & (30) \\
\hline 4399.386 & $>21.672$ & $i^{\prime}$ & (30) \\
\hline 4400.698 & $>21.071$ & $i^{\prime}$ & (30) \\
\hline 4402.612 & $>21.385$ & $i^{\prime}$ & (30) \\
\hline
\end{tabular}


Table 3-Continued

\begin{tabular}{|c|c|c|c|}
\hline $\begin{array}{c}\text { JD } \\
(2,450,000+)\end{array}$ & Mag & Filter & Notes $^{\mathrm{a}}$ \\
\hline 4405.655 & $>21.920$ & $i^{\prime}$ & $(30)$ \\
\hline 4406.685 & $>21.647$ & $i^{\prime}$ & (30) \\
\hline 4407.578 & $>21.377$ & $i^{\prime}$ & (30) \\
\hline 4410.422 & $>21.955$ & $i^{\prime}$ & (30) \\
\hline 4383.857 & $18.089 \pm 0.032$ & $i^{\prime}$ & $(31)$ \\
\hline 4384.844 & $18.211 \pm 0.033$ & $i^{\prime}$ & $(31)$ \\
\hline 4385.967 & $18.565 \pm 0.035$ & $i^{\prime}$ & $(31)$ \\
\hline 4386.808 & $18.863 \pm 0.037$ & $i^{\prime}$ & $(31)$ \\
\hline 4387.835 & $19.048 \pm 0.051$ & $i^{\prime}$ & (31) \\
\hline 4387.866 & $19.050 \pm 0.042$ & $i^{\prime}$ & $(31)$ \\
\hline 4388.783 & $19.233 \pm 0.043$ & $i^{\prime}$ & $(31)$ \\
\hline 4389.988 & $18.186 \pm 0.031$ & $i^{\prime}$ & $(31)$ \\
\hline 4390.771 & $19.438 \pm 0.046$ & $i^{\prime}$ & $(31)$ \\
\hline 4391.743 & $19.709 \pm 0.049$ & $i^{\prime}$ & (31) \\
\hline 4392.736 & $20.186 \pm 0.082$ & $i^{\prime}$ & $(31)$ \\
\hline 4393.968 & $20.821 \pm 0.123$ & $i^{\prime}$ & $(31)$ \\
\hline 4394.790 & $>20.323$ & $i^{\prime}$ & (31) \\
\hline 4395.810 & $>20.778$ & $i^{\prime}$ & (31) \\
\hline \multicolumn{4}{|c|}{ M31N 2007-10b } \\
\hline 4389.507 & $19.603 \pm 0.049$ & $B$ & (30) \\
\hline 4392.397 & $>22.280$ & $B$ & (30) \\
\hline 4393.519 & $>21.828$ & $B$ & $(30)$ \\
\hline 4394.436 & $>22.276$ & $B$ & $(30)$ \\
\hline 4395.483 & $>21.807$ & $B$ & (30) \\
\hline 4389.504 & $19.927 \pm 0.038$ & $V$ & $(30)$ \\
\hline 4392.394 & $>21.704$ & $V$ & (30) \\
\hline 4393.516 & $>21.935$ & $V$ & (30) \\
\hline 4394.433 & $>22.293$ & $V$ & (30) \\
\hline 4395.480 & $>22.240$ & $V$ & (30) \\
\hline 3771.346 & $>22$ & $R$ & $(21)$ \\
\hline 4382.235 & $>19.4$ & $R$ & $(1)$ \\
\hline 4387.219 & $18.5 \pm 0.15$ & $R$ & $(24)$ \\
\hline 4387.561 & $18.2 \pm 0.1$ & $R$ & $(1)$ \\
\hline 4388.227 & $19.1 \pm 0.2$ & $R$ & $(24)$ \\
\hline 4388.626 & $19.1 \pm 0.2$ & $R$ & (1) \\
\hline 4389.233 & $19.3 \pm 0.2$ & $R$ & $(24)$ \\
\hline 4389.646 & $19.6 \pm 0.25$ & $R$ & $(1)$ \\
\hline 4405.318 & $>19.6$ & $R$ & $(28)$ \\
\hline 4409.388 & $>19.9$ & $R$ & (1) \\
\hline 4410.204 & $>19.7 \pm 0.25$ & $R$ & (27) \\
\hline
\end{tabular}


Table 3-Continued

\begin{tabular}{|c|c|c|c|}
\hline $\begin{array}{c}\text { JD } \\
(2,450,000+)\end{array}$ & Mag & Filter & Notes $^{\mathrm{a}}$ \\
\hline 4411.517 & $>19.7 \pm 0.25$ & $R$ & $(27)$ \\
\hline 4389.501 & $20.148 \pm 0.062$ & $i^{\prime}$ & $(30)$ \\
\hline 4390.406 & $20.649 \pm 0.096$ & $i^{\prime}$ & $(30)$ \\
\hline 4392.391 & $>20.840$ & $i^{\prime}$ & (30) \\
\hline 4393.513 & $>20.811$ & $i^{\prime}$ & (30) \\
\hline 4394.430 & $>21.046$ & $i^{\prime}$ & (30) \\
\hline 4395.477 & $>20.406$ & $i^{\prime}$ & (30) \\
\hline \multicolumn{4}{|c|}{ M31N 2007-11b } \\
\hline 4418.534 & $19.830 \pm 0.027$ & $B$ & $(30)$ \\
\hline 4420.510 & $19.829 \pm 0.026$ & $B$ & (30) \\
\hline 4421.434 & $19.753 \pm 0.023$ & $B$ & $(30)$ \\
\hline 4431.597 & $21.004 \pm 0.144$ & $B$ & (30) \\
\hline 4432.478 & $20.848 \pm 0.096$ & $B$ & (30) \\
\hline 4438.431 & $20.805 \pm 0.047$ & $B$ & (30) \\
\hline 4444.422 & $20.976 \pm 0.045$ & $B$ & (30) \\
\hline 4419.872 & $19.933 \pm 0.134$ & $B$ & $(31)$ \\
\hline 4421.846 & $19.742 \pm 0.080$ & $B$ & $(31)$ \\
\hline 4422.762 & $20.191 \pm 0.100$ & $B$ & (31) \\
\hline 4427.856 & $>19.946$ & $B$ & (31) \\
\hline 4418.537 & $19.662 \pm 0.026$ & $V$ & $(30)$ \\
\hline 4420.513 & $19.628 \pm 0.024$ & $V$ & $(30)$ \\
\hline 4421.437 & $19.509 \pm 0.021$ & $V$ & $(30)$ \\
\hline 4431.602 & $20.391 \pm 0.075$ & $V$ & (30) \\
\hline 4432.481 & $20.557 \pm 0.070$ & $V$ & $(30)$ \\
\hline 4438.434 & $20.581 \pm 0.043$ & $V$ & (30) \\
\hline 4441.433 & $19.966 \pm 0.030$ & $V$ & (30) \\
\hline 4444.424 & $20.691 \pm 0.039$ & $V$ & (30) \\
\hline 4419.874 & $19.687 \pm 0.064$ & $V$ & $(31)$ \\
\hline 4421.849 & $19.551 \pm 0.051$ & $V$ & (31) \\
\hline 4422.765 & $19.802 \pm 0.056$ & $V$ & (31) \\
\hline 4427.859 & $20.272 \pm 0.210$ & $V$ & $(31)$ \\
\hline 3771.346 & $>21.0$ & $R$ & $(21)$ \\
\hline 4415.446 & $18.3 \pm 0.15$ & $R$ & $(1)$ \\
\hline 4416.223 & $18.2 \pm 0.15$ & $R$ & (1) \\
\hline 4453.263 & $19.9 \pm 0.25$ & $R$ & (1) \\
\hline 4418.540 & $19.246 \pm 0.028$ & $i^{\prime}$ & $(30)$ \\
\hline 4420.516 & $19.159 \pm 0.026$ & $i^{\prime}$ & (30) \\
\hline
\end{tabular}


Table 3-Continued

\begin{tabular}{|c|c|c|c|}
\hline $\begin{array}{c}\text { JD } \\
(2,450,000+)\end{array}$ & Mag & Filter & Notes $^{a}$ \\
\hline 4421.440 & $19.063 \pm 0.045$ & $i^{\prime}$ & $(30)$ \\
\hline 4431.607 & $19.858 \pm 0.041$ & $i^{\prime}$ & (30) \\
\hline 4432.484 & $19.669 \pm 0.033$ & $i^{\prime}$ & (30) \\
\hline 4438.437 & $19.735 \pm 0.033$ & $i^{\prime}$ & (30) \\
\hline 4441.436 & $19.366 \pm 0.030$ & $i^{\prime}$ & (30) \\
\hline 4444.427 & $20.039 \pm 0.032$ & $i^{\prime}$ & (30) \\
\hline 4419.877 & $19.124 \pm 0.041$ & $i^{\prime}$ & $(31)$ \\
\hline 4421.852 & $19.054 \pm 0.036$ & $i^{\prime}$ & (31) \\
\hline 4422.768 & $19.355 \pm 0.041$ & $i^{\prime}$ & (31) \\
\hline 4427.862 & $19.607 \pm 0.092$ & $i^{\prime}$ & (31) \\
\hline 4428.758 & $19.126 \pm 0.093$ & $i^{\prime}$ & (31) \\
\hline 4430.869 & $19.553 \pm 0.060$ & $i^{\prime}$ & (31) \\
\hline \multirow[t]{2}{*}{4436.765} & $19.807 \pm 0.061$ & $i^{\prime}$ & (31) \\
\hline & M31N 2007-11c & & \\
\hline 4419.883 & $16.642 \pm 0.067$ & $B$ & $(31)$ \\
\hline 4420.536 & $16.736 \pm 0.013$ & $B$ & (30) \\
\hline 4421.447 & $16.718 \pm 0.012$ & $B$ & (30) \\
\hline 4421.858 & $16.985 \pm 0.027$ & $B$ & (31) \\
\hline 4422.774 & $17.058 \pm 0.024$ & $B$ & (31) \\
\hline 4427.868 & $>18.311$ & $B$ & $(31)$ \\
\hline 4430.875 & $>18.536$ & $B$ & (31) \\
\hline 4432.385 & $18.524 \pm 0.020$ & $B$ & $(30)$ \\
\hline 4436.771 & $19.117 \pm 0.094$ & $B$ & (30) \\
\hline 4438.418 & $19.184 \pm 0.035$ & $B$ & (30) \\
\hline 4441.397 & $19.423 \pm 0.046$ & $B$ & $(30)$ \\
\hline 4443.534 & $>20.583$ & $B$ & (30) \\
\hline 4449.347 & $20.019 \pm 0.052$ & $B$ & (30) \\
\hline 4419.886 & $16.651 \pm 0.016$ & $V$ & (31) \\
\hline 4420.539 & $16.926 \pm 0.014$ & $V$ & (30) \\
\hline 4421.450 & $17.028 \pm 0.011$ & $V$ & (30) \\
\hline 4421.860 & $17.165 \pm 0.019$ & $V$ & (31) \\
\hline 4422.776 & $17.327 \pm 0.019$ & $V$ & (31) \\
\hline 4427.870 & $>17.968$ & $V$ & (31) \\
\hline 4428.767 & $>18.804$ & $V$ & (31) \\
\hline 4429.766 & $18.521 \pm 0.054$ & $V$ & (31) \\
\hline 4430.878 & $18.616 \pm 0.127$ & $V$ & (31) \\
\hline 4432.388 & $18.891 \pm 0.028$ & $V$ & (30) \\
\hline 4436.773 & $19.266 \pm 0.091$ & $V$ & $(31)$ \\
\hline 4438.421 & $19.482 \pm 0.064$ & $V$ & $(30)$ \\
\hline 4441.400 & $19.890 \pm 0.079$ & $V$ & (30) \\
\hline 4443.537 & $>19.933$ & $V$ & (30) \\
\hline
\end{tabular}


Table 3-Continued

\begin{tabular}{|c|c|c|c|}
\hline $\begin{array}{c}\text { JD } \\
(2,450,000+)\end{array}$ & Mag & Filter & Notes $^{\mathrm{a}}$ \\
\hline 4449.350 & $20.334 \pm 0.107$ & $V$ & $(30)$ \\
\hline 3771.346 & $>21.5$ & $R$ & $(21)$ \\
\hline 4415.475 & $>20.1$ & $R$ & (1) \\
\hline 4416.387 & $19.7 \pm 0.25$ & $R$ & (1) \\
\hline 4417.547 & $17.8 \pm 0.2$ & $R$ & (1) \\
\hline 4445.234 & $19.3: \pm 0.4$ & $R$ & (1) \\
\hline 4419.888 & $17.173 \pm 0.082$ & $i^{\prime}$ & $(31)$ \\
\hline 4420.542 & $17.467 \pm 0.081$ & $i^{\prime}$ & (30) \\
\hline 4421.453 & $17.592 \pm 0.081$ & $i^{\prime}$ & (30) \\
\hline 4421.863 & $17.710 \pm 0.084$ & $i^{\prime}$ & (31) \\
\hline 4422.779 & $17.848 \pm 0.083$ & $i^{\prime}$ & (31) \\
\hline 4427.873 & $>18.935$ & $i^{\prime}$ & $(31)$ \\
\hline 4429.769 & $19.961 \pm 0.154$ & $i^{\prime}$ & (31) \\
\hline 4432.391 & $19.539 \pm 0.107$ & $i^{\prime}$ & (30) \\
\hline 4438.424 & $19.955 \pm 0.171$ & $i^{\prime}$ & (30) \\
\hline 4443.540 & $>18.842$ & $i^{\prime}$ & (30) \\
\hline 4449.352 & $>20.690$ & $i^{\prime}$ & (30) \\
\hline \multicolumn{4}{|c|}{ M31N 2007-11d } \\
\hline 4427.880 & $16.460 \pm 0.030$ & $B$ & $(30)$ \\
\hline 4428.780 & $16.520 \pm 0.040$ & $B$ & (30) \\
\hline 4429.770 & $16.560 \pm 0.030$ & $B$ & (30) \\
\hline 4432.470 & $16.639 \pm 0.027$ & $B$ & (31) \\
\hline 4436.580 & $17.558 \pm 0.028$ & $B$ & (31) \\
\hline 4436.780 & $17.820 \pm 0.070$ & $B$ & (30) \\
\hline 4439.370 & $20.220 \pm 0.040$ & $B$ & $(31)$ \\
\hline 4443.330 & $21.772 \pm 0.029$ & $B$ & $(31)$ \\
\hline 4427.880 & $16.071 \pm 0.024$ & $V$ & $(30)$ \\
\hline 4428.780 & $16.236 \pm 0.026$ & $V$ & (30) \\
\hline 4429.780 & $16.334 \pm 0.024$ & $V$ & $(30)$ \\
\hline 4432.470 & $16.603 \pm 0.023$ & $V$ & (31) \\
\hline 4436.590 & $17.563 \pm 0.031$ & $V$ & (31) \\
\hline 4436.780 & $17.703 \pm 0.043$ & $V$ & (30) \\
\hline 4439.370 & $20.039 \pm 0.036$ & $V$ & (31) \\
\hline 4443.340 & $21.443 \pm 0.133$ & $V$ & (31) \\
\hline 4427.880 & $15.780 \pm 0.010$ & $i^{\prime}$ & $(30)$ \\
\hline 4428.780 & $15.890 \pm 0.030$ & $i^{\prime}$ & (30) \\
\hline 4429.780 & $16.040 \pm 0.010$ & $i^{\prime}$ & (30) \\
\hline 4432.470 & $16.310 \pm 0.010$ & $i^{\prime}$ & (31) \\
\hline 4436.590 & $17.070 \pm 0.010$ & $i^{\prime}$ & $(31)$ \\
\hline
\end{tabular}


Table 3-Continued

\begin{tabular}{cccc}
\hline \hline JD & Mag & Filter & Notes $^{\mathrm{a}}$ \\
\hline $2,450,000+)$ & $17.270 \pm 0.030$ & $i^{\prime}$ & $(30)$ \\
4436.790 & $18.710 \pm 0.020$ & $i^{\prime}$ & $(31)$ \\
4439.380 & $20.270 \pm 0.070$ & $i^{\prime}$ & $(31)$ \\
4443.340 & $20.880 \pm 0.090$ & $i^{\prime}$ & $(31)$ \\
4449.340 & M31N $2007-12 \mathrm{a}$ & & \\
\hline & $18.002 \pm 0.023$ & $B$ & $(30)$ \\
\hline 4449.415 & $17.709 \pm 0.023$ & $B$ & $(30)$ \\
4451.434 & $17.872 \pm 0.032$ & $B$ & $(30)$ \\
4455.392 & $18.069 \pm 0.024$ & $B$ & $(30)$ \\
4459.390 & $18.114 \pm 0.023$ & $B$ & $(30)$ \\
4460.344 & $18.275 \pm 0.024$ & $B$ & $(30)$ \\
4461.386 & $18.410 \pm 0.024$ & $B$ & $(30)$ \\
4463.473 & $18.664 \pm 0.024$ & $B$ & $(30)$ \\
4466.335 & $18.790 \pm 0.025$ & $B$ & $(30)$ \\
4467.367 & $18.864 \pm 0.024$ & $B$ & $(30)$ \\
4468.388 & $19.050 \pm 0.025$ & $B$ & $(30)$ \\
4469.432 & $19.135 \pm 0.026$ & $B$ & $(30)$ \\
4471.343 & $19.533 \pm 0.067$ & $B$ & $(31)$ \\
4473.862 & $19.253 \pm 0.026$ & $B$ & $(30)$ \\
4474.413 & $19.402 \pm 0.056$ & $B$ & $(31)$ \\
4474.732 & $19.338 \pm 0.101$ & $B$ & $(31)$ \\
4474.741 & $19.374 \pm 0.314 \pm 0.069$ & $V$ & $(31)$ \\
4449.418 & $17.817 \pm 0.013$ & $V$ & $(30)$ \\
4451.437 & $17.601 \pm 0.013$ & $V$ & $(30)$ \\
4455.395 & $17.803 \pm 0.013$ & $V$ & $(30)$ \\
4459.393 & $18.020 \pm 0.014$ & $V$ & $(30)$ \\
4460.347 & $18.079 \pm 0.014$ & $V$ & $(30)$ \\
4461.389 & $18.246 \pm 0.014$ & $V$ & $(30)$ \\
4463.476 & $18.436 \pm 0.015$ & $V$ & $(30)$ \\
4466.338 & $18.647 \pm 0.015$ & $V$ & $(30)$ \\
4467.370 & $18.789 \pm 0.016$ & $V$ & $(30)$ \\
4468.391 & $18.842 \pm 0.016$ & $V$ & $(30)$ \\
4469.435 & $19.013 \pm 0.017$ & $V$ & $(30)$ \\
4471.346 & $19.093 \pm 0.018$ & $V$ & $(30)$ \\
4473.865 & $19.475 \pm 0.046$ & $V$ & $(31)$ \\
4474.416 & $19.255 \pm 0.018$ & $V$ & $(30)$ \\
4474.734 & $19.349 \pm 0.049$ & $V$ & $(31)$ \\
4474.744 & $i^{\prime}$ & & $(30)$ \\
4449.421 & & &
\end{tabular}


Table 3-Continued

\begin{tabular}{|c|c|c|c|}
\hline $\begin{array}{c}\text { JD } \\
(2,450,000+)\end{array}$ & Mag & Filter & Notes $^{\mathrm{a}}$ \\
\hline 4460.350 & $17.778 \pm 0.012$ & $i^{\prime}$ & $(30)$ \\
\hline 4461.392 & $17.917 \pm 0.013$ & $i^{\prime}$ & (30) \\
\hline 4463.479 & $18.045 \pm 0.013$ & $i^{\prime}$ & (30) \\
\hline 4466.341 & $18.289 \pm 0.013$ & $i^{\prime}$ & (30) \\
\hline 4467.373 & $18.363 \pm 0.014$ & $i^{\prime}$ & (30) \\
\hline 4468.394 & $18.404 \pm 0.014$ & $i^{\prime}$ & (30) \\
\hline 4469.437 & $18.540 \pm 0.015$ & $i^{\prime}$ & (30) \\
\hline 4471.349 & $18.621 \pm 0.016$ & $i^{\prime}$ & (30) \\
\hline 4473.868 & $18.741 \pm 0.027$ & $i^{\prime}$ & (31) \\
\hline 4474.419 & $18.761 \pm 0.016$ & $i^{\prime}$ & (30) \\
\hline 4474.747 & $18.831 \pm 0.043$ & $i^{\prime}$ & $(31)$ \\
\hline \multicolumn{4}{|c|}{ M31N 2007-12b } \\
\hline 4449.440 & $19.190 \pm 0.050$ & $B$ & $(30)$ \\
\hline 4452.350 & $19.750 \pm 0.050$ & $B$ & (30) \\
\hline 4455.400 & $20.270 \pm 0.090$ & $B$ & (30) \\
\hline 4459.400 & $20.880 \pm 0.110$ & $B$ & (30) \\
\hline 4460.390 & $21.120 \pm 0.110$ & $B$ & (30) \\
\hline 4461.510 & $>20.54$ & $B$ & (30) \\
\hline 4464.520 & $21.590 \pm 0.050$ & $B$ & (30) \\
\hline 4467.350 & $>21.4$ & $B$ & (30) \\
\hline 4468.400 & $>21.01$ & $B$ & (30) \\
\hline 4469.460 & $>21.63$ & $B$ & (30) \\
\hline 4470.440 & $>21.78$ & $B$ & (30) \\
\hline 4472.480 & $>21.73$ & $B$ & (30) \\
\hline 4449.450 & $19.270 \pm 0.040$ & $V$ & (30) \\
\hline 4452.350 & $19.820 \pm 0.040$ & $V$ & (30) \\
\hline 4455.410 & $20.430 \pm 0.090$ & $V$ & (30) \\
\hline 4459.410 & $20.670 \pm 0.090$ & $V$ & (30) \\
\hline 4460.390 & $>21.11$ & $V$ & (30) \\
\hline 4461.510 & $>20.74$ & $V$ & (30) \\
\hline 4464.520 & $>20.55$ & $V$ & (30) \\
\hline 4467.350 & $>20.88$ & $V$ & (30) \\
\hline 4468.400 & $>20.97$ & $V$ & (30) \\
\hline 4469.470 & $>20.62$ & $V$ & (30) \\
\hline 4470.450 & $>20.96$ & $V$ & (30) \\
\hline 4472.480 & $>21.09$ & $V$ & (30) \\
\hline 3771.346 & $>21.5$ & $R$ & (21) \\
\hline 4417.547 & $>19.1$ & $R$ & (1) \\
\hline 4445.234 & $17.0 \pm 0.1$ & $R$ & (1) \\
\hline 4450.392 & $18.6 \pm 0.25$ & $R$ & (1) \\
\hline 4450.402 & $18.7 \pm 0.25$ & $R$ & (1) \\
\hline
\end{tabular}


Table 3-Continued

\begin{tabular}{|c|c|c|c|}
\hline $\begin{array}{c}\mathrm{JD} \\
(2,450,000+)\end{array}$ & Mag & Filter & Notes $^{\mathrm{a}}$ \\
\hline 4453.250 & $19.4 \pm 0.25$ & $R$ & (1) \\
\hline 4449.450 & $18.930 \pm 0.040$ & $i^{\prime}$ & (30) \\
\hline 4452.360 & $19.350 \pm 0.040$ & $i^{\prime}$ & (30) \\
\hline 4455.410 & $>19.65$ & $i^{\prime}$ & (30) \\
\hline 4459.410 & $>19.58$ & $i^{\prime}$ & (30) \\
\hline 4460.390 & $>19.64$ & $i^{\prime}$ & (30) \\
\hline \multicolumn{4}{|c|}{$\mathrm{M} 31 \mathrm{~N} 2007-12 \mathrm{~d}$} \\
\hline 3771.346 & $>21.5$ & $R$ & $(21)$ \\
\hline 4453.238 & $17.8 \pm 0.2$ & $R$ & (1) \\
\hline \multicolumn{4}{|c|}{ M31N 2008-05c } \\
\hline 4528.246 & $>19.6$ & $R$ & (1) \\
\hline 4601.558 & $>19.8$ & $R$ & (28) \\
\hline 4617.523 & $17.6 \pm 0.2$ & $R$ & $(1)$ \\
\hline 4617.549 & $17.5 \pm 0.2$ & $R$ & (1) \\
\hline 4619.529 & $17.2 \pm 0.1$ & $R$ & (1) \\
\hline 4619.554 & $17.2 \pm 0.1$ & $R$ & (1) \\
\hline 4620.543 & $17.3 \pm 0.1$ & $R$ & (1) \\
\hline 4628.541 & $18.1 \pm 0.2$ & $R$ & (3) \\
\hline 4645.550 & $19.3 \pm 0.2$ & $R$ & (1) \\
\hline 4646.535 & $19.0 \pm 0.2$ & $R$ & (1) \\
\hline 4647.492 & $19.0 \pm 0.2$ & $R$ & (1) \\
\hline 4648.557 & $19.0 \pm 0.2$ & $R$ & (1) \\
\hline 4655.567 & $19.6 \pm 0.25$ & $R$ & (3) \\
\hline 4675.581 & $19.3 \pm 0.2$ & $R$ & (3) \\
\hline 4677.432 & $19.4 \pm 0.3$ & $R$ & (1) \\
\hline 4678.562 & $19.6 \pm 0.25$ & $R$ & (1) \\
\hline 4679.551 & $19.5 \pm 0.25$ & $R$ & (1) \\
\hline 4682.379 & $19.6 \pm 0.3$ & $R$ & (1) \\
\hline 4682.574 & $19.6 \pm 0.2$ & $R$ & (1) \\
\hline 4683.562 & $19.8 \pm 0.3$ & $R$ & (1) \\
\hline 4685.548 & $20.0 \pm 0.3$ & $R$ & (1) \\
\hline 4692.616 & $>19.7$ & $R$ & $(28)$ \\
\hline 4697.573 & $20.3 \pm 0.3$ & $R$ & (3) \\
\hline 4706.362 & $19.6 \pm 0.3$ & $R$ & (1) \\
\hline 4712.608 & $>20.1$ & $R$ & (1) \\
\hline \multicolumn{4}{|c|}{ M31N 2008-06b } \\
\hline 4505.226 & $>20.0$ & $R$ & (1) \\
\hline 4620.542 & $>20.3$ & $R$ & (1) \\
\hline
\end{tabular}


Table 3-Continued

\begin{tabular}{|c|c|c|c|}
\hline $\begin{array}{c}\text { JD } \\
(2,450,000+)\end{array}$ & Mag & Filter & Notes $^{\mathrm{a}}$ \\
\hline 4628.541 & $>20.2$ & $R$ & (3) \\
\hline 4644.498 & $16.0 \pm 0.1$ & $R$ & (5) \\
\hline 4645.489 & $16.44 \pm 0.05$ & $R$ & (1) \\
\hline 4645.529 & $16.41 \pm 0.05$ & $R$ & (1) \\
\hline 4645.550 & $16.41 \pm 0.05$ & $R$ & (1) \\
\hline 4646.535 & $16.54 \pm 0.05$ & $R$ & (1) \\
\hline 4647.492 & $16.62 \pm 0.05$ & $R$ & (1) \\
\hline 4648.557 & $16.71 \pm 0.05$ & $R$ & (1) \\
\hline 4652.560 & $17.26 \pm 0.1$ & $R$ & (3) \\
\hline 4655.567 & $17.45 \pm 0.1$ & $R$ & (3) \\
\hline 4675.581 & $18.9 \pm 0.15$ & $R$ & (3) \\
\hline 4677.432 & $18.75 \pm 0.2$ & $R$ & (1) \\
\hline 4678.562 & $18.8 \pm 0.15$ & $R$ & (1) \\
\hline 4679.551 & $19.0 \pm 0.15$ & $R$ & (1) \\
\hline 4681.370 & $19.1 \pm 0.25$ & $R$ & (1) \\
\hline 4682.379 & $19.1 \pm 0.2$ & $R$ & (1) \\
\hline 4682.574 & $19.0 \pm 0.15$ & $R$ & (1) \\
\hline 4683.562 & $19.1 \pm 0.2$ & $R$ & (1) \\
\hline 4685.548 & $19.4 \pm 0.2$ & $R$ & (1) \\
\hline 4692.616 & $>19.7$ & $R$ & $(28)$ \\
\hline 4697.573 & $20.4 \pm 0.3$ & $R$ & (3) \\
\hline 4706.362 & $>20.4$ & $R$ & (1) \\
\hline \multicolumn{4}{|c|}{ M31N 2008-07a } \\
\hline 5095.981 & $21.4 \pm 0.4 \mathrm{~V}(13)$ & & \\
\hline 4505.226 & $>19.7$ & $R$ & (1) \\
\hline 4617.523 & $>19.0$ & $R$ & (1) \\
\hline 4619.529 & $19.0 \pm 0.3$ & $R$ & (1) \\
\hline 4620.542 & $18.7 \pm 0.25$ & $R$ & (1) \\
\hline 4628.541 & $19.0 \pm 0.3$ & $R$ & (3) \\
\hline 4645.488 & $19.1 \pm 0.3$ & $R$ & (1) \\
\hline 4646.535 & $19.2 \pm 0.25$ & $R$ & (1) \\
\hline 4647.492 & $18.8 \pm 0.2$ & $R$ & (1) \\
\hline 4648.557 & $19.3 \pm 0.25$ & $R$ & (1) \\
\hline 4655.567 & $19.1 \pm 0.25$ & $R$ & (3) \\
\hline 4675.581 & $19.0 \pm 0.2$ & $R$ & (3) \\
\hline 4677.432 & $18.8 \pm 0.2$ & $R$ & (1) \\
\hline 4678.562 & $18.8 \pm 0.2$ & $R$ & (1) \\
\hline 4679.551 & $18.9 \pm 0.2$ & $R$ & (1) \\
\hline 4681.370 & $19.3 \pm 0.3$ & $R$ & (1) \\
\hline 4682.379 & $19.1 \pm 0.25$ & $R$ & (1) \\
\hline 4682.574 & $19.0 \pm 0.15$ & $R$ & (1) \\
\hline 4683.562 & $19.0 \pm 0.2$ & $R$ & (1) \\
\hline
\end{tabular}


Table 3-Continued

\begin{tabular}{|c|c|c|c|}
\hline $\begin{array}{c}\text { JD } \\
(2,450,000+)\end{array}$ & Mag & Filter & Notes $^{\mathrm{a}}$ \\
\hline 4684.525 & $19.5 \pm 0.3$ & $R$ & (1) \\
\hline 4685.548 & $18.75 \pm 0.1$ & $R$ & (1) \\
\hline 4692.616 & $18.9 \pm 0.2$ & $R$ & $(28)$ \\
\hline 4697.573 & $19.0 \pm 0.15$ & $R$ & (3) \\
\hline 4706.362 & $19.1 \pm 0.2$ & $R$ & (1) \\
\hline 4708.387 & $18.7 \pm 0.25$ & $R$ & (1) \\
\hline 4709.613 & $19.3 \pm 0.25$ & $R$ & (1) \\
\hline 4710.589 & $19.2 \pm 0.35$ & $R$ & (1) \\
\hline 4711.310 & $18.9 \pm 0.25$ & $R$ & (1) \\
\hline 4712.291 & $19.0 \pm 0.25$ & $R$ & (1) \\
\hline 4712.337 & $18.8 \pm 0.15$ & $R$ & (1) \\
\hline 4712.608 & $18.85 \pm 0.15$ & $R$ & (1) \\
\hline 4713.343 & $19.0 \pm 0.2$ & $R$ & (1) \\
\hline 4715.330 & $18.8 \pm 0.15$ & $R$ & (1) \\
\hline 4716.334 & $18.9 \pm 0.25$ & $R$ & $(1)$ \\
\hline 4718.378 & $19.3 \pm 0.3$ & $R$ & (3) \\
\hline 4719.299 & $19.2 \pm 0.3$ & $R$ & (3) \\
\hline 4738.221 & $18.85 \pm 0.15$ & $R$ & (3) \\
\hline 4744.604 & $19.1 \pm 0.15$ & $R$ & (1) \\
\hline 4745.257 & $19.1 \pm 0.2$ & $R$ & (1) \\
\hline 4748.472 & $19.1 \pm 0.2$ & $R$ & $(7)$ \\
\hline 4748.485 & $19.2 \pm 0.25$ & $R$ & $(7)$ \\
\hline 4754.400 & $19.0 \pm 0.2$ & $R$ & (3) \\
\hline 4760.628 & $19.2 \pm 0.2$ & $R$ & (3) \\
\hline 4763.219 & $19.2 \pm 0.3$ & $R$ & (1) \\
\hline 4765.545 & $19.1 \pm 0.25$ & $R$ & (1) \\
\hline 4772.448 & $19.4 \pm 0.25$ & $R$ & (1) \\
\hline 4774.545 & $19.3 \pm 0.3$ & $R$ & (1) \\
\hline 4776.228 & $19.6 \pm 0.25$ & $R$ & (1) \\
\hline 4777.215 & $19.6 \pm 0.3$ & $R$ & $(1)$ \\
\hline 4779.283 & $19.4 \pm 0.25$ & $R$ & (1) \\
\hline 4779.318 & $19.5 \pm 0.3$ & $R$ & (1) \\
\hline 4800.301 & $19.3 \pm 0.3$ & $R$ & (1) \\
\hline 4801.226 & $18.9: \pm 0.4$ & $R$ & $(1)$ \\
\hline 4809.285 & $19.0: \pm 0.4$ & $R$ & (1) \\
\hline 4982.972 & $>20.4$ & $R$ & (9) \\
\hline 5095.968 & $20.9 \pm 0.25$ & $R$ & (13) \\
\hline \multicolumn{4}{|c|}{ M31N 2008-07b } \\
\hline 4505.226 & $>19.7$ & $R$ & (1) \\
\hline 4655.567 & $>20.6$ & $R$ & (3) \\
\hline 4675.581 & $18.4 \pm 0.1$ & $R$ & (3) \\
\hline 4677.432 & $18.8 \pm 0.2$ & $R$ & (1) \\
\hline 4677.448 & $18.6 \pm 0.2$ & $R$ & (1) \\
\hline
\end{tabular}


Table 3-Continued

\begin{tabular}{|c|c|c|c|}
\hline $\begin{array}{c}\text { JD } \\
(2,450,000+)\end{array}$ & Mag & Filter & Notes \\
\hline 4678.562 & $18.7 \pm 0.15$ & $R$ & (1) \\
\hline 4679.551 & $18.8 \pm 0.15$ & $R$ & (1) \\
\hline 4681.370 & $19.3 \pm 0.25$ & $R$ & (1) \\
\hline 4682.379 & $19.6 \pm 0.25$ & $R$ & (1) \\
\hline 4682.550 & $19.3 \pm 0.2$ & $R$ & (1) \\
\hline 4682.574 & $19.4 \pm 0.15$ & $R$ & (1) \\
\hline 4683.562 & $19.3 \pm 0.15$ & $R$ & (1) \\
\hline 4684.525 & $19.5 \pm 0.3$ & $R$ & (1) \\
\hline 4685.548 & $19.6 \pm 0.15$ & $R$ & (1) \\
\hline 4685.564 & $19.5 \pm 0.15$ & $R$ & (1) \\
\hline 4685.577 & $19.4 \pm 0.15$ & $R$ & (1) \\
\hline 4692.616 & $19.3 \pm 0.25$ & $R$ & $(28)$ \\
\hline 4697.573 & $20.0 \pm 0.25$ & $R$ & $(3)$ \\
\hline 4706.362 & $20.7 \pm 0.3$ & $R$ & (1) \\
\hline 4708.424 & $20.3 \pm 0.3$ & $R$ & (1) \\
\hline 4710.323 & $>20.2$ & $R$ & (1) \\
\hline 4711.335 & $20.6 \pm 0.4$ & $R$ & (1) \\
\hline 4712.608 & $20.3 \pm 0.25$ & $R$ & (1) \\
\hline 4713.343 & $20.2 \pm 0.35$ & $R$ & (1) \\
\hline 4715.330 & $20.5 \pm 0.3$ & $R$ & (1) \\
\hline \multicolumn{4}{|c|}{ M31N 2008-08a } \\
\hline 4685.548 & $>20.5$ & $R$ & (1) \\
\hline 4692.616 & $16.7 \pm 0.1$ & $R$ & $(28)$ \\
\hline 4697.573 & $17.7 \pm 0.1$ & $R$ & $(3)$ \\
\hline 4706.362 & $18.3 \pm 0.15$ & $R$ & (1) \\
\hline 4706.627 & $18.2 \pm 0.2$ & $R$ & (1) \\
\hline 4708.387 & $18.4 \pm 0.25$ & $R$ & (1) \\
\hline 4709.613 & $18.8 \pm 0.25$ & $R$ & (1) \\
\hline 4710.589 & $19.0 \pm 0.3$ & $R$ & (1) \\
\hline 4711.310 & $18.4 \pm 0.25$ & $R$ & (1) \\
\hline 4712.291 & $19.3 \pm 0.35$ & $R$ & (1) \\
\hline 4712.608 & $19.0 \pm 0.2$ & $R$ & (1) \\
\hline 4713.343 & $18.6 \pm 0.25$ & $R$ & (1) \\
\hline 4715.330 & $18.6 \pm 0.25$ & $R$ & (1) \\
\hline \multicolumn{4}{|c|}{ M31N 2008-08b } \\
\hline 4685.548 & $>20.5$ & $R$ & (1) \\
\hline 4692.616 & $17.8 \pm 0.1$ & $R$ & $(28)$ \\
\hline 4697.573 & $19.3 \pm 0.2$ & $R$ & $(3)$ \\
\hline \multicolumn{4}{|c|}{ M31N 2008-09a } \\
\hline
\end{tabular}


Table 3-Continued

\begin{tabular}{cccc}
\hline \hline JD & & & \\
$(2,450,000+)$ & Mag & Filter & Notes $^{\mathrm{a}}$ \\
\hline 4744.340 & $18.4 \pm 0.2$ & $R$ & $(1)$ \\
\hline & M31N $2008-10 \mathrm{a}$ & & \\
\hline 4778.655 & $19.884 \pm 0.171$ & $B$ & $(30)$ \\
4778.727 & $19.477 \pm 0.071$ & $B$ & $(31)$ \\
4779.967 & $>20.168$ & $B$ & $(31)$ \\
4779.637 & $20.080 \pm 0.195$ & $B$ & $(30)$ \\
4780.463 & $20.093 \pm 0.078$ & $B$ & $(30)$ \\
4781.491 & $>18.797$ & $B$ & $(30)$ \\
4782.449 & $19.515 \pm 0.060$ & $B$ & $(30)$ \\
4783.360 & $>19.960$ & $B$ & $(30)$ \\
4785.451 & $19.651 \pm 0.042$ & $B$ & $(30)$ \\
4786.404 & $>19.649$ & $B$ & $(30)$ \\
4787.479 & $19.402 \pm 0.033$ & $B$ & $(30)$ \\
4788.455 & $19.650 \pm 0.035$ & $B$ & $(30)$ \\
4789.403 & $19.683 \pm 0.038$ & $B$ & $(30)$ \\
& & & \\
4778.658 & $19.337 \pm 0.032$ & $V$ & $(30)$ \\
4778.732 & $19.964 \pm 0.094$ & $V$ & $(31)$ \\
4779.641 & $19.764 \pm 0.036$ & $V$ & $(30)$ \\
4779.972 & $19.781 \pm 0.103$ & $V$ & $(31)$ \\
4780.466 & $20.100 \pm 0.070$ & $V$ & $(30)$ \\
4780.900 & $18.880 \pm 0.582$ & $V$ & $(31)$ \\
4781.495 & $>19.338$ & $V$ & $(30)$ \\
4782.453 & $19.254 \pm 0.049$ & $V$ & $(30)$ \\
4783.364 & $19.543 \pm 0.068$ & $V$ & $(30)$ \\
4785.456 & $19.456 \pm 0.040$ & $V$ & $(30)$ \\
4786.407 & $19.412 \pm 0.038$ & $V$ & $(30)$ \\
4787.483 & $19.194 \pm 0.026$ & $V$ & $(30)$ \\
4788.458 & $19.418 \pm 0.026$ & $V$ & $(30)$ \\
4789.406 & $19.444 \pm 0.026$ & $V$ & $(30)$ \\
& & & \\
4775.921 & $17.756 \pm 0.015$ & $r^{\prime}$ & $(31)$ \\
4777.861 & $17.975 \pm 0.039$ & $r^{\prime}$ & $(31)$ \\
4778.644 & $18.156 \pm 0.043$ & $r^{\prime}$ & $(30)$ \\
4778.712 & $18.147 \pm 0.016$ & $r^{\prime}$ & $(31)$ \\
4779.626 & $18.578 \pm 0.130$ & $r^{\prime}$ & $(30)$ \\
4779.952 & $18.118 \pm 0.063$ & $r^{\prime}$ & $(31)$ \\
4780.452 & $18.374 \pm 0.021$ & $r^{\prime}$ & $(30)$ \\
4780.877 & $18.165 \pm 0.140$ & $r^{\prime}$ & $(31)$ \\
4781.480 & $17.973 \pm 0.079$ & $r^{\prime}$ & $(30)$ \\
4782.438 & $18.094 \pm 0.016$ & $r^{\prime}$ & $(30)$ \\
4783.349 & $18.101 \pm 0.087$ & $r^{\prime}$ & $(30)$ \\
4785.440 & $18.239 \pm 0.012$ & $r^{\prime}$ & $(30)$
\end{tabular}


Table 3-Continued

\begin{tabular}{clcc}
\hline \hline JD & & & \\
$(2,450,000+)$ & \multicolumn{1}{c}{ Mag } & Filter & Notes $^{\mathrm{a}}$ \\
\hline 4786.393 & $18.275 \pm 0.013$ & $r^{\prime}$ & $(30)$ \\
4787.468 & $18.192 \pm 0.010$ & $r^{\prime}$ & $(30)$ \\
4788.444 & $18.357 \pm 0.011$ & $r^{\prime}$ & $(30)$ \\
4789.392 & $18.354 \pm 0.012$ & $r^{\prime}$ & $(30)$ \\
\hline & M31N $2008-10 \mathrm{~b}$ & & \\
\hline 4716.407 & $>20.1$ & $R$ & $(1)$ \\
4748.479 & $19.8 \pm 0.35$ & $R$ & $(4)$ \\
4754.419 & $19.3 \pm 0.35$ & $R$ & $(3)$ \\
4763.218 & $18.1 \pm 0.15$ & $R$ & $(1)$ \\
4765.546 & $19.0 \pm 0.25$ & $R$ & $(1)$ \\
4772.448 & $19.3 \pm 0.25$ & $R$ & $(1)$ \\
4774.571 & $19.2 \pm 0.25$ & $R$ & $(1)$ \\
4776.228 & $18.8 \pm 0.15$ & $R$ & $(1)$ \\
4777.229 & $18.5 \pm 0.15$ & $R$ & $(1)$ \\
4779.283 & $19.0 \pm 0.2$ & $R$ & $(1)$ \\
4795.192 & $19.9 \pm 0.3$ & $V$ & $(1)$ \\
4798.431 & $18.9 \pm 0.2$ & $V$ & $(5)$ \\
4799.199 & $18.3 \pm 0.15$ & $R$ & $(1)$ \\
4800.301 & $18.0 \pm 0.15$ & $R$ & $(1)$ \\
4801.267 & $18.3 \pm 0.15$ & $R$ & $(1)$ \\
4809.285 & $18.2 \pm 0.15$ & $R$ & $(1)$ \\
4829.183 & $19.1 \pm 0.3$ & $R$ & $(1)$ \\
4760.472 & $18.098 \pm 0.018$ & $B$ & $(30)$ \\
4761.514 & $17.574 \pm 0.018$ & $B$ & $(30)$ \\
4764.549 & $19.451 \pm 0.055$ & $B$ & $(30)$ \\
4777.584 & $18.525 \pm 0.030$ & $B$ & $(30)$ \\
4778.441 & $19.065 \pm 0.053$ & $B$ & $(30)$ \\
4779.485 & $19.113 \pm 0.052$ & $B$ & $(30)$ \\
4780.429 & $19.399 \pm 0.128$ & $B$ & $(30)$ \\
4781.435 & $19.343 \pm 0.100$ & $B$ & $(30)$ \\
4782.461 & $>19.780$ & $B$ & $(30)$ \\
4783.401 & $>19.115$ & $B$ & $(30)$ \\
4785.495 & $18.619 \pm 0.036$ & $B$ & $(30)$ \\
4786.422 & $18.853 \pm 0.055$ & $B$ & $(30)$ \\
4787.370 & $19.123 \pm 0.036$ & $B$ & $(30)$ \\
4788.467 & $19.475 \pm 0.053$ & $B$ & $(30)$ \\
4789.418 & $18.839 \pm 0.033$ & $B$ & $(30)$ \\
4790.383 & $19.035 \pm 0.044$ & $B$ & $(30)$ \\
4760.793 & $17.956 \pm 0.019$ & $B$ & $(31)$ \\
4761.793 & $17.652 \pm 0.018$ & $B$ & $(31)$ \\
4762.894 & $>17.446$ & $B$ & $(31)$
\end{tabular}


Table 3-Continued

\begin{tabular}{|c|c|c|c|}
\hline $\begin{array}{c}\text { JD } \\
(2,450,000+)\end{array}$ & Mag & Filter & Notes $^{a}$ \\
\hline 4763.727 & $18.993 \pm 0.044$ & $B$ & $(31)$ \\
\hline 4763.740 & $19.117 \pm 0.035$ & $B$ & $(31)$ \\
\hline 4763.793 & $19.105 \pm 0.034$ & $B$ & $(31)$ \\
\hline 4764.919 & $>19.756$ & $B$ & $(31)$ \\
\hline 4771.889 & $19.609 \pm 0.042$ & $B$ & $(31)$ \\
\hline 4772.862 & $19.898 \pm 0.047$ & $B$ & $(31)$ \\
\hline 4773.835 & $19.706 \pm 0.039$ & $B$ & $(31)$ \\
\hline 4774.842 & $19.460 \pm 0.040$ & $B$ & $(31)$ \\
\hline 4777.843 & $18.774 \pm 0.032$ & $B$ & (31) \\
\hline 4779.922 & $19.266 \pm 0.063$ & $B$ & (31) \\
\hline 4780.835 & $>18.461$ & $B$ & (31) \\
\hline 4760.476 & $18.124 \pm 0.016$ & $V$ & (30) \\
\hline 4761.517 & $17.706 \pm 0.014$ & $V$ & (30) \\
\hline 4764.553 & $19.322 \pm 0.054$ & $V$ & (30) \\
\hline 4777.588 & $18.671 \pm 0.033$ & $V$ & (30) \\
\hline 4778.444 & $18.231 \pm 0.059$ & $V$ & (30) \\
\hline 4779.487 & $19.262 \pm 0.049$ & $V$ & (30) \\
\hline 4780.433 & $19.636 \pm 0.085$ & $V$ & (30) \\
\hline 4781.438 & $19.731 \pm 0.110$ & $V$ & (30) \\
\hline 4782.465 & $19.750 \pm 0.182$ & $V$ & (30) \\
\hline 4783.405 & $>19.558$ & $V$ & (30) \\
\hline 4785.499 & $18.815 \pm 0.030$ & $V$ & (30) \\
\hline 4786.426 & $18.808 \pm 0.039$ & $V$ & (30) \\
\hline 4787.374 & $19.126 \pm 0.041$ & $V$ & $(30)$ \\
\hline 4788.471 & $19.674 \pm 0.067$ & $V$ & (30) \\
\hline 4789.421 & $18.897 \pm 0.035$ & $V$ & $(30)$ \\
\hline 4790.387 & $19.030 \pm 0.038$ & $V$ & (30) \\
\hline 4760.796 & $17.953 \pm 0.016$ & $V$ & $(31)$ \\
\hline 4761.796 & $17.699 \pm 0.015$ & $V$ & $(31)$ \\
\hline 4762.895 & $17.881 \pm 0.057$ & $V$ & $(31)$ \\
\hline 4763.730 & $18.975 \pm 0.031$ & $V$ & $(31)$ \\
\hline 4763.743 & $18.993 \pm 0.032$ & $V$ & (31) \\
\hline 4763.796 & $19.040 \pm 0.033$ & $V$ & $(31)$ \\
\hline 4764.923 & $>18.132$ & $V$ & $(31)$ \\
\hline 4771.892 & $19.653 \pm 0.051$ & $V$ & (31) \\
\hline 4772.865 & $20.034 \pm 0.062$ & $V$ & (31) \\
\hline 4773.837 & $19.859 \pm 0.049$ & $V$ & $(31)$ \\
\hline 4774.845 & $19.608 \pm 0.042$ & $V$ & $(31)$ \\
\hline 4777.846 & $18.773 \pm 0.035$ & $V$ & $(31)$ \\
\hline 4779.925 & $19.055 \pm 0.072$ & $V$ & (31) \\
\hline 4780.838 & $>17.488$ & $V$ & $(31)$ \\
\hline 4760.478 & $18.308 \pm 0.014$ & $r^{\prime}$ & (30) \\
\hline
\end{tabular}


Table 3-Continued

\begin{tabular}{|c|c|c|c|}
\hline $\begin{array}{c}\text { JD } \\
(2,450,000+)\end{array}$ & Mag & Filter & Notes $^{\mathrm{a}}$ \\
\hline 4761.520 & $17.840 \pm 0.012$ & $r^{\prime}$ & $(30)$ \\
\hline 4764.556 & $19.033 \pm 0.043$ & $r^{\prime}$ & (30) \\
\hline 4777.591 & $18.631 \pm 0.030$ & $r^{\prime}$ & (30) \\
\hline 4778.448 & $19.213 \pm 0.046$ & $r^{\prime}$ & (30) \\
\hline 4779.492 & $19.124 \pm 0.037$ & $r^{\prime}$ & (30) \\
\hline 4780.437 & $19.298 \pm 0.068$ & $r^{\prime}$ & (30) \\
\hline 4781.442 & $>19.089$ & $r^{\prime}$ & (30) \\
\hline 4782.469 & $>19.158$ & $r^{\prime}$ & (30) \\
\hline 4783.409 & $>19.260$ & $r^{\prime}$ & $(30)$ \\
\hline 4785.503 & $18.917 \pm 0.026$ & $r^{\prime}$ & (30) \\
\hline 4786.430 & $18.876 \pm 0.420$ & $r^{\prime}$ & (30) \\
\hline 4787.378 & $19.119 \pm 0.038$ & $r^{\prime}$ & $(30)$ \\
\hline 4788.474 & $19.378 \pm 0.053$ & $r^{\prime}$ & (30) \\
\hline 4789.425 & $18.934 \pm 0.031$ & $r^{\prime}$ & (30) \\
\hline 4790.391 & $19.029 \pm 0.036$ & $r^{\prime}$ & (30) \\
\hline 4760.799 & $18.171 \pm 0.014$ & $r^{\prime}$ & $(31)$ \\
\hline 4761.799 & $17.885 \pm 0.012$ & $r^{\prime}$ & (31) \\
\hline 4762.898 & $18.104 \pm 0.057$ & $r^{\prime}$ & $(31)$ \\
\hline 4763.733 & $18.926 \pm 0.026$ & $r^{\prime}$ & (31) \\
\hline 4763.799 & $18.935 \pm 0.025$ & $r^{\prime}$ & (31) \\
\hline 4764.925 & $19.333 \pm 0.387$ & $r^{\prime}$ & (31) \\
\hline 4771.895 & $19.378 \pm 0.035$ & $r^{\prime}$ & (31) \\
\hline 4772.868 & $19.719 \pm 0.045$ & $r^{\prime}$ & (31) \\
\hline 4773.841 & $19.566 \pm 0.037$ & $r^{\prime}$ & (31) \\
\hline 4774.848 & $19.441 \pm 0.033$ & $r^{\prime}$ & (31) \\
\hline 4777.850 & $18.781 \pm 0.033$ & $r^{\prime}$ & (31) \\
\hline 4779.928 & $19.219 \pm 0.048$ & $r^{\prime}$ & (31) \\
\hline 4780.841 & $18.677 \pm 0.235$ & $r^{\prime}$ & $(31)$ \\
\hline \multicolumn{4}{|c|}{ M31N 2008-11a } \\
\hline 4778.400 & $19.259 \pm 0.050$ & $B$ & $(30)$ \\
\hline 4779.472 & $19.783 \pm 0.064$ & $B$ & (30) \\
\hline 4779.891 & $19.309 \pm 0.193$ & $B$ & (31) \\
\hline 4780.486 & $19.607 \pm 0.069$ & $B$ & $(30)$ \\
\hline 4780.907 & $20.742 \pm 0.220$ & $B$ & $(31)$ \\
\hline 4782.418 & $20.027 \pm 0.083$ & $B$ & (30) \\
\hline 4783.496 & $>20.753$ & $B$ & (30) \\
\hline 4785.529 & $20.306 \pm 0.107$ & $B$ & (30) \\
\hline 4786.490 & $>20.029$ & $B$ & (30) \\
\hline 4787.404 & $20.507 \pm 0.072$ & $B$ & (30) \\
\hline 4788.428 & $20.933 \pm 0.074$ & $B$ & $(30)$ \\
\hline 4789.503 & $20.936 \pm 0.220$ & $B$ & $(30)$ \\
\hline 4794.344 & $20.592 \pm 0.082$ & $B$ & (30) \\
\hline
\end{tabular}


Table 3-Continued

\begin{tabular}{|c|c|c|c|}
\hline $\begin{array}{c}\text { JD } \\
(2,450,000+)\end{array}$ & Mag & Filter & Notes $^{\mathrm{a}}$ \\
\hline 4795.478 & $>20.830$ & $B$ & (30) \\
\hline 4796.581 & $>20.911$ & $B$ & $(30)$ \\
\hline 4799.384 & $21.567 \pm 0.114$ & $B$ & (30) \\
\hline 4800.470 & $>21.775$ & $B$ & (30) \\
\hline 4802.396 & $>21.644$ & $B$ & (30) \\
\hline 4805.339 & $>21.701$ & $B$ & (30) \\
\hline 4805.395 & $>21.562$ & $B$ & (30) \\
\hline 4778.404 & $18.917 \pm 0.038$ & $V$ & $(30)$ \\
\hline 4779.476 & $19.266 \pm 0.060$ & $V$ & (30) \\
\hline 4779.896 & $19.164 \pm 0.057$ & $V$ & (31) \\
\hline 4780.489 & $19.219 \pm 0.044$ & $V$ & (30) \\
\hline 4780.912 & $>19.462$ & $V$ & (31) \\
\hline 4781.472 & $19.693 \pm 0.095$ & $V$ & (30) \\
\hline 4782.422 & $19.770 \pm 0.110$ & $V$ & (30) \\
\hline 4783.499 & $>19.457$ & $V$ & (30) \\
\hline 4785.532 & $20.260 \pm 0.092$ & $V$ & (30) \\
\hline 4786.494 & $>19.410$ & $V$ & (30) \\
\hline 4787.407 & $20.177 \pm 0.059$ & $V$ & (30) \\
\hline 4788.432 & $20.407 \pm 0.141$ & $V$ & $(30)$ \\
\hline 4789.508 & $20.515 \pm 0.211$ & $V$ & (30) \\
\hline 4794.348 & $21.413 \pm 0.310$ & $V$ & (30) \\
\hline 4795.482 & $20.900 \pm 0.186$ & $V$ & (30) \\
\hline 4796.585 & $>20.561$ & $V$ & (30) \\
\hline 4799.387 & $20.737 \pm 0.147$ & $V$ & (30) \\
\hline 4800.474 & $>21.387$ & $V$ & (30) \\
\hline 4800.498 & $>20.384$ & $V$ & (30) \\
\hline 4802.400 & $21.172 \pm 0.185$ & $V$ & (30) \\
\hline 4805.399 & $>21.106$ & $V$ & (30) \\
\hline 4744.340 & $>19.9$ & $R$ & (1) \\
\hline 4775.218 & $16.5 \pm 0.1$ & $R$ & (1) \\
\hline 4776.217 & $17.4 \pm 0.1$ & $R$ & (1) \\
\hline 4779.260 & $18.3 \pm 0.15$ & $R$ & (1) \\
\hline 4780.234 & $18.4 \pm 0.2$ & $R$ & (1) \\
\hline 4800.310 & $>19.5$ & $R$ & (1) \\
\hline 4778.389 & $17.920 \pm 0.011$ & $r^{\prime}$ & (30) \\
\hline 4779.461 & $18.289 \pm 0.016$ & $r^{\prime}$ & (30) \\
\hline 4779.901 & $18.407 \pm 0.059$ & $r^{\prime}$ & (31) \\
\hline 4780.475 & $18.502 \pm 0.020$ & $r^{\prime}$ & (30) \\
\hline 4780.917 & $18.528 \pm 0.116$ & $r^{\prime}$ & (31) \\
\hline 4782.407 & $18.890 \pm 0.025$ & $r^{\prime}$ & (30) \\
\hline 4783.485 & $18.935 \pm 0.160$ & $r^{\prime}$ & (30) \\
\hline 4785.518 & $19.310 \pm 0.025$ & $r^{\prime}$ & (30) \\
\hline
\end{tabular}


Table 3-Continued

\begin{tabular}{|c|c|c|c|}
\hline $\begin{array}{c}\text { JD } \\
(2,450,000+)\end{array}$ & Mag & Filter & Notes $^{\mathrm{a}}$ \\
\hline 4786.479 & $19.436 \pm 0.160$ & $r^{\prime}$ & $(30)$ \\
\hline 4787.393 & $19.683 \pm 0.031$ & $r^{\prime}$ & (30) \\
\hline 4788.418 & $19.881 \pm 0.027$ & $r^{\prime}$ & (30) \\
\hline 4789.492 & $20.063 \pm 0.035$ & $r^{\prime}$ & $(30)$ \\
\hline 4790.412 & $19.899 \pm 0.032$ & $r^{\prime}$ & $(30)$ \\
\hline 4794.333 & $20.310 \pm 0.135$ & $r^{\prime}$ & $(30)$ \\
\hline 4795.467 & $20.130 \pm 0.036$ & $r^{\prime}$ & (30) \\
\hline 4796.570 & $>20.119$ & $r^{\prime}$ & $(30)$ \\
\hline 4799.373 & $20.689 \pm 0.148$ & $r^{\prime}$ & (30) \\
\hline 4800.403 & $20.200 \pm 0.225$ & $r^{\prime}$ & (30) \\
\hline 4800.459 & $20.152 \pm 0.138$ & $r^{\prime}$ & (30) \\
\hline 4802.385 & $21.052 \pm 0.140$ & $r^{\prime}$ & (30) \\
\hline 4803.398 & $>19.727$ & $r^{\prime}$ & $(30)$ \\
\hline 4805.328 & $21.155 \pm 0.111$ & $r^{\prime}$ & (30) \\
\hline 4805.384 & $21.470 \pm 0.295$ & $r^{\prime}$ & $(30)$ \\
\hline 4778.393 & $18.367 \pm 0.034$ & $i^{\prime}$ & (30) \\
\hline 4779.465 & $18.884 \pm 0.020$ & $i^{\prime}$ & (30) \\
\hline 4779.906 & $18.909 \pm 0.082$ & $i^{\prime}$ & (31) \\
\hline 4780.478 & $19.020 \pm 0.043$ & $i^{\prime}$ & $(30)$ \\
\hline 4780.922 & $19.552 \pm 0.179$ & $i^{\prime}$ & (31) \\
\hline 4782.411 & $19.591 \pm 0.028$ & $i^{\prime}$ & (30) \\
\hline 4783.489 & $>19.309$ & $i^{\prime}$ & $(30)$ \\
\hline 4785.521 & $20.178 \pm 0.048$ & $i^{\prime}$ & (30) \\
\hline 4786.483 & $>20.126$ & $i^{\prime}$ & $(30)$ \\
\hline 4787.396 & $20.234 \pm 0.099$ & $i^{\prime}$ & (30) \\
\hline 4788.421 & $20.511 \pm 0.044$ & $i^{\prime}$ & (30) \\
\hline 4789.497 & $21.077 \pm 0.184$ & $i^{\prime}$ & (30) \\
\hline 4790.415 & $20.583 \pm 0.142$ & $i^{\prime}$ & (30) \\
\hline 4794.337 & $20.649 \pm 0.186$ & $i^{\prime}$ & (30) \\
\hline 4795.471 & $20.957 \pm 0.059$ & $i^{\prime}$ & $(30)$ \\
\hline 4796.574 & $>19.433$ & $i^{\prime}$ & (30) \\
\hline 4799.376 & $21.295 \pm 0.233$ & $i^{\prime}$ & $(30)$ \\
\hline 4800.464 & $20.993 \pm 0.242$ & $i^{\prime}$ & (30) \\
\hline 4802.389 & $21.300 \pm 0.066$ & $i^{\prime}$ & $(30)$ \\
\hline 4803.401 & $>20.175$ & $i^{\prime}$ & (30) \\
\hline 4805.331 & $>21.000$ & $i^{\prime}$ & (30) \\
\hline 4805.388 & $>21.564$ & $i^{\prime}$ & (30) \\
\hline 4778.396 & $17.866 \pm 0.018$ & $z^{\prime}$ & $(30)$ \\
\hline 4779.468 & $18.131 \pm 0.076$ & $z^{\prime}$ & (30) \\
\hline 4780.482 & $18.500 \pm 0.040$ & $z^{\prime}$ & (30) \\
\hline 4781.465 & $>18.716$ & $z^{\prime}$ & $(30)$ \\
\hline 4782.414 & $19.112 \pm 0.107$ & $z^{\prime}$ & (30) \\
\hline 4783.492 & $19.264 \pm 0.107$ & $z^{\prime}$ & (30) \\
\hline
\end{tabular}


Table 3-Continued

\begin{tabular}{|c|c|c|c|}
\hline $\begin{array}{c}\text { JD } \\
(2,450,000+)\end{array}$ & Mag & Filter & Notes $^{\mathrm{a}}$ \\
\hline 4785.525 & $19.649 \pm 0.066$ & $z^{\prime}$ & $(30)$ \\
\hline 4786.486 & $>19.875$ & $z^{\prime}$ & (30) \\
\hline 4787.400 & $>20.602$ & $z^{\prime}$ & $(30)$ \\
\hline 4788.425 & $20.158 \pm 0.083$ & $z^{\prime}$ & (30) \\
\hline 4789.499 & $20.621 \pm 0.271$ & $z^{\prime}$ & (30) \\
\hline 4790.419 & $>20.364$ & $z^{\prime}$ & (30) \\
\hline 4794.340 & $>20.821$ & $z^{\prime}$ & (30) \\
\hline 4795.474 & $>21.175$ & $z^{\prime}$ & (30) \\
\hline \multirow[t]{2}{*}{4796.578} & $>17.999$ & $z^{\prime}$ & (30) \\
\hline & M31N 2008-12b & & \\
\hline 4842.404 & $17.671 \pm 0.041$ & $B$ & (30) \\
\hline 4846.474 & $17.826 \pm 0.015$ & $B$ & (30) \\
\hline 4851.347 & $17.874 \pm 0.043$ & $B$ & (30) \\
\hline 4856.441 & $18.765 \pm 0.081$ & $B$ & (30) \\
\hline 4859.377 & $19.436 \pm 0.149$ & $B$ & (30) \\
\hline 4842.408 & $17.533 \pm 0.035$ & $V$ & $(30)$ \\
\hline 4846.478 & $17.705 \pm 0.045$ & $V$ & $(30)$ \\
\hline 4851.351 & $17.941 \pm 0.046$ & $V$ & (30) \\
\hline 4856.444 & $18.822 \pm 0.112$ & $V$ & (30) \\
\hline 4859.381 & $19.468 \pm 0.129$ & $V$ & (30) \\
\hline 4829.183 & $>19.7$ & $R$ & (1) \\
\hline 4840.253 & $17.0 \pm 0.1$ & $R$ & (4) \\
\hline 4848.217 & $17.4 \pm 0.15$ & $R$ & (11) \\
\hline 4851.394 & $17.3 \pm 0.15$ & $R$ & (1) \\
\hline 4842.394 & $17.606 \pm 0.030$ & $r^{\prime}$ & $(30)$ \\
\hline 4846.464 & $17.669 \pm 0.036$ & $r^{\prime}$ & (30) \\
\hline 4851.337 & $17.893 \pm 0.033$ & $r^{\prime}$ & (30) \\
\hline 4856.430 & $18.843 \pm 0.058$ & $r^{\prime}$ & (30) \\
\hline 4859.366 & $19.262 \pm 0.105$ & $r^{\prime}$ & $(30)$ \\
\hline 4842.397 & $17.499 \pm 0.049$ & $i^{\prime}$ & $(30)$ \\
\hline 4846.467 & $17.693 \pm 0.032$ & $i^{\prime}$ & (30) \\
\hline 4851.340 & $17.896 \pm 0.044$ & $i^{\prime}$ & (30) \\
\hline 4856.433 & $18.453 \pm 0.080$ & $i^{\prime}$ & (30) \\
\hline 4859.370 & $18.993 \pm 0.089$ & $i^{\prime}$ & (30) \\
\hline 4842.400 & $16.750 \pm 0.022$ & $z^{\prime}$ & $(30)$ \\
\hline 4846.471 & $16.921 \pm 0.043$ & $z^{\prime}$ & $(30)$ \\
\hline 4851.344 & $17.044 \pm 0.049$ & $z^{\prime}$ & (30) \\
\hline 4856.437 & $17.371 \pm 0.070$ & $z^{\prime}$ & (30) \\
\hline
\end{tabular}


Table 3-Continued

\begin{tabular}{|c|c|c|c|}
\hline $\begin{array}{c}\text { JD } \\
(2,450,000+)\end{array}$ & Mag & Filter & Notes $^{\mathrm{a}}$ \\
\hline 4859.374 & $17.533 \pm 0.084$ & $z^{\prime}$ & $(30)$ \\
\hline \multicolumn{4}{|c|}{ M31N 2009-01a } \\
\hline 4872.241 & $>19.3$ & $R$ & (11) \\
\hline \multicolumn{4}{|c|}{ M31N 2009-02a } \\
\hline 4872.254 & $16.91 \pm 0.1$ & $R$ & (11) \\
\hline \multicolumn{4}{|c|}{ M31N 2009-08a } \\
\hline 5053.724 & $17.944 \pm 0.062$ & $B$ & $(30)$ \\
\hline 5057.516 & $18.114 \pm 0.021$ & $B$ & $(30)$ \\
\hline 5058.498 & $18.312 \pm 0.023$ & $B$ & $(30)$ \\
\hline 5059.719 & $18.413 \pm 0.027$ & $B$ & (30) \\
\hline 5060.533 & $18.167 \pm 0.065$ & $B$ & $(30)$ \\
\hline 5061.484 & $18.076 \pm 0.022$ & $B$ & $(30)$ \\
\hline 5061.562 & $18.086 \pm 0.047$ & $B$ & (30) \\
\hline 5062.507 & $18.121 \pm 0.070$ & $B$ & $(30)$ \\
\hline 5063.485 & $18.404 \pm 0.030$ & $B$ & $(30)$ \\
\hline 5064.630 & $17.917 \pm 0.020$ & $B$ & $(30)$ \\
\hline 5065.739 & $17.791 \pm 0.019$ & $B$ & $(30)$ \\
\hline 5066.525 & $17.800 \pm 0.065$ & $B$ & (30) \\
\hline 5067.474 & $17.980 \pm 0.072$ & $B$ & (30) \\
\hline 5069.678 & $18.655 \pm 0.031$ & $B$ & $(30)$ \\
\hline 5070.586 & $18.978 \pm 0.029$ & $B$ & $(30)$ \\
\hline 5071.746 & $>18.911$ & $B$ & $(30)$ \\
\hline 5072.526 & $18.655 \pm 0.059$ & $B$ & $(30)$ \\
\hline 5073.467 & $18.620 \pm 0.092$ & $B$ & $(30)$ \\
\hline 5075.501 & $18.263 \pm 0.098$ & $B$ & $(30)$ \\
\hline 5076.459 & $18.030 \pm 0.068$ & $B$ & (30) \\
\hline 5077.718 & $18.077 \pm 0.021$ & $B$ & (30) \\
\hline 5077.487 & $18.009 \pm 0.021$ & $B$ & $(30)$ \\
\hline 5078.572 & $17.903 \pm 0.021$ & $B$ & $(30)$ \\
\hline 5079.469 & $17.710 \pm 0.021$ & $B$ & (30) \\
\hline 5114.306 & $19.91 \pm 0.10$ & $B$ & (17) \\
\hline 5053.729 & $17.688 \pm 0.060$ & $V$ & $(30)$ \\
\hline 5057.520 & $18.212 \pm 0.022$ & $V$ & $(30)$ \\
\hline 5058.501 & $18.453 \pm 0.025$ & $V$ & (30) \\
\hline 5059.723 & $18.520 \pm 0.092$ & $V$ & (30) \\
\hline 5060.536 & $18.463 \pm 0.071$ & $V$ & (30) \\
\hline 5061.488 & $18.169 \pm 0.063$ & $V$ & $(30)$ \\
\hline 5061.566 & $18.231 \pm 0.050$ & $V$ & $(30)$ \\
\hline
\end{tabular}


Table 3-Continued

\begin{tabular}{|c|c|c|c|}
\hline $\begin{array}{c}\text { JD } \\
(2,450,000+)\end{array}$ & Mag & Filter & Notes $^{\mathrm{a}}$ \\
\hline 5062.511 & $18.321 \pm 0.079$ & $V$ & (30) \\
\hline 5063.489 & $18.686 \pm 0.037$ & $V$ & $(30)$ \\
\hline 5064.633 & $18.167 \pm 0.021$ & $V$ & (30) \\
\hline 5065.742 & $17.858 \pm 0.019$ & $V$ & (30) \\
\hline 5066.528 & $17.885 \pm 0.052$ & $V$ & (30) \\
\hline 5067.478 & $18.320 \pm 0.080$ & $V$ & (30) \\
\hline 5069.681 & $18.949 \pm 0.046$ & $V$ & (30) \\
\hline 5070.590 & $19.263 \pm 0.041$ & $V$ & (30) \\
\hline 5071.749 & $>19.142$ & $V$ & $(30)$ \\
\hline 5072.529 & $18.940 \pm 0.033$ & $V$ & (30) \\
\hline 5073.470 & $18.745 \pm 0.045$ & $V$ & (30) \\
\hline 5074.483 & $18.560 \pm 0.047$ & $V$ & (30) \\
\hline 5075.504 & $18.338 \pm 0.124$ & $V$ & (30) \\
\hline 5076.463 & $18.195 \pm 0.026$ & $V$ & (30) \\
\hline 5077.721 & $18.233 \pm 0.023$ & $V$ & (30) \\
\hline 5077.490 & $18.134 \pm 0.022$ & $V$ & (30) \\
\hline 5078.575 & $18.115 \pm 0.021$ & $V$ & (30) \\
\hline 5079.472 & $17.852 \pm 0.020$ & $V$ & (30) \\
\hline 5092.525 & $18.39 \pm 0.04$ & $V$ & (17) \\
\hline 5095.981 & $18.98 \pm 0.1$ & $V$ & (13) \\
\hline 5114.308 & $20.1 \pm 0.15$ & $V$ & (17) \\
\hline 4985.974 & $>20.2$ & $R$ & (9) \\
\hline 4988.527 & $>19.9$ & $R$ & (1) \\
\hline 5055.923 & $18.7 \pm 0.15$ & $R$ & (8) \\
\hline 5063.605 & $18.8 \pm 0.2$ & $R$ & $(22)$ \\
\hline 5073.441 & $18.3 \pm 0.1$ & $R$ & (1) \\
\hline 5074.366 & $18.3 \pm 0.1$ & $R$ & (1) \\
\hline 5075.384 & $18.0 \pm 0.15$ & $R$ & (1) \\
\hline 5076.478 & $17.8 \pm 0.1$ & $R$ & (1) \\
\hline 5080.413 & $17.8 \pm 0.1$ & $R$ & (1) \\
\hline 5081.393 & $18.4 \pm 0.15$ & $R$ & (1) \\
\hline 5083.357 & $18.5 \pm 0.15$ & $R$ & (1) \\
\hline 5084.267 & $18.2 \pm 0.2$ & $R$ & (1) \\
\hline 5094.451 & $18.3 \pm 0.15$ & $R$ & (3) \\
\hline 5095.968 & $18.52 \pm 0.07$ & $R$ & (13) \\
\hline 5114.304 & $19.2 \pm 0.15$ & $R$ & (17) \\
\hline 5114.486 & $19.2 \pm 0.15$ & $R$ & (3) \\
\hline 5124.491 & $19.5 \pm 0.2$ & $R$ & (3) \\
\hline 5135.632 & $19.3 \pm 0.2$ & $R$ & $(12)$ \\
\hline 5140.546 & $19.7 \pm 0.3$ & $R$ & (1) \\
\hline 5141.359 & $19.9 \pm 0.3$ & $R$ & (1) \\
\hline 5148.536 & $19.7 \pm 0.25$ & $R$ & (1) \\
\hline 5162.281 & $19.3 \pm 0.25$ & $R$ & (5) \\
\hline 5168.173 & $19.4 \pm 0.3$ & $R$ & (7) \\
\hline
\end{tabular}


Table 3-Continued

\begin{tabular}{cccc}
\hline \hline JD & & & \\
$(2,450,000+)$ & Mag & Filter & Notes $^{\mathrm{a}}$ \\
\hline 5173.192 & $19.6 \pm 0.3$ & $R$ & $(1)$ \\
5181.226 & $19.7 \pm 0.3$ & $R$ & $(1)$ \\
5184.717 & $19.9 \pm 0.15$ & $R$ & $(19)$ \\
5199.299 & $20.6 \pm 0.2$ & $R$ & $(12)$ \\
5227.345 & $20.9 \pm 0.25$ & $R$ & $(12)$ \\
5228.383 & $20.9 \pm 0.25$ & $R$ & $(12)$ \\
& & & \\
5050.721 & $18.568 \pm 0.120$ & $r^{\prime}$ & $(30)$ \\
5052.551 & $18.194 \pm 0.057$ & $r^{\prime}$ & $(30)$ \\
5052.556 & $18.248 \pm 0.066$ & $r^{\prime}$ & $(30)$ \\
5052.570 & $18.170 \pm 0.047$ & $r^{\prime}$ & $(30)$ \\
5052.585 & $18.119 \pm 0.042$ & $r^{\prime}$ & $(30)$ \\
5052.591 & $18.198 \pm 0.044$ & $r^{\prime}$ & $(30)$ \\
5052.605 & $18.147 \pm 0.042$ & $r^{\prime}$ & $(30)$ \\
5052.620 & $18.202 \pm 0.019$ & $r^{\prime}$ & $(30)$ \\
5052.626 & $18.186 \pm 0.020$ & $r^{\prime}$ & $(30)$ \\
5052.640 & $18.171 \pm 0.041$ & $r^{\prime}$ & $(30)$ \\
5052.652 & $18.184 \pm 0.046$ & $r^{\prime}$ & $(30)$ \\
5052.660 & $18.098 \pm 0.042$ & $r^{\prime}$ & $(30)$ \\
5052.682 & $18.147 \pm 0.043$ & $r^{\prime}$ & $(30)$ \\
5052.711 & $18.152 \pm 0.061$ & $r^{\prime}$ & $(30)$ \\
5052.721 & $18.154 \pm 0.045$ & $r^{\prime}$ & $(30)$ \\
5052.728 & $18.152 \pm 0.044$ & $r^{\prime}$ & $(30)$ \\
5053.716 & $18.069 \pm 0.054$ & $r^{\prime}$ & $(30)$ \\
5053.505 & $18.160 \pm 0.095$ & $r^{\prime}$ & $(30)$ \\
5053.509 & $18.109 \pm 0.084$ & $r^{\prime}$ & $(30)$ \\
5053.514 & $18.037 \pm 0.026$ & $r^{\prime}$ & $(30)$ \\
5053.528 & $18.143 \pm 0.068$ & $r^{\prime}$ & $(30)$ \\
5053.532 & $18.064 \pm 0.058$ & $r^{\prime}$ & $(30)$ \\
5053.537 & $18.081 \pm 0.057$ & $r^{\prime}$ & $(30)$ \\
5053.541 & $18.101 \pm 0.054$ & $r^{\prime}$ & $(30)$ \\
5054.478 & $18.185 \pm 0.028$ & $r^{\prime}$ & $(30)$ \\
5056.718 & $18.337 \pm 0.074$ & $r^{\prime}$ & $(30)$ \\
5057.510 & $18.357 \pm 0.049$ & $r^{\prime}$ & $(30)$ \\
5058.491 & $18.532 \pm 0.054$ & $r^{\prime}$ & $(30)$ \\
5059.713 & $18.621 \pm 0.099$ & $r^{\prime}$ & $(30)$ \\
5060.526 & $18.472 \pm 0.077$ & $r^{\prime}$ & $(30)$ \\
5061.478 & $18.336 \pm 0.073$ & $r^{\prime}$ & $(30)$ \\
5061.555 & $18.383 \pm 0.045$ & $r^{\prime}$ & $(30)$ \\
5062.501 & $18.278 \pm 0.079$ & $r^{\prime}$ & $(30)$ \\
5063.479 & $18.661 \pm 0.105$ & $r^{\prime}$ & $(30)$ \\
5064.623 & $18.307 \pm 0.047$ & $r^{\prime}$ & $(30)$ \\
5065.733 & $18.073 \pm 0.034$ & $r^{\prime}$ & $(30)$ \\
& $18.055 \pm 0.020$ & $r^{\prime}$ & $(30)$ \\
& $18.349 \pm 0.070$ & $r^{\prime}$ & $(30)$
\end{tabular}


Table 3-Continued

\begin{tabular}{|c|c|c|c|}
\hline $\begin{array}{c}\text { JD } \\
(2,450,000+)\end{array}$ & Mag & Filter & Notes $^{\mathrm{a}}$ \\
\hline 5069.672 & $18.797 \pm 0.041$ & $r^{\prime}$ & $(30)$ \\
\hline 5070.580 & $19.090 \pm 0.087$ & $r^{\prime}$ & (30) \\
\hline 5072.519 & $18.984 \pm 0.076$ & $r^{\prime}$ & (30) \\
\hline 5073.460 & $18.620 \pm 0.065$ & $r^{\prime}$ & $(30)$ \\
\hline 5074.733 & $18.978 \pm 0.017$ & $r^{\prime}$ & $(30)$ \\
\hline 5075.495 & $18.500 \pm 0.044$ & $r^{\prime}$ & (30) \\
\hline 5077.712 & $18.407 \pm 0.049$ & $r^{\prime}$ & (30) \\
\hline 5078.566 & $18.251 \pm 0.018$ & $r^{\prime}$ & (30) \\
\hline 5078.515 & $18.235 \pm 0.019$ & $r^{\prime}$ & $(30)$ \\
\hline 5079.463 & $18.012 \pm 0.016$ & $r^{\prime}$ & (30) \\
\hline 5210.587 & $20.7 \pm 0.2$ & $r^{\prime}$ & (16) \\
\hline 5211.600 & $20.8 \pm 0.25$ & $r^{\prime}$ & (16) \\
\hline \multicolumn{4}{|c|}{ M31N 2009-08b } \\
\hline 5061.546 & $18.559 \pm 0.025$ & $B$ & (30) \\
\hline 5063.625 & $18.782 \pm 0.029$ & $B$ & (30) \\
\hline 5064.662 & $18.960 \pm 0.028$ & $B$ & (30) \\
\hline 5066.594 & $19.165 \pm 0.029$ & $B$ & $(30)$ \\
\hline 5067.459 & $19.298 \pm 0.035$ & $B$ & $(30)$ \\
\hline 5069.600 & $19.592 \pm 0.034$ & $B$ & $(30)$ \\
\hline 5070.567 & $19.847 \pm 0.036$ & $B$ & $(30)$ \\
\hline 5071.522 & $19.695 \pm 0.040$ & $B$ & $(30)$ \\
\hline 5072.496 & $19.899 \pm 0.042$ & $B$ & (30) \\
\hline 5073.554 & $19.944 \pm 0.054$ & $B$ & $(30)$ \\
\hline 5074.717 & $19.950 \pm 0.042$ & $B$ & (30) \\
\hline 5075.517 & $19.971 \pm 0.075$ & $B$ & (30) \\
\hline 5076.485 & $19.813 \pm 0.090$ & $B$ & (30) \\
\hline 5077.471 & $20.165 \pm 0.081$ & $B$ & $(30)$ \\
\hline 5078.492 & $20.380 \pm 0.103$ & $B$ & $(30)$ \\
\hline 5079.523 & $20.383 \pm 0.108$ & $B$ & $(30)$ \\
\hline 5061.549 & $18.424 \pm 0.019$ & $V$ & $(30)$ \\
\hline 5063.628 & $18.665 \pm 0.022$ & $V$ & (30) \\
\hline 5064.665 & $18.776 \pm 0.022$ & $V$ & (30) \\
\hline 5066.596 & $18.991 \pm 0.024$ & $V$ & (30) \\
\hline 5067.462 & $19.056 \pm 0.026$ & $V$ & $(30)$ \\
\hline 5069.603 & $19.420 \pm 0.030$ & $V$ & $(30)$ \\
\hline 5070.570 & $19.559 \pm 0.029$ & $V$ & $(30)$ \\
\hline 5071.523 & $19.465 \pm 0.032$ & $V$ & $(30)$ \\
\hline 5072.499 & $19.709 \pm 0.033$ & $V$ & (30) \\
\hline 5073.556 & $19.652 \pm 0.066$ & $V$ & (30) \\
\hline 5074.719 & $19.792 \pm 0.038$ & $V$ & (30) \\
\hline 5075.520 & $20.053 \pm 0.078$ & $V$ & (30) \\
\hline 5076.487 & $20.117 \pm 0.162$ & $V$ & (30) \\
\hline
\end{tabular}


Table 3-Continued

\begin{tabular}{cccc}
\hline \hline JD & & & \\
$(2,450,000+)$ & Mag & Filter & Notes $^{\mathrm{a}}$ \\
\hline 5077.474 & $19.894 \pm 0.053$ & $V$ & $(30)$ \\
5078.494 & $20.126 \pm 0.075$ & $V$ & $(30)$ \\
5079.526 & $20.298 \pm 0.120$ & $V$ & $(30)$ \\
& & & \\
5061.541 & $17.984 \pm 0.013$ & $r^{\prime}$ & $(30)$ \\
5063.620 & $18.159 \pm 0.016$ & $r^{\prime}$ & $(30)$ \\
5064.657 & $18.269 \pm 0.015$ & $r^{\prime}$ & $(30)$ \\
5066.588 & $18.409 \pm 0.016$ & $r^{\prime}$ & $(30)$ \\
5067.454 & $18.499 \pm 0.019$ & $r^{\prime}$ & $(30)$ \\
5069.595 & $18.723 \pm 0.019$ & $r^{\prime}$ & $(30)$ \\
5070.562 & $18.847 \pm 0.018$ & $r^{\prime}$ & $(30)$ \\
5071.516 & $18.869 \pm 0.019$ & $r^{\prime}$ & $(30)$ \\
5072.491 & $18.970 \pm 0.020$ & $r^{\prime}$ & $(30)$ \\
5073.548 & $18.914 \pm 0.123$ & $r^{\prime}$ & $(30)$ \\
5074.711 & $19.081 \pm 0.021$ & $r^{\prime}$ & $(30)$ \\
5075.512 & $19.089 \pm 0.034$ & $r^{\prime}$ & $(30)$ \\
5076.479 & $19.241 \pm 0.043$ & $r^{\prime}$ & $(30)$ \\
5077.466 & $19.308 \pm 0.033$ & $r^{\prime}$ & $(30)$ \\
5078.487 & $19.338 \pm 0.034$ & $r^{\prime}$ & $(30)$ \\
5079.518 & $19.366 \pm 0.039$ & $r^{\prime}$ & $(30)$ \\
& & & \\
5061.544 & $18.028 \pm 0.015$ & $i^{\prime}$ & $(30)$ \\
5063.622 & $18.262 \pm 0.018$ & $i^{\prime}$ & $(30)$ \\
5064.659 & $18.359 \pm 0.017$ & $i^{\prime}$ & $(30)$ \\
5066.591 & $18.555 \pm 0.019$ & $i^{\prime}$ & $(30)$ \\
5067.456 & $18.641 \pm 0.025$ & $i^{\prime}$ & $(30)$ \\
5069.598 & $18.896 \pm 0.022$ & $i^{\prime}$ & $(30)$ \\
5070.564 & $18.945 \pm 0.023$ & $i^{\prime}$ & $(30)$ \\
5071.517 & $18.798 \pm 0.025$ & $i^{\prime}$ & $(30)$ \\
5072.494 & $19.053 \pm 0.024$ & $i^{\prime}$ & $(30)$ \\
5073.551 & $19.486 \pm 0.091$ & $i^{\prime}$ & $(30)$ \\
5074.714 & $19.083 \pm 0.027$ & $i^{\prime}$ & $(30)$ \\
5075.514 & $19.152 \pm 0.048$ & $i^{\prime}$ & $(30)$ \\
5076.482 & $19.230 \pm 0.065$ & $i^{\prime}$ & $(30)$ \\
5077.469 & $19.314 \pm 0.034$ & $i^{\prime}$ & $(30)$ \\
5078.489 & $19.317 \pm 0.039$ & $i^{\prime}$ & $(30)$ \\
5079.521 & $19.344 \pm 0.052$ & $i^{\prime}$ & $(30)$ \\
\hline 5065.64 .649 & & & \\
\hline 5066.555 & $17.368 \pm 0.434$ & $B$ & $(30)$
\end{tabular}


Table 3-Continued

\begin{tabular}{|c|c|c|c|}
\hline $\begin{array}{c}\text { JD } \\
(2,450,000+)\end{array}$ & Mag & Filter & Notes $^{\mathrm{a}}$ \\
\hline 5067.499 & $18.391 \pm 0.350$ & $B$ & (30) \\
\hline 5069.658 & $18.218 \pm 0.344$ & $B$ & $(30)$ \\
\hline 5070.656 & $18.232 \pm 0.259$ & $B$ & (30) \\
\hline 5071.730 & $>15.710$ & $B$ & (30) \\
\hline 5072.510 & $18.617 \pm 0.414$ & $B$ & (30) \\
\hline 5073.509 & $18.553 \pm 0.317$ & $B$ & (30) \\
\hline 5075.531 & $18.820 \pm 0.443$ & $B$ & (30) \\
\hline 5076.514 & $18.659 \pm 0.368$ & $B$ & (30) \\
\hline 5077.512 & $18.637 \pm 0.369$ & $B$ & $(30)$ \\
\hline 5078.478 & $18.731 \pm 0.428$ & $B$ & (30) \\
\hline 5079.494 & $18.580 \pm 0.312$ & $B$ & (30) \\
\hline 5061.641 & $17.808 \pm 0.359$ & $V$ & $(30)$ \\
\hline 5063.653 & $17.888 \pm 0.327$ & $V$ & (30) \\
\hline 5064.651 & $17.982 \pm 0.284$ & $V$ & (30) \\
\hline 5065.727 & $18.182 \pm 0.381$ & $V$ & (30) \\
\hline 5066.558 & $18.390 \pm 0.650$ & $V$ & (30) \\
\hline 5067.502 & $>17.915$ & $V$ & (30) \\
\hline 5069.661 & $>17.288$ & $V$ & (30) \\
\hline 5070.659 & $>17.900$ & $V$ & $(30)$ \\
\hline 5072.513 & $18.453 \pm 0.522$ & $V$ & $(30)$ \\
\hline 5073.512 & $>18.705$ & $V$ & (30) \\
\hline 5079.497 & $19.155 \pm 0.804$ & $V$ & (30) \\
\hline 5095.981 & $19.9 \pm 0.2$ & $V$ & (13) \\
\hline 4985.974 & $>20.2$ & $R$ & (9) \\
\hline 4988.527 & $>19.9$ & $R$ & (1) \\
\hline 5055.923 & $17.2 \pm 0.1$ & $R$ & (8) \\
\hline 5063.605 & $18.2 \pm 0.35$ & $R$ & $(22)$ \\
\hline 5076.478 & $>20.0$ & $R$ & $(1)$ \\
\hline 5095.968 & $19.46 \pm 0.15$ & $R$ & (13) \\
\hline 5061.633 & $17.685 \pm 0.323$ & $r^{\prime}$ & $(30)$ \\
\hline 5063.644 & $18.122 \pm 0.538$ & $r^{\prime}$ & (30) \\
\hline 5064.643 & $17.888 \pm 0.346$ & $r^{\prime}$ & (30) \\
\hline 5065.719 & $18.070 \pm 0.430$ & $r^{\prime}$ & (30) \\
\hline 5066.550 & $18.066 \pm 0.584$ & $r^{\prime}$ & (30) \\
\hline 5067.494 & $>17.835$ & $r^{\prime}$ & (30) \\
\hline 5069.653 & $>17.945$ & $r^{\prime}$ & (30) \\
\hline 5070.651 & $>17.724$ & $r^{\prime}$ & (30) \\
\hline 5072.505 & $18.329 \pm 0.493$ & $r^{\prime}$ & (30) \\
\hline 5073.504 & $18.646 \pm 0.717$ & $r^{\prime}$ & (30) \\
\hline 5075.526 & $>18.178$ & $r^{\prime}$ & (30) \\
\hline 5077.507 & $>18.368$ & $r^{\prime}$ & (30) \\
\hline 5078.472 & $>18.244$ & $r^{\prime}$ & (30) \\
\hline
\end{tabular}


Table 3-Continued

\begin{tabular}{|c|c|c|c|}
\hline $\begin{array}{c}\text { JD } \\
(2,450,000+)\end{array}$ & Mag & Filter & Notes $^{\mathrm{a}}$ \\
\hline \multirow[t]{2}{*}{5079.489} & $18.848 \pm 0.755$ & $r^{\prime}$ & (30) \\
\hline & \multicolumn{3}{|l|}{ M31N 2009-08e } \\
\hline 5114.306 & $19.56 \pm 0.09$ & $B$ & $(17)$ \\
\hline 5129.835 & $20.12 \pm 0.1$ & $B$ & $(15)$ \\
\hline 5092.543 & $19.09 \pm 0.08$ & $V$ & $(17)$ \\
\hline 5095.981 & $19.32 \pm 0.06$ & $V$ & $(13)$ \\
\hline 5114.308 & $19.7 \pm 0.12$ & $V$ & $(17)$ \\
\hline 5129.824 & $20.15 \pm 0.1$ & $V$ & $(15)$ \\
\hline 5055.923 & $>20.3$ & $R$ & $(8)$ \\
\hline 5063.605 & $>20.1$ & $R$ & $(22)$ \\
\hline 5073.441 & $17.9 \pm 0.1$ & $R$ & $(1)$ \\
\hline 5074.366 & $18.0 \pm 0.1$ & $R$ & (1) \\
\hline 5075.384 & $18.1 \pm 0.15$ & $R$ & (1) \\
\hline 5076.478 & $17.9 \pm 0.1$ & $R$ & (1) \\
\hline 5080.413 & $18.1 \pm 0.1$ & $R$ & (1) \\
\hline 5081.393 & $18.7 \pm 0.15$ & $R$ & (1) \\
\hline 5083.357 & $18.4 \pm 0.2$ & $R$ & (1) \\
\hline 5084.267 & $18.2 \pm 0.2$ & $R$ & (1) \\
\hline 5094.451 & $18.2 \pm 0.15$ & $R$ & (3) \\
\hline 5095.968 & $18.39 \pm 0.04$ & $R$ & (13) \\
\hline 5114.304 & $18.9 \pm 0.11$ & $R$ & (17) \\
\hline 5114.486 & $18.6 \pm 0.15$ & $R$ & $(3)$ \\
\hline 5124.491 & $18.4 \pm 0.15$ & $R$ & (3) \\
\hline 5129.829 & $19.11 \pm 0.08$ & $R$ & (15) \\
\hline 5135.632 & $19.1 \pm 0.15$ & $R$ & (12) \\
\hline 5140.546 & $18.6 \pm 0.25$ & $R$ & (1) \\
\hline 5141.359 & $19.0 \pm 0.25$ & $R$ & (1) \\
\hline 5148.536 & $18.9 \pm 0.2$ & $R$ & (1) \\
\hline 5157.316 & $18.8 \pm 0.3$ & $R$ & (24) \\
\hline 5162.281 & $19.3 \pm 0.25$ & $R$ & $(5)$ \\
\hline 5173.177 & $19.7 \pm 0.3$ & $R$ & (1) \\
\hline 5181.226 & $>19.8$ & $R$ & (1) \\
\hline 5184.717 & $19.9 \pm 0.2$ & $R$ & (19) \\
\hline 5199.299 & $20.0 \pm 0.15$ & $R$ & (12) \\
\hline 5227.345 & $20.7 \pm 0.25$ & $R$ & (12) \\
\hline \multirow[t]{2}{*}{5228.383} & $20.6 \pm 0.25$ & $R$ & (12) \\
\hline & \multicolumn{3}{|l|}{ M31N 2009-09a } \\
\hline 5092.506 & $19.02 \pm 0.05$ & $V$ & (17) \\
\hline
\end{tabular}


Table 3-Continued

\begin{tabular}{|c|c|c|c|}
\hline $\begin{array}{c}\text { JD } \\
(2,450,000+)\end{array}$ & Mag & Filter & Notes $^{\mathrm{a}}$ \\
\hline 5080.413 & $17.5 \pm 0.15$ & $R$ & (1) \\
\hline 5081.393 & $17.9 \pm 0.1$ & $R$ & (1) \\
\hline 5083.357 & $18.1 \pm 0.15$ & $R$ & (1) \\
\hline 5092.506 & $18.21 \pm 0.03$ & $R$ & (17) \\
\hline 5095.993 & $18.18 \pm 0.04$ & $R$ & (13) \\
\hline 5148.578 & $18.0 \pm 0.3$ & $R$ & (1) \\
\hline 5162.513 & $19.0 \pm 0.25$ & $R$ & (5) \\
\hline 5173.203 & $19.3 \pm 0.2$ & $R$ & (1) \\
\hline 5226.321 & $19.7 \pm 0.15$ & $R$ & (12) \\
\hline 5227.306 & $19.7 \pm 0.15$ & $R$ & (12) \\
\hline \multirow[t]{2}{*}{5228.284} & $19.8 \pm 0.15$ & $R$ & (12) \\
\hline & \multicolumn{3}{|l|}{ M31N 2009-10a } \\
\hline 5114.341 & $18.59 \pm 0.10$ & $B$ & (17) \\
\hline 5117.718 & $18.344 \pm 0.114$ & $B$ & (30) \\
\hline 5117.501 & $18.403 \pm 0.029$ & $B$ & (30) \\
\hline 5118.647 & $18.705 \pm 0.034$ & $B$ & (30) \\
\hline 5119.495 & $18.919 \pm 0.030$ & $B$ & (30) \\
\hline 5120.389 & $19.119 \pm 0.033$ & $B$ & (30) \\
\hline 5127.496 & $19.888 \pm 0.048$ & $B$ & $(30)$ \\
\hline 5128.375 & $19.867 \pm 0.041$ & $B$ & $(30)$ \\
\hline 5129.515 & $20.258 \pm 0.057$ & $B$ & $(30)$ \\
\hline 5130.468 & $20.522 \pm 0.054$ & $B$ & (30) \\
\hline 5131.408 & $20.997 \pm 0.137$ & $B$ & (30) \\
\hline 5132.359 & $>21.495$ & $B$ & (30) \\
\hline 5114.361 & $18.33 \pm 0.09$ & $V$ & (17) \\
\hline 5117.721 & $18.574 \pm 0.094$ & $V$ & (30) \\
\hline 5117.505 & $18.367 \pm 0.020$ & $V$ & (30) \\
\hline 5118.650 & $18.710 \pm 0.024$ & $V$ & $(30)$ \\
\hline 5119.498 & $18.950 \pm 0.023$ & $V$ & (30) \\
\hline 5120.392 & $19.113 \pm 0.025$ & $V$ & $(30)$ \\
\hline 5127.499 & $19.776 \pm 0.032$ & $V$ & $(30)$ \\
\hline 5128.378 & $19.922 \pm 0.035$ & $V$ & (30) \\
\hline 5129.518 & $20.252 \pm 0.041$ & $V$ & (30) \\
\hline 5130.472 & $20.743 \pm 0.169$ & $V$ & $(30)$ \\
\hline 5131.412 & $>19.771$ & $V$ & $(30)$ \\
\hline 5114.340 & $17.81 \pm 0.07$ & $R$ & $(17)$ \\
\hline 5162.549 & $>19.9$ & $R$ & $(5)$ \\
\hline \multicolumn{4}{|c|}{ M31N 2009-10b } \\
\hline 5117.487 & $16.260 \pm 0.049$ & $B$ & $(30)$ \\
\hline
\end{tabular}


Table 3-Continued

\begin{tabular}{llcc}
\hline \hline JD & & & \\
$(2,450,000+)$ & \multicolumn{1}{c}{ Mag } & Filter & Notes $^{\mathrm{a}}$ \\
\hline 5117.711 & $15.759 \pm 0.042$ & $B$ & $(30)$ \\
5118.603 & $15.521 \pm 0.042$ & $B$ & $(30)$ \\
5119.488 & $15.316 \pm 0.042$ & $B$ & $(30)$ \\
5120.370 & $15.242 \pm 0.042$ & $B$ & $(30)$ \\
5124.457 & $16.98 \pm 0.04$ & $B$ & $(18)$ \\
5127.489 & $18.338 \pm 0.066$ & $B$ & $(30)$ \\
5128.368 & $18.481 \pm 0.044$ & $B$ & $(30)$ \\
5129.500 & $18.659 \pm 0.077$ & $B$ & $(30)$ \\
5130.475 & $18.827 \pm 0.044$ & $B$ & $(30)$ \\
5131.393 & $18.892 \pm 0.110$ & $B$ & $(30)$ \\
5132.352 & $19.223 \pm 0.053$ & $B$ & $(30)$ \\
5134.341 & $19.108 \pm 0.053$ & $B$ & $(30)$ \\
5135.354 & $19.444 \pm 0.049$ & $B$ & $(30)$ \\
5136.461 & $19.286 \pm 0.065$ & $B$ & $(30)$ \\
5137.400 & $19.649 \pm 0.065$ & $B$ & $(30)$ \\
5138.449 & $>19.033$ & $B$ & $(30)$ \\
5139.652 & $19.411 \pm 0.157$ & $B$ & $(30)$ \\
5140.529 & $20.002 \pm 0.056$ & $B$ & $(30)$ \\
5142.515 & $19.853 \pm 0.172$ & $B$ & $(30)$ \\
5143.620 & $>19.917$ & $B$ & $(30)$ \\
5146.507 & $>19.702$ & $B$ & $(30)$ \\
& & & \\
5117.714 & $15.662 \pm 0.013$ & $V$ & $(30)$ \\
5117.490 & $16.085 \pm 0.012$ & $V$ & $(30)$ \\
5118.606 & $15.424 \pm 0.012$ & $V$ & $(30)$ \\
5119.491 & $15.141 \pm 0.012$ & $V$ & $(30)$ \\
5120.374 & $15.003 \pm 0.011$ & $V$ & $(30)$ \\
5124.458 & $16.46 \pm 0.03$ & $V$ & $(18)$ \\
5127.493 & $18.004 \pm 0.045$ & $V$ & $(30)$ \\
5128.371 & $18.187 \pm 0.016$ & $V$ & $(30)$ \\
5129.503 & $18.489 \pm 0.058$ & $V$ & $(30)$ \\
5129.824 & $18.60 \pm 0.03$ & $V$ & $(15)$ \\
5130.479 & $18.399 \pm 0.020$ & $V$ & $(30)$ \\
5131.397 & $18.903 \pm 0.110$ & $V$ & $(30)$ \\
5132.355 & $18.998 \pm 0.065$ & $V$ & $(30)$ \\
5134.345 & $19.101 \pm 0.025$ & $V$ & $(30)$ \\
5135.357 & $19.275 \pm 0.027$ & $V$ & $(30)$ \\
5136.465 & $18.963 \pm 0.128$ & $V$ & $(30)$ \\
5137.403 & $18.520 \pm 0.173$ & $V$ & $(30)$ \\
5138.452 & $19.170 \pm 0.286$ & $V$ & $(30)$ \\
5139.656 & $19.283 \pm 0.130$ & $V$ & $(30)$ \\
5140.532 & $19.766 \pm 0.044$ & $V$ & $(30)$ \\
5142.519 & $19.628 \pm 0.162$ & $V$ & $(30)$ \\
5143.623 & $>19.170$ & $V$ & $(30)$ \\
5144.343 & $>18.793$ & $V$ & $(30)$
\end{tabular}


Table 3-Continued

\begin{tabular}{|c|c|c|c|}
\hline $\begin{array}{c}\text { JD } \\
(2,450,000+)\end{array}$ & Mag & Filter & Notes $^{a}$ \\
\hline 4985.974 & $>20.2$ & $R$ & (9) \\
\hline 4988.527 & $>20.0$ & $R$ & (1) \\
\hline 5105.262 & $>19.9$ & $R$ & (3) \\
\hline 5114.486 & $18.9 \pm 0.15$ & $R$ & (3) \\
\hline 5124.460 & $15.76 \pm 0.04$ & $R$ & (18) \\
\hline 5124.491 & $15.78 \pm 0.04$ & $R$ & (26) \\
\hline 5129.829 & $17.40 \pm 0.03$ & $R$ & (15) \\
\hline 5129.835 & $18.89 \pm 0.04$ & $B$ & (15) \\
\hline 5135.257 & $17.6 \pm 0.2$ & $R$ & (3) \\
\hline 5135.632 & $17.92 \pm 0.06$ & $R$ & (12) \\
\hline 5140.546 & $18.4 \pm 0.2$ & $R$ & $(1)$ \\
\hline 5141.359 & $18.1 \pm 0.2$ & $R$ & (1) \\
\hline 5148.536 & $18.3 \pm 0.15$ & $R$ & (1) \\
\hline 5157.316 & $18.6 \pm 0.25$ & $R$ & (24) \\
\hline 5162.281 & $18.8 \pm 0.2$ & $R$ & (5) \\
\hline 5173.177 & $19.1 \pm 0.25$ & $R$ & (1) \\
\hline 5181.226 & $19.1 \pm 0.2$ & $R$ & (1) \\
\hline 5199.299 & $19.73 \pm 0.1$ & $R$ & $(12)$ \\
\hline 5227.345 & $20.0 \pm 0.15$ & $R$ & (12) \\
\hline 5228.383 & $20.0 \pm 0.15$ & $R$ & (12) \\
\hline \multicolumn{4}{|c|}{ M31N 2009-10c } \\
\hline 5114.306 & $18.06 \pm 0.04$ & $B$ & (17) \\
\hline 5117.725 & $17.155 \pm 0.023$ & $B$ & (30) \\
\hline 5117.494 & $17.048 \pm 0.017$ & $B$ & (30) \\
\hline 5118.654 & $17.283 \pm 0.024$ & $B$ & (30) \\
\hline 5119.471 & $16.771 \pm 0.019$ & $B$ & (30) \\
\hline 5120.382 & $17.155 \pm 0.017$ & $B$ & (30) \\
\hline 5127.511 & $16.427 \pm 0.011$ & $B$ & (30) \\
\hline 5128.361 & $16.632 \pm 0.037$ & $B$ & (30) \\
\hline 5129.507 & $15.977 \pm 0.030$ & $B$ & (30) \\
\hline 5130.461 & $16.311 \pm 0.028$ & $B$ & (30) \\
\hline 5131.401 & $16.609 \pm 0.013$ & $B$ & (30) \\
\hline 5132.366 & $16.116 \pm 0.013$ & $B$ & (30) \\
\hline 5134.356 & $17.118 \pm 0.016$ & $B$ & (30) \\
\hline 5135.340 & $16.604 \pm 0.011$ & $B$ & (30) \\
\hline 5136.476 & $16.958 \pm 0.016$ & $B$ & (30) \\
\hline 5137.340 & $16.935 \pm 0.061$ & $B$ & (30) \\
\hline 5138.469 & $17.301 \pm 0.037$ & $B$ & (30) \\
\hline 5139.574 & $17.429 \pm 0.020$ & $B$ & (30) \\
\hline 5140.627 & $17.629 \pm 0.025$ & $B$ & (30) \\
\hline 5142.540 & $17.662 \pm 0.110$ & $B$ & (30) \\
\hline 5143.613 & $18.082 \pm 0.207$ & $B$ & (30) \\
\hline
\end{tabular}


Table 3-Continued

\begin{tabular}{|c|c|c|c|}
\hline $\begin{array}{c}\text { JD } \\
(2,450,000+)\end{array}$ & Mag & Filter & Notes $^{\mathrm{a}}$ \\
\hline 5144.325 & $>16.980$ & $B$ & (30) \\
\hline 5145.520 & $>17.737$ & $B$ & (30) \\
\hline 5146.619 & $>18.901$ & $B$ & (30) \\
\hline 5147.498 & $18.559 \pm 0.049$ & $B$ & (30) \\
\hline 5114.308 & $17.69 \pm 0.07$ & $V$ & (17) \\
\hline 5117.728 & $17.452 \pm 0.030$ & $V$ & (30) \\
\hline 5117.497 & $17.207 \pm 0.056$ & $V$ & (30) \\
\hline 5118.657 & $17.437 \pm 0.090$ & $V$ & (30) \\
\hline 5119.475 & $17.124 \pm 0.039$ & $V$ & (30) \\
\hline 5120.385 & $17.336 \pm 0.020$ & $V$ & (30) \\
\hline 5127.515 & $16.513 \pm 0.062$ & $V$ & (30) \\
\hline 5128.364 & $17.116 \pm 0.017$ & $V$ & (30) \\
\hline 5129.511 & $16.413 \pm 0.037$ & $V$ & (30) \\
\hline 5130.464 & $16.985 \pm 0.015$ & $V$ & (30) \\
\hline 5131.404 & $17.026 \pm 0.083$ & $V$ & (30) \\
\hline 5132.369 & $16.673 \pm 0.013$ & $V$ & (30) \\
\hline 5134.359 & $17.380 \pm 0.085$ & $V$ & (30) \\
\hline 5135.343 & $16.998 \pm 0.016$ & $V$ & (30) \\
\hline 5136.479 & $17.425 \pm 0.025$ & $V$ & (30) \\
\hline 5137.343 & $17.537 \pm 0.026$ & $V$ & (30) \\
\hline 5138.472 & $>17.347$ & $V$ & (30) \\
\hline 5139.578 & $17.931 \pm 0.038$ & $V$ & (30) \\
\hline 5140.630 & $18.082 \pm 0.042$ & $V$ & (30) \\
\hline 5142.543 & $18.013 \pm 0.148$ & $V$ & (30) \\
\hline 5143.616 & $>17.542$ & $V$ & (30) \\
\hline 5144.328 & $>17.223$ & $V$ & $(30)$ \\
\hline 5114.304 & $17.50 \pm 0.08$ & $R$ & (17) \\
\hline 5114.486 & $17.7 \pm 0.3$ & $R$ & (3) \\
\hline 5124.491 & $16.4 \pm 0.15$ & $R$ & (3) \\
\hline 5135.257 & $16.7 \pm 0.3$ & $R$ & (3) \\
\hline 5135.632 & $17.03 \pm 0.1$ & $R$ & (12) \\
\hline 5140.546 & $17.4 \pm 0.25$ & $R$ & $(1)$ \\
\hline 5141.359 & $17.6 \pm 0.2$ & $R$ & (1) \\
\hline \multicolumn{4}{|c|}{ M31N 2009-11a } \\
\hline 5142.606 & $18.504 \pm 0.078$ & $B$ & $(30)$ \\
\hline 5142.522 & $18.411 \pm 0.073$ & $B$ & (30) \\
\hline 5143.606 & $18.858 \pm 0.132$ & $B$ & (30) \\
\hline 5144.311 & $18.533 \pm 0.180$ & $B$ & (30) \\
\hline 5145.513 & $19.017 \pm 0.038$ & $B$ & (30) \\
\hline 5146.498 & $19.211 \pm 0.211$ & $B$ & (30) \\
\hline 5147.477 & $19.119 \pm 0.057$ & $B$ & (30) \\
\hline
\end{tabular}


Table 3-Continued

\begin{tabular}{|c|c|c|c|}
\hline $\begin{array}{c}\text { JD } \\
(2,450,000+)\end{array}$ & Mag & Filter & Notes $^{\mathrm{a}}$ \\
\hline 5149.358 & $19.325 \pm 0.025$ & $B$ & (30) \\
\hline 5150.410 & $19.610 \pm 0.086$ & $B$ & (30) \\
\hline 5151.559 & $19.356 \pm 0.159$ & $B$ & (30) \\
\hline 5154.592 & $19.705 \pm 0.043$ & $B$ & $(30)$ \\
\hline 5155.334 & $19.532 \pm 0.098$ & $B$ & (30) \\
\hline 5156.309 & $19.550 \pm 0.184$ & $B$ & $(30)$ \\
\hline 5157.572 & $19.840 \pm 0.184$ & $B$ & (30) \\
\hline 5159.438 & $19.810 \pm 0.110$ & $B$ & (30) \\
\hline 5161.539 & $20.012 \pm 0.239$ & $B$ & (30) \\
\hline 5164.398 & $21.234 \pm 0.317$ & $B$ & (30) \\
\hline 5168.433 & $>20.525$ & $B$ & (30) \\
\hline 5169.522 & $>20.686$ & $B$ & $(30)$ \\
\hline 5142.609 & $18.146 \pm 0.049$ & $V$ & (30) \\
\hline 5143.609 & $18.206 \pm 0.088$ & $V$ & $(30)$ \\
\hline 5144.315 & $18.801 \pm 0.165$ & $V$ & (30) \\
\hline 5145.516 & $18.639 \pm 0.216$ & $V$ & (30) \\
\hline 5146.501 & $18.686 \pm 0.169$ & $V$ & (30) \\
\hline 5147.480 & $18.835 \pm 0.050$ & $V$ & (30) \\
\hline 5149.361 & $19.187 \pm 0.023$ & $V$ & (30) \\
\hline 5150.413 & $19.482 \pm 0.025$ & $V$ & $(30)$ \\
\hline 5151.563 & $19.392 \pm 0.119$ & $V$ & $(30)$ \\
\hline 5154.595 & $19.310 \pm 0.141$ & $V$ & $(30)$ \\
\hline 5155.338 & $19.563 \pm 0.030$ & $V$ & (30) \\
\hline 5156.312 & $19.557 \pm 0.130$ & $V$ & (30) \\
\hline 5157.575 & $19.885 \pm 0.055$ & $V$ & (30) \\
\hline 5159.441 & $19.896 \pm 0.086$ & $V$ & (30) \\
\hline 5161.542 & $20.160 \pm 0.063$ & $V$ & (30) \\
\hline 5164.401 & $20.249 \pm 0.059$ & $V$ & (30) \\
\hline 5168.436 & $>19.597$ & $V$ & (30) \\
\hline 5169.525 & $19.910 \pm 0.134$ & $V$ & (30) \\
\hline 5140.463 & $17.6 \pm 0.1$ & $R$ & (1) \\
\hline 5141.221 & $17.74 \pm 0.05$ & $R$ & (5) \\
\hline 5148.564 & $18.4 \pm 0.25$ & $R$ & (1) \\
\hline 5162.534 & $19.4 \pm 0.3$ & $R$ & (5) \\
\hline 5160.803 & $19.71 \pm 0.08$ & $r^{\prime}$ & (10) \\
\hline 5209.656 & $21.5 \pm 0.15$ & $r^{\prime}$ & $(16)$ \\
\hline 5160.806 & $20.35 \pm 0.07$ & $g^{\prime}$ & (10) \\
\hline \multicolumn{4}{|c|}{ M31N 2009-11b (recurrent nova) } \\
\hline 5154.412 & $19.994 \pm 0.119$ & $B$ & $(30)$ \\
\hline 5156.323 & $19.511 \pm 0.120$ & $B$ & (30) \\
\hline
\end{tabular}


Table 3-Continued

\begin{tabular}{|c|c|c|c|}
\hline $\begin{array}{c}\text { JD } \\
(2,450,000+)\end{array}$ & Mag & Filter & Notes $^{\mathrm{a}}$ \\
\hline 5159.406 & $19.859 \pm 0.043$ & $B$ & $(30)$ \\
\hline 5162.503 & $20.347 \pm 0.123$ & $B$ & (30) \\
\hline 5168.480 & $>19.123$ & $B$ & (30) \\
\hline 5169.340 & $>19.175$ & $B$ & (30) \\
\hline 5170.551 & $19.519 \pm 0.098$ & $B$ & (30) \\
\hline 5171.365 & $19.439 \pm 0.037$ & $B$ & $(30)$ \\
\hline 5172.330 & $19.659 \pm 0.036$ & $B$ & (30) \\
\hline 5154.415 & $20.079 \pm 0.139$ & $V$ & $(30)$ \\
\hline 5156.326 & $19.299 \pm 0.033$ & $V$ & (30) \\
\hline 5159.409 & $19.972 \pm 0.040$ & $V$ & (30) \\
\hline 5162.507 & $20.339 \pm 0.055$ & $V$ & (30) \\
\hline 5168.483 & $>18.741$ & $V$ & (30) \\
\hline 5169.343 & $19.372 \pm 0.145$ & $V$ & $(30)$ \\
\hline 5170.554 & $19.482 \pm 0.039$ & $V$ & $(30)$ \\
\hline 5171.368 & $19.490 \pm 0.086$ & $V$ & (30) \\
\hline 5172.333 & $19.564 \pm 0.032$ & $V$ & (30) \\
\hline 5148.536 & $18.9 \pm 0.15$ & $R$ & (1) \\
\hline 5135.632 & $18.6 \pm 0.1$ & $R$ & (12) \\
\hline 5162.281 & $19.3 \pm 0.25$ & $R$ & $(5)$ \\
\hline 5162.513 & $19.6 \pm 0.3$ & $R$ & (5) \\
\hline 5173.177 & $19.3 \pm 0.2$ & $R$ & (1) \\
\hline 5173.203 & $19.2 \pm 0.2$ & $R$ & (1) \\
\hline 5181.226 & $19.7 \pm 0.25$ & $R$ & (1) \\
\hline 5199.299 & $20.05 \pm 0.1$ & $R$ & (12) \\
\hline 5227.306 & $20.0 \pm 0.15$ & $R$ & (12) \\
\hline 5228.284 & $20.0 \pm 0.15$ & $R$ & (12) \\
\hline 5209.616 & $20.9 \pm 0.15$ & $g^{\prime}$ & (16) \\
\hline 5207.591 & $21.2 \pm 0.1$ & $g^{\prime}$ & (16) \\
\hline 5207.599 & $20.66 \pm 0.1$ & $r^{\prime}$ & (16) \\
\hline 5209.621 & $20.6 \pm 0.1$ & $r^{\prime}$ & (16) \\
\hline \multicolumn{4}{|c|}{ M31N 2009-11c } \\
\hline 5145.323 & $19.144 \pm 0.029$ & $B$ & (30) \\
\hline 5146.516 & $18.614 \pm 0.023$ & $B$ & (30) \\
\hline 5147.487 & $18.203 \pm 0.018$ & $B$ & (30) \\
\hline 5148.383 & $17.453 \pm 0.016$ & $B$ & (30) \\
\hline 5149.503 & $18.981 \pm 0.022$ & $B$ & (30) \\
\hline 5150.403 & $18.864 \pm 0.022$ & $B$ & (30) \\
\hline 5154.422 & $18.636 \pm 0.019$ & $B$ & (30) \\
\hline 5156.337 & $18.650 \pm 0.021$ & $B$ & (30) \\
\hline
\end{tabular}


Table 3-Continued

\begin{tabular}{|c|c|c|c|}
\hline $\begin{array}{c}\text { JD } \\
(2,450,000+)\end{array}$ & Mag & Filter & Notes $^{\mathrm{a}}$ \\
\hline 5161.546 & $18.883 \pm 0.027$ & $B$ & $(30)$ \\
\hline 5162.535 & $18.775 \pm 0.026$ & $B$ & $(30)$ \\
\hline 5168.457 & $19.075 \pm 0.256$ & $B$ & (30) \\
\hline 5169.333 & $>18.858$ & $B$ & (30) \\
\hline 5170.584 & $19.676 \pm 0.056$ & $B$ & (30) \\
\hline 5171.373 & $19.393 \pm 0.027$ & $B$ & (30) \\
\hline 5172.363 & $19.375 \pm 0.028$ & $B$ & (30) \\
\hline 5173.509 & $19.635 \pm 0.042$ & $B$ & (30) \\
\hline 5145.326 & $18.409 \pm 0.043$ & $V$ & $(30)$ \\
\hline 5146.519 & $17.879 \pm 0.040$ & $V$ & (30) \\
\hline 5147.490 & $17.497 \pm 0.039$ & $V$ & (30) \\
\hline 5148.386 & $16.770 \pm 0.039$ & $V$ & (30) \\
\hline 5149.506 & $18.122 \pm 0.040$ & $V$ & (30) \\
\hline 5150.406 & $18.178 \pm 0.040$ & $V$ & (30) \\
\hline 5154.425 & $17.959 \pm 0.040$ & $V$ & (30) \\
\hline 5156.340 & $18.172 \pm 0.040$ & $V$ & $(30)$ \\
\hline 5161.549 & $18.710 \pm 0.044$ & $V$ & (30) \\
\hline 5162.538 & $18.292 \pm 0.042$ & $V$ & (30) \\
\hline 5168.461 & $18.196 \pm 0.138$ & $V$ & $(30)$ \\
\hline 5169.336 & $18.742 \pm 0.280$ & $V$ & (30) \\
\hline 5170.587 & $19.065 \pm 0.053$ & $V$ & (30) \\
\hline 5171.375 & $18.870 \pm 0.044$ & $V$ & (30) \\
\hline 5172.366 & $19.070 \pm 0.044$ & $V$ & (30) \\
\hline 5173.512 & $19.392 \pm 0.062$ & $V$ & (30) \\
\hline 5135.632 & $>21.4$ & $R$ & (12) \\
\hline 5140.463 & $>20.3$ & $R$ & (1) \\
\hline 5141.359 & $19.9 \pm 0.3$ & $R$ & (1) \\
\hline 5148.536 & $17.03 \pm 0.1$ & $R$ & (1) \\
\hline 5154.424 & $17.8 \pm 0.15$ & $R$ & (7) \\
\hline 5157.316 & $17.9 \pm 0.1$ & $R$ & (24) \\
\hline 5158.542 & $17.9 \pm 0.1$ & $R$ & (1) \\
\hline 5161.164 & $18.4 \pm 0.15$ & $R$ & (7) \\
\hline 5162.281 & $18.1 \pm 0.15$ & $R$ & (5) \\
\hline 5162.513 & $18.2 \pm 0.15$ & $R$ & (5) \\
\hline 5168.173 & $18.3 \pm 0.1$ & $R$ & (7) \\
\hline 5169.202 & $18.6 \pm 0.2$ & $R$ & (1) \\
\hline 5173.177 & $18.6 \pm 0.15$ & $R$ & (1) \\
\hline 5173.192 & $18.7 \pm 0.15$ & $R$ & (1) \\
\hline 5173.203 & $18.5 \pm 0.15$ & $R$ & (1) \\
\hline 5181.226 & $18.9 \pm 0.15$ & $R$ & (1) \\
\hline 5184.717 & $19.22 \pm 0.1$ & $R$ & (19) \\
\hline 5227.306 & $>22.0$ & $R$ & (12) \\
\hline
\end{tabular}


Table 3-Continued

\begin{tabular}{|c|c|c|c|}
\hline $\begin{array}{c}\text { JD } \\
(2,450,000+)\end{array}$ & Mag & Filter & Notes $^{\mathrm{a}}$ \\
\hline 5156.756 & $18.86 \pm 0.08$ & $g^{\prime}$ & (10) \\
\hline 5156.759 & $18.32 \pm 0.05$ & $r^{\prime}$ & (10) \\
\hline 5209.631 & $>21.1$ & $r^{\prime}$ & (16) \\
\hline \multicolumn{4}{|c|}{ M31N 2009-11d } \\
\hline 5156.330 & $17.278 \pm 0.016$ & $B$ & (30) \\
\hline 5157.579 & $18.659 \pm 0.102$ & $B$ & (30) \\
\hline 5159.445 & $18.123 \pm 0.019$ & $B$ & $(30)$ \\
\hline 5161.532 & $18.554 \pm 0.023$ & $B$ & $(30)$ \\
\hline 5168.464 & $>19.753$ & $B$ & (30) \\
\hline 5169.515 & $19.699 \pm 0.040$ & $B$ & $(30)$ \\
\hline 5170.529 & $20.139 \pm 0.063$ & $B$ & (30) \\
\hline 5171.358 & $20.038 \pm 0.035$ & $B$ & $(30)$ \\
\hline 5172.556 & $19.787 \pm 0.112$ & $B$ & (30) \\
\hline 5173.365 & $19.997 \pm 0.032$ & $B$ & (30) \\
\hline 5176.376 & $>19.722$ & $B$ & $(30)$ \\
\hline 5156.333 & $17.050 \pm 0.012$ & $V$ & $(30)$ \\
\hline 5157.582 & $18.568 \pm 0.101$ & $V$ & $(30)$ \\
\hline 5159.448 & $18.047 \pm 0.014$ & $V$ & $(30)$ \\
\hline 5161.535 & $18.288 \pm 0.075$ & $V$ & $(30)$ \\
\hline 5168.468 & $>19.415$ & $V$ & $(30)$ \\
\hline 5169.518 & $20.034 \pm 0.139$ & $V$ & (30) \\
\hline 5170.532 & $20.696 \pm 0.077$ & $V$ & (30) \\
\hline 5171.361 & $19.912 \pm 0.098$ & $V$ & $(30)$ \\
\hline 5172.559 & $20.501 \pm 0.055$ & $V$ & (30) \\
\hline 5173.368 & $20.375 \pm 0.035$ & $V$ & (30) \\
\hline 5176.378 & $20.523 \pm 0.077$ & $V$ & (30) \\
\hline 5157.299 & $17.26 \pm 0.10$ & $R$ & $(24)$ \\
\hline 5158.560 & $17.3 \pm 0.2$ & $R$ & (1) \\
\hline 5162.525 & $17.6 \pm 0.15$ & $R$ & (5) \\
\hline 5168.505 & $18.1 \pm 0.2$ & $R$ & (7) \\
\hline 5173.192 & $18.7 \pm 0.15$ & $R$ & (1) \\
\hline 5227.379 & $>21.5$ & $R$ & (12) \\
\hline 5156.787 & $16.53 \pm 0.10$ & $g^{\prime}$ & (10) \\
\hline 5207.619 & $21.3 \pm 0.1$ & $g^{\prime}$ & (16) \\
\hline 5156.785 & $16.43 \pm 0.08$ & $r^{\prime}$ & (10) \\
\hline 5207.626 & $21.3 \pm 0.1$ & $r^{\prime}$ & (16) \\
\hline
\end{tabular}

M31N 2009-11e 
Table 3-Continued

\begin{tabular}{cccc}
\hline \hline JD & & & \\
$(2,450,000+)$ & Mag & Filter & Notes $^{\mathrm{a}}$ \\
\hline & & & \\
\hline 5161.575 & $18.122 \pm 0.074$ & $B$ & $(30)$ \\
5162.524 & $17.445 \pm 0.041$ & $B$ & $(30)$ \\
5168.445 & $17.496 \pm 0.018$ & $B$ & $(30)$ \\
5169.326 & $17.279 \pm 0.079$ & $B$ & $(30)$ \\
5170.572 & $17.110 \pm 0.014$ & $B$ & $(30)$ \\
5171.379 & $16.869 \pm 0.011$ & $B$ & $(30)$ \\
5172.355 & $16.982 \pm 0.011$ & $B$ & $(30)$ \\
5173.411 & $18.485 \pm 0.064$ & $B$ & $(30)$ \\
5176.408 & $18.423 \pm 0.033$ & $B$ & $(30)$ \\
& & & \\
5161.578 & $18.254 \pm 0.066$ & $V$ & $(30)$ \\
5162.528 & $17.786 \pm 0.014$ & $V$ & $(30)$ \\
5168.448 & $17.810 \pm 0.061$ & $V$ & $(30)$ \\
5169.329 & $17.590 \pm 0.104$ & $V$ & $(30)$ \\
5170.575 & $17.418 \pm 0.038$ & $V$ & $(30)$ \\
5171.382 & $17.073 \pm 0.010$ & $V$ & $(30)$ \\
5173.415 & $19.005 \pm 0.094$ & $V$ & $(30)$ \\
5176.412 & $19.185 \pm 0.269$ & $V$ & $(30)$ \\
5148.536 & $>20.6$ & & \\
5157.316 & $17.8 \pm 0.15$ & $R$ & $(1)$ \\
5158.542 & $17.0 \pm 0.1$ & $R$ & $(1)$ \\
5161.164 & $17.7 \pm 0.1$ & $R$ & $(7)$ \\
5162.281 & $17.4 \pm 0.1$ & $R$ & $(5)$ \\
5162.513 & $17.5 \pm 0.1$ & $R$ & $(5)$ \\
5168.173 & $17.5 \pm 0.1$ & $R$ & $(7)$ \\
5169.202 & $17.2 \pm 0.2$ & $R$ & $(1)$ \\
5173.177 & $18.1 \pm 0.1$ & $R$ & $(1)$ \\
5173.203 & $18.1 \pm 0.1$ & $R$ & $(1)$ \\
5181.226 & $18.5 \pm 0.15$ & $R$ & $(1)$ \\
5184.717 & $18.96 \pm 0.09$ & $R$ & $(19)$ \\
5186.730 & $18.8 \pm 0.25$ & $R$ & $(20)$ \\
5199.299 & $19.09 \pm 0.08$ & $R$ & $(12)$ \\
5227.345 & $19.92 \pm 0.10$ & $R$ & $(12)$ \\
5228.383 & $19.40 \pm 0.10$ & $R$ & $(12)$ \\
5157.691 & $17.80 \pm 0.09$ & $g^{\prime}$ & $(10)$ \\
5158.715 & $17.29 \pm 0.10$ & $g^{\prime}$ & $(10)$ \\
5207.591 & $19.10 \pm 0.09$ & $g^{\prime}$ & $(16)$ \\
5210.592 & $19.65 \pm 0.10$ & $g^{\prime}$ & $(16)$ \\
5157.688 & $17.80 \pm 0.04$ & & \\
5158.713 & $17.33 \pm 0.03$ & $r^{\prime}$ & $(10)$ \\
5207.599 & $18.91 \pm 0.08$ & $r^{\prime}$ & $(16)$
\end{tabular}


Table 3-Continued

\begin{tabular}{lccc}
\hline \hline $\begin{array}{c}\text { JD } \\
(2,450,000+)\end{array}$ & Mag & Filter & Notes $^{\mathrm{a}}$ \\
\hline 5210.598 & $19.45 \pm 0.09$ & $r^{\prime}$ & $(16)$ \\
\hline
\end{tabular}

a Observers: (1) K. Hornoch, Ondřejov 0.65m; (2) K. Hornoch, Lelekovice 0.35-m; (3) P. Kušnirák, Ondřejov 0.65-m; (4) M. Wolf, Ondřejov 0.65-m; (5) K. Hornoch \& M. Wolf, Ondřejov 0.65$\mathrm{m}$; (6) M. Wolf i\& P. Zasche, Ondřejov 0.65-m; (7) K. Hornoch \& P. Zasche, Ondřejov 0.65-m; (8) P. Zasche, San Pedro Mártir 0.84-m; (9) O. Pejcha, MDM 1.3-m; (10) O. Pejcha, MDM 2.4-m; (11) K. Hornoch \& P. Šedinová, Ondřejov 0.65m; (12) P. Kubánek, J. Gorosabel i\& M. Jelínek, Calar Alto 1.23-m; (13) P. Kubánek, MDM 2.4m; (14) P. Garnavich \& A. Karska, MGIO 1.83-m VATT; (15) P. Garnavich, K. Thorne \& K. Morhig, MGIO 1.83-m VATT; (16) J. Prieto \& R. Khan, MDM 2.4-m; (17) A. Valeev \& O. Sholukhova, SAO 6-m; (18) V. L. Afanasiev \& S. N. Dodonov, SAO 6-m; (19) T. Farnham \& B. Mueller, KPNO 2.1-m; (20) N. Samarasinha \& B. Mueller, KPNO 2.1-m; (21) M. Burleigh \& S. Casewell, La Palma 2.5-m INT; (22) A. Galád, AGO Modra 0.60-m; (23) P. Cagaš, Zlín 0.26-m; (24) K. Hornoch \& P. Kušnirák, Ondřejov 0.65-m; (25) P. Kušnirák \& T. Henych, Ondřejov 0.65-m; (26) P. Kušnirák \& Z. Bardon, Ondřejov 0.65-m; (27) K. Hornoch \& M. Tukinská, Ondřejov 0.65-m; (28) P. Zasche \& Ondřejov, 0.65-m; (29) Darnley et al. 2004; La Palma 2.5-m INT; (30) La Palma 2.0-m LT; (31) Mt. Haleakala 2.0-m FTN; (32) P. Kušnirák, L. Šarounová \& M. Wolf, Ondřejov 0.65-m; (33) P. Garnavich, MGIO 1.83-m VATT; (34) P. Garnavich \& B. Tucker, MGIO 1.83-m VATT; (35) P. Garnavich \& C. Kennedy, MGIO 1.83-m VATT; (36) P. Garnavich \& J. Gallagher, MGIO 1.83-m VATT; (37) P. Garnavich, KPNO 3.5-m WIYN; (38) D. Mackey, La Palma 2.5-m INT. 
Table 4. Full Spectroscopic Sample of M31 Novae

\begin{tabular}{|c|c|c|c|c|c|c|}
\hline Nova & $\begin{array}{c}\Delta \alpha \cos \delta \\
\left({ }^{\prime}\right)\end{array}$ & $\begin{array}{l}\Delta \delta \\
\left({ }^{\prime}\right)\end{array}$ & $\begin{array}{c}a \\
(')\end{array}$ & $\begin{array}{c}\text { Discovery } \\
\text { mag (Filter) }\end{array}$ & Type & References $^{\mathrm{a}}$ \\
\hline M31N 1981-09a & -4.76 & -2.89 & 5.67 & $16.4(\mathrm{H})$ & Fe II & 1 \\
\hline M31N 1981-09b & -0.65 & 2.10 & 2.96 & $14.9(\mathrm{H})$ & $\mathrm{Fe} I I$ & 1 \\
\hline $\mathrm{M} 31 \mathrm{~N} 1981-09 \mathrm{c}$ & 0.78 & 2.87 & 3.43 & $15.0(\mathrm{H})$ & Fe II & 1 \\
\hline M31N 1981-09d & 4.11 & -2.16 & 8.07 & $15.8(\mathrm{H})$ & $\mathrm{Fe} I I$ & 1 \\
\hline M31N 1986-08a & -4.35 & -1.64 & 4.98 & $16.3(\mathrm{H})$ & Fe II & 2 \\
\hline M31N 1987-09a & 7.80 & 2.13 & 10.57 & 19.4(B) & $\mathrm{Fe} I \mathrm{II}$ & 2 \\
\hline M31N 1987-10a & -0.33 & 1.75 & 2.28 & $18.4(\mathrm{~B})$ & Fe II & 2 \\
\hline M31N 1989-08a & 6.47 & -0.29 & 10.36 & $17.9(\mathrm{~B})$ & $\mathrm{He} / \mathrm{N}$ & 2 \\
\hline M31N 1989-08b & -1.01 & 0.00 & 1.10 & $19.3(\mathrm{~B})$ & $\mathrm{Fe}$ II & 2 \\
\hline M31N 1989-08c & -5.22 & -4.41 & 6.84 & $17.9(\mathrm{~B})$ & $\mathrm{Fe} I \mathrm{II}$ & 2 \\
\hline M31N 1989-09a & 2.15 & 4.05 & 5.06 & $15.5(\mathrm{H})$ & $\mathrm{Fe} I \mathrm{II}$ & 2 \\
\hline M31N 1989-10a & 0.36 & 3.10 & 3.91 & $17.6(\mathrm{~B})$ & Fe II & 2 \\
\hline M31N 1990-10b & -1.53 & -4.32 & 5.44 & $17.6(\mathrm{~B})$ & $\mathrm{Fe} I \mathrm{II}$ & 3 \\
\hline M31N 1992-11b & 3.07 & -1.49 & 5.04 & $17.2(\mathrm{~V})$ & Fe II & 3 \\
\hline M31N 1993-06a & 0.91 & 1.31 & 1.64 & $15.8(\mathrm{R})$ & Fe II: & 3 \\
\hline M31N 1993-08a & 0.14 & -1.70 & 1.64 & $15.8(\mathrm{R})$ & $\mathrm{He} / \mathrm{N}$ : & 3 \\
\hline M31N 1993-10g & 0.63 & 1.87 & 2.17 & $16.7(\mathrm{H})$ & Fe II & 3 \\
\hline M31N 1993-11c & 1.08 & 1.32 & 1.73 & $15.8(\mathrm{H})$ & Fe II: & 3 \\
\hline M31N 1998-09d & 0.43 & -1.33 & 1.78 & $16.5(\mathrm{~B})$ & $\mathrm{Fe} I \mathrm{II}$ & 3 \\
\hline M31N 1999-06a & 0.97 & -1.05 & 1.89 & $17.8(\mathrm{R})$ & $\mathrm{Fe} I \mathrm{II}$ & 3 \\
\hline M31N 1999-08f & -0.61 & 3.04 & 4.44 & $17.0(\mathrm{w})$ & Fe II: & 3 \\
\hline M31N 1999-10a & 1.00 & 0.39 & 1.10 & $17.5(\mathrm{w})$ & Fe II: & 3 \\
\hline M31N 2001-10a & 3.56 & -3.96 & 10.81 & $17.0(\mathrm{R})$ & Fe II & 3 \\
\hline M31N 2001-12a & -0.55 & 0.26 & 0.68 & $15.5(\mathrm{R})$ & $\mathrm{Fe} I \mathrm{II}$ & 3 \\
\hline M31N 2002-01b & -1.97 & 2.25 & 4.60 & $16.8(\mathrm{R})$ & $\mathrm{He} / \mathrm{N}:$ & 3 \\
\hline M31N 2002-08a & -2.53 & -9.93 & 14.01 & $17.1(\mathrm{R})$ & Fe II: & 3 \\
\hline M31N 2004-08b & 7.98 & 0.54 & 12.60 & $17.3(\mathrm{R})$ & $\mathrm{Fe} I I$ & 3 \\
\hline M31N 2004-09a & -0.77 & -1.44 & 1.72 & $17.5(\mathrm{R})$ & $\mathrm{Fe} I \mathrm{II}$ & 3 \\
\hline M31N 2004-11a & -0.29 & 2.32 & 3.03 & $16.5(\mathrm{R})$ & $\mathrm{Fe}$ II & 3 \\
\hline M31N 2004-11b & 4.34 & 1.93 & 4.97 & $16.6(\mathrm{R})$ & $\mathrm{He} / \mathrm{N}:$ & 3 \\
\hline M31N 2005-01a & -3.00 & 0.46 & 3.98 & $15.0(\mathrm{R})$ & $\mathrm{Fe} I I$ & 3 \\
\hline M31N 2005-06d & 26.35 & 17.61 & 44.21 & $15.7(\mathrm{w})$ & $\mathrm{He} / \mathrm{N}$ & 4 \\
\hline M31N 2005-07a & 1.21 & 4.52 & 5.85 & $17.4(\mathrm{R})$ & FeII: & 3 \\
\hline M31N 2005-09a & 1.48 & 3.84 & 4.76 & $16.7(\mathrm{R})$ & $\mathrm{Fe} I I$ & 4 \\
\hline M31N 2005-09b & -44.77 & -56.00 & 71.73 & $16.5(\mathrm{w})$ & $\mathrm{Fe} \mathrm{II}^{\mathrm{b}}$ & 4 \\
\hline M31N 2005-09c & -43.70 & -48.34 & 66.76 & $16.0(\mathrm{w})$ & Fe II & 5 \\
\hline M31N 2006-06a & 5.16 & -2.40 & 11.09 & $17.5(\mathrm{R})$ & $\mathrm{Fe} I \mathrm{II}$ & 4 \\
\hline M31N 2006-09c & -0.37 & -7.39 & 11.61 & $16.8(\mathrm{w})$ & $\mathrm{Fe} I I$ & 3 \\
\hline M31N 2006-10a & -11.50 & -4.36 & 17.31 & $18.7(\mathrm{R})$ & $\mathrm{Fe} I \mathrm{II}$ & 3 \\
\hline M31N 2006-10b & -37.25 & -24.81 & 63.14 & $16.4(\mathrm{w})$ & Hy & 3 \\
\hline M31N 2006-11a & 2.35 & -9.84 & 20.46 & $17.3(\mathrm{w})$ & $\mathrm{Fe} I \mathrm{II}$ & 3 \\
\hline M31N 2006-12a & -4.38 & -2.39 & 5.11 & $17.8(\mathrm{R})$ & $\mathrm{Fe} I \mathrm{II}$ & 3 \\
\hline M31N 2006-12b & -6.26 & -8.41 & 10.88 & $18.0(\mathrm{R})$ & $\mathrm{Fe}$ II & 3 \\
\hline M31N 2007-02a & -19.95 & -31.22 & 40.21 & $16.3(\mathrm{w})$ & $\mathrm{Fe} I I$ & 3 \\
\hline M31N 2007-02b & -12.04 & -1.57 & 21.34 & $16.7(\mathrm{R})$ & Fe II: & 3,6 \\
\hline
\end{tabular}


Table 4-Continued

\begin{tabular}{|c|c|c|c|c|c|c|}
\hline Nova & $\begin{array}{c}\Delta \alpha \cos \delta \\
\quad\left({ }^{\prime}\right)\end{array}$ & $\begin{array}{l}\Delta \delta \\
(')\end{array}$ & $\begin{array}{c}a \\
\left({ }^{\prime}\right)\end{array}$ & $\begin{array}{c}\text { Discovery } \\
\text { mag (Filter) }\end{array}$ & Type & References $^{\mathrm{a}}$ \\
\hline M31N 2007-06b & -2.12 & -15.71 & 26.20 & $16.8(\mathrm{w})$ & $\mathrm{He} / \mathrm{N}$ & 3,7 \\
\hline M31N 2007-07b & 0.29 & 1.92 & 2.26 & $17.7(\mathrm{R})$ & $\mathrm{Fe}$ II & 8 \\
\hline M31N 2007-07c & 3.56 & -1.26 & 5.55 & $15.8(\mathrm{R})$ & $\mathrm{He} / \mathrm{N}$ & 8,9 \\
\hline M31N 2007-07e & -0.20 & 1.59 & 2.00 & $18.1(\mathrm{R})$ & Fe II & 8 \\
\hline M31N 2007-07f & -45.77 & -22.95 & 81.74 & $17.4(\mathrm{w})$ & $\mathrm{Fe} I I$ & 10 \\
\hline M31N 2007-08a & -20.78 & -22.25 & 30.49 & $17.6(\mathrm{w})$ & Fe II: & 8 \\
\hline M31N 2007-08d & -36.91 & -46.74 & 59.56 & 18.1(R) & Fe II & 3 \\
\hline M31N 2007-10a & 2.19 & -12.78 & 27.57 & $16.0(\mathrm{w})$ & $\mathrm{He} / \mathrm{Nn}$ & 3,11 \\
\hline M31N 2007-10b & 8.48 & 1.09 & 12.91 & $17.8(\mathrm{w})$ & $\mathrm{He} / \mathrm{Nn}$ & 12 \\
\hline M31N 2007-11b & 12.94 & -12.52 & 59.72 & $18.6(\mathrm{R})$ & $\mathrm{He} / \mathrm{Nn}$ & $3,13,14$ \\
\hline M31N 2007-11c & 3.72 & -0.24 & 4.77 & $17.4(\mathrm{R})$ & $\mathrm{Fe} I I$ & 14,15 \\
\hline M31N 2007-11d & 24.34 & 21.60 & 34.03 & $14.9(\mathrm{w})$ & Fe II & 3,16 \\
\hline M31N 2007-11e & 34.06 & 46.07 & 57.52 & $16.4(\mathrm{w})$ & $\mathrm{Fe} I \mathrm{II}$ & 3,17 \\
\hline M31N 2007-12a & 14.79 & 22.57 & 28.53 & $17.8(\mathrm{w})$ & Fe II & 3 \\
\hline M31N 2007-12b & 6.70 & -2.37 & 14.79 & $16.1(\mathrm{w})$ & $\mathrm{He} / \mathrm{N}$ & 3,18 \\
\hline M31N 2007-12c & 27.26 & 4.08 & 66.22 & $16.4(\mathrm{w})$ & $\mathrm{Fe}$ II & 19 \\
\hline M31N 2007-12d & -9.30 & -6.35 & 11.92 & $17.2(\mathrm{R})$ & $\mathrm{He} / \mathrm{N}$ & 3 \\
\hline M31N 2008-05c & 5.20 & 3.12 & 6.18 & $17.0(\mathrm{R})$ & $\mathrm{Fe} I I$ & 20 \\
\hline M31N 2008-06b & -3.11 & -1.34 & 3.56 & $15.9(\mathrm{R})$ & $\mathrm{He} / \mathrm{N}$ & 21 \\
\hline M31N 2008-07a & -1.87 & 2.12 & 4.34 & $18.3(\mathrm{R})$ & $\mathrm{Fe}$ II & 22 \\
\hline M31N 2008-07b & 8.08 & -6.08 & 26.01 & $19.0(\mathrm{~g})$ & $\mathrm{Fe} I \mathrm{II}$ & 23 \\
\hline M31N 2008-08a & 0.12 & 0.98 & 1.09 & $16.8(\mathrm{R})$ & $\mathrm{Fe} I \mathrm{II}$ & 24,25 \\
\hline M31N 2008-08b & 1.51 & 0.07 & 1.69 & $16.4(\mathrm{R})$ & $\mathrm{He} / \mathrm{N}$ & 24,25 \\
\hline M31N 2008-08c & -0.72 & 10.15 & 18.72 & $16.8(\mathrm{R})$ & $\mathrm{Fe}$ II & 25 \\
\hline M31N 2008-08d & 33.62 & 106.41 & 109.45 & $18.1(\mathrm{w})$ & Fe II & 26 \\
\hline M31N 2008-09a & -10.85 & -8.26 & 14.26 & $18.1(\mathrm{~g})$ & $\mathrm{Fe} I \mathrm{II}$ & 3 \\
\hline M31N 2008-09c & 1.33 & -14.25 & 30.22 & $17.6(\mathrm{~g})$ & Fe II & 3 \\
\hline M31N 2008-10a & 9.51 & 38.60 & 65.42 & $17.1(\mathrm{w})$ & Fe II & 3 \\
\hline M31N 2008-10b & 3.40 & -1.98 & 6.19 & $18.3(\mathrm{R})$ & Fe II & 25,27 \\
\hline M31N 2008-11a & -13.58 & -10.11 & 18.51 & $16.5(\mathrm{R})$ & $\mathrm{He} / \mathrm{N}$ & 3 \\
\hline M31N 2008-12b & 3.85 & 1.72 & 4.43 & $16.8(\mathrm{w})$ & $\mathrm{Fe} I I$ & 28 \\
\hline M31N 2009-01a & 22.44 & 7.39 & 48.17 & $18.5(\mathrm{w})$ & Fe II & 3 \\
\hline M31N 2009-02a & 11.11 & 20.52 & 26.38 & $16.8(\mathrm{w})$ & $\mathrm{Fe} I \mathrm{II}$ & 3 \\
\hline M31N 2009-08a & 2.57 & 1.35 & 3.00 & $17.2(\mathrm{H})$ & Fe II & 29 \\
\hline M31N 2009-08b & 15.93 & 32.73 & 45.52 & $17.1(\mathrm{w})$ & $\mathrm{Fe} I \mathrm{II}$ & 30 \\
\hline M31N 2009-08d & 0.45 & -0.52 & 0.81 & $17.2(\mathrm{R})$ & Fe II & 31 \\
\hline M31N 2009-08e & -1.54 & 1.89 & 3.54 & $17.8(\mathrm{w})$ & $\mathrm{Fe} I \mathrm{II}$ & 32 \\
\hline M31N 2009-09a & -3.45 & -12.13 & 17.38 & 17.6(w) & $\mathrm{Fe} I \mathrm{II}$ & 33 \\
\hline M31N 2009-10a & 27.78 & 48.61 & 61.67 & $17.1(\mathrm{w})$ & Fe II & 34 \\
\hline M31N 2009-10b & -4.43 & 0.60 & 6.17 & $14.7(\mathrm{R})$ & $\mathrm{Fe}$ II & 35 \\
\hline M31N 2009-10c & 0.26 & -0.19 & 0.37 & $17.2(\mathrm{H})$ & Fe II & 36 \\
\hline M31N 2009-11a & 3.81 & 24.99 & 48.41 & $17.6(\mathrm{R})$ & $\mathrm{Fe} I \mathrm{II}$ & 37 \\
\hline M31N 2009-11b & -0.89 & -7.09 & 10.56 & $18.4(\mathrm{R})$ & Fe II & 23,38 \\
\hline M31N 2009-11c & 4.91 & -3.83 & 12.92 & $17.0(\mathrm{R})$ & $\mathrm{Fe} I \mathrm{II}$ & 39 \\
\hline M31N 2009-11d & 17.37 & 2.79 & 35.48 & $16.4(\mathrm{r})$ & $\mathrm{Fe} I \mathrm{II}$ & 40 \\
\hline
\end{tabular}


Table 4-Continued

\begin{tabular}{cccccrr}
\hline \hline Nova & $\begin{array}{c}\Delta \alpha \cos \delta \\
\left({ }^{\prime}\right)\end{array}$ & $\begin{array}{c}\Delta \delta \\
\left({ }^{\prime}\right)\end{array}$ & $\begin{array}{c}a \\
\left({ }^{\prime}\right)\end{array}$ & $\begin{array}{c}\text { Discovery } \\
\text { mag (Filter) }\end{array}$ & Type & References $^{\mathrm{a}}$ \\
\hline M31N 2009-11e & -1.70 & -3.16 & 3.86 & $17.4(\mathrm{R})$ & Fe II & 41 \\
\hline
\end{tabular}

a REFERENCES: (1) Ciardullo et al. (1983); (2) Tomaney \& Shafter (1992); (3) this work; (4) Pietsch et al. (2005); (5) Hatzidimitriou et al. (2007); (6) Pietsch et al. (2007a); (7) Shafter \& Quimby (2007); (8) Barsukova et al. (2007); (9) Rau et al. (2007a); (10) Quimby (2007, private communication); (11) Gal-Yam \& Quimby (2007); (12) Rau et al. (2007b); (13) Rau (2007); (14) Barsukova et al. (2007b); (15) Ciroi (2007); (16) Shafter et al. (2009); (17) Di Mille et al. (2007); (18) Bode et al. (2009); (19) Rau \& Cenko (2007); (20) Rau et al. (2008) (21) Reig et al. (2008); (22) Barsukova et al. (2008); (23) Kasliwal et al. (2011); (24) Di Mille et al. (2008a); (25) Di Mille et al. (2010); (26) Chornock et al. (2008); (27) Di Mille et al. (2008b); (28) Kasliwal et al. (2008); (29) Valeev et al. (2009); (30) Rodríguez-Gil et al. (2009); (31) Di Mille et al. (2009a); (32) Medvedev et al. (2000); (33) Barsukova et al. (2009a); (34) Fabrika et al. (2009a); (35) Barsukova et al. (2009b); (36) Fabrika et al. (2009b); (37) Hornoch et al. (2009a); (38) Kasliwal (2009); (39) Hornoch et al. (2009b); (40) Hornoch et al. (2009c); (41) Hornoch et al. (2009d)

${ }^{\mathrm{b}}$ The line with of $4400 \mathrm{~km} \mathrm{~s}^{-1}$ reported by D. C. Leonard referred to an estimate of the full width at zero intensity, not the FWHM. Analysis of the original spectrum reveals the object to be a classic Fe II nova 
Table 5. Balmer Emission-Line Properties

\begin{tabular}{ccccc}
\hline \hline & \multicolumn{2}{c}{$\mathrm{EW}$} & $(\mathrm{A})$ & \multicolumn{2}{c}{$\mathrm{FWHM}$} & $\left(\mathrm{km} \mathrm{s}^{-1}\right)$ \\
Nova & $\mathrm{H} \beta$ & $\mathrm{H} \alpha$ & $\mathrm{H} \beta$ & $\mathrm{H} \alpha$ \\
\hline M31N 1990-10b & -267 & -541 & 1600 & 1550 \\
M31N 1992-11b & -250 & -1219 & 1670 & 1740 \\
M31N 1993-06a & -36 & -392 & 1840 & 1770 \\
M31N 1993-08a & $\ldots$ & $\ldots$ & $\ldots$ & 4350 \\
M31N 1993-10g & -87 & -324 & 1630 & 1680 \\
M31N 1993-11c & -118 & -828 & 1450 & 1560 \\
M31N 1998-09d & -92 & -323 & 1720 & 1840 \\
M31N 1999-06a & -322 & -1200 & 1110 & 920 \\
M31N 1999-08f & -135 & -704 & 1640 & 1690 \\
M31N 1999-10a & -230 & -565 & 1780 & 1930 \\
M31N 2001-10a & -279 & -945 & 1540 & 1540 \\
M31N 2001-12a & $\ldots$ & -1500 & $\ldots$ & 1310 \\
M31N 2002-01b & $\ldots$ & -760 & $\ldots$ & 3430 \\
M31N 2002-08a & -94 & -470 & 1360 & 1320 \\
M31N 2004-08b & -90 & -410 & 1970 & 1830 \\
M31N 2004-09a & -54 & -250 & 2000 & 1880 \\
M31N 2004-11a & -175 & -830 & 1230 & 1580 \\
M31N 2004-11b & -80 & -1020 & 2500 & 2620 \\
M31N 2005-01a & -50 & -107 & 1810 & 1680 \\
M31N 2005-07a & $\ldots$ & -120 & $\ldots$ & 1100 \\
M31N 2006-09c & -127 & -470 & 1910 & 1920 \\
M31N 2006-10a & -44 & -90 & 950 & 810 \\
M31N 2006-10b & -64 & -102 & 3330 & 3090 \\
M31N 2008-09a & -113 & -262 & 1540 & 1460 \\
M31N 2006-11a & -335 & -2130 & 3030 & 3562 \\
M31N 2006-12a & -107 & -330 & 1850 & 1760 \\
M31N 2006-12b & -86 & -226 & 1230 & 1020 \\
M31N 2007-02a & -35 & -61 & 1530 & 1310 \\
M31N 2007-02b & -39 & -304 & 1460 & 1910 \\
M31N 2007-06b & -51 & -168 & 2940 & 2870 \\
M31N 2007-08d & -68 & -284 & 1160 & 1180 \\
M31N 2007-10a & -37 & -154 & 470 & 500 \\
M31N 2007-11b & -147 & -535 & 1480 & 1290 \\
M31N 2007-11c & -75 & -137 & 1950 & 1700 \\
M31N 2007-11d & -11 & -16 & 1630 & 1550 \\
M31N 2007-11e & -137 & -306 & 1750 & 1600 \\
M31N 2007-11g & -18 & -13 & 340 & 300 \\
M31N 2007-12a & -159 & -309 & 2050 & 1850 \\
M31N 2007-12b & -247 & -990 & 4070 & 4080 \\
M31N 2008-12d & -271 & -1210 & 5220 & 4980 \\
& -130 & -440 & 1150 & 1050 \\
M30 & -23 & 1590 & 1010 \\
M30 & & & &
\end{tabular}


Table 5-Continued

\begin{tabular}{ccccc}
\hline \hline & \multicolumn{2}{c}{$\mathrm{EW}(\AA)$} & \multicolumn{2}{c}{$\mathrm{FWHM}\left(\mathrm{km} \mathrm{s}^{-1}\right)$} \\
Nova & $\mathrm{H} \beta$ & $\mathrm{H} \alpha$ & $\mathrm{H} \beta$ & $\mathrm{H} \alpha$ \\
\hline M31N 2008-10a & -97 & -300 & 1390 & 1270 \\
M31N 2008-10b & -2.8 & -19 & 660 & 640 \\
& -24 & -95 & 1220 & 950 \\
M31N 2008-11a & -188 & -636 & 4510 & 4350 \\
& -33 & -370 & 1460 & 3160 \\
M31N 2009-01a & -5 & -25 & 560 & 550 \\
M31N 2009-02a & -4 & -15 & 1280 & 1410 \\
\hline
\end{tabular}

${ }^{\text {a }}$ Due to ambiguity in the data logs from November 1993, it is possible that the data for these two novae are reversed. 
Table 6. Light-Curve Parameters

\begin{tabular}{|c|c|c|c|c|}
\hline Nova & Filter & $M_{\max }$ & Fade Rate (mag day ${ }^{-1}$ ) & $t_{2}$ (days) \\
\hline M31N 1999-08f & $r^{\prime}$ & $-7.54 \pm 0.11$ & $0.063 \pm 0.002$ & $31.7 \pm 0.9$ \\
\hline M31N 2001-10a & $r^{\prime}$ & $-7.54 \pm 0.11$ & $0.040 \pm 0.004$ & $50.2 \pm 4.9$ \\
\hline M31N 2002-08a & $R$ & $-7.49 \pm 0.20$ & $0.044 \pm 0.005$ & $45.8 \pm 5.1$ \\
\hline M31N 2004-08b & $R$ & $-7.24 \pm 0.25$ & $0.038 \pm 0.002$ & $52.0 \pm 3.3$ \\
\hline M31N 2004-09a & $R$ & $-7.10 \pm 0.20$ & $0.056 \pm 0.006$ & $35.5 \pm 3.7$ \\
\hline M31N 2004-11a & $R$ & $-8.04 \pm 0.25$ & $0.105 \pm 0.010$ & $19.1 \pm 1.9$ \\
\hline M31N 2004-11b & $R$ & $-7.94 \pm 0.20$ & $0.042 \pm 0.002$ & $47.2 \pm 2.6$ \\
\hline M31N 2005-01a & $R$ & $-9.59 \pm 0.17$ & $0.105 \pm 0.009$ & $19.0 \pm 1.6$ \\
\hline M31N 2005-07a & $R$ & $-7.16 \pm 0.25$ & $0.017 \pm 0.003$ & $120.9 \pm 23.5$ \\
\hline M31N 2006-06a & $R$ & $-7.04 \pm 0.11$ & $0.030 \pm 0.006$ & $67.3 \pm 13.2$ \\
\hline M31N 2006-09c & $R$ & $-7.88 \pm 0.14$ & $0.087 \pm 0.006$ & $23.1 \pm 1.6$ \\
\hline M31N 2006-10a & $B$ & $-7.54 \pm 0.11$ & $0.035 \pm 0.002$ & $56.7 \pm 3.1$ \\
\hline$\ldots$ & $V$ & $-7.67 \pm 0.11$ & $0.035 \pm 0.002$ & $57.5 \pm 3.1$ \\
\hline$\ldots$ & $R$ & $-6.64 \pm 0.20$ & $0.021 \pm 0.005$ & $94.3 \pm 20.6$ \\
\hline M31N 2006-10b & $B$ & $-7.85 \pm 0.11$ & $0.287 \pm 0.018$ & $7.0 \pm 0.4$ \\
\hline$\ldots$ & $V$ & $-7.98 \pm 0.11$ & $0.345 \pm 0.018$ & $5.8 \pm 0.3$ \\
\hline M31N 2006-11a & $R$ & $-8.54 \pm 0.20$ & $0.070 \pm 0.006$ & $28.7 \pm 2.6$ \\
\hline M31N 2006-12a & $R$ & $-7.24 \pm 0.25$ & $0.057 \pm 0.007$ & $34.9 \pm 4.5$ \\
\hline M31N 2007-02b & $R$ & $-7.88 \pm 0.20$ & $0.059 \pm 0.006$ & $34.1 \pm 3.6$ \\
\hline M31N 2007-07e & $R$ & $-6.64 \pm 0.35$ & $0.044 \pm 0.010$ & $45.6 \pm 10.5$ \\
\hline M31N 2007-08d & $R$ & $-6.44 \pm 0.11$ & $0.025 \pm 0.003$ & $80.5 \pm 10.5$ \\
\hline M31N 2007-10a & $B$ & $-8.25 \pm 0.11$ & $0.222 \pm 0.013$ & $9.0 \pm 0.5$ \\
\hline$\ldots$ & $V$ & $-8.38 \pm 0.11$ & $0.255 \pm 0.013$ & $7.9 \pm 0.4$ \\
\hline$\ldots$ & $i^{\prime}$ & $-6.51 \pm 0.13$ & $0.225 \pm 0.010$ & $8.9 \pm 0.4$ \\
\hline M31N 2007-10b & $B$ & $-7.60 \pm 1.15$ & $0.508 \pm 0.067$ & $3.9 \pm 0.5$ \\
\hline$\ldots$ & $V$ & $-8.14 \pm 1.56$ & $0.690 \pm 0.065$ & $2.9 \pm 0.3$ \\
\hline$\ldots$ & $R$ & $-8.18 \pm 1.44$ & $0.639 \pm 0.085$ & $3.1 \pm 0.4$ \\
\hline M31N 2007-11b & $B$ & $-5.65 \pm 0.11$ & $0.055 \pm 0.004$ & $36.3 \pm 2.6$ \\
\hline$\ldots$ & $V$ & $-5.78 \pm 0.11$ & $0.044 \pm 0.003$ & $45.4 \pm 3.5$ \\
\hline$\ldots$ & $R$ & $-6.36 \pm 0.25$ & $0.046 \pm 0.012$ & $43.6 \pm 11.0$ \\
\hline$\ldots$ & $i^{\prime}$ & $-5.45 \pm 0.14$ & $0.027 \pm 0.006$ & $74.4 \pm 16.7$ \\
\hline M31N 2007-11c & $B$ & $-8.25 \pm 0.24$ & $0.138 \pm 0.006$ & $14.5 \pm 0.6$ \\
\hline$\ldots$ & $V$ & $-8.20 \pm 0.27$ & $0.155 \pm 0.007$ & $12.9 \pm 0.6$ \\
\hline$\ldots$ & $i^{\prime}$ & $-7.33 \pm 0.18$ & $0.171 \pm 0.013$ & $11.7 \pm 0.9$ \\
\hline M31N 2007-11d & $B$ & $-9.35 \pm 0.11$ & $0.143 \pm 0.009$ & $14.0 \pm 0.9$ \\
\hline$\ldots$ & $V$ & $-9.48 \pm 0.11$ & $0.158 \pm 0.009$ & $12.6 \pm 0.7$ \\
\hline$\ldots$ & $r^{\prime}$ & $-9.64 \pm 0.11$ & $0.153 \pm 0.009$ & $13.1 \pm 0.8$ \\
\hline$\ldots$ & $i^{\prime}$ & $-8.72 \pm 0.11$ & $0.216 \pm 0.011$ & $9.2 \pm 0.5$ \\
\hline M31N 2007-12a & $B$ & $-7.02 \pm 0.12$ & $0.078 \pm 0.005$ & $25.6 \pm 1.6$ \\
\hline$\ldots$ & $V$ & $-7.06 \pm 0.11$ & $0.081 \pm 0.004$ & $24.8 \pm 1.4$ \\
\hline$\ldots$ & $i^{\prime}$ & $-7.19 \pm 0.11$ & $0.068 \pm 0.004$ & $29.6 \pm 2.0$ \\
\hline M31N 2007-12b & $B$ & $-8.15 \pm 0.11$ & $0.497 \pm 0.034$ & $4.0 \pm 0.3$ \\
\hline$\ldots$ & $V$ & $-8.28 \pm 0.11$ & $0.548 \pm 0.033$ & $3.6 \pm 0.2$ \\
\hline$\ldots$ & $R$ & $-8.44 \pm 0.11$ & $0.403 \pm 0.043$ & $5.0 \pm 0.5$ \\
\hline M31N 2008-05c & $R$ & $-7.54 \pm 0.11$ & $0.038 \pm 0.002$ & $53.0 \pm 2.8$ \\
\hline M31N 2008-06b & $R$ & $-8.64 \pm 0.11$ & $0.083 \pm 0.005$ & $24.2 \pm 1.5$ \\
\hline
\end{tabular}


Table 6-Continued

\begin{tabular}{|c|c|c|c|c|}
\hline Nova & Filter & $M_{\max }$ & Fade Rate $\left(\operatorname{mag}\right.$ day $\left.^{-1}\right)$ & $t_{2}$ (days) \\
\hline M31N 2008-07a & $R$ & $-6.24 \pm 0.11$ & $0.005 \pm 0.001$ & $410.0 \pm 51.3$ \\
\hline M31N 2008-07b & $R$ & $-6.14 \pm 0.20$ & $0.049 \pm 0.006$ & $40.9 \pm 4.7$ \\
\hline M31N 2008-08a & $R$ & $-8.04 \pm 0.20$ & $0.094 \pm 0.011$ & $21.2 \pm 2.5$ \\
\hline M31N 2008-10a & $B$ & $-7.15 \pm 0.11$ & $0.053 \pm 0.003$ & $37.7 \pm 2.2$ \\
\hline$\ldots$ & $V$ & $-7.28 \pm 0.11$ & $0.052 \pm 0.003$ & $38.3 \pm 2.2$ \\
\hline$\ldots$ & $r^{\prime}$ & $-7.44 \pm 0.11$ & $0.030 \pm 0.003$ & $67.5 \pm 6.7$ \\
\hline M31N 2008-10b & $B$ & $-7.08 \pm 0.12$ & $0.020 \pm 0.003$ & $98.4 \pm 14.9$ \\
\hline$\ldots$ & $V$ & $-6.88 \pm 0.12$ & $0.017 \pm 0.003$ & $117.3 \pm 21.5$ \\
\hline$\ldots$ & $R$ & $-6.56 \pm 0.25$ & $0.032 \pm 0.015$ & $63.3 \pm 30.2$ \\
\hline$\ldots$ & $r^{\prime}$ & $-6.71 \pm 0.11$ & $0.027 \pm 0.003$ & $73.2 \pm 8.1$ \\
\hline M31N 2008-11a & $B$ & $-7.75 \pm 0.11$ & $0.522 \pm 0.029$ & $3.8 \pm 0.2$ \\
\hline$\ldots$ & $V$ & $-7.88 \pm 0.11$ & $0.457 \pm 0.024$ & $4.4 \pm 0.2$ \\
\hline$\ldots$ & $R$ & $-8.04 \pm 0.11$ & $0.378 \pm 0.044$ & $5.3 \pm 0.6$ \\
\hline$\ldots$ & $r^{\prime}$ & $-8.04 \pm 0.11$ & $0.253 \pm 0.013$ & $7.9 \pm 0.4$ \\
\hline$\ldots$ & $i^{\prime}$ & $-6.13 \pm 0.13$ & $0.120 \pm 0.006$ & $16.6 \pm 0.8$ \\
\hline$\ldots$ & $z^{\prime}$ & $-6.61 \pm 0.12$ & $0.237 \pm 0.017$ & $8.4 \pm 0.6$ \\
\hline M31N 2008-12b & $B$ & $-7.48 \pm 0.11$ & $0.055 \pm 0.006$ & $36.7 \pm 4.1$ \\
\hline$\ldots$ & $V$ & $-7.61 \pm 0.11$ & $0.065 \pm 0.006$ & $30.7 \pm 2.9$ \\
\hline$\ldots$ & $r^{\prime}$ & $-7.74 \pm 0.11$ & $0.073 \pm 0.006$ & $27.6 \pm 2.2$ \\
\hline$\ldots$ & $i^{\prime}$ & $-7.00 \pm 0.15$ & $0.081 \pm 0.012$ & $24.7 \pm 3.6$ \\
\hline$\ldots$ & $z^{\prime}$ & $-7.73 \pm 0.12$ & $0.044 \pm 0.011$ & $45.0 \pm 10.9$ \\
\hline M31N 2009-08a & $B$ & $-7.05 \pm 0.11$ & $0.006 \pm 0.001$ & $351.1 \pm 84.5$ \\
\hline$\ldots$ & $V$ & $-7.18 \pm 0.11$ & $0.011 \pm 0.001$ & $190.0 \pm 24.5$ \\
\hline$\ldots$ & $R$ & $-7.34 \pm 0.11$ & $0.014 \pm 0.001$ & $142.3 \pm 9.0$ \\
\hline$\ldots$ & $r^{\prime}$ & $-7.34 \pm 0.11$ & $0.014 \pm 0.001$ & $142.3 \pm 13.1$ \\
\hline M31N 2009-08b & $B$ & $-7.15 \pm 0.11$ & $0.111 \pm 0.005$ & $18.0 \pm 0.8$ \\
\hline$\ldots$ & $V$ & $-7.28 \pm 0.11$ & $0.112 \pm 0.005$ & $17.8 \pm 0.8$ \\
\hline$\ldots$ & $r^{\prime}$ & $-7.44 \pm 0.11$ & $0.086 \pm 0.004$ & $23.1 \pm 1.2$ \\
\hline$\ldots$ & $i^{\prime}$ & $-6.47 \pm 0.12$ & $0.074 \pm 0.006$ & $26.9 \pm 2.2$ \\
\hline M31N 2009-08d & $B$ & $-7.23 \pm 0.24$ & $0.072 \pm 0.017$ & $27.9 \pm 6.5$ \\
\hline$\ldots$ & $V$ & $-7.18 \pm 0.11$ & $0.063 \pm 0.008$ & $31.7 \pm 3.9$ \\
\hline$\ldots$ & $R$ & $-7.34 \pm 0.20$ & $0.055 \pm 0.008$ & $36.2 \pm 5.2$ \\
\hline$\ldots$ & $r^{\prime}$ & $-7.34 \pm 0.11$ & $0.077 \pm 0.019$ & $25.9 \pm 6.5$ \\
\hline M31N 2009-08e & $R$ & $-6.74 \pm 0.11$ & $0.016 \pm 0.001$ & $121.3 \pm 8.0$ \\
\hline M31N 2009-09a & $R$ & $-7.04 \pm 0.25$ & $0.012 \pm 0.001$ & $163.7 \pm 15.4$ \\
\hline M31N 2009-10a & $B$ & $-7.15 \pm 0.11$ & $0.123 \pm 0.007$ & $16.2 \pm 0.9$ \\
\hline$\ldots$ & $V$ & $-7.28 \pm 0.11$ & $0.132 \pm 0.006$ & $15.2 \pm 0.7$ \\
\hline M31N 2009-10b & $B$ & $-9.55 \pm 0.11$ & $0.250 \pm 0.007$ & $8.0 \pm 0.2$ \\
\hline$\ldots$ & $V$ & $-9.68 \pm 0.11$ & $0.224 \pm 0.005$ & $8.9 \pm 0.2$ \\
\hline$\ldots$ & $R$ & $-9.84 \pm 0.11$ & $0.156 \pm 0.010$ & $12.8 \pm 0.8$ \\
\hline M31N 2009-10c & $B$ & $-8.75 \pm 0.13$ & $0.134 \pm 0.008$ & $14.9 \pm 0.8$ \\
\hline$\ldots$ & $V$ & $-8.24 \pm 0.14$ & $0.124 \pm 0.011$ & $16.1 \pm 1.4$ \\
\hline$\ldots$ & $R$ & $-8.14 \pm 0.25$ & $0.065 \pm 0.020$ & $30.6 \pm 9.4$ \\
\hline M31N 2009-11a & $B$ & $-6.65 \pm 0.11$ & $0.095 \pm 0.007$ & $21.1 \pm 1.6$ \\
\hline$\ldots$ & $V$ & $-6.78 \pm 0.11$ & $0.092 \pm 0.005$ & $21.7 \pm 1.2$ \\
\hline$\ldots$ & $R$ & $-6.94 \pm 0.11$ & $0.096 \pm 0.009$ & $20.8 \pm 1.9$ \\
\hline
\end{tabular}


Table 6-Continued

\begin{tabular}{lcccc}
\hline \hline \multicolumn{1}{c}{ Nova } & Filter & $M_{\max }$ & Fade Rate $\left(\mathrm{mag}_{\text {day }}{ }^{-1}\right)$ & $t_{2}($ days $)$ \\
\hline M31N 2009-11b & $B$ & $-5.85 \pm 0.11$ & $0.022 \pm 0.004$ & $92.5 \pm 16.6$ \\
$\ldots$ & $V$ & $-5.98 \pm 0.11$ & $0.027 \pm 0.004$ & $74.8 \pm 10.6$ \\
$\ldots$ & $R$ & $-6.14 \pm 0.11$ & $0.023 \pm 0.002$ & $88.0 \pm 6.6$ \\
M31N 2009-11c & $B$ & $-7.28 \pm 0.11$ & $0.048 \pm 0.003$ & $42.0 \pm 2.7$ \\
$\ldots$ & $V$ & $-7.84 \pm 0.14$ & $0.062 \pm 0.005$ & $32.5 \pm 2.4$ \\
$\ldots$ & $R$ & $-7.57 \pm 0.11$ & $0.046 \pm 0.004$ & $43.1 \pm 3.6$ \\
M31N 2009-11d & $B$ & $-7.85 \pm 0.11$ & $0.178 \pm 0.007$ & $11.2 \pm 0.4$ \\
$\ldots$ & $V$ & $-7.98 \pm 0.11$ & $0.276 \pm 0.025$ & $7.2 \pm 0.7$ \\
$\ldots$ & $R$ & $-8.14 \pm 0.11$ & $0.122 \pm 0.013$ & $16.3 \pm 1.7$ \\
M31N 2009-11e & $R$ & $-7.56 \pm 0.20$ & $0.036 \pm 0.002$ & $55.7 \pm 3.1$ \\
\hline
\end{tabular}

